Historic, archived document

Do not assume content reflects current scientific knowledge, policies, or practices. 



\section{The Potential for Genetic Suppression of Insect Populations by Their Adaptations to Climate}

Miscellaneous Publication No. 1178 


\section{Acknowledgments}

The use of the IBM System/360 computer was made possible through the cooperative agreement between North Dakota State University, Fargo, and the Metabolism and Radiation Research Laboratory, Agricultural Research Service.

We are grateful to E. F. Knipling and D. A. Wolfenbarger of the Entomology Research Division, Agricultural Research Service, and to G. W. K. Stehr, Department of Fisheries and Forestry, Sault Sainte Marie, Ontario, Canada, for their constructive criticisms and helpful suggestions during this study.

\section{Contents}

Native population overflooded with release strain bearing conditional lethal traits

Trade names are used in this publication solely to provide specific information. Mention of a trade name does not constitute a guarantee or warranty of the product by the U.S. Department of Agriculture or an endorsement by the Department over other products not mentioned. 


\title{
The Potential for Genetic Suppression of Insect Populations by Their Adaptations to Climate
}

\author{
By W. Klassex, J. F. CReech, and R. A. Bell ${ }^{1}$
}

Many of our most serious insect pests have rery broad geographical distributions. For example, the codling moth (Carpocapsa pomonella (L.)) is distributed from Canada to Argentina, and it occurs in Australia, South Africa, the Mediterranean, and throughout Europe north to the Scandinavian countries (1).2 Insects with such broad distributions adapt in various ways to climate.

Many of the reports $(2,3,5,6)$ indicate that genetic differences occur between insect populations within a species with regard to (1) ability to undergo a hibernal diapause, (2) response to diapause-inducing stimuli, (3) duration of diapause, (4) temperature limits of diapause termination, (5) temperature optima for diapause termination, (6) ability to develop cold hardiness, ( 7 ) thermal constants and temperature threshold for development, (8) choice of hibernal niches and other behavioral traits associated with surviving inhospitable seasons, and (9) ability for aestival diapause, its duration and response to conditions that induce or terminate it.

Such genetic differences must exist so that adaptations of insects to climate may be appropriate to their locality. Changes in climate from locality to locality require appropriate changes in adaptations. Insects must synchronize their life cycles with the seasons so that (1) frostsensitive stages are passed in the frost-free sea-

${ }^{1}$ W. Klassen and R. A. Bell, entomologists, Entomology Research Division, Agricultural Research Service, and J. F. Creech, graduate student, Department of Electrical Engineering, North Dakota State University.

${ }^{2}$ Italic numbers in parentheses refer to Literature Cited, p. 10. son, (2) feeding stages occur when food is available, and (3) no actively developing stages occul in periods of intense heat or drought. Therefore insects must be sensitive to stimuli that portend the change in seasons so that they may prepare for adverse periods.

If we can genetically disrupt the seasonal regulations or other climatic adaptations of insects, these insects may not survive. For example, if an insect population at Fargo, N. Dak., must respond to a photoperiod of 15 hours in order to enter diapause and be cold hardy in time for dangerous frosts and if we genetically modify the population so that it does not diapause until the day has shortened to 13 hours, then the population may be destroyed by the winter. Further, let us assume that this population must remain in diapause until early May, when the danger of killing frost is past and when the host plant has again become available. If we genetically shorten the diapause so that the insects resume development in March, then they would be destroyed by frost or lack of food.

Inappropriate adaptations to climate are lethal at certain times of the year; they are conditional lethal traits. We may use a conditional lethal trait or combination of conditional lethal traits to suppress or eradicate insect populations. The principle of suppressing insect populations by means of their adaptations to climate has been suggested by numerous investigators $(4,5,7)$.

The purpose of this report is to show that a population may be suppressed by means of conditional lethal traits, particularly (1) when the population is held in check by partial sterility while conditional lethal genes are introduced and (2) when the population is held static by conventional means while the conditional lethal 
genes are introduced. Each conditional lethal trait may be determined by one to four genes, and two or three conditional lethal traits may be simultaneously introduced into the population.

Conditional lethal traits may be found in the genetic variations in adaptations to climate and to seasonal regulations. A few examples are (1) inability to diapause, (2) inappropriate duration of diapause, (3) inappropriate critical thresholds of diapause-inducing stimuli such as photoperiod, temperature, or dietary factors, (4) inappropriate choice of hibernal niches, and (5) inability to develop cold hardiness.

\section{Native Population Overflooded}

\section{With Release Strain Bearing Conditional Lethal Traits}

A preliminary evaluation of the potential for insect population suppression by conditional lethal traits was made by Klassen and others (5). They considered the suppressive effect of a trait determined by one, two, or three autosomal genes and by three autosomal genes plus one sex-linked gene. In addition, these authors calculated the suppressive effect when two or three conditional lethal traits, each determined by a single dominant gene, are combined in the release strain. We decided to expand these calculations and to extend them to release strains with two or three conditional lethal traits. Each trait was determined by one, two, three, or four independent genes as follows:

One Dominant Autosomal Gene.-The genotypes that were inviable when the conditional lethal trait was expressed were $\mathrm{AA}$ and $\mathrm{Aa}$, whereas aa was the only viable genotype.

Two Autosomal Genes With Additive Effects $(a a b b)$.- - In the examples computed, each of the four alleles was assigned a genotypic value of 1 or 0 . The native strain AABB had a genotypic value of 4 , and the release strain aabb had a genotypic value of 0 . We assumed that the conditional lethal trait was expressed in individuals with genotypic values of 0,1 , and 2 . Therefore $\mathrm{AABB}, \mathrm{AABb}$, and $\mathrm{AaBB}$ were the only viable genotypes.

Three Autosomal Genes With Additive Effects $(a a b b d d)$. - Each of the six alleles was assigned a genotypic value of 1 or 0 . The native strain $A A B B D D$ had a genotypic value of 6 , and the release strain aabbdd had a genotypic value of 0 . We assumed that the conditional lethal trait was expressed in individuals with genotypic values of $0,1,2$, and 3 . Examples of viable genotypes were $A A B B D D, A a B B D D$, and AABBdd.
Four Autosomal Genes With Additive Effects (aabbddee).-Each of the eight alleles was assigned a genotypic value of 1 or 0 . The native strain AABBDDEE had a genotypic value of 8 , and the release strain aabbddee had a genotypic value of 0 . We assumed that the conditional lethal trait was expressed in individuals with genotypic values of $0,1,2,3,4$, and 5 . Examples of viable genotypes were AABBDDEE, AABBDDee, and AaBbDDEE.

One Dominant Sex-Linked Gene and Three Autosomal Genes With Additive Effects (abbddee).-The sex-linked allele a was assigned a value of 0 and its allele $A$ was assigned a genotypic value of 3 . Since A was fully dominant, the gene AA had a value of 3 . The autosomal factors $\mathrm{B}, \mathrm{D}$, and $\mathrm{E}$ were each assigned a value of 1 , and their respective alleles $b, d$, and e were each assigned a value of 0 . We assumed that the conditional lethal trait was expressed in individuals with genotypic values of $0,1,2$, 3,4 , and 5. Examples of viable genotypes were A-BBDDEE, AABBDDEE, and a-BBDDEE. The heterogametic sex (A-BBDDEE) was assumed to be female, and the homogametic sex (AABBDDEE) was assumed to be male.

Algebraic expressions describing the segregation of genes that determine conditional lethal traits were derived by Klassen and others (5). These authors arbitrarily assigned values or "weights" to each allele so that each genotype could be given a "genotypic value." In addition, they arbitrarily set minimum genotypic values required for survival and gave algebraic expressions to calculate the numbers of surviving genotypes in the $F_{1}, F_{2}$, and $F_{3}$ generations when a native population is overflooded once with a release strain homozygous for the genes 
that determine the conditional lethal traits. The algebraic expressions (table 1. Appendix) enabled us to calculate the fraction $\left(w^{\circ}\right)$ of the affected population that was destroyed by one or more lethal traits and to calculate the suppression of the affected population relative to a native population not subjected to population control measures.

If a strain homozygous for more than one conditional lethal gene is released into a population of native insects, these genes will become distributed throughout the population in an almost random mannel by the $F_{3}$ generation, barring linkage intensities of less than 50 crossover units. Therefore we may approximately calculate the fraction of the population that would be suppressed following a single release of a strain bearing several conditional lethal traits. If the frequency of individuals without lethal trait 1 is $A$ and the frequency without lethal trait 2 is $B$, then the frequency of individuals without either lethal trait is AB. Therefore the frequency of individuals with one or both lethal traits is $1-\mathrm{AB}$, or the fraction of the population suppressed.

The suppression of the population must be calculated in relation to the uncontrolled native population. Let there be $y$ native insects that increase 0 -fold each generation. Then the numbers of insects in the parental, $F_{1}, F_{2}$, and $F_{n}$ generations are

$$
y, y \emptyset, y \emptyset^{2} \text {, and } y \emptyset^{\mathrm{n}}
$$

Similarly if $x$ insects with conditional lethal traits are released into a population of $y$ native insects, then the numbers of insects in the parental, $F_{1}, F_{2}$, and $F_{n}$ generations are

$$
x+y,(x+y) \emptyset,(x+y) \emptyset^{2} \text {, and }(x+y) \emptyset^{n}
$$

In the $n$th generation the conditional lethal traits are expressed in a fraction $(w)$ of the affected population. The number of survivors is

$$
(1-w)(x+y) \emptyset^{n}
$$

Then the relative survival is

$$
(1-w)(x+y) \div y
$$

The release ratio $X\left(X=\frac{x}{y}\right)$ required to obtain 98- to 99.9-percent suppression in the $F_{1}$ and $F_{3}$ generations was calculated (table 2) for conditional lethal traits determined by one dominant (dom.) gene, two autosomal (auto.) genes with additive effects, three autosomal genes with additive effects, and three autosomal genes with additive effects plus one dominant sex-linked (s.l.) gene.

The numbers in parentheses in table 2 are the genotypic values of the survivors. For example, 2 auto. $(3,4)$ indicates the trait is determined by two independent autosomal genes with equal and additive effects and the conditional lethal trait will not be expressed in individuals possessing three or four native-type alleles. Similarly 3 auto. +1 s.l. (6-9) indicates that the trait is determined by three independent autosomal genes with equal and additive effects plus one sex-linked dominant gene with an effect equal to three dominant autosomal alleles. The survivors possess the native-type sex-linked allele and at least three native-type autosomal alleles, or they may lack the native-type sexlinked allele but possess all six native-type autosomal alleles. These calculations were made with an IBM System/360, Model G50 computer, as explained in the next section.

If the release strain is homozygous for only one conditional lethal trait that expresses itself in the $F_{1}$ generation, the degree of suppression obtained cannot exceed that obtained with ordinary dominant lethal mutations. However, if the genes for conditional lethal traits become randomly distributed within the population before they are expressed, the degree of suppression may increase significantly if the trait is under polyfactorial control (table 2). If the conditional lethal trait is determined by a single dominant gene, the degree of suppression cannot vary between generations.

When two conditional lethal traits are combined into a single population, release ratios varying from two to five are adequate to obtain 98-percent suppression if the traits are expressed in the $\mathrm{F}_{3}$ generation; similarly release ratios of three to 14 will yield 99.9-percent suppression in the $F_{3}$ generation (table 2). When three conditional lethal traits are combined into a single population, release ratios of one or two will yield 98-percent suppression in the $\mathrm{F}_{3}$ generation, and ratios of two to five will yield 99.9-percent suppression. Although these values are approximations, they were sufficiently encouraging to undertake the more precise calculations presented in the following sections. 


\section{Suppression of Population Held in Check by Partial Sterility With Conditional Lethal Traits}

Calculations are presented to show the potential for population suppression by conditional lethal genes that are introduced while the population is held in check by partial sterility. Such a method might be more economical and more desirable from the standpoint of avoiding side effects to nontarget organs than that of keeping insects in check with insecticides or other control measures.

For the calculations, we assume that the level of sterility required could be induced without adversely affecting mating competitiveness, sperm competitiveness, or lifespan. The level of sterility required to hold a population constant depends on the rate of increase between generations. For example, if a population normally increased fivefold per generation, then 80-percent sterility in all individuals would prevent a change in numbers. Similarly if a population increased tenfold, then 90-percent sterility of all individuals would prevent a change. Moreover, if a population normally increased fivefold per generation, the 90-percent sterility of all individuals would cause a downward trend in numbers.

To keep a fully fertile native population in check by overflooding with partially sterile individuals, slightly higher levels of sterility are required in the release strain than when all individuals of the native population are partially sterile. The smaller the release ratio, the higher the level of sterility required. Whether matings between partially sterile males and partially sterile females yield offspring has an important bearing on population trends in the calculations presented here.

In our calculations the number of individuals released in each generation remains constant, either 198,000 or 396,000 ; parental generation of the native population consists of 2,000 individuals. Thus the initial ratio of released to native insects is $99: 1$ or $198: 1$. However, if the target population increases, the release ratio diminishes, and the population's growth accelerates after the $F_{1}$ generation and vice versa.

If matings between partially sterile males and partially sterile females produce no offspring, the overall population is held nearly static by the following two sets of conditions: Initial release ratio of $99: 1$ and $198: 1$, each with 90 percent sterility and a fivefold increase between generations. However, when matings between partially sterile males and partially sterile females produce offspring commensurate with the level of sterility, the population increases even when the rate of increase is only fivefold and the initial release ratio is 198:1.

Because of the complexity of the equations involved in arriving at the relative frequency distributions of genotypes and numbers of offspring, especially with three and four genes, we used a digital computer programed to generate from the $F_{1}$ genotypic identification and distribution of offspring, the $F_{2}, F_{3}$, and $F_{4}$ genotypic identifications, offspring distributions, and relative frequencies with provisions to weight the computations as dictated by the particular study performed. We programed in PL/1-F and FORTRAN IV-G and ran under release $15 / 16$ of OS on an IBM System/360, Model 50G (128K), using three IBM 2311 direct-access storage devices for intermediate storage and an IBM 1403500 line-per-minute printer for final output. The computer facility is located in the University Computer Center, North Dakota State University.

The method employed in arriving at the final answers on the computer is similar to and is derived from a method that might be employed if one were to compute the answers by hand (see Appendix, p. 10) If we have a native insect population consisting of $y$ individuals with a genotypic identification of $d d$, and if we release a controlled population consisting of $x$ individuals with a genotypic identification of $D D$, we might calculate the $F_{1}$ generation in the following way:

Let $M$ represent male and $F$ female members.

$$
\begin{aligned}
& d d(M) \times d d(F) \\
& =y^{2} /(x+y) \quad d d \text { males and females } \\
& D D(M) \times D D(F) \\
& =x^{2} /(x+y) \quad D D \text { males and females } \\
& D D(M) \times d d(F) \\
& =x y /(x+y) \quad D d \text { males and females } \\
& d d(M) \times D D(F) \\
& =x y /(x+y) \quad D d \text { males and females }
\end{aligned}
$$


Total, where $z$ is an arbitrary weighting factor:

$$
\begin{array}{lr}
z y^{2}(x+y) & d d \text { males and females } \\
z x^{2}(x-y) & D D \text { males and females } \\
2 z x y(x-y) & D d \text { males and females }
\end{array}
$$

Similarly we might calculate the $\mathrm{F}_{2}, \mathrm{~F}_{3}$, and $F_{n}$ generatious by determining what fraction of each genotype from the previous generation contributes to the current generation when all combinations of the previous generation have been determined. The following method describes our work with four genes and is generally applicable. All programs written for this study had this same form.

All possible genotypes will appear in the $F_{2}$ generation. Therefore the contribution by each $\mathrm{F}_{2}$ mating to a particular $\mathrm{F}_{3}$ genotype will be the same as the contribution from the same $\mathrm{F}_{3}$ mating to the same respective $F_{4}$ genotype. This holds true for all higher generations assuming that no new genotypes are added and none of the existing ones are removed. We have provided a simplified block diagram of the general program flow and the techniques employed in developing computer programs suitable for this study (fig. 1, Appendix).

To make a computer program of this type feasible, we first developed a unique numerical representation for each genotype. Then we developed a method to compute and store the fractional contributions of all combinations of $F_{1}$ genotypes (i.e., matings) to each particular member of the $F_{2}$ generation and the fractional contributions of all combinations of $\mathrm{F}_{2}$ genotypes to each particular $F_{3}$ genotype. The contributions of the latter remain constant for all higher order cases. Therefore upon completion of the $\mathrm{F}_{3}$ generation "formulas," the numerical computations necessary to determine the final answers can be made. We gave each allele a unique number, and therefore we were able to give each genotype a unique number. For example, $\mathrm{AaBbDdEe}$ was assigned 12345678, and A-BBDdee was assigned 10335688 . The genotypes with the exception of the $F_{1}$ were generated by the computer.

The first step in the program was to generate all possible genotypes that first appear in the $F_{2}$ generation. Initially the genotypic identifications for the $F_{1}$ generation were read into the computer on punched cards. In handling the identification, each group of eight numbers in the case of four genes was treated as a set. Each number, i.e., each allele, in the set was stored in the core memory in such a way that it alone could be available at any time.

We generated the $\mathrm{F}_{2}$ genotypes in the following manner: The starting point in obtaining all possible combinations of $F_{1}$ genotypes was to introduce two internal "pointers," $\mathrm{P}_{1}$ and $\mathrm{P}_{2}$, with each pointer equal to 1 . The genotypic identification sets stored in the core memory in locations relative to the pointer values were then transferred to new core locations and expanded into all possible combinations, taking eight numbers at a time. Each combination was stored immediately after its generation, and thus upon completion of the expansion process, 256 new sets existed. This array then needed to be searched to determine what genotypes had been generated and what fractional contribution to the $\mathrm{F}_{2}$ generation had been made. Each number in the set of eight had a unique storage location, but we needed to generate a single identification number for each set (genotype). This was accomplished by adding 1 to each member of the set and then successively multiplying the eight new numbers together. For example,

storage appearance

$\frac{\text { add } 1, \text { multiply }}{2 \times 3 \times 4 \times 5 \times 6 \times 7 \times 8 \times 9}$ $\frac{\text { result }}{362,880}$.

Since each identifier was unique, the result after multiplication was unique. This result was stored in one core location and thereby provided an integer number for cross-reference to the original alphabetical identification and also a single number for future computations. We then compiled a table ${ }^{3}$ of these results. The identification number of each set (genotype) was compared with all existing identification numbers in the table. If a matching number was found, this meant that this particular genotype had been previously generated and stored; if not, the result was stored in the next available position in the table.

To determine what fractional contribution

${ }^{3}$ Refers to computer programing here and to diagram of general program flow (fig. 1, Appendix), not to text tables in this report. 
had been made, results in the expansion, consisting of the 256 sets in the case of four genes, had to be checked to determine if a particular identification number had previously appeared in the expansion. When the expansion was completed, a variable (IRES) was set equal to the first member of the set. A second variable (ADDER) was set to zero. Each member of the set was then compared to IRES. If a matching member was found, ADDER was incremented by 1 , and the member of the set on which the match had been made was set to zero to prevent further matchings. After all members had been checked against IRES, the variable ADDER was stored in a table at a location corresponding to the location in the table where the result of the multiplication (identification number) had been stored. The variables $P_{1}$ and $P_{2}$ were also stored in corresponding table locations in order to determine what matings had contributed the previously stored fractions to the $\mathrm{F}_{2}$ generation.

Control was then returned to checking the remainder of the 256 sets. The variable IRES was set equal to the next nonzero member of the set, ADDER was set to zero, and the previously described program steps were executed until all 256 sets had been accounted for. Control was then returned to the original expansion process, and the variable $P_{2}$ was increased by 1 . This process continued until all possible combinations of the $F_{1}$ genotypes had been made. This completed the first step of the program.

The second phase was to generate from the previous results similar tables that would be used in making the final computations for the $\mathrm{F}_{3}$ and $\mathrm{F}_{4}$ generations. Since all possible genotypes had already appeared, their generation for the next set of tables was not necessary. All combinations of the $\mathrm{F}_{2}$ genotypes could be made, and each member could be directly checked against the existing table until a location relative to the first position was found by matching. Because of the massive number of combinations and resulting fractional contributions (up to 2.5 million), we could not store these numbers in core memory. The tables were written on direct-access storage devices, commonly called disk storage or disk files.

To minimize the number of searches required to find a particular matching, the existing table was first sorted into numerical ascending order. Matchings were found by executing a binary search on the table. This reduced the average number of searches from 41 (81 maximum, one minimum) to four (seven maximum, one minimum) and considerably increased our execution speed.

As before, two pointers, $\mathrm{P}_{3}$ and $\mathrm{P}_{4}$, were introduced and the process of expansion was begun. The results were intermediately stored in core memory buffer areas. When a buffer was filled, the entire contents were written into the proper disk file location, and this again resulted in increased execution speed. When this phase of the program was completed, the only remaining step was to actually compute the number of offspring or relative frequency for a particular genotype. A set of variables consisting of release ratios, numbers of offspring per mating, and levels of sterility were read into core memory on punched cards. The values for numbers of offspring for the $F_{1}$ generation were then computed from formulas that were entered at the time the source program was compiled.

The process of computing the $F_{2}$ and higher generations was then accomplished by referring to the previously generated tables. Three pointers were again introduced, $\mathrm{P}_{1}$ to 1 and the remaining two $\left(\mathrm{P}_{2}\right.$ and $\left.\mathrm{P}_{3}\right)$ to zero. Pointer $\mathrm{P}_{1}$ was used to point to the table location of the particular genotype that was to be generated, whereas $\mathrm{P}_{2}$ and $\mathrm{P}_{3}$ were set equal to the previously stored table values that identified which two genotypes had been crossed to produce a particular fractional contribution to the genotype being generated. Since the values for the number of $F_{1}$ offspring were generated in the same order and the same relative storage locations were maintained as in the previously generated tables, the following formula could be applied:

Offspring $\left(\mathrm{F}_{2}\right)$ (relative location in memory determined by $\mathrm{P}_{1}$ ) $=$ (contribution/ 256) (fractional contribution stored in core memory and accessed by $\left.\mathrm{P}_{1}\right) \times\left(\mathrm{F}_{1}\right.$ offspring $\left.\left(\mathrm{P}_{2}\right)\right)\left(\mathrm{P}_{2}\right.$ used to locate appropriate $F_{1}$ value $) \times\left(F_{1}\right.$ offspring $\left.\left(P_{3}\right)\right)\left(P_{3}\right.$ used to locate second appropriate $F_{1}$ value)

Therefore offspring $\left(\mathrm{F}_{2}\right)=$ (contribution $\left(\mathrm{P}_{1}\right) /$ $256) \times\left(F_{1}\left(P_{2}\right)\right) \times\left(F_{1}\left(P_{3}\right)\right)$. The value for 
the $\mathrm{F}_{2}$ offspring was then weighted accordingly.

The process for deternining the numbers of $F_{3}$ and $F_{4}$ offspring was identical except that the tables containing fractional contributions and relative locations were stored on direct-access storage devices.

The final phase was to print the results. The results of the computation process were stored in core memory and were written on the printer only when the $F_{1}, F_{2}, F_{3}$, and $F_{4}$ computation processes were completed. Control was then returned to the point in the program where computations were made based on the previously computed formulas, using a new particular weighting factor.

All programs written for this study had this same basic form and were executed in a similar manner. The number of genes involved dictated the amount of core storage required, the number of genotypes generated, and the speed of execution of the entire program.

Using these computational methods, we calculated the theoretical population trends (tables $3-14)$ when a native population of 2,000 insects (1:1 sex ratio) was overflooded by partially sterile insects. The native and release strains differed by one to four genes, and in some instances one of the four genes was sex-linked. The rate of increase between generations was either fivefold or tenfold. Sterility of both sexes of the release strain was either 80 or 90 percent. Four releases were made to coincide with the parental, $F_{1}, F_{2}$, and $F_{3}$ generations. Each release consisted of either 198,000 or 396 , 000 insects (1:1 sex ratio). Matings between partially sterile males and partially sterile females were assumed to yield either no offspring or a number of offspring proportionate to their level of sterility.

In tables $3-14$ the number of individuals for each genotype is rounded to the nearest whole number. However, the column totals were computed before the numbers had been rounded.

To illustrate how populations may be suppressed with genes for conditional lethal traits while the populations are held in check with partial sterility, we derived tables $16-27$ from tables 3-14. (Actually the number of individuals in tables 16-27 were obtained directly from the computer printout for tables 3-14. In this way rounding errors were minimized.) Table 15 gives the interpretations and transscriptions and the method required to derive tables 16-27 from tables $3-14$. The values in tables 3-14 were obtained by introducing insects with all alleles designated by capital letters into populations with all alleles designated by lower case letters. When this was not so, certain transcriptions of lower case letters to capitals were made in order to obtain the appropriate genotypic frequencies from tables 3-14. Tables 16-27 are summarized in table 28. In establishing table 28, we arbitrarily assumed that complete suppression would be achieved when the population of individuals with viable genotypes was reduced to 30 . The eight cases in table 28 are as follows:

\begin{tabular}{|c|c|c|c|}
\hline Case & $\begin{array}{l}\text { Number } \\
\text { released }\end{array}$ & $\begin{array}{l}\text { Sterility } \\
\text { (percent) }\end{array}$ & $\begin{array}{l}\text { Rate of } \\
\text { increase }\end{array}$ \\
\hline 1. & & 90 & Fivefold. \\
\hline & & 80 & Do. \\
\hline $\begin{array}{l}3 \\
4\end{array}$ & 198,000 & $\left\{\begin{array}{l}90 \\
80\end{array}\right.$ & $\begin{array}{l}\text { Tenfold. } \\
\text { Do. }\end{array}$ \\
\hline & & 90 & Fivefold. \\
\hline & 396,000 & 80 & Do. \\
\hline f & 050,000 & $l_{80}^{90}$ & $\begin{array}{c}\text { Tenfold. } \\
\text { Do. }\end{array}$ \\
\hline
\end{tabular}

The following data about complete suppression are indicated in table 28 :

(1) Polyfactorial inheritance of a conditional lethal trait may be more or less advantageous from the standpoint of complete suppression than when the trait is inherited as a single fully dominant gene, depending on the number of native-type alleles required to prevent expression of the lethal trait.

(2) The more lethal traits incorporated in the release strain, the greater is the approach to complete suppression.

(3) Complete suppression cannot be achieved when the number of insects released in each generation is 198,000 , sterility is 80 percent, and the rate of increase between generations is tenfold (case 4), except in the $F_{4}$ generation when the release strain possesses three lethals (two are dominant autosomals and the third is bifactorial, requiring three native alleles to prevent expression) and when matings between partially sterile insects yield offspring proportionate to the level of sterility. 
(4) The number of generations per year may profoundly affect the likelihood of complete suppression. When matings between partially sterile insects produce no offspring, the more generations per year (hence the more releases made), the greater is the likelihood for complete suppression. Generally this is also true when matings between partially sterile insects yield a number of offspring proportionate to their levels of sterility. However, when the release strain has one conditional lethal trait inherited as two autosomal genes (four native alleles required for survival) or three autosomal genes (five native alleles required for survival), then complete suppression would be achieved if the trait was expressed in the $\mathbf{F}_{3}$ generation but not if the expression was delayed to the $F_{4}$ when 396,000 insects are released per generation that are 90 percent sterile and when the rate of increase is tenfold (case 7).

(5) When one of the traits is determined by a dominant gene on the sex chromosomes, the number of male survivors may differ widely from the number of female survivors. Doubtless the low probability of females finding a mate when the density of males is low would have an important role in complete suppression.

If matings between partially sterile insects yield offspring partially or fully proportionate to the level of sterility, the population may become large and threaten damage to the economic host. This consideration, along with adverse effects of sterilization procedures and economic factors, should be taken into account in deciding whether the population to be completely suppressed should be held in check by partial sterility or by conventional means. In lepidopterous species partial sterility is inherited $(8-10)$. In such species the use of partial sterility would be much more effective than indicated in our calculations in facilitating the introduction of genes for conditional lethal traits. Similar considerations would apply if the release insects differed from the native insects by one or more reciprocal chromosomal translocations.

\section{Suppression of Population Held Static by Conventional Means With Conditional Lethal Traits}

By suitable means such as application of insecticides, a population of insects may be prevented from increasing. For example, a population that increases fivefold between generations may be held static by destroying 80 percent of each generation. We calculated the effects of overflooding only the parental (spring) generation with a strain homozygous for one, two, or three conditional lethal traits. The population is assumed to remain static in all subsequent generations. We calculated the relative frequencies of each genotype in the $F_{1}$, $\mathrm{F}_{2}, \mathrm{~F}_{3}$, and $\mathrm{F}_{4}$ generations by using the general methods already outlined. However, the number of insects with each genotype was divided by the total number of insects. For purposes of computation, we assumed that each mated female produced one pair of offspring and the insects were fully fertile. These values are shown in tables 29-34.

To understand the significance of these figures, consider a field of 1,000 acres with 10 insects per acre, or a total of 10,000 insects. This low population for a number of economic pests such as the boll weevil (Anthonomus grandis Boheman), pink bollworm (Pectinophora gossypiella (Saunders)), and perhaps others is feasible and is held static, or at least is not permitted to reach economic density levels, throughout the season by using insecticide applications or other available control measures. We can calculate the effect on this population when the conditional lethal traits are expressed. The number of survivors is the product of the number of insects times the relative frequency of genotypes capable of surviving. In this example we obtain the frequency of viable genotypes from tables 29-34 and multiply this frequency by 10,000 . We calculated cases $1 \mathrm{a}-15 \mathrm{~b}$ in table 41 in order to illustrate the use of tables 29-34 and to demonstrate the potential of conditional lethal traits for population suppression. The method used to derive table 41 is shown in table 15 .

Case 1 in table 41 was calculated by using table 29. With one gene the genotypic frequencies do not change from generation to genera- 
tion. If 500 insects were released per acre to give a release ratio of $50: 1$ and if the total population (native plus released) was held static thereafter, four insects would be capable of surviving when the conditional lethal trait would be expressed in the $F_{2}$ or subsequent generations. Whether this level of survival would result in eradication would depend on the species concerned, but in all likelihood so few insects on 1,000 acres would represent eradication. In actual practice a judgment would have to be made concerning the minimum density required for survival. After this judgment is made, the release ratio required for complete suppression can be obtained from table 29 .

Examination of cases 2-6 in table 41 shows that when a conditional lethal trait is determined by more than one gene, the trait may be expressed in a progressively higher proportion of individuals from generation to generation. Cases $7-15$ show that when the release strain possesses more than one conditional lethal trait, the efficiency of suppression is progressively augmented in succeeding generations. If the conditional lethal traits were expressed in the last generation of the season, release ratios ranging from $10: 1$ to $50: 1$ would be adequate to decimate most populations with four generations per year. This would require releases ranging from 100 to 500 insects per acre when the natural population consists of 10 insects per acre. Higher ratios would be required if the species had fewer than four generations per year. In most instances these ratios would have to be two to five times higher to achieve the same suppression in the $F_{2}$ as in the $F_{4}$.

If a conditional lethal trait is under the control of more than one gene in a species with two generations per year, conditional lethal genes would be about one to 10 times more efficient than dominant lethal mutations in suppressing insect populations. If the species has three generations per year, conditional lethal genes would be up to 100 times more efficient than dominant lethal mutations. Since the release of insects carrying dominant lethal mutations is considered feasible for eradicating a number of economic insects existing at low population levels, the practical significance of the condi- tional lethal genes, if such can be found and used, is readily apparent.

We calculated the effects of overflooding a native population once every generation with a strain homozygous for one, two, or three conditional lethal traits. We assumed that appropriate measures would be taken to hold the population constant in all generations. We calculated the relative frequencies of each genotype in the $F_{1}, F_{2}, F_{3}$, and $F_{4}$ generations, and these values are shown in tables $35-40$.

To understand the significance of these figures, we will again consider a field of 1,000 acres with 10 insects per acre, or a total of 10,000 insects. This population is held static throughout the season with insecticide applications or other suitable means. We calculated the effect on this population when the conditional lethal traits are expressed. The number of survivors is 10,000 times the relative frequency of the genotypes capable of surviving. The latter data were obtained from tables 35-40. To demonstrate the potential of conditional lethal traits for population suppression and to illustrate the use of tables $35-40$, we then calculated cases $16 a-30 b$, as shown in table 41 .

Case $16 \mathrm{a}$ in table 41 was calculated by using table 35 . If 100 insects are released per acre during each generation (release ratios of 10:1), the frequency of viable genotypes would rapidly decline from 83 in the $F_{1}$ to 0 in the $F_{4}$. Comparison of case $16 \mathrm{a}$ with cases $17-21$ shows that the efficiency of suppression may be significantly greater when a conditional lethal trait is under the control of several genes than when the trait is determined by a single fully dominant factor. Cases 22-30 show that when the release strain possesses more than one conditional lethal trait, the release ratio required to destroy the population is much smaller than when the release strain possesses only one conditional lethal trait.

Release ratios ranging from $1: 1$ to $10: 1$ would be adequate to decimate most populations with four generations per year if the conditional lethal traits are expressed in the last generation. In many instances such low ratios would be adequate for species with two generations per year but not for those with one. To sup- 
press a population with just two generations per year to the same extent as a population with four generations per year, we would have to release three to 10 times as many insects per release as are required to suppress a population with four generations per year.

\section{Discussion}

The preceding calculations indicate that conditional lethal traits could be very effective in suppressing insect populations. The greatest advantage of using adaptations to climate or seasonal changes as conditional lethal traits is that these traits already exist in populations at latitudes or altitudes different from those of the locality where we desire complete suppression.

To assess the possibility of utilizing these adaptations to suppress an insect population, one would collect a strain from a locality with a distinctly different season, select the strain to apparent homozygosity for one or more traits, cross the latter to the strain from the locality designated for suppression, and determine the expression of the traits in hybrid generations. Since temperature may modify responses to photoperiod (2), the expression of the traits in hybrid generations should be checked in an environmental chamber programed to simulate conditions in the field. In this way one could detect undesirable genotypes in the release strain. Although desirable, one need not determine precisely the inheritance of each trait. The investment required to assess the feasibility of this proposed suppression method in any particular instance would not be prohibitive.

\section{Literature Cited}

(1) Commonwealth Institute of Entomology. 1951-69. DISTRIBUTION MAPS OF INSECT PESTS. Series A 41, 228 pp. London.

(2) Danilevskit, A. S.

1965. PHOTOPERIODISM AND SEASONAL DEVELOPMENT OF INSECTS. $283 \mathrm{pp}$. London.

(3) - ed.

1968. PHOTOPERIODIC ADAPTATIONS TO INSECTS AND ACARI. $269 \mathrm{pp}$. Leningrad University.

(4) Hogan, T. W.

1966. PHYSIOLOGICAL DIFFERENCES BETWEEN RACES OF TELEOGRYLLUS COMMODUS (WALKER) (ORTHOPTERA: GRYLLIDAE) RELATED TO A PROPOSED GENETIC APPROACH To CONTROL. Austral. Jour. Zool. 14: 245-251.

(5) Klassen, W., Knipling, E. F., and McGuire, J. U. 1970. THE POTENTIAL FOR INSECT POPULATION SUPPRESSION BY DOMINANT CONDITIONAL Lethal Traits. Ent. Soc. Amer. Ann. $63: 238-255$.
(6) MASAKI, S.

1961. GEOGRAPHIC VARIATION OF DIAPAUSE IN InseCts. Hirosaki Univ. Facul. Agr. Bul. $7: 245-251$.

(7)

1968. GEOGRAPHIC ADAPTATION IN THE SEASONAL LIFE CYCLE OF MAMESTRA BRASSICAE (LINNE) (LEPIDOPTERA: NOCTUIDAE). Hirosaki Univ. Facul. Agr. Bul. 14: 16-26.

(8) North, D. T., and HolT, G.

1968. INHERITED STERILITY IN PROGENY OF IRRADIATED MALE CABBAGE LOOPERS. Jour. Econ. Ent. 61: 928-931.

(9) Proverbs, M. D., and Newton, J. R.

1962. SOME EFFECTS OF GAMMA RADIATION ON THE REPRODUCTIVE POTENTIAL OF THE CODLING MOTH CARPOCAPSA POMONELLA (L.) (LEPIDOPTERA: OLETHRUETIDAE). Canad. Ent. 94: 1162-1170.

(10) Walker, D. W., and Pedersen, K. B.

1969. POPULATION MODELS FOR SUPPRESSION OF THE SUGARCANE BORER BY INHERITED PARtial Sterility. Ent. Soc. Amer. Ann. 62: 21-26.

\section{Appendix}

The following method is used to calculate the genotypic frequencies in hybrid generations when a native population (AABB) is over- flooded with a partially sterile release strain (aabb) in the parental, $\mathbf{F}_{\mathbf{1}}, \mathbf{F}_{2}$, and $\mathbf{F}_{\mathbf{3}}$ generations. 

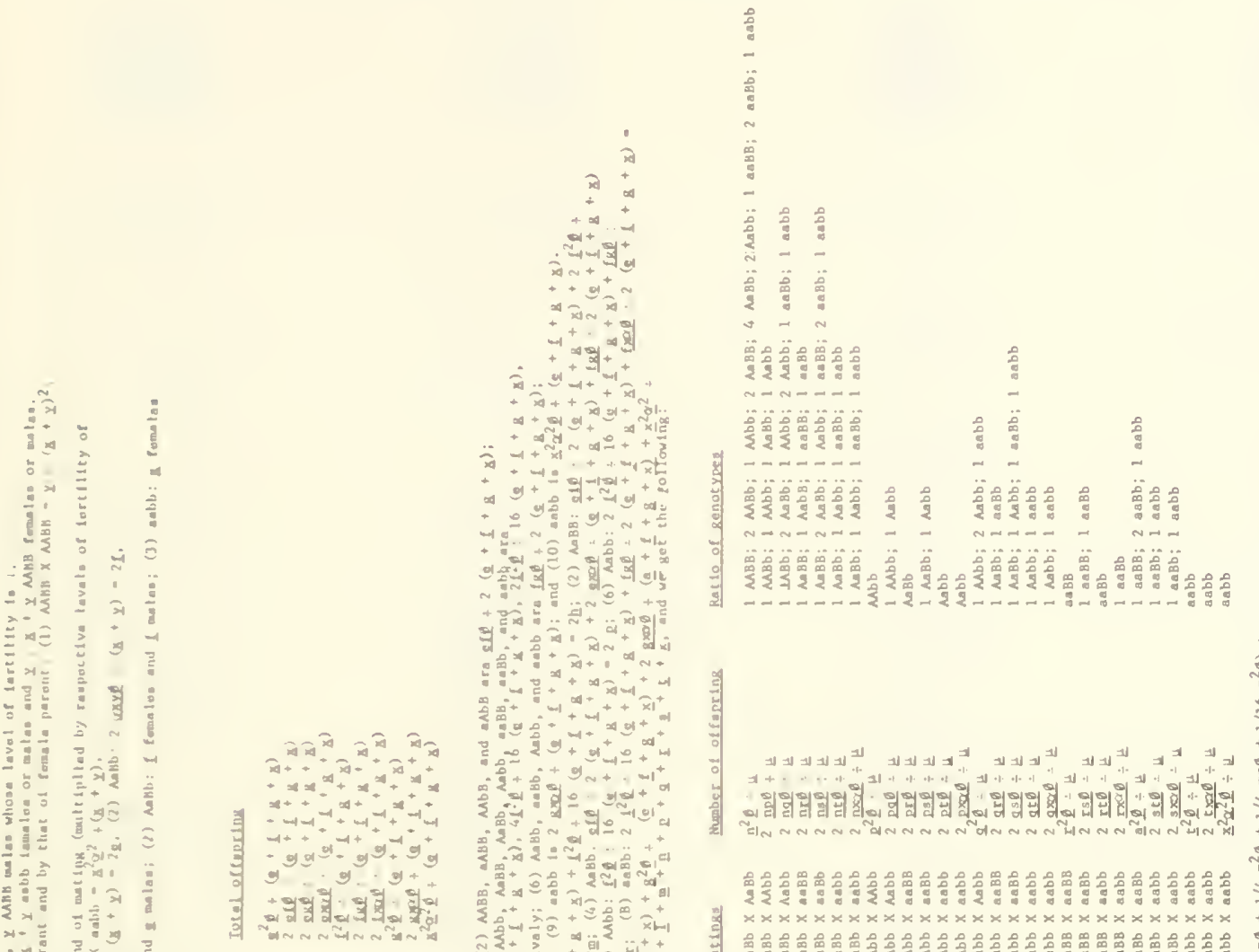

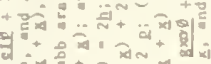

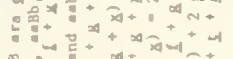

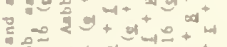

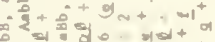

₹ैं

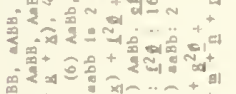

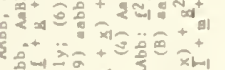

제

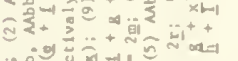

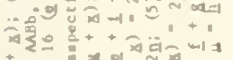

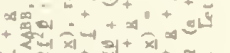

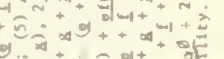

de.

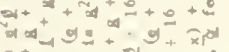

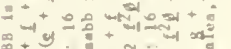

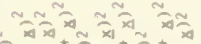

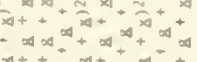

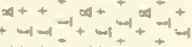

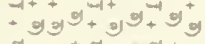

-

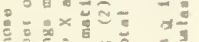

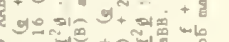

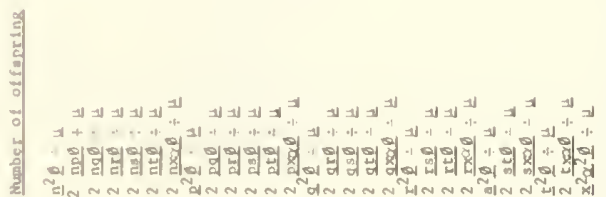

काष

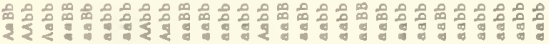

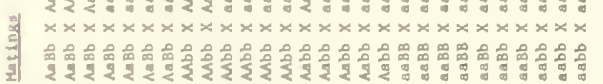

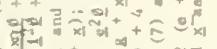

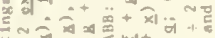

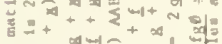

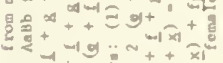

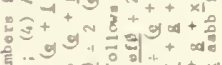
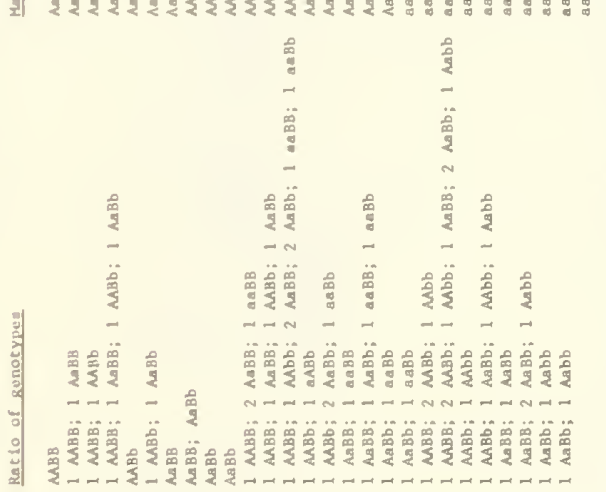

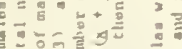

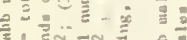

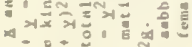

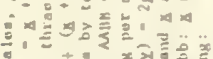

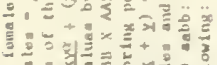

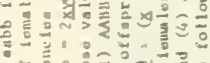

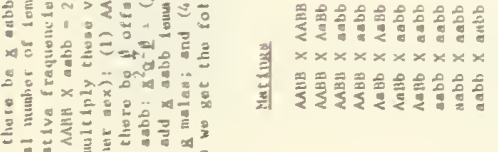

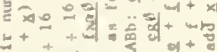

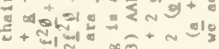

音

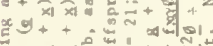

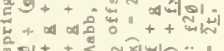

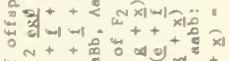

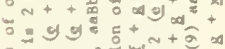

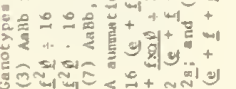

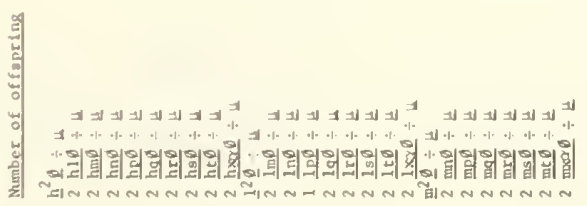

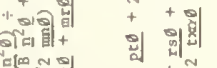

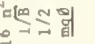

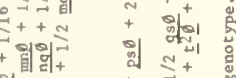

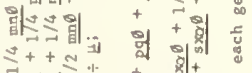

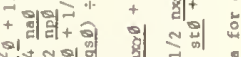

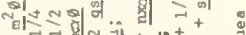

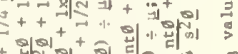

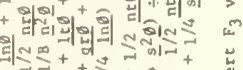

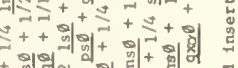

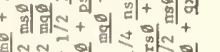

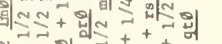

+

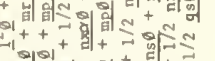

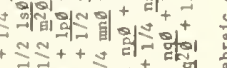

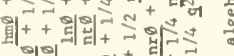
กำ

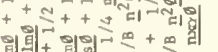

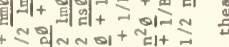

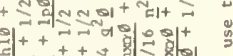

过+++ +

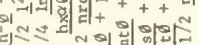

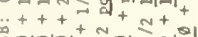

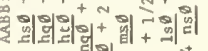

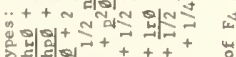
a
and

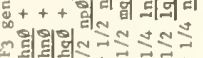

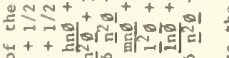

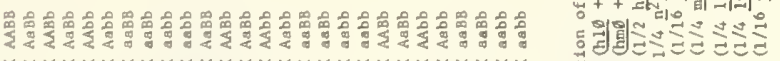

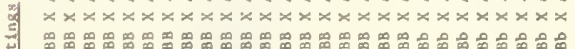

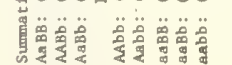




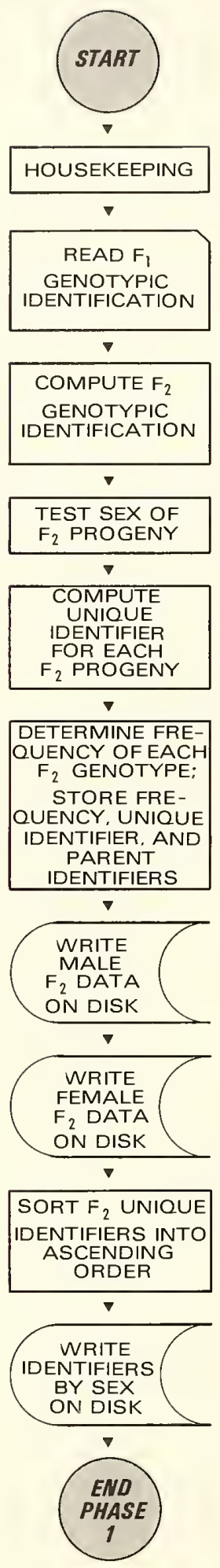

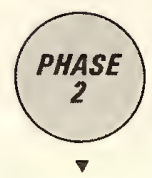
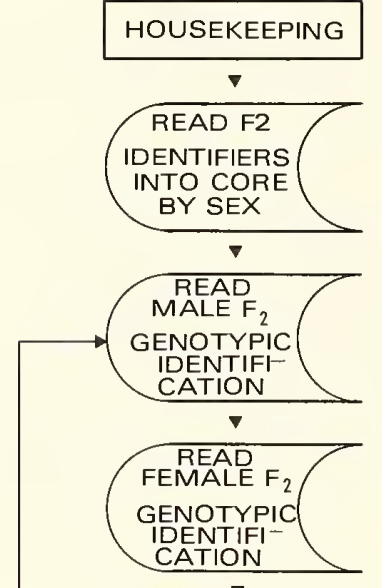

COMPUTE $F_{3}$ GENOTYPIC IDENTIFICATION
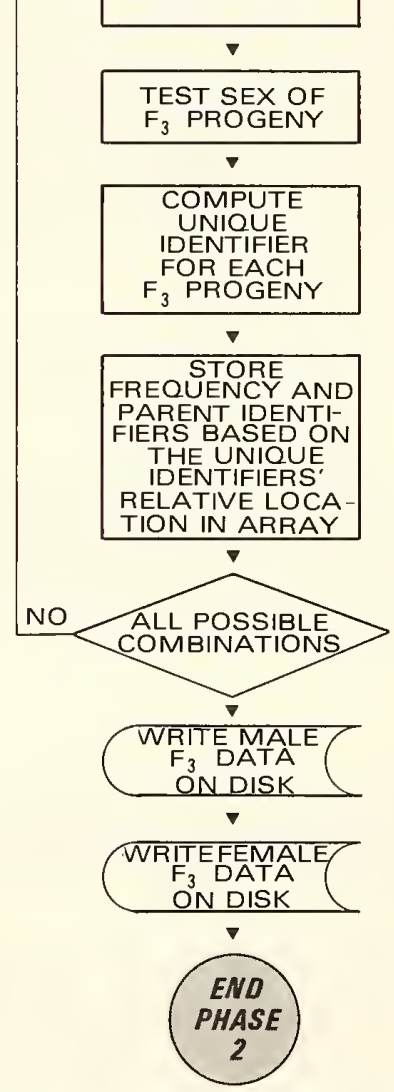

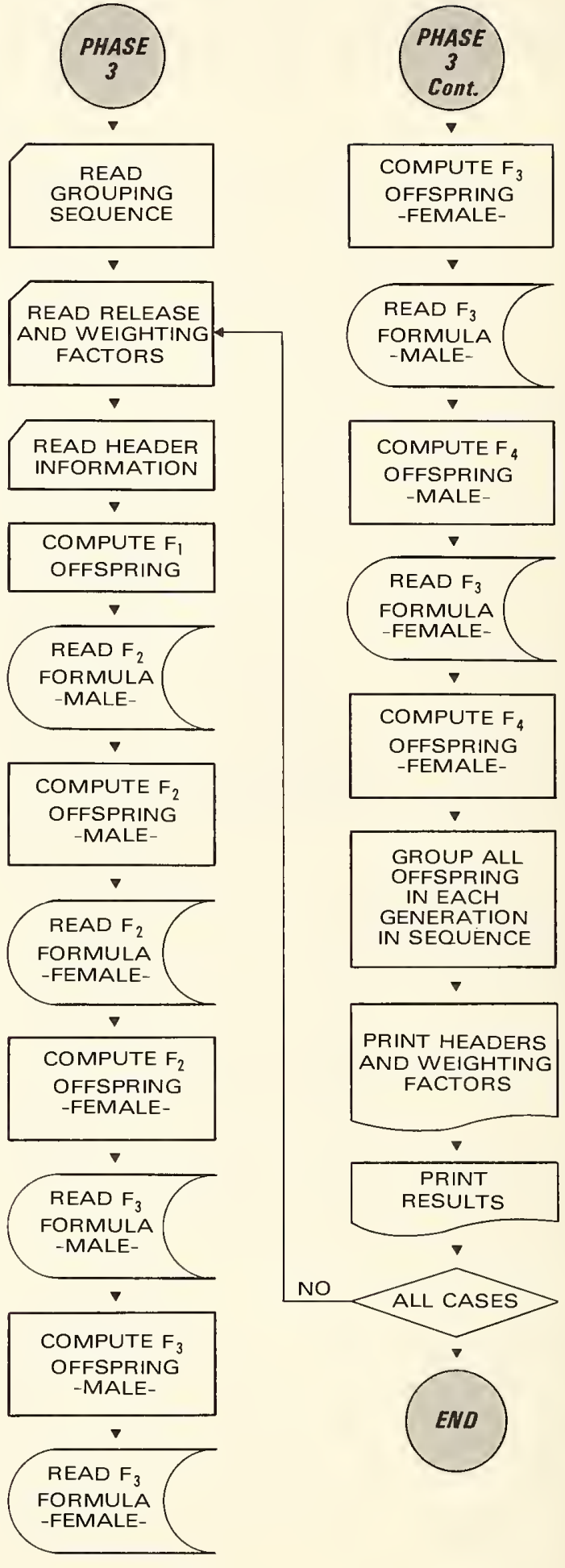

Figure 1.-General program flow and techniques used to develop computer programs. 


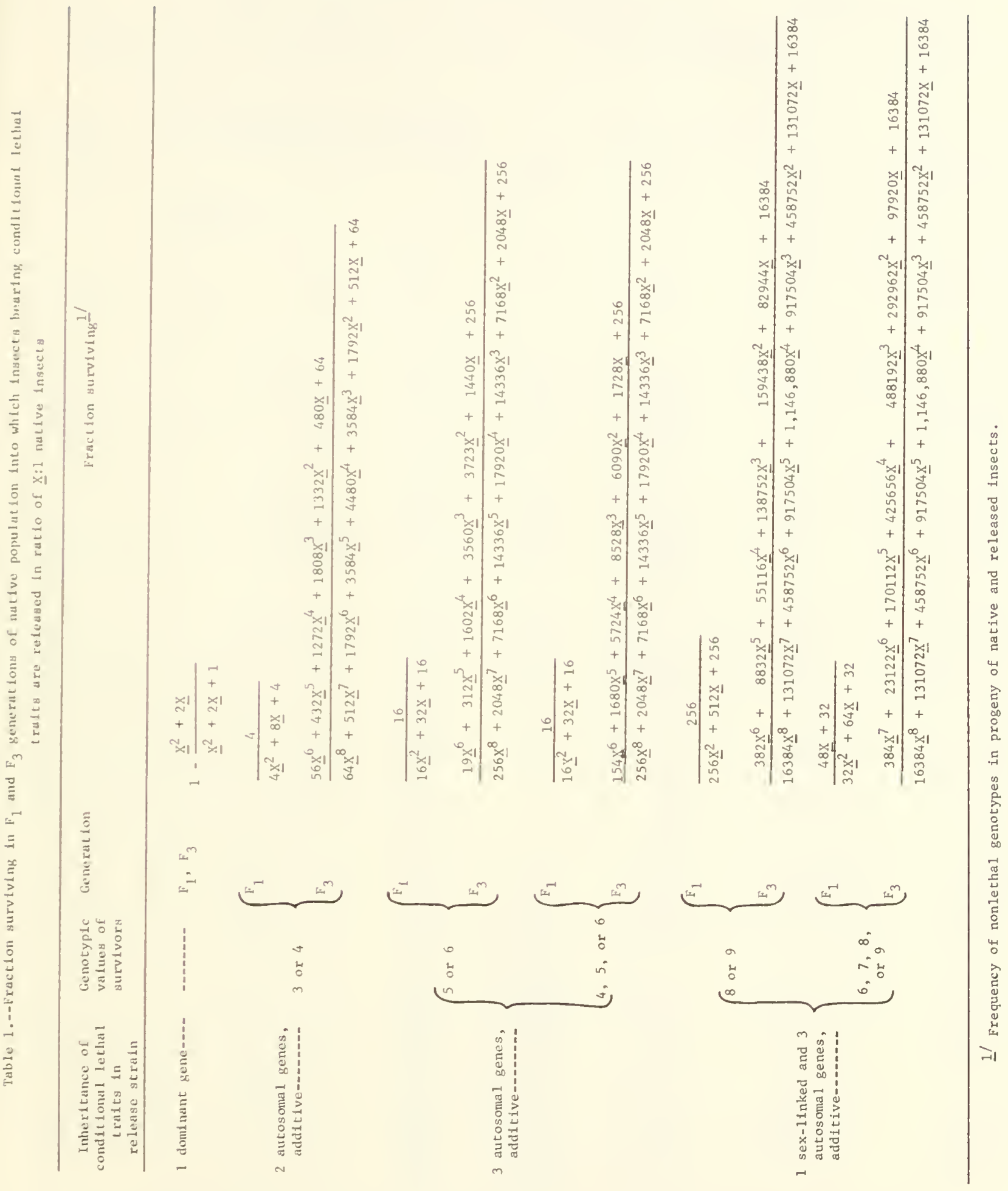


Table 2.--Release ratios required for 98- and 99.9-percent suppression in $F_{1}$ and $F_{3}$ generations of native population overflooded in parental (overwintered) generation with release strain bearing conditional letha1 traits

\begin{tabular}{|c|c|c|c|c|c|c|c|c|}
\hline \multirow{2}{*}{\multicolumn{5}{|c|}{$\begin{array}{l}\text { Inheritance of conditional lethal traits } \\
\text { in release strain } 1\end{array}$}} & \\
\hline & & & & & \multicolumn{4}{|c|}{$\begin{array}{cc}98 \text {-percent } & 99.9-\text { percent } \\
\text { suppression in-- } & \text { suppression in-- }\end{array}$} \\
\hline & Trait 1 & & Trait 2 & Trait 3 & $\mathrm{~F}_{1}$ & $\mathrm{~F}_{3}$ & $\mathrm{~F}_{1}$ & $\mathrm{~F}_{3}$ \\
\hline & dom.auto. & -- & & --- & 49 & 49 & 1,000 & 1,000 \\
\hline & auto. $(3,4)$ & -- & & ---- & 49 & 48 & 1,000 & 1,000 \\
\hline & auto. $(5,6)$ & -- & & ---- & 49 & 8 & 1,000 & 84 \\
\hline & auto. $(4-6)$ & --- & & --- & 49 & 36 & 1,000 & 1,000 \\
\hline & auto. $+1 \mathrm{~s} .1$ & & & & & & & \\
\hline & $(8,9)$ & -- & & --- & 49 & 5 & 1,000 & 36 \\
\hline & $\begin{array}{c}\text { auto. }+1 \text { s. } 1 \\
(6-9)\end{array}$ &.- & & --- & $>1,000$ & $>1,000$ & $>1,000$ & $>1,000$ \\
\hline & dom.auto. & & auto. $(3,4)$ & --- & 49 & 3 & 1,000 & 10 \\
\hline & dom. auto. & & auto. $(5,6)$ & --- & 49 & 2 & 1,000 & 6 \\
\hline & dom.auto. & & auto. $(4-6)$ & --- & 49 & 3 & 1,000 & 9 \\
\hline & dom. auto. & 3 a & $\begin{array}{l}\text { auto.+ } 1 \text { s.1. } \\
(8,9)\end{array}$ & $-\cdots$ & 49 & 2 & 1,000 & 5 \\
\hline & dom.auto. & $1 d$ & Hom. auto & ---- & 49 & 3 & 1,000 & 10 \\
\hline 1 & dom, auto. & $3 a$ & $\underset{(6-9)}{\text { auto. }} 1$ s. 1 & --- & 49 & 4 & 1,000 & 11 \\
\hline 2 & auto. $(3,4)$ & & auto. $(3,4)$ & --- & 49 & 4 & 1,000 & 10 \\
\hline 2 & auto. $(3,4)$ & & auto. $(5,6)$ & --- & 49 & 3 & 1,000 & 6 \\
\hline 2 & auto. $(3,4)$ & & auto. $(4-6)$ & ---- & 49 & 4 & 1,000 & 9 \\
\hline 2 & auto. $(3,4)$ & $3 a$ & $\begin{array}{l}\text { auto.+ } 1 \text { s. } 1 \text {. } \\
(8,9)\end{array}$ & --- & 49 & 2 & 1,000 & 5 \\
\hline 2 & auto. $(3,4)$ & 3 a & $\begin{array}{l}\text { auto. }+1 \text { s.1. } \\
(6-9)\end{array}$ & $-\cdots$ & 49 & 4 & 1,000 & 11 \\
\hline 3 & auto. $(5,6)$ & & auto. $(5,6)$ & $\cdots$ & 49 & 2 & 1,000 & 4 \\
\hline 3 & auto. $(5,6)$ & & auto. $(4-6)$ & $\ldots-$ & 49 & 3 & 1,000 & 6 \\
\hline 3 & auto. $(5,6)$ & & auto. $(4-6)$ & $-\cdots$ & 49 & 4 & 1,000 & 9 \\
\hline 3 & auto. $(5,6)$ & $3 a$ & $\begin{array}{l}\text { auto.+ } 1 \text { s. } 1 \text {. } \\
(8,9)\end{array}$ & --- & 49 & 2 & 1,000 & 3 \\
\hline 3 & auto. $(5,6)$ & 3 a & $\begin{array}{l}(6-9) \\
(6+1 \text { s. }\end{array}$ & --- & 49 & 3 & 1,000 & 7 \\
\hline 3 & auto. $(4-6)$ & 3 a & $\begin{array}{l}\text { auto. }+1 \text { s.1. } \\
(8,9)\end{array}$ & 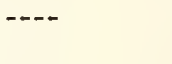 & 49 & 2 & 1,000 & 5 \\
\hline 3 & auto. $(4-6)$ & 3 a & $\begin{array}{l}\text { auto. }+1 \text { s. } 1 . \\
(6-9)\end{array}$ & $-\cdots$ & 49 & 4 & 1,000 & 11 \\
\hline & $\begin{array}{l}\text { auto. }+1 \text { s. } 1 \\
(8,9)\end{array}$ & 3 a & $\begin{array}{l}\text { auto.+ } 1 \text { s.1. } \\
(8,9)\end{array}$ & $\ldots$ & 49 & 2 & 1,000 & 3 \\
\hline 3 & $\begin{array}{c}\text { auto. }+1 \text { s.1. } \\
(8,9)\end{array}$ & 3 a & $\begin{array}{l}\text { auto.+ } 1 \text { s.1. } \\
(6-9)\end{array}$ & $\cdots$ & 49 & 2 & 1,000 & 5 \\
\hline & $\begin{array}{l}\text { auto. }+1 \text { s. } 1 . \\
(6-9)\end{array}$ & 3 a & $\begin{array}{c}\text { auto. }+1 \text { s.1. } \\
(6-9)\end{array}$ & 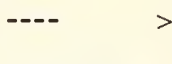 & $>1,000$ & 5 & $>1,000$ & 14 \\
\hline 1 & dom. auto. & $1 \mathrm{c}$ & dom.auto. & 1 dom.auto. & 49 & 2 & 1,000 & 3 \\
\hline 1 & dom. auto. & & dom. auto. & 3 auto. $(5,6)$ & 49 & 2 & 1,000 & 3 \\
\hline & dom.auto. & $2 a$ & auto. $(3,4)$ & 3 auto. $(4-6)$ & 49 & 2 & 1,000 & 4 \\
\hline 1 & dom.auto. & & auto. $(3,4)$ & $\begin{array}{c}3 \text { auto. }+1 \\
\text { s. } 1 .(6-9)\end{array}$ & 49 & 2 & 1,000 & 4 \\
\hline 2 & auto. $(3,4)$ & 2 & auto. $(3,4)$ & 2 auto. $(3,4)$ & 49 & 2 & 1,000 & 4 \\
\hline 2 & auto. $(3,4)$ & 2 & auto. $(3,4)$ & $\begin{array}{c}3 \text { auto. }+1 \\
\text { s. } 1 .(8,9)\end{array}$ & 49 & 2 & 1,000 & 3 \\
\hline 2 & auto. $(3,4)$ & $3=$ & auto. $(5,6)$ & 3 auto. $(4-6)$ & 49 & 2 & 1,000 & 3 \\
\hline 2 & auto. $(3,4)$ & 3 & auto. $(5,6)$ & $\begin{array}{c}3 \text { auto. }+1 \\
\text { s.1. }(6-9)\end{array}$ & 49 & 2 & 1,000 & 3 \\
\hline 3 & auto. $(5,6)$ & $3=$ & auto. $(5,6)$ & 3 auto. $(5,6)$ & 49 & 1 & 1,000 & 2 \\
\hline 3 & auto. $(5,6)$ & 3 & auto. $(4-6)$ & 3 auto. $(4-6)$ & 49 & 2 & 1,000 & 3 \\
\hline 3 & auto. $(4-6)$ & $3=$ & auto. $(4-6)$ & 3 auto. $(4-6)$ & 49 & 2 & 1,000 & 4 \\
\hline 3 & auto. $(4-6)$ & & $\begin{array}{l}\text { auto. }+1 \text { s.1. } \\
(8,9)\end{array}$ & $\begin{array}{c}3 \text { auto. }+1 \\
\text { s. } 1 .(8,9)\end{array}$ & 49 & 2 & 1,000 & 3 \\
\hline 3 & $\begin{array}{c}\text { auto. }+1 \text { s.1. } \\
(8,9)\end{array}$ & & $\begin{array}{l}\text { auto. }+1 \text { s.1. } \\
(8,9)\end{array}$ & $\begin{array}{r}3 \text { auto. }+1 \\
\text { s. } 1 .(8,9)\end{array}$ & 49 & 1 & 1,000 & 2 \\
\hline 3 & $\underset{(6-9)}{\text { auto. }+1 \text { s.1. }}$ & 3 & $\begin{array}{c}\text { auto.+ } 1 \text { s.1. } \\
(6-9)\end{array}$ & $\begin{array}{r}3 \text { auto. }+1 \\
\text { s.1. }(6-9)\end{array}$ & 49 & 2 & 1,000 & 5 \\
\hline
\end{tabular}


Table 3.--Theoretical population and genotypic trends when native population of 2,000 insects (aa) is overflooded by 198,000 or 396,000 partially sterile insects (AA) during parental, $F_{1}, F_{2}$, and $F_{3}$ generations. Matings between partially sterile insects are assumed to vield no offspring

\begin{tabular}{|c|c|c|c|c|c|c|c|}
\hline \multirow{2}{*}{$\begin{array}{l}\text { Number } \\
\text { released }\end{array}$} & \multirow[t]{2}{*}{ Sterilfty } & \multirow{2}{*}{$\begin{array}{l}\text { Rate of } \\
\text { increase }\end{array}$} & \multirow{2}{*}{$\begin{array}{l}\text { Genera- } \\
\text { tion }\end{array}$} & \multicolumn{4}{|c|}{ Genotype } \\
\hline & & & & $a a$ & $\mathrm{Aa}$ & $\mathrm{AA}$ & Total \\
\hline & Percent & & & & & & \\
\hline $198,000--$ & 90 & Fivefold- & $\left\{\begin{array}{l}F_{1} \\
F_{2} \\
F_{3} \\
F_{4}\end{array}\right.$ & $\begin{array}{r}100 \\
30 \\
9 \\
3\end{array}$ & $\begin{array}{r}1,980 \\
1,133 \\
636 \\
355\end{array}$ & $\begin{array}{r}----- \\
1,005 \\
1,615 \\
2,005\end{array}$ & $\begin{array}{l}2,080 \\
2,168 \\
2,260 \\
2,363\end{array}$ \\
\hline $198,000--$ & 80 & --do-- & $\left\{\begin{array}{l}F_{1} \\
F_{2} \\
F_{3} \\
F_{4}\end{array}\right.$ & $\begin{array}{l}100 \\
107 \\
122 \\
158\end{array}$ & $\begin{array}{l}3,960 \\
4,280 \\
4,978 \\
6,625\end{array}$ & $\begin{array}{r}----- \\
3,977 \\
12,646 \\
33,089\end{array}$ & $\begin{array}{r}4,060 \\
8,364 \\
17,746 \\
39,872\end{array}$ \\
\hline $198,000--$ & 90 & Tenfold-- & $\left\{\begin{array}{l}F_{1} \\
F_{2} \\
F_{3}^{3} \\
F_{4}\end{array}\right.$ & $\begin{array}{l}200 \\
235 \\
322 \\
590\end{array}$ & $\begin{array}{r}3,960 \\
4,697 \\
6,546 \\
12,255\end{array}$ & $\begin{array}{r}4,072 \\
14,275 \\
45,762\end{array}$ & $\begin{array}{r}4,160 \\
9,004 \\
21,143 \\
58,607\end{array}$ \\
\hline $198,000--$ & 80 & --do-- & $\left\{\begin{array}{l}F_{1} \\
F_{2} \\
F_{3} \\
F_{4}\end{array}\right.$ & $\begin{array}{r}200 \\
840 \\
3,991 \\
25,685\end{array}$ & $\begin{array}{r}7,920 \\
17,583 \\
53,351 \\
296,629\end{array}$ & $\begin{array}{r}----- \\
15,977 \\
110,806 \\
813,595\end{array}$ & $\begin{array}{r}8,120 \\
34,400 \\
168,148 \\
1,135,904\end{array}$ \\
\hline $396,000--$ & 90 & Fivefold- & $F_{4} F^{3}$ & $\begin{array}{r}50 \\
14 \\
4 \\
1\end{array}$ & $\begin{array}{r}1,990 \\
1,066 \\
565 \\
298\end{array}$ & $\begin{array}{r}----- \\
1,022 \\
1,557 \\
1,872\end{array}$ & $\begin{array}{l}2,040 \\
2,102 \\
2,126 \\
2,171\end{array}$ \\
\hline $396,000--$ & 80 & --do-- & $\left\{\begin{array}{l}F_{2}^{2} \\
F_{3}^{3}\end{array}\right.$ & $\begin{array}{l}50 \\
52 \\
56 \\
64\end{array}$ & $\begin{array}{l}3,980 \\
4,141 \\
4,477 \\
5,211\end{array}$ & $\begin{array}{r}3,989 \\
12,328 \\
30,514\end{array}$ & $\begin{array}{r}4,030 \\
8,182 \\
16,861 \\
35,789\end{array}$ \\
\hline $396,000--$ & 90 & Tenfold & $\left\{\begin{array}{l}F_{2}^{1} \\
F_{3}^{3} \\
F_{4}\end{array}\right.$ & $\begin{array}{l}101 \\
109 \\
129 \\
178\end{array}$ & $\begin{array}{l}3,980 \\
4,346 \\
5,170 \\
7,242\end{array}$ & $\begin{array}{r}4,038 \\
13,116 \\
35,955\end{array}$ & $\begin{array}{r}4,081 \\
8,493 \\
18,415 \\
43,375\end{array}$ \\
\hline $396,000--$ & 80 & --do-- & $\left\{\begin{array}{l}F_{1} \\
F_{2} \\
F_{3} \\
F_{4}\end{array}\right.$ & $\begin{array}{r}101 \\
412 \\
1,809 \\
9,786\end{array}$ & $\begin{array}{r}7,960 \\
16,800 \\
42,538 \\
173,314\end{array}$ & $\begin{array}{r}15,994 \\
103,891 \\
652,111\end{array}$ & $\begin{array}{r}8,061 \\
33,206 \\
148,238 \\
835,211\end{array}$ \\
\hline
\end{tabular}


유ㅇㅛㅛ

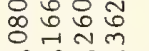

กิก

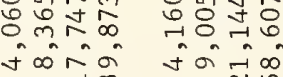

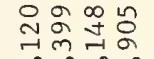

वิ कo

으에

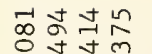

둥ํㅇㅇ

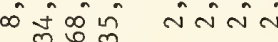

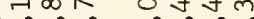

$\infty \stackrel{\rightarrow}{\rightarrow}$

กง

$\infty$ m.

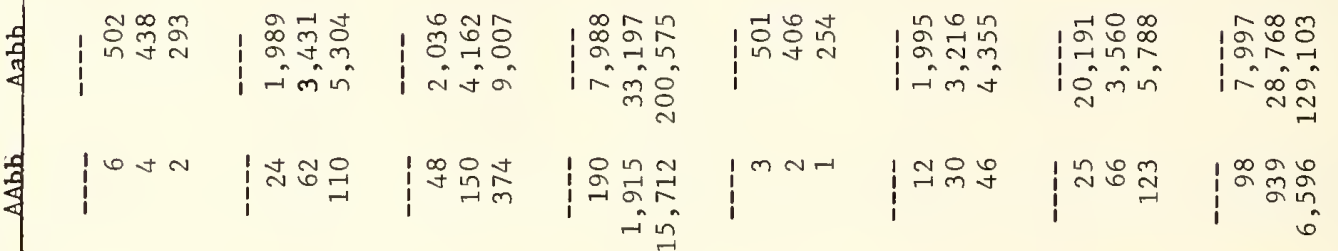

ตี

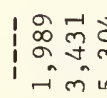

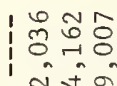

$1 \infty \pi$ in

1

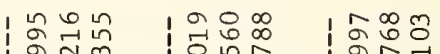

مิ

مू mo

1,

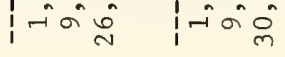

In

สำ

$6+2$

กั่

$\mid \begin{aligned} & \infty \\ & 1 \\ & 1\end{aligned}$

लिं

-imं

ขึ่

กิ

유윰의

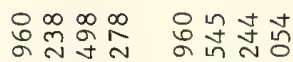

ติก กิ่

낭

नुन

लंतेत्र

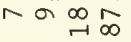

|

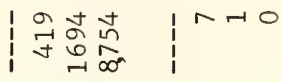

No

$\infty$

$\ln 0 m+\infty a n$

$\stackrel{\operatorname{Ln} ง+}{\sim}$

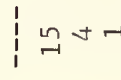

끈유

공워

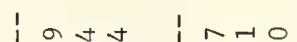

I

ino

|กับำ

in ing

in

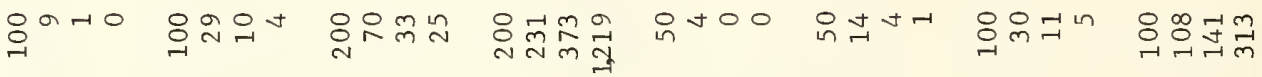

تृ:

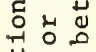

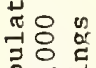

f.

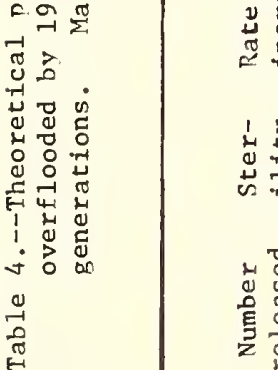

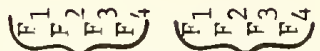

Ninm

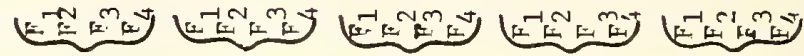




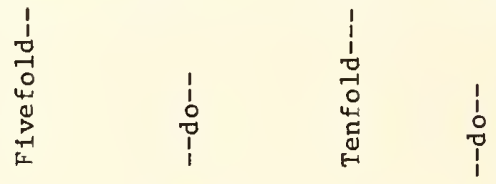

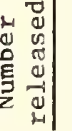

요

$\infty$

은

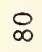

응

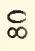

요

$\infty$

1
1
8
0
$\infty$
0
-1

1
1
8
0
$\infty$
0
-1

1
1
8
8
$\infty$
0
-1

1
$\vdots$
8
8
$\infty$
9
-1

1
$\vdots$
8
0
$\check{n}$

1
$\vdots$
8
0
0
m

1
$\vdots$
0
$\vdots$
ह

1
1
8
0
0
0
ले 


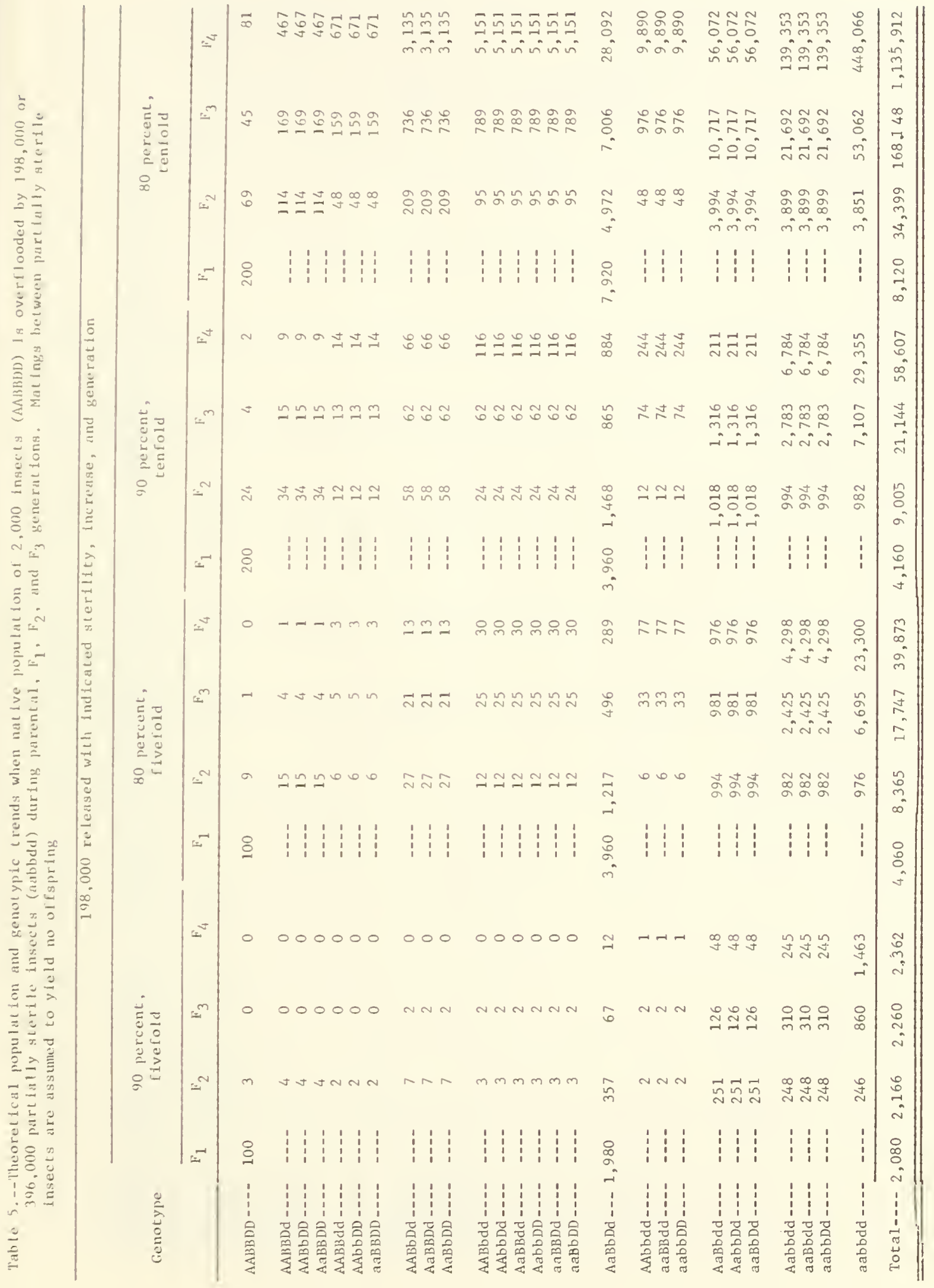




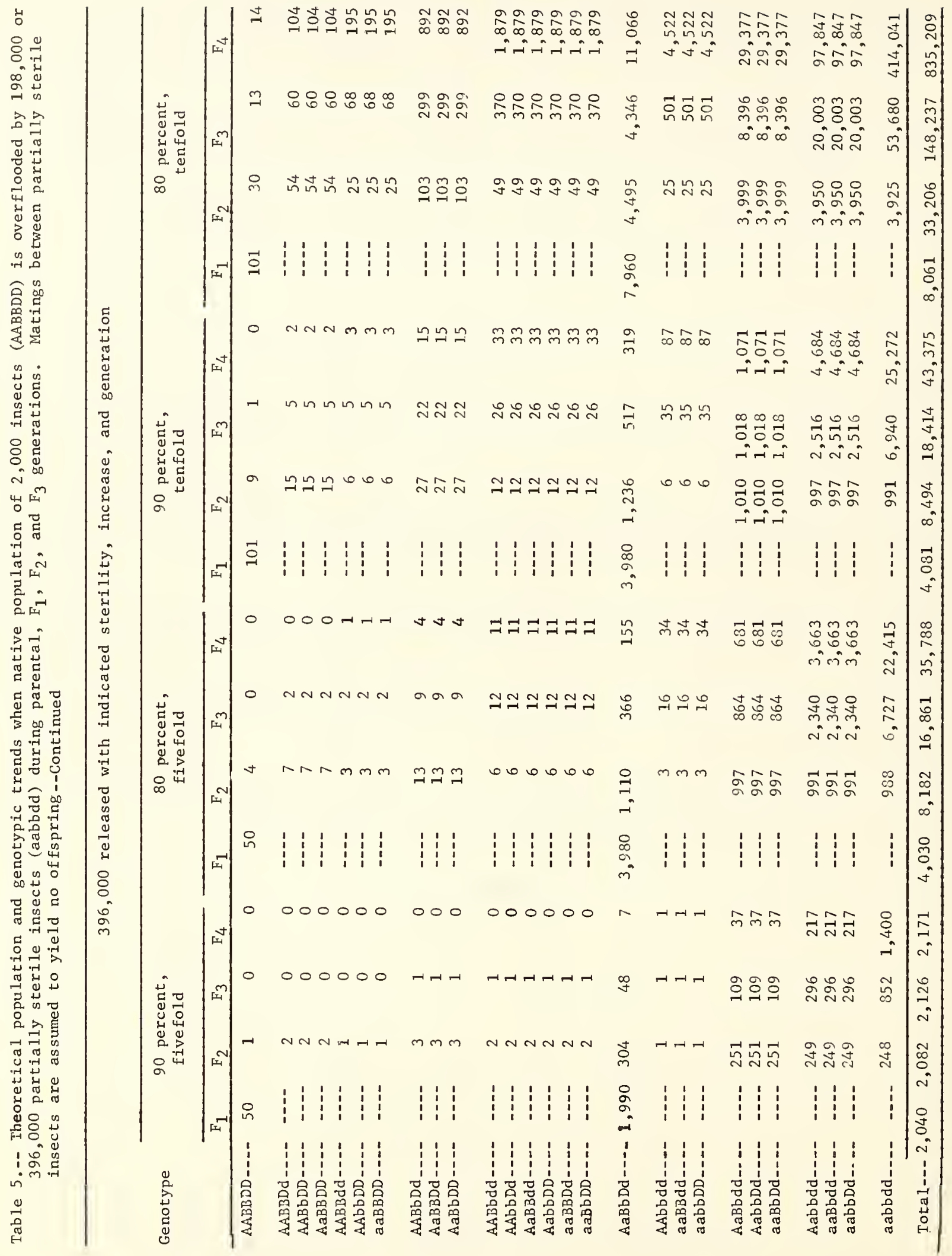


Pabte 6.--Theoretical popularico and genotyple trends when native population of 2,000 Insects (AABBOoEE) is overflooded by 198,000 or 396,000 pertially sterile insects abbddeel during parental, $F_{2}, F_{2}$, and $F_{3}$ generatlons. Matings between partially sterile insects are assued to vield 0 offspring

\begin{tabular}{|c|c|c|c|c|c|c|c|c|c|c|c|c|c|c|c|c|}
\hline venorice & & & $\begin{array}{l}\text { petcen } \\
\text { vefold }\end{array}$ & & & $\begin{array}{l}80 \text { per } \\
\text { flive f }\end{array}$ & $\begin{array}{l}\text { ens, } \\
\text { ld }\end{array}$ & & & $\begin{array}{l}90 \text { pe } \\
\text { ten }\end{array}$ & $\begin{array}{l}\text { cent, } \\
\text { old }\end{array}$ & & & $\begin{array}{l}80 \mathrm{p} \\
\text { te }\end{array}$ & $\begin{array}{l}\text { rcent, } \\
\text { fold }\end{array}$ & \\
\hline & $\because$ & $F_{2}$ & $\mathrm{~F}_{3}$ & $\mathrm{~F}_{4}$ & $F_{1}$ & $\mathrm{~F}_{2}$ & $\mathrm{~F}_{3}$ & $F_{i}$ & $F_{1}$ & $F_{2}$ & $E_{3}$ & $F_{4}$ & $F_{1}$ & $\mathrm{~F}_{2}$ & $\mathrm{~F}_{3}$ & $\mathrm{~F}_{4}$ \\
\hline MABDOEZ_... & 100 & 1 & 0 & 0 & 100 & 3 & 0 & 0 & 200 & 10 & 1 & 0 & 200 & 23 & 7 & 7 \\
\hline Auswoose ... & :-.: & i & 0 & 0 & $\ldots$ & $z$ & 0 & 0 & $=$ & 11 & ? & 1 & $\because \ldots$ & 33 & $\frac{21}{21}$ & 34 \\
\hline 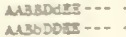 & .... & 1 & 0 & 0 & $\ldots$ & $i$ & 8 & 0 & -...- & 11 & 2 & 1 & $\ldots$ & 33 & 21 & $\frac{34}{34}$ \\
\hline LAs 3000 - -- & ---- & $i$ & 8 & 8 & $\ldots$ & 4 & 0 & 8 & $\cdots$ & 11 & 2 & 1 & $\ldots$ & 33 & 21 & 34 \\
\hline MUDDEe--- & ---- & 0 & 0 & 0 & $\ldots$ & 2 & 0 & 0 & -... & 3 & 1 & 1 & $\ldots$ & 12 & 16 & 40 \\
\hline Mussedre --. & -..- & 0 & 0 & 0 & $\ldots$ & 2 & 0 & 0 & --- & 3 & 1 & 1 & $\ldots$ & 12 & 16 & 40 \\
\hline AusbDOEZ --- & ...-- & 0 & 0 & 0 & $-\ldots$ & 2 & 0 & 0 & $\ldots$ & 3 & $i$ & i & -... & 12 & 16 & 40 \\
\hline CAM3DDES --- & $-\ldots$ & 0 & 0 & 0 & --- & 2 & 0 & 0 & $\ldots$ & 3 & 1 & 1 & --.- & 12 & 16 & 40 \\
\hline $\begin{array}{l}\text { Mass DCLe -.. } \\
\text { MabDoEe -.. }\end{array}$ & $\ldots$ & 2 & 0 & 0 & --- & 7 & 2 & 0 & $\ldots$ & 17 & 7 & 4 & $\ldots$ & 57 & 78 & 185 \\
\hline $\begin{array}{l}\text { Mabdote -.. } \\
\text { MasbDde3... }\end{array}$ & -... & 2 & 0 & 0 &.-- & 7 & 2 & 0 & $-\cdots$ & 17 & 7 & 4 & $\ldots$ & 57 & 78 & 185 \\
\hline 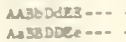 & $\ldots$ & 2 & 0 & 0 & $\ldots$ & 7 & 2 & 0 & -... & 17 & 7 & 4 & $\ldots$ & 57 & 78 & 285 \\
\hline $\begin{array}{l}\text { Ma 38DDCE -.- } \\
\text { Masadars -.- }\end{array}$ &.-- & 2 & 0 & 0 & $\ldots$ & 7 & 2 & 0 & $\ldots$ & 17 & 7 & 4 & --- & 57 & 78 & 185 \\
\hline MasaDdrs --- & --.- & 2 & 0 & 0 & $\ldots$ & 7 & 2 & 0 & $-\infty$ & 17 & 7 & 4 & -..- & 57 & 78 & 185 \\
\hline 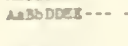 & $-\ldots$ & 2 & 0 & 0 & $-\cdots$ & 7 & 2 & 0 & .... & 17 & 7 & 4 & $\ldots$ & 57 & 78 & 185 \\
\hline Musdece... - & .... & 1 & 0 & 0 & $-\infty-$ & 3 & 2 & 1 & $\ldots$ & 6 & 6 & 5 & $-\ldots$ & 24 & 69 & 25 \\
\hline & $\ldots$ & 1 & 0 & 0 & $\ldots$ & 3 & 2 & 1 & $-\ldots$ & 6 & 6 & 5 & $\ldots$ & 24 & 69 & 25 \\
\hline AnsbDoce.... & $=-\infty$ & 1 & 0 & 0 & $\ldots$ & 3 & 2 & l & $\ldots$ & 6 & 6 & 5 & $\ldots$ & 24 & 69 & 25 \\
\hline Masb ddICE =. - & -..- & 2 & 0 & 0 & $\ldots$ & 3 & 2 & $i$ & .... & 6 & 6 & 5 & .... & 24 & 69 & 25 \\
\hline ASbDDEe -.. &..-- & 1 & 0 & 0 & $=--$ & 3 & 2 & i & $=-\infty$ & 6 & 6 & 5 & $\ldots$ & 24 & 69 & 25 \\
\hline MbobdaI .... & $\ldots$ & 1 & 0 & 0 & $\ldots$ & 3 & 2 & 1 & --.- & 6 & 6 & 5 & .... & 24 & 69 & 25 \\
\hline MS3DDee -.. - & -...- & 1 & 0 & 0 & $=--$ & 3 & 2 & i & $\ldots$ & 6 & 6 & 5 & $\ldots$ & 24 & 69 & 25 \\
\hline MusBddER -.. - & -... & 8 & 0 & 0 & ...- & 3 & 2 & 1 & $\ldots$ & 6 & 6 & 5 & $\ldots$ & 24 & 69 & 25 \\
\hline Aabboce -... & $\ldots$ & 1 & 0 & 0 & $-\ldots$ & 3 & 2 & $i$ & $\ldots$ & 6 & 6 & 5 & $\ldots$ & 24 & 69 & 25 \\
\hline Aa3sobte -... & $\ldots$ & 1 & 0 & 0 & $\ldots$ & 3 & 2 & 1 & --.. & 6 & 6 & 5 & $\ldots$ & 24 & 69 & 25 \\
\hline - 38 Dexz -.. & $-\ldots$ & 1 & 0 & 0 & $\ldots$ & 3 & 2 & 1 & $\ldots$ & 6 & 6 & 5 & $\ldots$ & 24 & 69 & 25 \\
\hline nasbotes... & -... & 1 & 0 & 0 & -... & 3 & 2 & $i$ & $-\ldots$ & 6 & 6 & 5 & ...- & 24 & 69 & 25 \\
\hline AMBDDCBE-..- & -...- & 4 & 1 & 0 & -.- & 13 & 9 & 4 & $\ldots$ & 29 & 29 & 24 & .... & 105 & 328 & 1,172 \\
\hline ABBDDdZe-.- & $\ldots$ & 4 & 1 & 0 & $-\infty-$ & 13 & 9 & 4 & $\ldots$ & 29 & 29 & 24 & $\ldots$ & 105 & 328 & 1,172 \\
\hline AMBSDDZe -.. & .... & - & 1 & 0 & ---- & 13 & 9 & 4 & .... & 29 & 29 & 24 & $\ldots$ & 105 & 328 & 1,172 \\
\hline MABSDET-... & $\ldots$ & - & 1 & 0 & =-- & 13 & 9 & 4 & -... & 29 & 29 & 24 & $\ldots$ & 105 & 328 & 1,172 \\
\hline Masddee... & .... & 0 & 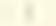 & 0 & .... & 2 & 2 & 2 & -... & 3 & 6 & 8 & $\ldots$ & 12 & 74 & 383 \\
\hline MLbDDee... & $\ldots$ & 0 & 3 & 0 & $\ldots$ & 2 & 2 & 2 & $\ldots$ & 3 & 6 & 8 & .... & 12 & 74 & 383 \\
\hline us bddzE ... & -... & 0 & 2 & 0 & $\ldots$ & 2 & 2 & 2 & --- & 3 & 6 & 8 & $\ldots$ & 12 & 74 & 383 \\
\hline aab $B D$ Dee -.. - & -... & 0 & 0 & 0 & .... & 2 & 2 & 2 & -... & 3 & 6 & 8 & -..- & 12 & 74 & 383 \\
\hline ABsddze -.. & - & 0 & 0 & 0 & -..- & 2 & 2 & 2 & $\ldots$ & 3 & 6 & 8 & $\ldots$ & 12 & 74 & 383 \\
\hline aAbbJ0E: -.. & - - & 0 & 0 & 0 & -..- & 2 & 2 & 2 & $\ldots$ & 3 & 6 & 8 & $\ldots$ & 12 & 74 & 383 \\
\hline MBDDdee -- - & --.-. & 2 & 1 & 0 & .... & 6 & 10 & 8 & .... & 12 & 27 & 38 & $\ldots$ & 48 & 330 & 1,778 \\
\hline Mabddze -.- &.-- & 2 & 1 & 0 & $\ldots$ & 6 & 10 & 8 & $\ldots$ & 12 & 27 & 38 & $\ldots$ & 48 & 330 & 1,778 \\
\hline MbbDdze ... & .... & 2 & 1 & 0 & $\ldots$ & 6 & 10 & 8 & $\ldots$ & 12 & 27 & 38 & $\ldots$ & 48 & 330 & 1,778 \\
\hline masdee... & $\ldots$ & 2 & 1 & 0 & $\ldots$ & 6 & 10 & 8 & $\ldots$ & 12 & 27 & 38 & $\ldots$ & 48 & 330 & 1,778 \\
\hline Masddze -.. & $\ldots$ & 2 & 1 & 0 & $\ldots$ & 6 & 10 & $B$ & -... & 12 & 27 & 38 & -... & 48 & 330 & 1,778 \\
\hline Aass DDee... & ...- & 2 & 1 & 0 & .... & 6 & 10 & 8 & $\ldots$ & 12 & 27 & 38 & $\ldots$ & 48 & 330 & 1,778 \\
\hline MBSddLEZ -.. & .... & 2 & 1 & 0 & .... & 6 & 10 & 8 & $\ldots$ & 12 & 27 & 38 & -... & 48 & 330 & 1,778 \\
\hline Masbodze -.. & -... & 2 & 1 & 0 & $\ldots$ & 6 & 10 & 8 & $\ldots$ & 12 & 27 & 38 & $\ldots$ & 48 & 330 & 1,778 \\
\hline ADbbDdRE-.. & -..- & 2 & 1 & 0 & $\ldots$ & 6 & 10 & 8 & $\ldots$ & 12 & 27 & 38 & $\ldots .$. & 48 & 330 & 1,778 \\
\hline assode -.. & $\ldots$ & 2 & 1 & 0 & .... & 6 & 10 & 8 & $\ldots$ & 12 & 27 & 38 & $\ldots$ & 48 & 330 & 1,778 \\
\hline aBSDDEe-.. & $\ldots$ & 2 & 1 & 0 & $\ldots$ & 6 & 10 & 8 & $\ldots$ & 12 & 27 & 38 & $\ldots$ & 48 & 330 & 1,778 \\
\hline a abbDdZ2 -.. & $\ldots$ & 2 & $i$ & 0 & $\ldots$ & 6 & 10 & 8 & -... & 12 & 27 & 38 & $\ldots$ & 48 & 330 & 1,778 \\
\hline Musbddee -.. - & $\ldots$ & 1 & 1 & 0 & $-\ldots$ & 3 & 13 & 20 & -... & 6 & 30 & 73 & -..- & 24 & 390 & 3,125 \\
\hline AbbDdee -..- & .... & $i$ & i & 0 & $\ldots$ & 3 & 13 & 20 & $\ldots$ & 6 & 30 & 73 & -... & 24 & 390 & 3,125 \\
\hline ubbdeze -.. & -... & 1 & 1 & 0 & $\ldots$ & 3 & 13 & 20 & $\ldots$ & 6 & 30 & 73 & -... & 24 & 390 & 3,125 \\
\hline nabiddee -.- & $\ldots$ & i & 1 & 0 & $\ldots$ & 3 & 13 & 20 & $\ldots$ & 6 & 30 & 73 & $-\ldots$ & 24 & 390 & 3,125 \\
\hline Abb DDee ...- & -.... & 1 & 1 & 0 & .... & 3 & 13 & 20 & .... & 6 & 30 & 73 & $\ldots$ & 24 & 390 & 3,125 \\
\hline Mabbddzz... & $\ldots$ & 1 & 1 & 0 & $\ldots$ & 3 & 13 & 20 & -..- & 6 & 30 & 73 & $\ldots$ & 24 & 390 & 3,125 \\
\hline a as Ddee -... & $=\ldots$ & 1 & 1 & 0 & $\ldots$ & 3 & 13 & 20 & ... & 6 & 30 & 73 & .... & 24 & 390 & 3,125 \\
\hline aBBddze -- & $\ldots$ & 1 & 1 & 0 & -..- & 3 & 13 & 20 & .... & 6 & 30 & 73 & $\ldots$ & 26 & 390 & 3,125 \\
\hline aABbDDee $=-$. & .... & $i$ & 1 & 0 & .... & 3 & 13 & 20 & .... & 6 & 30 & 73 & .... & 24 & 390 & 3,125 \\
\hline asBbddzz-.. & -..- & $i$ & i & 0 & .... & 3 & 13 & 20 & -... & 6 & 30 & 73 & -... & 24 & 390 & 3,125 \\
\hline asboDze... & --.- & i & 1 & 0 & .... & 3 & 13 & 20 & $=-\infty$ & 6 & 30 & 73 & -... & 24 & 390 & 3,125 \\
\hline aubbDCZZ -.. & $-\ldots$ & $i$ & 1 & 0 & $\ldots$ & 3 & 13 & 20 & $=-$ & 6 & 30 & 73 & $\ldots$. & 24 & 390 & 3,225 \\
\hline$\triangle a B b D d Z E--1$, & 930 & 228 & 26 & 3 & 3,960 & 707 & 185 & 75 & 3,960 & 93 & 375 & 286 & 7,920 & 2,870 & 2,907 & 9,675 \\
\hline Misbddee-. & - & 0 & 1 & 1 & -... & 2 & 18 & 55 & .... & 3 & 38 & 162 & .... & 12 & 512 & 6,382 \\
\hline asaddee.. & .... & 0 & $i$ & i & -..-. & 2 & 18 & s5 & .... & 3 & 38 & 162 & .... & 12 & 512 & 6,382 \\
\hline anboDee-- & $\ldots$ & 0 & $i$ & 1 & $\ldots$ & 2 & 18 & 55 & $\ldots$ & 3 & 38 & 162 & $\ldots$ & 12 & 512 & 6,382 \\
\hline abbddzE-- & $\ldots$ & 0 & 1 & $i$ & $\ldots$ & 2 & 18 & 55 & $\ldots$ & 3 & 38 & 162 &..- & 12 & 512 & 6,382 \\
\hline Aabsodee-.. & $\ldots$ & 126 & 60 & 9 & -... & 497 & 303 & 210 & -..- & 509 & 461 & 574 & $\ldots$ & 1,997 & 3,771 & 17,244 \\
\hline AaBbddRe-.. & $\ldots$ & 126 & 40 & 9 & $\ldots$ & 497 & 303 & 210 & $\ldots$ & 509 & 461 & 574 & $\ldots$ & 1,997 & 3,771 & 17,244 \\
\hline MabbDcze-.- & -... & 126 & 40 & 9 & $\ldots$ & 497 & 303 & 210 & .... & 509 & 461 & 574 & $\ldots$ & 1,997 & 3,771 & 17,244 \\
\hline saBbDCZe-.- & $\ldots$ & 126 & 40 & 9 & .... & 497 & 303 & 210 & $\ldots$ & 509 & 461 & 574 & $\cdots$ & 1,997 & 3,771 & 17,244 \\
\hline AsBbdee ... & .... & 124 & 86 & 39 & -... & 491 & 668 & 758 & -...- & 497 & 829 & 1,495 & $\ldots$ & 1,950 & 6,616 & 37,050 \\
\hline MsbbDdee... & .... & 124 & 86 & 39 & .... & 491 & 668 & 758 & -... & 497 & 829 & 1,495 & -.. & 1,950 & 6,616 & 37,050 \\
\hline AabbdaBe -.. - & $\ldots$ & 124 & 86 & 39 & $\ldots$ & 491 & 668 & 758 & $\ldots$ & 497 & 829 & 1,495 & -..- & 1,950 & 6,616 & 37,050 \\
\hline a 3bodee -.. & $\ldots$ & 126 & 86 & 39 & .... & 491 & 668 & 758 & $\ldots$ & 497 & 829 & 1,495 & $-\infty-$ & 1,950 & 6,616 & 37,050 \\
\hline sa3bddRe-.-- & $\ldots$ & 124 & 86 & 39 & .... & 491 & 668 & 758 & $\ldots$ & 497 & 829 & 1,495 & -... & 1,950 & 6,616 & 37,050 \\
\hline a abbDdZe-.. & -... & 124 & 86 & 39 & $\ldots$ & 491 & 668 & 758 & --- & 497 & 829 & 1,495 & $\ldots$ & 1,950 & 6,616 & 37,050 \\
\hline Aabbodee -.- & $-\ldots$ & 123 & 224 & 206 & -..- & 488 & 1,744 & 3,520 & .... & 491 & 1,924 & 5,216 & $\ldots$ & 1,926 & 14,686 & 99,178 \\
\hline aboddee... & .... & 123 & 224 & 206 & -... & 488 & 1,744 & 3,520 & $-\ldots$ & 491 & 1,924 & 5,216 & $\ldots$ & 1,926 & 14,686 & 99,178 \\
\hline asbb Ddee -... & .... & 123 & 224 & 206 & -... & 488 & 1,744 & 3,520 & -... & 491 & 1,924 & 5,216 & $\ldots$ & 1,926 & 14,686 & 99,178 \\
\hline aabbcdze... & $\ldots$ & 123 & 224 & 206 & $\ldots$ & 488 & 1,744 & 3,520 & $\cdots$ & 491 & 1,924 & 5,216 & $\ldots$. & 1,926 & 14,686 & 99,178 \\
\hline a abbddee ...- & $-\infty$ & 123 & 635 & 1,257 & $\ldots$ & 487 & 4,933 & 19,725 & $\ldots$ & 488 & 5,144 & 23,976 & $\ldots$ & 1,914 & 37,818 & 342,503 \\
\hline
\end{tabular}


MISC. PUB. 1178, U.S. DEPT. OF AGRICULTURE

Table 6.--Theoretical population and genotypic trends when native population of 2,000 insects (AABBDDEE) is overflooded by 198,000 or 396,000 partially sterile insects (aabbddee) during parental, $F_{1}, F_{2}$, and $F_{3}$ generations. Matings between partially sterile insects are assumed to yield no offspring--Continued

\begin{tabular}{|c|c|c|c|c|c|c|c|c|c|c|c|c|c|c|c|}
\hline Canatyon & & $\begin{array}{l}90 \mathrm{pe} \\
\text { five }\end{array}$ & $\begin{array}{l}\text { rcent, } \\
\text { fold }\end{array}$ & 96,000 & rele & $\begin{array}{l}80 \text { per } \\
\text { fivef }\end{array}$ & ent, & d ste & ity, & ${ }_{\text {te }}^{90 \mathrm{p}}$ & $\begin{array}{l}\text { rcent, } \\
\text { fold }\end{array}$ & ration & & $\begin{array}{l}80 \mathrm{pe} \\
\text { ten }\end{array}$ & $\begin{array}{l}\text { fcent, } \\
\text { fold }\end{array}$ \\
\hline & $\mathrm{F}_{1}$ & $\mathrm{~F}_{2}$ & $\mathrm{~F}_{3}$ & $F_{4}$ & $\mathrm{~F}_{1}$ & $\mathrm{~F}_{2}$ & $\mathrm{~F}_{3}$ & $\mathrm{~F}_{4}$ & $F_{1}$ & $\mathrm{~F}_{2}$ & $F_{3}$ & $\mathrm{~F}_{4}$ & $\mathrm{~F}_{1}$ & $\mathrm{~F}_{2}$ & $\mathrm{~F}_{3}$ \\
\hline AABBDDEE --- & 50 & 0 & 0 & 0 & 50 & 1 & 0 & 0 & 101 & 3 & 0 & 0 & 101 & 9 & 2 \\
\hline &.-- & 1 & 0 & 0 & --- & 2 & 0 & 0 & --- & 4 & 0 & 0 &..-- & 15 & 6 \\
\hline & $-\cdots$ & 1 & 0 & 0 & --- & 2 & 0 & 0 & -..- & 4 & 0 & 0 & -..- & 15 & 6 \\
\hline $\begin{array}{l}\text { AABbDDEE }=-- \\
\text { AaBBDDEE }---\end{array}$ & $\cdots$ & $\begin{array}{l}1 \\
1\end{array}$ & $\begin{array}{l}0 \\
0\end{array}$ & 0 & --- & 2 & $\begin{array}{l}0 \\
0\end{array}$ & 0 & $\cdots$ & 4 & 0 & 0 & --- & 15 & 6 \\
\hline AaBBDDEE --- & $\cdots$ & 1 & 0 & 0 & --- & 2 & 0 & 0 & $\cdots$ & 4 & 0 & 0 & --- & 15 & 6 \\
\hline $\begin{array}{l}\text { AABBDDee --- } \\
\text { AABBddEE --- }\end{array}$ & -..- & $\begin{array}{l}0 \\
0\end{array}$ & $\begin{array}{l}0 \\
0\end{array}$ & 0 & $--\cdot$ & 1 & 0 & 0 & $-\infty$ & 2 & 0 & 0 & $\ldots$ & 6 & 6 \\
\hline $\begin{array}{l}\text { AABBddEE --- } \\
\text { AAbb DDEE - .- }\end{array}$ & $-\cdots$ & $\begin{array}{l}0 \\
0\end{array}$ & $\begin{array}{l}0 \\
0\end{array}$ & $\begin{array}{l}0 \\
0\end{array}$ & $-\ldots$ & 1 & 0 & 0 & -.-- & 2 & 0 & 0 & $-\ldots-$ & 6 & 6 \\
\hline aa BBDDEE -..- & $\cdots$ & $\begin{array}{l}0 \\
0\end{array}$ & $\begin{array}{l}0 \\
0\end{array}$ & $\begin{array}{l}0 \\
0\end{array}$ & --- & $\begin{array}{l}1 \\
1\end{array}$ & $\begin{array}{l}0 \\
0\end{array}$ & $\begin{array}{l}0 \\
0\end{array}$ & -..- & 2 & 0 & 0 & $\ldots$ & 6 & 6 \\
\hline AABBDdEe -..- & -..- & 1 & 0 & 0 & --.- & 3 & 0 & & - & 2 & 0 & 0 & --- & 6 & 6 \\
\hline AABbDDEe - - & -..- & 1 & 0 & 0 & $-\cdots$ & $\begin{array}{l}3 \\
3\end{array}$ & 1 & 0 & $\ldots$ & 7 & 2 & 1 & --- & 27 & 26 \\
\hline AABbDdEE -.'- & -.-- & 1 & 0 & 0 & $\cdots$ & 3 & $\begin{array}{l}1 \\
1\end{array}$ & 0 & $\ldots$ & 7 & 2 & 1 & --- & 27 & 26 \\
\hline AaBBDDEe -.. & $\ldots$ & 1 & 0 & 0 & -.-- & 3 & $\begin{array}{l}1 \\
1\end{array}$ & 0 & 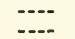 & 7 & 2 & 1 & --- & 27 & 26 \\
\hline AaBBDAEE -.- & -..- & 1 & 0 & 0 &.-- & 3 & $\begin{array}{l}1 \\
1\end{array}$ & 0 & $-\ldots$ & 7 & 2 & 1 & --- & 27 & 26 \\
\hline AaBbDDEE --_- & --- & 1 & 0 & 0 & $\cdots$ & 3 & $\begin{array}{l}1 \\
1\end{array}$ & 0 & 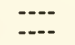 & $\begin{array}{l}7 \\
7\end{array}$ & 2 & 1 & $-\cdot-$ & $\begin{array}{l}27 \\
27\end{array}$ & $\begin{array}{l}26 \\
26\end{array}$ \\
\hline AABBDdee ---- & -... & 0 & 0 & 0 & ---- & 2 & & 0 & & 7 & 2 & & --.- & & \\
\hline AABBddEe -. & $\ldots$ & 0 & 0 & 0 & -- & 2 & $\begin{array}{l}1 \\
1\end{array}$ & 0 & & 3 & 2 & 1 & --- & 12 & 28 \\
\hline AABSDDee ... & -.-- & 0 & 0 & 0 & --- & 2 & $\begin{array}{l}1 \\
1\end{array}$ & $\begin{array}{l}0 \\
0\end{array}$ & --- & $\begin{array}{l}3 \\
3\end{array}$ & 2 & 1 & ---- & 12 & 28 \\
\hline AABbddEE _.-- & $\ldots$ & 0 & 0 & 0 & -.-- & 2 & $\begin{array}{l}1 \\
1\end{array}$ & $\begin{array}{l}0 \\
0\end{array}$ & --- & $\begin{array}{l}3 \\
3\end{array}$ & 2 & $\begin{array}{l}1 \\
1\end{array}$ & $-\cdots$ & $\begin{array}{l}12 \\
12\end{array}$ & $\begin{array}{l}28 \\
28\end{array}$ \\
\hline AAb $B O D E$-.- & --- & 0 & 0 & 0 & -..- & 2 & 1 & $\begin{array}{l}0 \\
0\end{array}$ &.-- & $\begin{array}{l}3 \\
3\end{array}$ & $\begin{array}{l}2 \\
2\end{array}$ & $\begin{array}{l}1 \\
1\end{array}$ & --- & $\begin{array}{l}12 \\
12\end{array}$ & $\begin{array}{l}28 \\
28\end{array}$ \\
\hline AAb DdEE -.- & $\ldots$ & 0 & 0 & 0 & 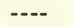 & 2 & 1 & 0 & $\ldots$ & 3 & 2 & 1 & $\ldots$ & 12 & 28 \\
\hline AaBBDDee .-- & --- & 0 & 0 & 0 & -.-- & 2 & 1 & 0 & -..- & 3 & 2 & 1 & ---- & 12 & 28 \\
\hline AaBBddEE --- & $\ldots$ & 0 & 0 & 0 & --- & 2 & 1 & 0 & $\ldots$ & 3 & 2 & 1 & ---- & 12 & 28 \\
\hline AabbDDEE -.- & $\ldots$ & 0 & 0 & 0 & ---- & 2 & 1 & 0 & --- & 3 & 2 & 1 & --- & 12 & 28 \\
\hline aaBBDDE --- & --- & 0 & 0 & 0 & --- & 2 & 1 & 0 & $\ldots$ & 3 & 2 & 1 & -... & 12 & 28 \\
\hline a BaBDdEE --- & $-\infty$ & 0 & 0 & 0 & --.- & 2 & 1 & 0 & $--\cdot$ & 3 & 2 & 1 &..- & 12 & 28 \\
\hline a aBbDDEE--- & --- & 0 & 0 & 0 & --- & 2 & & 0 & $\ldots$ & 3 & 2 & 1 & $\ldots$ & 12 & 28 \\
\hline AABbDdEe ... & -..- & 2 & 0 & 0 & ---- & 6 & 4 & 1 & ---- & 14 & 9 & 5 & --- & 51 & 125 \\
\hline $\mathrm{AaBBDdEe} . .$. & -... & 2 & 0 & 0 & --- & 6 & 4 & 1 & $-\ldots$ & 14 & 9 & 5 & --- & 51 & 125 \\
\hline $\mathrm{AaBbDDE}$ & --- & 2 & 0 & 0 & -..- & 6 & 4 & 1 & $-\ldots$ & 14 & s & 5 & $\ldots$ & 51 & 125 \\
\hline AaBbDdEE .-. & $\cdots$ & 2 & 0 & 0 & --- & 6 & 4 & 1 &.-- & 14 & 9 & 5 & 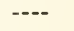 & 51 & 125 \\
\hline AABBddee ... & -..- & 0 & 0 & 0 & -...- & 1 & 1 & 1 & -..- & 2 & 2 & 2 & --- & 6 & 35 \\
\hline AAbbDDee ... & -- & 0 & 0 & 0 & --- & 1 & 1 & 1 & $\ldots$ & 2 & 2 & 2 & -..- & 6 & 35 \\
\hline AAbbd dEE _..- & $\ldots$ & 0 & 0 & 0 & --- & 1 & 1 & 1 & --.- & 2 & 2 & 2 &.-- & 6 & 35 \\
\hline a abBDDee ... & $\ldots$ & 0 & 0 & 0 & --- & 1 & 1 & 1 & --- & 2 & 2 & 2 & -... & 6 & 35 \\
\hline a aBBddEE & --- & 0 & 0 & 0 & $\ldots$ & 1 & 1 & 1 & -..- & 2 & 2 & 2 & $-\cdots$ & 6 & 35 \\
\hline aabbDDEE _.-- & $\ldots$ & 0 & 0 & 0 & $\cdots$ & 1 & 1 & 1 & --- & 2 & 2 & 2 & $\cdots$ & 6 & 35 \\
\hline AABbDdee_-_- & ---- & 1 & 0 & 0 &.--- & 3 & 5 & 3 & -..- & 6 & 11 & 9 & ...- & 25 & 148 \\
\hline $\mathrm{AABbddEe}=$ & $\ldots$ & 1 & 0 & 0 & ...- & 3 & 5 & 3 &.-- & 6 & 11 & 9 & $\ldots$ & 25 & 148 \\
\hline AAbBDdEe & $-\cdots$ & 1 & 0 & 0 & -... & 3 & 5 & 3 & $\cdots$ & 6 & 11 & 9 &.-- & 25 & 148 \\
\hline AaB BDdee ... & $\ldots$ & 1 & 0 & 0 & $\ldots-$ & 3 & 5 & 3 & --- & 6 & 11 & 9 & -- & 25 & 148 \\
\hline AaBBddEe & $\ldots$ & 1 & 0 & 0 & --- & 3 & 5 & 3 &.-- & 6 & 11 & 9 & -... & 25 & 148 \\
\hline AaBbDDee ... & --- & 1 & 0 & 0 & -..- & 3 & 5 & 3 & -- & 6 & 11 & 9 & $\ldots$ & 25 & 148 \\
\hline AABbddEE & ---- & 1 & 0 & 0 & $-\cdots$ & 3 & 5 & 3 & -..- & 6 & 11 & 9 & $\ldots$ & 25 & 148 \\
\hline AabbDDEe & --- & 1 & 0 & 0 & -... & 3 & 5 & 3 & -..- & 6 & 11 & 9 & -.-- & 25 & 148 \\
\hline AabbDdEE & $\ldots$ & 1 & 0 & 0 & $\ldots$ & 3 & 5 & 3 & -..- & 6 & 11 & 9 &.-- & 25 & 148 \\
\hline aaBBDAEe ... & $\ldots$ & 1 & 0 & 0 & -..- & 3 & 5 & 3 & $\cdots$ & 6 & 11 & 9 & --- & 25 & 148 \\
\hline a aBbDDEe ... & $\ldots$ & 1 & 0 & 0 & $-\cdots$ & 3 & 5 & 3 & -..- & 6 & 11 & 9 & $\ldots$ & 25 & 148 \\
\hline aaBbDdEE ... & $\ldots$ & 1 & 0 & 0 & --- & 3 & 5 & 3 & $-\ldots$ & 6 & 11 & 9 & -... & 25 & 148 \\
\hline AABbddee _.-- & --.-- & 0 & 0 & 0 &.--- & 2 & 6 & 8 & $\ldots$ & 3 & 14 & 23 & $\ldots$ & 12 & 194 \\
\hline AAB bDdee ... & $\ldots$ & 0 & 0 & 0 & --- & 2 & 6 & 8 & 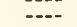 & 3 & 14 & 23 & - & 12 & 194 \\
\hline AAbbddEe -.. & $\cdots$ & 0 & 0 & 0 & $\cdots$ & 2 & 6 & 8 & --- & 3 & 14 & 23 & $\ldots$ & 12 & 194 \\
\hline AaBBddee --- & --- & 0 & 0 & 0 & ---- & 2 & 6 & 8 & --- & 3 & 14 & 23 &.--- & 12 & 194 \\
\hline AabbDDee--- & --- & 0 & 0 & 0 & --.- & 2 & 6 & 8 & -..- & 3 & 14 & 23 & ---- & 12 & 194 \\
\hline AabbddEE---- & --- & 0 & 0 & 0 & $-\cdots$ & 2 & 6 & 8 & --- & 3 & 14 & 23 & ---- & 12 & 194 \\
\hline aaBBDdee--- & -.-- & 0 & 0 & 0 & --- & 2 & 6 & 8 & $\cdots$ & 3 & 14 & 23 & --.-- & 12 & 194 \\
\hline a aBBddEe -- & -..- & 0 & 0 & 0 & $-\cdots$ & 2 & 6 & 8 & --- & 3 & 14 & 23 & --- & 12 & 194 \\
\hline a aBbDDee -.. & $\ldots$ & 0 & 0 & 0 & --- & 2 & 6 & 8 & --- & 3 & 14 & 23 & -..- & 12 & 194 \\
\hline aaBbddEE--- & --- & 0 & 0 & 0 & $--\cdot$ & 2 & 6 & 8 & --- & 3 & 14 & 23 & $--\cdot$ & 12 & 194 \\
\hline aabbDDEe--- & $\ldots$ & 0 & 0 & 0 & --- & 2 & 6 & 8 & ---- & 3 & 14 & 23 & --- & 12 & 194 \\
\hline a abb0dEE --- & $=-$ & 0 & 0 & 0 & $-\cdots$ & 2 & 6 & 8 & --- & 3 & 14 & 23 & --- & 12 & 194 \\
\hline AaBbDdEe--- & 1,990 & 177 & 16 & 1 & 3,980 & 605 & 119 & 33 & 3,980 & 717 & 193 & 84 & 7,960 & 2,445 & 1,591 \\
\hline AAb bddee--- & $\ldots$ & 0 & 1 & 0 & - & 1 & 9 & 25 &.-- & 2 & 19 & 62 & $\ldots$ & 6 & 272 \\
\hline aaBBddee--- & --.- & 0 & 1 & 0 & ---- & 1 & 9 & 25 & --- & 2 & 19 & 62 & -..- & 6 & 272 \\
\hline aabbDDee--- & $=--$ & 0 & 1 & 0 & ---- & 1 & 9 & 25 & -..- & 2 & 19 & 62 & ---- & 6 & 272 \\
\hline aabbddEE -.- & $=--$ & 0 & 1 & 0 & $\cdots$ & 1 & 9 & 25 & --- & 2 & 19 & 62 & --- & 6 & 272 \\
\hline AaBbDdee--- & -... & 125 & 31 & 6 & ...- & 499 & 244 & 121 & --.- & 505 & 315 & 231 & ---- & 200 & 2,630 \\
\hline AaBbddEe - -- & $\ldots$ & 125 & 31 & 6 & -..- & 499 & 244 & 121 & --.- & 505 & 315 & 231 & ---- & 200 & 2,630 \\
\hline AabbDdEe... & $-\ldots$ & 125 & 31 & 6 & -... & 499 & 244 & 121 & -.-- & 505 & 315 & 231 & --- & 200 & 2,630 \\
\hline aaBbDdEe-.- & --- & 125 & 31 & 6 & --- & 499 & 244 & 121 & $-\cdots$ & 505 & 315 & 231 & $--\cdot$ & 200 & 2,630 \\
\hline AaBbddee_-_- & -.-- & 125 & 78 & 31 & ---- & 496 & 616 & 557 & $\ldots$ & 499 & 693 & 830 & ---- & 1,975 & 5,618 \\
\hline AabbDdee... & $-\ldots$ & 125 & 78 & 31 & ---- & 496 & 616 & 557 & -... & 499 & 693 & 830 & --- & 1,975 & 5,618 \\
\hline AabbddEe.-- & $-\ldots$ & 125 & 78 & 31 & --- & 496 & 616 & 557 & $--\cdot$ & 499 & 693 & 830 & --.- & 1,975 & 5,618 \\
\hline aaBbDdee... & $\ldots$ & 125 & 78 & 31 & -..- & 496 & 616 & 557 & ---- & 499 & 693 & 830 & --- & 1,975 & 5,618 \\
\hline aaBbddEe-.. & -... & 125 & 78 & 31 & ---- & 496 & 616 & 557 & --- & 499 & 693 & 830 & --- & 1,975 & 5,618 \\
\hline aabbDdEe... & --- & 125 & 78 & 31 & --- & 496 & 616 & 557 & $\ldots$ & 499 & 693 & 830 & -... & 1,975 & 5,618 \\
\hline Aabbddee---- &.--- & 124 & 217 & 186 & --- & 494 & 1,718 & 3,097 & .... & 496 & 1,809 & 3,831 & -..- & 1,963 & 14,190 \\
\hline aabbddee.-- &.-- & 124 & 217 & 186 & -... & 494 & 1,718 & 3,097 & $\ldots$ & 496 & 1,809 & 3,831 & -..- & 1,963 & 14,190 \\
\hline aabbDdee.-- & --- & 124 & 217 & 186 & -... & 494 & 1,718 & 3,097 & -..- & 496 & 1,809 & 3,831 & -..- & 1,963 & 14,190 \\
\hline aabbddEe--ᄀ- & $\ldots$ & 124 & 217 & 186 & --- & 494 & 1,718 & 3,097 & --- & 496 & 1,809 & 3,831 & --- & 1,963 & 14,190 \\
\hline aabbddee-.- & $---\infty$ & 124 & 634 & 1,214 & ---- & 493 & 5,000 & 19,293 & ---- & 494. & 5,112 & 21,379 & ---- & 1,956 & 39,218 \\
\hline & 2,040 & 2,082 & 2,126 & 2,171 & 4,030 & 8,182 & 16,861 & 35,788 & 4,080 & 8,494 & 18,414 & 43,374 & 8,060 & 33,206 & 148,238 \\
\hline
\end{tabular}


Table -.-Teoretical population and genotypic trends of male insects when native population of 2,000 Insects (3 autosomal genes B8DDE. olus : sex-11nked gebe $\mathrm{M}$; iesle is heterogmetic) 1 s overflooded by 198,000 or 396,000 partially sterile insects (aabbddee and a-bbddee) during perental, $F_{1}, F_{2}$, and $F_{3}$ generations. Matings between partially sterile insects are assumed to yleld no of fspring

\begin{tabular}{|c|c|c|c|c|c|c|c|c|c|c|c|c|c|}
\hline & & & & 198 & 0 reled & sed vit & $h$ ind $1 c$ & cated st & 1109,10 & resse, & and ger & neration & \\
\hline cenctrse & & $\begin{array}{l}\text { So pe } \\
\text { fIve }\end{array}$ & $\begin{array}{l}\text { cent, } \\
\text { old }\end{array}$ & & & $\begin{array}{l}\text { So per } \\
\text { fiver }\end{array}$ & $\begin{array}{l}\text { cent, } \\
\text { old }\end{array}$ & & & $\begin{array}{l}0 \text { pere } \\
\text { tenfo }\end{array}$ & ent, & & \\
\hline & $\bar{F}_{1}$ & $\varepsilon_{2}$ & $\mathrm{E}_{3}$ & $F_{6}$ & $F_{1}$ & $\mathrm{~F}_{2}$ & $\mathrm{~F}_{3}$ & $F_{-}$ & $F_{1}$ & $F_{2}$ & $\mathrm{~F}_{3}$ & $F_{4}$ & $E_{1}$ \\
\hline MASADOES -..- & so & 1 & 0 & 0 & so & 1 & 0 & 0 & 100 & 5 & 0 & 0 & 100 \\
\hline MUIDDEe ... & -..- & 1 & 0 & 0 & $-\ldots$ & 2 & 0 & 0 & $-\ldots$ & 5 & 1 & 0 & $\cdots$ \\
\hline MS3DdEE -.- & $\ldots$ & 1 & 0 & 0 & $\cdots$ & 2 & 0 & 0 & --- & 5 & 1 & 0 & $\ldots$ \\
\hline M3ODOES -.. & $-\ldots$ & 1 & 0 & 0 & $-\ldots$ & 2 & 0 & 0 & $\ldots$ & 5 & 1 & 0 & $-\ldots$ \\
\hline Aassidoen -... & $\ldots$ & 1 & 0 & 0 & $\ldots$ & 2 & 0 & 0 & -... & 5 & 1 & 0 & --- \\
\hline MusD Dee -..- & $-\ldots$ & 0 & 0 & 0 &.--- & 1 & D & 0 & --- & 2 & 1 & 0 & --- \\
\hline Musdess -.- & $\cdots$ & 0 & 0 & 0 & $\cdots$ & 1 & 0 & 0 & ---- & 2 & 1 & 0 & $\ldots$ \\
\hline MS BDDEE -.- & $\ldots$ & 0 & 0 & 0 & ---- & 1 & 0 & 0 & $\ldots$ & 2 & 1 & 0 & $\cdots$ \\
\hline uns spDes -.. & $\ldots$ & 0 & 0 & 0 & --- & 1 & 0 & 0 & $\ldots$ & 2 & 1 & 0 & $-\cdots$ \\
\hline Mas DedEe -. - & $-\infty-$ & 1 & 0 & 0 & $\ldots$ & 4 & 1 & 0 & ---- & 9 & 4 & 2 & -..- \\
\hline MUSDDEe -.. & 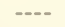 & 1 & 0 & 0 & $\ldots$ & 4 & 1 & 0 & --- & 9 & 4 & 2 & --- \\
\hline MSBDdER -.. & --- & 1 & 0 & 0 & -..- & 4 & 1 & 0 & -.-- & 9 & 4 & 2 & -...- \\
\hline Maspose -.- & -... & 1 & 0 & 0 & --- & 6 & 1 & 0 & $-\ldots$ & 9 & 4 & 2 & $\ldots$ \\
\hline AusBDdRe ... & -.- & 1 & 0 & 0 & --- & 4 & 1 & 0 & $\ldots$ & 9 & 4 & 2 & ---- \\
\hline Aa,3b DOEZ -.. & --- & 1 & 0 & 0 & $-\infty-$ & 4 & 1 & 0 & $\ldots$ & 9 & 4 & 2 & -..- \\
\hline MaBdee -- & --- & 0 & 0 & 0 & $-\infty$ & 2 & 1 & 0 & $-\ldots$ & 3 & 3 & 2 & ---- \\
\hline MusddBe -.. & ---- & 0 & 0 & 0 & -... & 2 & 1 & 0 & -.-- & 3 & 3 & 2 & $\ldots$ \\
\hline Mabocee -.. & --.- & 0 & 0 & 0 & $\ldots$ & 2 & 1 & 0 & ---- & 3 & 3 & 2 & -..-- \\
\hline MabddEe =. & $\cdots$ & 0 & 0 & 0 & --- & 2 & 1 & 0 & --- & 3 & 3 & 2 & $\ldots$ \\
\hline MabDes =-- & -- - & 0 & 0 & 0 & --- & 2 & 1 & 0 & $-\ldots$ & 3 & 3 & 2 & $\ldots$ \\
\hline AusDDdEe -... & -.- & 0 & 0 & 0 & --- & 2 & 1 & 0 & -... & 3 & 3 & 2 &.-- \\
\hline AasadDee --- & --- & 0 & 0 & 0 & $-\infty$ & 2 & 1 & 0 & $\ldots$ & 3 & 3 & 2 & $\ldots$ \\
\hline AusaddEE -..- & $\ldots$ & 0 & 0 & 0 &.--- & 2 & 1 & 0 & -..- & 3 & 3 & 2 & ---- \\
\hline MabbDDEE --- & ---- & 0 & 0 & 0 & $\ldots$ & 2 & 1 & 0 & ---- & 3 & 3 & 2 & ---- \\
\hline antabole -.- & -..- & 0 & 0 & 0 & $\ldots$ & 2 & 1 & 0 & --.- & 3 & 3 & 2 & ---- \\
\hline asBDdrz .... & $\ldots$ & 0 & 0 & 0 & -... & 2 & 1 & 0 & -... & 3 & 3 & 2 & $\cdots$ \\
\hline ans bDoes =. - &.--- & 0 & 0 & 0 & ---- & 2 & 1 & 0 & ---- & 3 & 3 & 2 & $\cdots$ \\
\hline Masbde =-. & - - - & 2 & 0 & 0 &..-- & 7 & 4 & 2 & -... & is & 14 & 12 & $-\ldots$ \\
\hline AnsaJdee --- & $-\cdots$ & 2 & 0 & 0 & $\ldots$ & 7 & 4 & 2 & -...- & 15 & 14 & 12 & $\ldots$ \\
\hline AEBbDORe $=-$ & -..- & 2 & 0 & 0 & $-\ldots$ & 7 & 4 & 2 & --.- & 15 & 14 & 12 & ---- \\
\hline As 30 DedR ... & $\ldots$ & 2 & 0 & 0 & $-\ldots$ & 7 & 4 & 2 & -.-- & 15 & 14 & 12 & $-\cdots$ \\
\hline Masd de e -.. & -..- & 0 & 0 & 0 & $\ldots$ & 1 & 1 & 1 & --.-- & 2 & 3 & 4 & -... \\
\hline MubbDee -.. & ---- & 0 & 0 & 0 & -... & 1 & 1 & 1 & --- & 2 & 3 & 4 & ---- \\
\hline MbbddEL ... & --- & 0 & 0 & 0 & --- & 1 & 1 & 1 & $\ldots$ & 2 & 3 & 4 &.-- \\
\hline aAbBDDee -..- & ---- & 0 & 0 & 0 & --- & 1 & 1 & 1 & ---- & 2 & 3 & 4 & $\ldots$ \\
\hline asisda -.-- & ---- & 0 & 0 & 0 & --.- & 1 & 1 & 1 & $\ldots$ & 2 & 3 & 4 & -... \\
\hline asbsoez -.. & 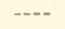 & 0 & 0 & 0 & $\ldots$ & 1 & 1 & 1 & $-\cdots$ & 2 & 3 & 4 & --- \\
\hline Nabdee -..- & -..- & 1 & 0 & 0 & -..- & 3 & 5 & 4 & -... & 6 & 13 & 19 & --.- \\
\hline Mabddre =-- & $\ldots$ & 1 & 0 & 0 & -.- & 3 & 5 & 4 &.-- & 6 & 13 & 19 & -..-- \\
\hline MSBDCEe -.. & -..- & 1 & 0 & 0 & $\ldots$ & 3 & 5 & 4 & -... & 6 & 13 & 19 & -..- \\
\hline MaBs Ddee -... & ... & 1 & 0 & 0 & $-\ldots$ & 3 & 5 & 4 & --.-- & 6 & 13 & 19 & -... \\
\hline AasiddBe -.- & $-\cdots$ & 1 & 0 & 0 & $-\ldots$ & 3 & 5 & 4 & $\ldots$ & 6 & 13 & 19 & -...- \\
\hline AasbDDee -.- & -.-- & 1 & 0 & 0 & $\ldots$ & 3 & 5 & 4 & --.- & 6 & 13 & 19 & --- \\
\hline Mibddzz ... & $-\ldots$ & 1 & 0 & 0 & -..- & 3 & 5 & 4 & -... & 6 & 13 & 19 & -... \\
\hline MBDDDEe =.- & -..- & 1 & 0 & 0 & .... & 3 & 5 & 4 & $\cdots$ & 6 & 13 & 19 & --- \\
\hline ASBbDAEZ -.- & --- & 1 & 0 & 0 & $-\cdots$ & 3 & 5 & 4 & ---- & 6 & 13 & 19 & $\ldots$ \\
\hline ABBDdBe -.- & $\ldots$ & 1 & 0 & 0 & $\cdots$ & 3 & 5 & 4 & --. & 6 & 13 & 19 & $-\cdots$ \\
\hline ansb DORe --- & ---- & 1 & 0 & 0 & $\ldots$ & 3 & 5 & 4 & --- & 6 & 13 & 19 & --.- \\
\hline asbDded -.. & $\cdots$ & 1 & 0 & 0 & -..- & 3 & 5 & 4 & $\ldots$ & 6 & 13 & 19 & 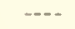 \\
\hline Masbdece... & --- & 0 & 0 & 0 & $-\ldots$ & 2 & 6 & 10 & -... & 3 & 15 & 36 & $\ldots$ \\
\hline Mbidice...- & --.- & 0 & 0 & 0 & -..- & 2 & 6 & 10 & -... & 3 & 15 & 36 & .... \\
\hline Mbbddze... & - & 0 & 0 & 0 & $\ldots$ & 2 & 6 & 10 & -... & 3 & 15 & 36 & -... \\
\hline As3Bddee.... & -..- & 0 & 0 & 0 & --- & 2 & 6 & 10 & -..- & & 15 & 36 & -..- \\
\hline AabbDjee... & -- & 0 & 0 & 0 &.-- & 2 & 6 & 10 & $\ldots$ & 3 & 15 & 36 & 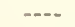 \\
\hline AubbddEZ -.. & ---- & 0 & 0 & 0 & 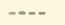 & 2 & 6 & 10 & --- & 3 & 15 & 36 &.-- \\
\hline anBudee--- & $--\infty$ & 0 & 0 & 0 & $\ldots$ & 2 & 6 & 10 &.--- & 3 & 15 & 36 &.--- \\
\hline a3BddZe--- & --- & 0 & 0 & 0 & $-\cdots$ & 2 & 6 & 10 & --- & 3 & 15 & 36 & --- \\
\hline aabbDCec--- & -..- & 0 & 0 & 0 & --- & 2 & 6 & 10 & $\ldots$ & 3 & 15 & 36 & $\ldots$ \\
\hline aลbb ddzZ -.. & -... & 0 & 0 & 0 & $\cdots$ & 2 & 6 & 10 & $-\ldots$ & 3 & 15 & 36 & .... \\
\hline asbDoze-.. & $\ldots$ & 0 & 0 & 0 & $\ldots$ & 2 & 6 & 10 & $-\cdots$ & 3 & 15 & 36 & -... \\
\hline aubbDezE =- - & 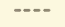 & 0 & 0 & 0 & $\ldots$ & 2 & 6 & 10 & ---- & 3 & 15 & 36 & $-\ldots$ \\
\hline Aasb DdRe.-. 9 & 990 & 116 & 13 & 2 & 1,980 & 353 & 92 & 38 & 1,980 & 465 & 188 & $14 \%$ & 3,960 \\
\hline Mbbddee -.. & --- & 0 & 1 & 0 & $-\ldots$ & 1 & 9 & 28 &.-- & 2 & 19 & 81 & $-\ldots$ \\
\hline saBbddee --- & ---- & 0 & 1 & 0 & --- & 1 & 9 & 28 & --- & 2 & 19 & 81 & -..- \\
\hline eabbliee -.. & $\ldots$ & 0 & 1 & 0 & $\cdots$ & 1 & 9 & 28 & $\cdots$ & 2 & 19 & 81 & $\cdots$ \\
\hline abbiddzz -.. & $\ldots$ & 0 & 1 & 0 & $\ldots$ & 1 & 9 & 28 & -..- & 2 & 19 & 81 & $-\cdots$ \\
\hline MabDdee -..- & -... & 63 & 20 & 5 & -... & 249 & 151 & 105 & -... & 254 & 231 & 289 & $-\ldots$ \\
\hline AaBbddele... & $\ldots$ & 63 & 20 & s & $\cdots$ & 249 & 151 & 105 & $-\ldots$ & 254 & 231 & 289 & ---- \\
\hline AsלbüdBe-.- & --- & 63 & 20 & s & --- & 249 & 151 & 105 & ---- & 254 & 231 & 289 & ---- \\
\hline aaßb D̃dze --- & --- & 63 & 20 & 5 & --- & 249 & 151 & 105 & ---- & 254 & 231 & 289 & -..- \\
\hline AaBbddee-.. &.-+- & 52 & 43 & 19 & $\ldots$ & 246 & 334 & 381 & --.- & 248 & 414 & 755 & -... \\
\hline Aabs Deee -.. & $-\cdots$ & 52 & 43 & 19 & $\ldots$ & 245 & 334 & 381 & $\cdots$ & 248 & 414 & 755 & $\ldots$ \\
\hline Aabbddze-.. & $-\ldots$ & 62 & 43 & 19 & $\cdots$ & 246 & 336 & 381 & $-\infty$ & 248 & 414 & 755 &.-- \\
\hline aazbDcee --- & --- & 62 & 43 & 19 & $-\cdots$ & 246 & 334 & 381 & --- & 248 & 414 & 755 & --- \\
\hline aABbddze --- & --- & 62 & 43 & 19 & $-\cdots$ & 246 & 334 & 381 & --- & 248 & 414 & 755 &.--- \\
\hline a.abbidze --- & --- & 62 & 43 & 19 & --- & 246 & 334 & 381 & $\cdots$ & 248 & 414 & 755 & --- \\
\hline Aaboddee... & $\ldots$ & 62 & 112 & 103 & 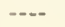 & 264 & 872 & 1,773 & 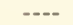 & 245 & 962 & 2,643 & $\ldots$ \\
\hline aaB bcdee--. & --- & 62 & 112 & 103 &.-- & 244 & 872 & 1,773 & $\cdots$ & 245 & 962 & 2,643 & --.- \\
\hline a ab bDdee --. & $-\infty-$ & 62 & 112 & 103 & ---- & 244 & 872 & 1,773 & ---- & 245 & 962 & 2,643 & $\ldots-$ \\
\hline aabboddze -.. & --- & 62 & 112 & 103 &.-- & 246 & 872 & 1,773 & -..- & 245 & 962 & 2,643 & $\cdots$ \\
\hline asbbddee --- & $\cdots$ & 61 & 348 & 832 & --- & 243 & 2,700 & 12,847 & $\cdots$ & 244 & 281 & 15,140 & -..- \\
\hline oral-... 1 , & 0601, & 983 & 1,160 & 1,386 & 2,030 & 4,182 & 9,1072 & 22,987 & 2,080 & 4,502 & 0,805 & 32,644 & 4,0601 \\
\hline
\end{tabular}


Table 7.--Theoretical population and genotypic trends of male insects when native population of 2,000 insects (3 autosomal genes B8DDEE plus 1

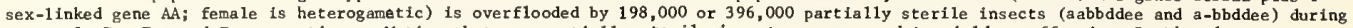
parental, $F_{1}, F_{2}$, and $F_{3}$ generations. Matings between partially sterile insects are assumed to yield no offspring-Continued

\begin{tabular}{|c|c|c|c|c|c|c|c|c|c|c|c|c|c|}
\hline & & & & 396 , & released & with i & dicated & sterilit & Increase, & and ge & ration & & \\
\hline Genotype & & $\begin{array}{r}90 \mathrm{p} \\
\mathrm{fiv}\end{array}$ & $\begin{array}{l}\text { cent, } \\
\text { old }\end{array}$ & & & $\begin{array}{l}8 \mathrm{D} \text { pe } \\
\text { five }\end{array}$ & $\begin{array}{l}\text { cent, } \\
\text { old }\end{array}$ & & & ${ }^{90}$ & $\begin{array}{l}\text { rcent, } \\
\text { fold }\end{array}$ & & \\
\hline & $F_{1}$ & $F_{2}$ & $E_{3}$ & $\mathrm{~F}_{4}$ & $F_{1}$ & $F_{2}$ & $E_{3}$ & $F_{4}$ & $F_{1}$ & $F_{2}$ & $E_{3}$ & $E_{4}$ & $F_{1}$ \\
\hline AABBDDEE_-.- & 25 & 0 & 0 & 0 & 25 & 1 & 0 & 0 & 50 & 2 & 0 & 0 & 50 \\
\hline AABBDDEe..-- & -.- & 0 & 0 & 0 & $-\cdots-$ & $\mathbf{1}$ & 0 & 0 & $\cdots$ & 2 & 0 & 0 &.-- \\
\hline AABBDdEE--- & --- & 0 & 0 & 0 & -2 & 1 & 0 & 0 & $m$ & 2 & 0 & 0 & --- \\
\hline AABbDDEE $=-$ & $\ldots$ & 0 & 0 & 0 & -ne & 1 & 0 & 0 & $-\infty$ & 2 & 0 & 0 & -- \\
\hline AaBBDDEE --- & $\cdots$ & 0 & 0 & 0 & --- & 1 & 0 & 0 & --- & 2 & 0 & 0 & $-\infty$ \\
\hline AABBDDee =-- & $\ldots$ & 0 & 0 & 0 & -...- & 0 & 0 & 0 & -... & 1 & 0 & 0 & ---- \\
\hline AABBddEE--- & --- & 0 & 0 & 0 & $\ldots$ & 0 & 0 & 0 & $-\cdots$ & 1 & 0 & 0 & $\ldots$ \\
\hline AAbbDDEE--- & --- & 0 & 0 & 0 & --- & 0 & 0 & 0 & ...- & 1 & 0 & 0 & $-\infty$ \\
\hline aABBDDEE--- & $-\cdots$ & 0 & D & 0 & $\cdots$ & 0 & 0 & 0 & --- & 1 & 0 & 0 & $-\cdots$ \\
\hline AABBDdEe--- & $m$ & 0 & 0 & 0 & ---- & 2 & 0 & 0 & ---- & 4 & 1 & 0 & --- \\
\hline AABBbDDEe--- & $\cdots$ & 0 & 0 & 0 & $\cdots$ & 2 & 0 & 0 & $m--$ & 4 & 1 & 0 & -- \\
\hline AABbDdEE--- & $-\cdots$ & 0 & 0 & 0 & -..- & 2 & 0 & 0 & $\ldots$ & 4 & 1 & 0 & --- \\
\hline Aa8BDDEe--- & --- & 0 & 0 & 0 & $-\cdots$ & 2 & 0 & 0 & --- & 4 & 1 & 0 & $\ldots$ \\
\hline AaBBDdEE--- & $\cdots$ & 0 & 0 & 0 & $\cdots$ & 2 & 0 & 0 & $\cdots$ & 4 & 1 & 0 & $\cdots$ \\
\hline AaBbDDEE--- & $\cdots$ & 0 & 0 & 0 & $\cdots$ & 2 & 0 & 0 & $-\cdots$ & 4 & 1 & 0 & --- \\
\hline AABBDdee--- & $\ldots$ & 0 & 0 & 0 & $\ldots$ & 1 & 0 & 0 & $\ldots-$ & 2 & 1 & 0 & $\ldots$ \\
\hline AABBddEe- -- & $m-$ & 0 & 0 & 0 & $\cdots$ & 1 & 0 & 0 & --- & 2 & 1 & 0 & --- \\
\hline AABbDDee--- & $\cdots$ & 0 & 8 & 0 & $-\ldots$ & 1 & 0 & 0 & --- & 2 & 1 & 0 & --- \\
\hline AABbddEE--- & --- & 0 & $\begin{array}{l}0 \\
0\end{array}$ & 0 & --- & 1 & 0 & 0 & 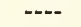 & 2 & 1 & 0 & ---- \\
\hline AAbbDDEe---- & $\cdots$ & 0 & $\begin{array}{l}0 \\
0\end{array}$ & D & --.- & 1 & 0 & 0 & $-\ldots$ & 2 & 1 & 0 & --.- \\
\hline AAbbDdEE--- & ---- & 0 & $\begin{array}{l}0 \\
0\end{array}$ & 0 & $-\infty$ & 1 & 0 & 0 & $\cdots$ & 2 & 1 & 0 & $\cdots$ \\
\hline AaBBDDee--- & $\cdots$ & 0 & $\begin{array}{l}0 \\
0\end{array}$ & 0 & -... & 1 & 0 & 0 & $\cdots$ & 2 & 1 & 0 & $\cdots$ \\
\hline AABBddEE--- & $\cdots$ & 0 & 0 & 0 & $\cdots$ & 1 & 0 & 0 & --- & 2 & 1 & 0 & $\cdots$ \\
\hline AabbDDEE-.- & $-\cdots$ & 0 & 0 & 0 & $\cdots$ & 1 & 0 & 0 & ---- & 2 & 1 & 0 & --- \\
\hline agBBDDEe--- & --- & 0 & 0 & 0 & --- & 1 & 0 & 0 & --- & 2 & 1 & 0 & --- \\
\hline aaBBDdEE--- & --- & 0 & 0 & 0 & ---- & 1 & 0 & 0 & --- & 2 & 1 & 0 & $\cdots$ \\
\hline aABbDDEE--- & --- & 0 & 0 & 0 & --- & 1 & 0 & 0 & --- & 2 & 1 & 0 & $\cdots$ \\
\hline AABbDdEe- - & $\cdots$ & 1 & 0 & 0 & .... & 3 & 2 & 1 & --- & 7 & 5 & 2 &.-- \\
\hline AaBBDdEe- - - & -- & 1 & 0 & 0 &..- & 3 & 2 & 1 & 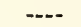 & 7 & 5 & 2 & --- \\
\hline $\mathrm{Aa} \mathrm{Bb} D D E e---$ & $\ldots$ & 1 & 0 & 0 & -.- & 3 & 2 & 1 &.-- & 7 & 5 & 2 & --- \\
\hline AaBbDdEE-- - & $-\cdots$ & 1 & 0 & 0 & -..- & 3 & 2 & 1 & --- & 7 & 5 & 2 & $\cdots$ \\
\hline AABBddee--- & --- & 0 & 0 & 0 & $\ldots$ & 0 & 1 & 0 & .... & 1 & 1 & 1 & -.- \\
\hline AAbbDDee--- & $\ldots$ & 0 & 0 & 0 & -..- & 0 & 1 & 0 & --- & 1 & 1 & 1 & --- \\
\hline AAbbddEE--- & -..- & 0 & 0 & 0 & 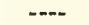 & 0 & 1 & 0 & --- & 1 & 1 & 1 & ---- \\
\hline asbBDDee-- & $\ldots$ & 0 & 0 & 0 & --- & 0 & 1 & 0 & -..- & 1 & 1 & 1 & --.- \\
\hline a aBBddEE-.. & $\ldots$ & 0 & 0 & 0 & --- & 0 & 1 & 0 & $-\cdots$ & 1 & 1 & 1 & $\cdots$ \\
\hline aabbDDEE=-- & $-\cdots$ & 0 & 0 & 0 & $-\cdots$ & 0 & 1 & 0 & $\cdots$ & 1 & $\mathbf{1}$ & 1 & $\cdots$ \\
\hline AABbDdee--- & --- & 0 & 0 & 0 & --- & 2 & 2 & 1 & -..- & 3 & 5 & 5 & -... \\
\hline AABbddEe- & ---- & 0 & 0 & 0 & --- & 2 & 2 & 1 & $\ldots$ & 3 & 5 & 5 & $\ldots$ \\
\hline AAbbDdEe-.-- & -... & 0 & 0 & 0 & -... & 2 & 2 & 1 & -..- & 3 & 5 & 5 &.-- \\
\hline AsBBDdee-.- & $-\ldots$ & 0 & 0 & 0 & -..- & 2 & 2 & 1 & -..- & 3 & 5 & 5 & $\cdots$ \\
\hline AaBBddEe-.- &.--- & 0 & 0 & 0 & --- & 2 & 2 & 1 & $\ldots$ & 3 & 5 & 5 & --- \\
\hline AaBbDDee_--- & --.- & 0 & 0 & 0 & -.- & 2 & 2 & 1 & --. & 3 & 5 & 5 & --- \\
\hline AABbddEE & $\ldots$ & 0 & 0 & 0 & ---- & 2 & 2 & 1 & $-\ldots$ & 3 & 5 & 5 & -..- \\
\hline AsbbDDEe $_{7}--$ & --.- & 0 & 0 & 0 &.-- & 2 & 2 & 1 & -... & 3 & 5 & 5 & --- \\
\hline AabbDdEE & --- & 0 & 0 & 0 & --- & 2 & 2 & 1 & -... & 3 & 5 & 5 &.-- \\
\hline AaBBDdEe _.. & --- & 0 & 0 & 0 & -..- & 2 & 2 & 1 & -.-- & 3 & 5 & 5 & --- \\
\hline QQBBDDDEe_-_- & $\ldots$ & 0 & 0 & 0 & -..- & 2 & 2 & 1 & $\ldots$ & 3 & 5 & 5 & $\cdots$ \\
\hline$\triangle a B b D d E E_{\alpha} \ldots$ & -... & 0 & 0 & 0 & --- & 2 & 2 & 1 & --- & 3 & 5 & 6 & --- \\
\hline AABbddee...- & -..- & 0 & 0 & 0 & ...- & 1 & 3 & 4 & .... & 2 & 7 & 11 & ---- \\
\hline AAbbDdee +- & $\ldots$ & 0 & 0 & 0 & $\cdots$ & 1 & 3 & 4 & $\ldots$ & 2 & 7 & 11 &.-- \\
\hline AAbbddEe_.-- & -.- & D & 0 & 0 & $\ldots$ & 1 & 3 & 4 &..- & 2 & 7 & 11 & --- \\
\hline $\mathrm{AaBBddee}$ & --- & 0 & 0 & 0 & $\ldots$ & 1 & 3 & 4 & -.. & 2 & 7 & 11 & -..- \\
\hline AabbDDee.... & -... & 0 & 0 & 0 &.-- & 1 & 3 & 4 &.-- & 2 & 7 & 11 & -- \\
\hline AabbddEE & --.- & 0 & 0 & 0 & --- & 1 & 3 & 4 & $\ldots$ & 2 & 7 & 11 & -..- \\
\hline aQBBDdee $\rightarrow--$ & -... & 0 & 0 & 0 &.-- & 1 & 3 & 4 & -... & 2 & 7 & 11 &.-- \\
\hline a aBBddEe... & ---- & 0 & 0 & 0 &..- & 1 & 3 & 4 & $\ldots$ & 2 & 7 & 11 & --- \\
\hline asBbDDee-_-- & -..- & 0 & 0 & 0 &.-- & 1 & 3 & 4 & $\ldots$ & 2 & 7 & 11 & $\ldots$ \\
\hline a BBbddEE_. - & $\ldots$ & 0 & 0 & 0 & $\cdots$ & 1 & 3 & 4 & $\ldots$ & 2 & 7 & 11 & -- \\
\hline aabbDDEe_..- & -..- & 0 & 0 & 0 & --- & 1 & 3 & 4 & $-\cdots$ & 2 & 7 & 11 & $\cdots$ \\
\hline aabbDdEE--- & --- & 0 & 0 & 0 & $\cdots$ & 1 & 3 & 4 & $-\ldots$ & 2 & 7 & 11 & $-\cdots$ \\
\hline AaBbDdEe-... & 995 & 88 & 8 & 1 & 1,990 & 302 & 59 & 16 & $1,99 \mathrm{D}$ & 359 & 96 & 42 & 3,980 \\
\hline AAbbddee--- &.-- & 0 & 0 & 0 & -..-- & 0 & 4 & 13 & --.- & 1 & 9 & 31 & -.. \\
\hline aaBBddee--. &.-- & 0 & 0 & 0 & --- & 0 & 4 & 13 & --- & 1 & 9 & 31 & --- \\
\hline aabbDDee--. & -.- & 0 & 0 & 0 & ---- & 0 & 4 & 13 & ---- & 1 & 9 & 31 & $\cdots$ \\
\hline aabbddEE--- & --- & 0 & 0 & 0 & $-\cdots$ & 0 & 4 & 13 & 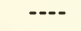 & 1 & 9 & 31 & $-\cdots$ \\
\hline Aa BbDdee--- & $\cdots$ & 63 & 16 & 3 & --- & 249 & 122 & 60 & $\ldots$ & 252 & 157 & 116 & --- \\
\hline AaBbddEe--- & $\cdots$ & 63 & 16 & 3 & $\cdots$ & 249 & 122 & 60 & --- & 252 & 157 & 116 & ---- \\
\hline AabbDdEe--- & $-\cdots$ & 63 & 16 & 3 & --- & 249 & 122 & 60 & ---- & 252 & 157 & 116 & --.- \\
\hline aABbDdEe--- & --- & 63 & 16 & 3 & --- & 249 & 122 & 60 & --- & 252 & 157 & 116 & --- \\
\hline ABBbddee--- & --. & 62 & 39 & 15 & $\cdots$ & 248 & 308 & 280 & --- & 249 & 347 & 418 & --- \\
\hline AabbDdee--- & -..- & 62 & 39 & 15 & $\cdots$ & 248 & 308 & 280 & --- & 249 & 347 & 418 & --- \\
\hline AabbddEe--- & $\cdots$ & 62 & 39 & 15 & $\ldots$ & 248 & 308 & 280 & --- & 249 & 347 & 418 & --- \\
\hline a ABbDdee--- & ---- & 62 & 39 & 15 & --- & 248 & 308 & 280 & --- & 249 & 347 & 418 & $\cdots$ \\
\hline QABbddEe-- &.-- & 62 & 39 & 15 & -.-- & 248 & 308 & 280 & -..- & 249 & 347 & 418 & $\cdots$ \\
\hline aabbDdEe--- & ---- & 62 & 39 & 15 & --- & 248 & 308 & 280 & $-\cdots$ & 249 & 347 & 418 & --- \\
\hline Aabbddee--- & $\cdots$ & 62 & 109 & 93 & -... & 247 & 859 & 1,555 & -.-- & 248 & 904 & 193 &.-- \\
\hline aa Bbddee --- &.-- & 62 & 109 & 93 & $\cdots$ & 247 & 859 & 1,555 & --- & 248 & 904 & 193 & --- \\
\hline aabbDdee--- & $\cdots$ & 62 & 109 & 93 & -... & 247 & 859 & 1,555 & --- & 248 & 904 & 193 & $\cdots$ \\
\hline aabbddEe--- & -... & 62 & 109 & 93 & $-\ldots$ & 247 & 859 & 1,555 & ---- & 248 & 904 & 193 & $--\cdot$ \\
\hline aabbddee-..- & -w-- & 62 & 348 & 812 & $\ldots$ & 247 & 2,742 & 12,781 & ---- & 247 & 2,798 & 13,927 & --- \\
\hline Total-. 1, & .02 & 041 & 1,094 & 1,291 & 2.015 & 4.091 & 8,672 & 21,062 & 2,040 & 4,247 & 9,449 & 25,011 & 4,030 \\
\hline
\end{tabular}




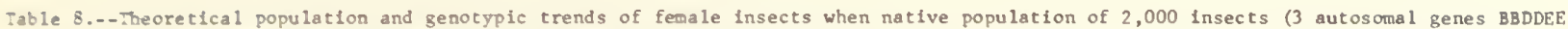
plus 1 sex-linked gene Ad; fem le is heterogametic) is overflooded by 198,000 or 396,000 partially sterlle insects (aabbddee and a-bbddee) during parental, $F_{1}, F_{2}$, and $F_{3}$ generations. Matings between partially sterlle insects are assumed to yleld no offspring

198,000 released with indicated sterility, increase, and generation

Cenotype

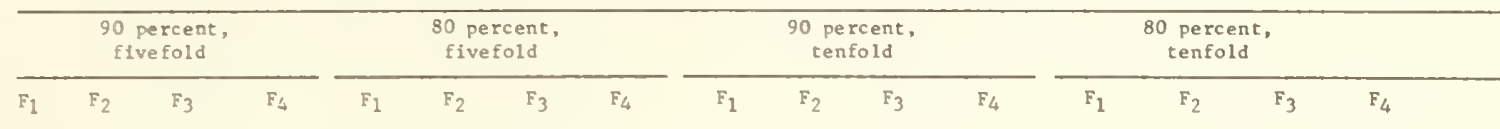

A B3DDES_.- $50 \quad 1 \quad 000$

A BBDDEe--- $-\cdots-1$

A BBDCEE-.. -..

(1)

A B3DDeE-.- -.--

A BBdCEE-.-

A BBDdEE-.. -..-

A BbLDEN-.- …

A BBDCEE-.. -..-

A BBDdee--- --.-

A BzddDe-.- -... 1

A BbDDee--- --.-

A BoddEZ-..

A bBDDZe--- -.--

A bbJez-..-

$\begin{array}{ccc}50 & 3 & 0 \\ \ldots \ldots & 4 & 1 \\ \ldots- & 4 & 1 \\ \ldots- & 4 & 1 \\ \ldots- & 2 & 1 \\ \ldots- & 2 & 1 \\ \ldots & 2 & 1\end{array}$

$\begin{array}{lcc}0 & 100 & 8 \\ 0 & \cdots- & 10 \\ 0 & \cdots- & 10 \\ 0 & \cdots- & 10\end{array}$

$\begin{array}{ll}0 & 0 \\ 0 & 0\end{array}$

0

a BBDDEE -.. -.. 1

$\triangle$ BbDdEe-- $495 \quad 59$

a BBDCE -. -.. 1

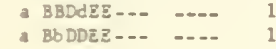

A Bbddee-.. -..- 0

$\begin{array}{lll}\text { A bbDdee -. } & \ldots & \\ \text { A bbddee -.. } & -. .- & 0\end{array}$

A BbDdee -.- -.. 32

A Bbddže -.. -... 32

A bbDdRe -.. -... 32

a BBDDee -. -... 0

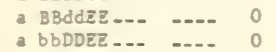

a BBDCZE-.. -... 2

a BbDDEe-.-

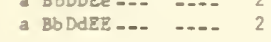

A Boddee... -... 31

A bbDdee... -... 31

A bbddEe... .... 31

a BBDdee ... .... 1

a BBddze ... ....

a BbDDee... ....

a BoddEZ... ....

a bbDDEe ... -..

a BbDCje - $495 \quad 120$

a BBddee ... .... 0

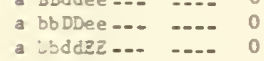

A bbddee-.. -... 31

a BbDcee -.. -... 93

a BbddZe-.. -... 93

a bbDdRe ... .... 93

a Bbddee... -... 93

a bbDdee ... $\ldots . . .993$

a bbddze -.. 2... 93

0

$\begin{array}{lll}\cdots- & 7 & 3 \\ \cdots & 7 & 3 \\ \cdots & 7 & 3\end{array}$

$\begin{array}{lll}\ldots- & 3 & 3 \\ \ldots- & 3 & 3 \\ \ldots- & 3 & 3 \\ \cdots- & 3 & 3 \\ \cdots & 3 & 3\end{array}$

+.-.

$990 \quad 183 \quad 51$

$\begin{array}{lll}\ldots \ldots & 3 & 1 \\ \ldots \ldots & 3 & 1 \\ \ldots \ldots & 3 & 1\end{array}$

$\begin{array}{ll}\ldots- & 2 \\ \cdots & 2 \\ \cdots & 2\end{array}$

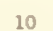

10
10
10

0
0
0

0
1
1

$\begin{array}{ll} & 127 \\ \cdots-- & 127 \\ \cdots-- & 127\end{array}$

$\begin{array}{ll}\ldots & 2 \\ \cdots & 2 \\ \cdots & 2\end{array}$

$\begin{array}{ll}\ldots & 6 \\ \ldots & 6 \\ \ldots- & 6\end{array}$

$\begin{array}{llll}--- & 124 & 173 & 201 \\ -\ldots- & 124 & 173 & 201\end{array}$

$\begin{array}{llll}\ldots+- & 124 & 173 & 201 \\ \ldots-- & 124 & 173 & 201\end{array}$

$\begin{array}{llll}-\ldots & 3 & 9 & 12\end{array}$

$+\infty$

- -

-

$--$

$\begin{array}{llll}990 & 425 & 198 & 124\end{array}$

-.-

57

53

53

53

$13 \quad 113$

$\begin{array}{ll}13 & 113 \\ 13 & 113\end{array}$

$404 \quad 884$

$\begin{array}{rr}404 & 884 \\ 1,161 \quad 1,386\end{array}$ 


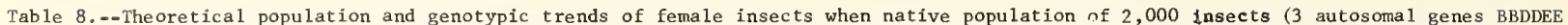
plus 1 sex-linked gene AA; female is heterogametic) is overflooded by 198,000 or 396,000 partially sterile insects (aabbddee and a-bbddee) during parental, $F_{1}, F_{2}$, and $F_{3}$ generations. Matings between partially sterile insects are assumed to yield no offspring-Continued

\begin{tabular}{|c|c|c|c|c|c|c|c|c|c|c|c|c|c|c|c|c|}
\hline \multirow{3}{*}{ Genotype } & \multicolumn{16}{|c|}{396,000 released with indicated sterility, increase, and generation } \\
\hline & \multirow[b]{2}{*}{$\mathrm{F}_{1}$} & \multicolumn{3}{|c|}{$\begin{array}{l}90 \text { percent, } \\
\text { fivefold }\end{array}$} & \multicolumn{4}{|c|}{$\begin{array}{l}80 \text { percent, } \\
\text { fivefold }\end{array}$} & \multicolumn{4}{|c|}{$\begin{array}{l}90 \text { percent, } \\
\text { tenfold }\end{array}$} & \multicolumn{4}{|c|}{$\begin{array}{l}80 \text { percent, } \\
\text { tenfold }\end{array}$} \\
\hline & & $\mathrm{F}_{2}$ & $\mathrm{~F}_{3}$ & $\mathrm{~F}_{4}$ & $\mathrm{~F}_{1}$ & $F_{2}$ & $\mathrm{~F}_{3}$ & $\mathrm{~F}_{4}$ & $\mathrm{~F}_{1}$ & $\mathrm{~F}_{2}$ & $\mathrm{~F}_{3}$ & $\mathrm{~F}_{4}$ & $\mathrm{~F}_{1}$ & $\mathrm{~F}_{2}$ & $F_{3}$ & $\mathrm{~F}_{4}$ \\
\hline A BBDDEE--- & 25 & 0 & 0 & 0 & 25 & 1 & 0 & 0 & 50 & 3 & 0 & 0 & 50 & 8 & 2 & 2 \\
\hline A BBDDEe--- & --- & 1 & 0 & 0 & $-\ldots$ & 2 & 0 & 0 & --- & 4 & 1 & 0 &.-- & 14 & 9 & 12 \\
\hline A BBDdEE $=--$ & --- & 1 & 0 & 0 & $-\infty$ & 2 & 0 & 0 & $\ldots$ & 4 & 1 & 0 & --- & 14 & 9 & 12 \\
\hline A BbDDEE--- & $-\cdots$ & 1 & 0 & 0 & --- & 2 & 0 & 0 & --- & 4 & 1 & 0 & $-\infty$ & 14 & 9 & 12 \\
\hline A BBDDee--- & --- & 0 & 0 & 0 & $=--$ & 1 & 0 & 0 & --- & 2 & 1 & 0 & --- & 6 & 10 & 20 \\
\hline A BBddEE--- & $-\infty$ & 0 & 0 & 0 & --- & 1 & 0 & 0 & $-\infty$ & 2 & 1 & 0 &.-- & 6 & 10 & 20 \\
\hline A bbDDEE--- & $\cdots$ & 0 & 0 & 0 & $-\infty-$ & 1 & 0 & 0 & --- & 2 & 1 & 0 & --- & 6 & 10 & 20 \\
\hline A BBDdEe--- & --- & 1 & 0 & 0 & $\ldots$ & 3 & 1 & 0 & --- & 7 & 3 & 1 & --- & 26 & 44 & 90 \\
\hline A BbDDEe--- & --- & 1 & 0 & 0 & -..- & 3 & 1 & 0 & --- & 7 & 3 & 1 & $\ldots$ & 26 & 44 & 90 \\
\hline A BbDdEE--- & --- & 1 & 0 & 0 & --- & 3 & 1 & 0 & --- & 7 & 3 & 1 & --- & 26 & 44 & 90 \\
\hline A BBDdEE--- & $-\infty-$ & 0 & 0 & 0 & $\ldots$ & 2 & 2 & 1 & --- & 3 & 4 & 3 & --- & 12 & 51 & 171 \\
\hline A BBddEe-.- & -- & 0 & 0 & 0 & $\ldots$ & 2 & 2 & 1 & $\ldots$ & 3 & 4 & 3 & $-\ldots$ & 12 & 51 & 171 \\
\hline A BbDDee--- & $\ldots$ & 0 & 0 & 0 & $\ldots$ & 2 & 2 & 1 & $-\ldots$ & 3 & 4 & 3 &.-- & 12 & 51 & 171 \\
\hline A BbddEE .- & --- & 0 & 0 & 0 & $-\infty$ & 2 & 2 & 1 & --- & 3 & 4 & 3 &.-- & 12 & 51 & 171 \\
\hline A bbDDEe-- & $-\ldots$ & 0 & 0 & 0 & --- & 2 & 2 & 1 & $\ldots$ & 3 & 4 & 3 & -- & 12 & 51 & 171 \\
\hline A bbDdEE-.- & --- & 0 & 0 & 0 & $\cdots$ & 2 & 2 & 1 & --- & 3 & 4 & 3 & --- & 12 & 51 & 171 \\
\hline a BBDDEE_-- & $-\cdots$ & 0 & 0 & 0 & --- & 1 & 0 & 0 & $\cdots$ & 2 & 0 & 0 & --- & 7 & 4 & 5 \\
\hline A BbDdEe--- & 497 & 45 & 4 & 0 & 995 & 154 & 31 & 9 & 995 & 186 & 53 & 23 & 1,990 & 637 & 460 & 957 \\
\hline a BBDDEa.-- & $\ldots$ & 0 & 0 & 0 & --- & 2 & 1 & 0 & $\ldots$ & 3 & 2 & 1 & $-\ldots$ & 13 & 20 & 40 \\
\hline a BBDdEE- - & --- & 0 & 0 & 0 & --- & 2 & 1 & 0 & $-\ldots$ & 3 & 2 & 1 & --- & 13 & 20 & 40 \\
\hline a BbDDEE $=-$ & --- & 0 & 0 & 0 & --- & 2 & 1 & 0 & --- & 3 & 2 & 1 & --- & 13 & 20 & 40 \\
\hline A BBddee-- & --- & 0 & 0 & 0 & --- & 1 & 2 & 2 & $\ldots$ & 2 & 5 & 7 & --- & 6 & 66 & 373 \\
\hline A bbDDee -.- & --- & 0 & 0 & 0 & $-\cdots$ & 1 & 2 & 2 & --- & 2 & 5 & 7 & $\ldots$ & 6 & 66 & 373 \\
\hline A bbddEE --- & --- & 0 & 0 & 0 & --- & 1 & 2 & 2 & --- & 2 & 5 & 7 & --- & 6 & 66 & 373 \\
\hline A BbDdee --- & 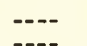 & & $\begin{array}{l}8 \\
8\end{array}$ & & $-\cdots$ & $\begin{array}{l}126 \\
126\end{array}$ & 63 & 32 & --- & 129 & 84 & 63 & --- & 512 & 731 & 2,173 \\
\hline A BbddEe -.- & -..- & $\begin{array}{l}32 \\
32\end{array}$ & $\begin{array}{l}8 \\
8\end{array}$ & $\begin{array}{l}2 \\
2\end{array}$ & $-\cdots$ & $\begin{array}{l}126 \\
126\end{array}$ & 63 & 32 &.-- & 129 & 84 & 63 & $--\infty$ & 512 & 731 & 2,173 \\
\hline A bbDdEe --- & -+- & & 8 & 2 & --- & 126 & 63 & 32 & --- & 129 & 84 & 63 & --- & 512 & 731 & 2,173 \\
\hline a BBDDee --- & -- & 0 & 0 & 0 & $\ldots$ & 1 & 1 & 0 & ---- & 2 & 2 & 1 & $\ldots$ & 6 & 2 & 77 \\
\hline a BBddEE -.- & --- & 0 & 0 & 0 & --- & 1 & 1 & 0 & $\ldots$ & 2 & 2 & 1 & -- & 6 & 2 & 77 \\
\hline a bbDDEE --- & --- & 0 & 0 & 0 & --- & 1 & 1 & 0 & --- & 2 & 2 & 1 & --- & 6 & 2 & 77 \\
\hline a BBDdEe --- & --- & 1 & 0 & 0 & $\ldots$ & 3 & 3 & 2 & $-\cdots$ & 6 & 8 & 6 & $-\infty$ & 25 & 105 & 353 \\
\hline a BbDDEe --- & $\ldots$ & 1 & 0 & 0 & --- & 3 & 3 & 2 & --- & 6 & 8 & 6 & --- & 25 & 105 & 353 \\
\hline a BbDdEE_-- & --- & 1 & 0 & 0 & --- & 3 & 3 & 2 & --- & 6 & 8 & 6 & --- & 25 & 105 & 353 \\
\hline A Bbddee.-- & --- & 31 & 20 & 8 & -... & 125 & 157 & 144 & -.- & 126 & 180 & 220 & $\ldots$ & 500 & 1,502 & 5,992 \\
\hline A bbDdee & $-\cdots$ & 31 & 20 & 8 & --- & 125 & 157 & 144 & $=\ldots$ & 126 & 180 & 220 & - & 500 & 1,502 & 5,992 \\
\hline A bbddEe...- & $-\cdots$ & 31 & 20 & 8 & $\cdots$ & 125 & 157 & 144 & $m$ & 126 & 180 & 220 & --- & 500 & 1,502 & 5,992 \\
\hline a BBDdee...- & --- & 0 & 0 & 0 & $\ldots$ & 2 & 4 & 5 & ---- & 3 & 9 & 14 & -... & 12 & 134 & 762 \\
\hline a BBddEe & $-\ldots$ & 0 & 0 & 0 & --- & 2 & 4 & 5 & $\ldots$ & 3 & 9 & 14 & $\ldots$ & 12 & 134 & 762 \\
\hline a BbDDee - -- & --- & 0 & 0 & 0 & $\ldots$ & 2 & 4 & 5 & $\cdots$ & 3 & 9 & 14 &.--- & 12 & 134 & 762 \\
\hline a BbddEE &.-- & 0 & 0 & 0 & $\ldots$ & 2 & 4 & 5 & $\ldots$ & 3 & 9 & 14 & $\ldots$ & 12 & 134 & 762 \\
\hline a bbDDEe_..- &.-- & 0 & 0 & 0 & $-\ldots$ & 2 & 4 & 5 &.-- & 3 & 9 & 14 & $-\ldots$ & 12 & 134 & 762 \\
\hline a bbDdEE_... & --- & 0 & 0 & 0 & -- & 2 & 4 & 5 & --- & 3 & 9 & 14 & --- & 12 & 134 & 762 \\
\hline a BbDdEe_.. 4 & 497 & 107 & 20 & 3 & 995 & 400 & 152 & 69 & 995 & 432 & 205 & 137 & 1,990 & 1,611 & 1,713 & 4,596 \\
\hline a BBddee --- & $\ldots$ & 0 & 0 & 0 & $\cdots$ & 1 & 1 & 15 & $-\ldots$ & 2 & 13 & 37 & --- & 6 & 184 & 187 \\
\hline a bbDDee --- & --- & 0 & 0 & 0 & -... & 1 & 1 & 16 & --- & 2 & 13 & 37 & -- & 6 & 184 & 187 \\
\hline a bbddEE -.- & $--\infty$ & 0 & 0 & 0 & $\cdots$ & 1 & 1 & 15 & $\cdots$ & 2 & 13 & 37 & $m$ & 6 & 184 & 187 \\
\hline A bbddee --- & --- & 31 & 55 & 47 & --- & 124 & 434 & 790 & --- & 125 & 462 & 997 & --- & 494 & 3,683 & 20,697 \\
\hline a BbDdee --- & $-\infty$ & 94 & 47 & 17 & $\cdots$ & 372 & 369 & 310 & --- & 375 & 425 & 476 & $-\infty$ & 1,487 & 3,466 & 12,633 \\
\hline a BbddEe-_-- & $-\infty$ & 94 & 47 & 17 &.-- & 372 & 369 & 310 & -- & 375 & 425 & 476 & --- & 1,487 & 3,466 & 12,633 \\
\hline a bbDdEe--- & --- & 94 & 47 & 17 & --- & 372 & 369 & 310 & ---- & 375 & 425 & 476 & --- & 1,487 & 3,466 & 12,633 \\
\hline a Bbddee--- & -.- & 93 & 128 & 101 & $\ldots$ & 371 & 1,013 & 1,695 & -..- & 372 & 1,078 & 2,141 & -- & 1,475 & 8,499 & 43,640 \\
\hline a bbDdee--- & $-\ldots$ & 93 & 128 & 101 & --- & 371 & 1,013 & 1,695 & --- & 372 & 1,078 & 2,141 & $-\infty$ & 1,475 & 8,499 & 43,640 \\
\hline a bbddEe--- & --- & 93 & 128 & 101 & --- & 371 & 1,013 & 1,695 & & 372 & 1,078 & 2,141 & --- & 1,475 & 8,499 & 43,640 \\
\hline a bbddee--- & $-\infty$ & 93 & 402 & 858 & $\ldots-$ & 370 & 3,171 & 13,559 & $\ldots$ & 371 & 3,250 & 14,893 & ---- & 1,469 & 24,962 & 227,215 \\
\hline
\end{tabular}


Table 9.--Theoretical population and genotypic trends when native population of 2,000 insects (aa) is overflooded by 198,000 or 396,000 partially sterile insects (AA) during parental, $F_{1}, F_{2}$, and $F_{3}$ generations. Matings between partially sterfle insects are assumed to yleld offspring proportionate to sterflity level

Genotvpe

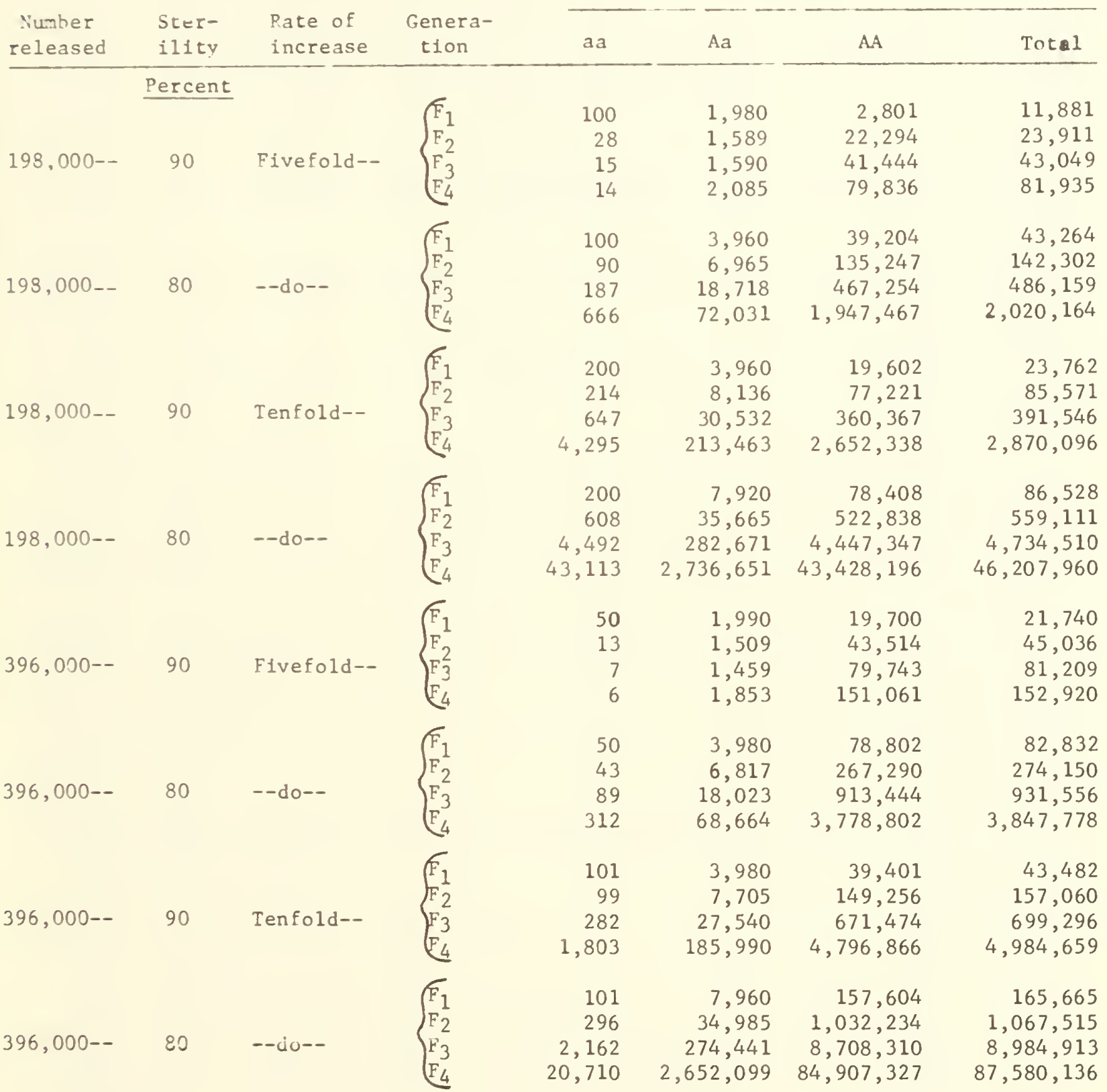


MISC. PUB. 1178, U.S. DEPT. OF AGRICULTURE

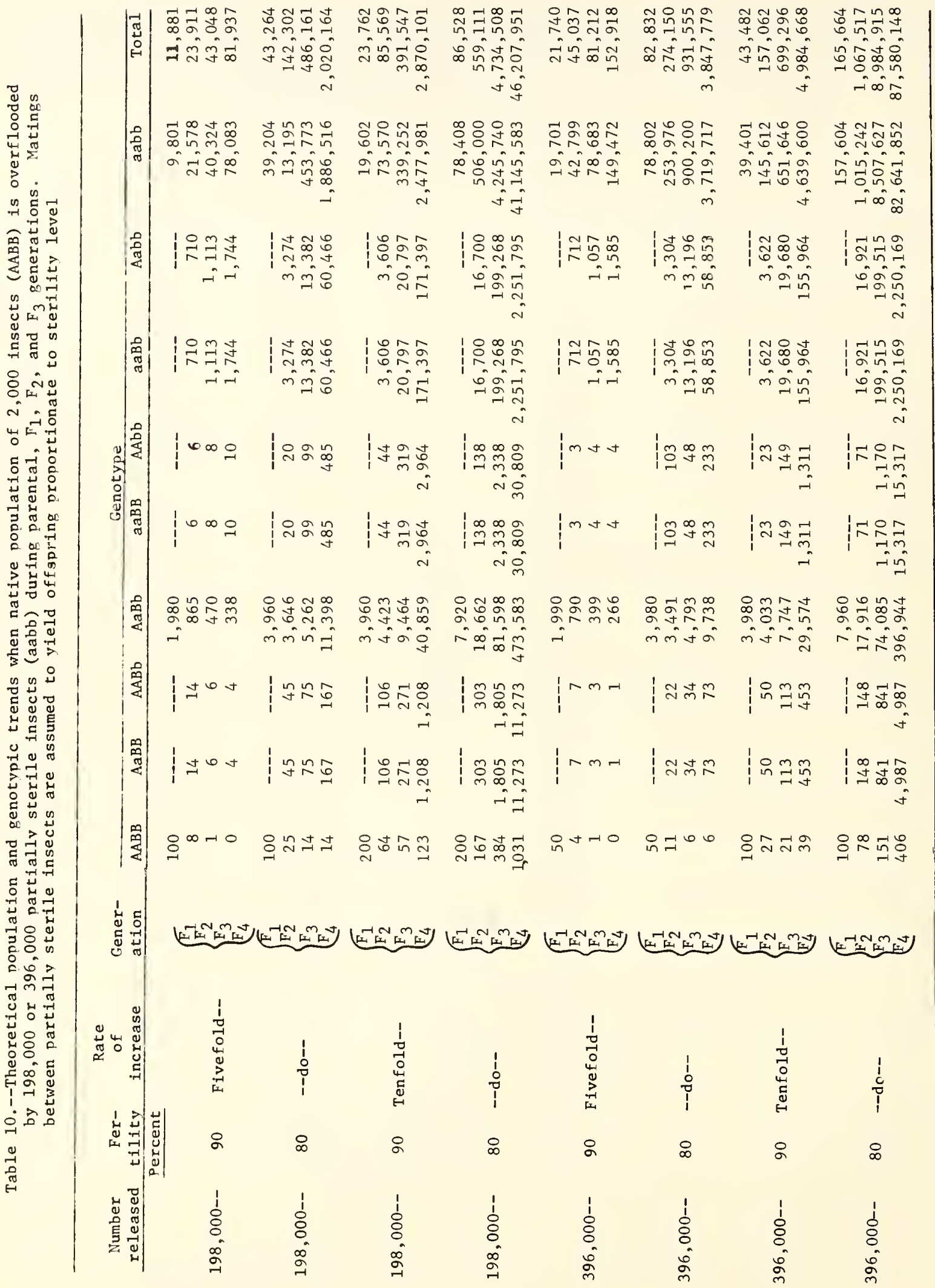




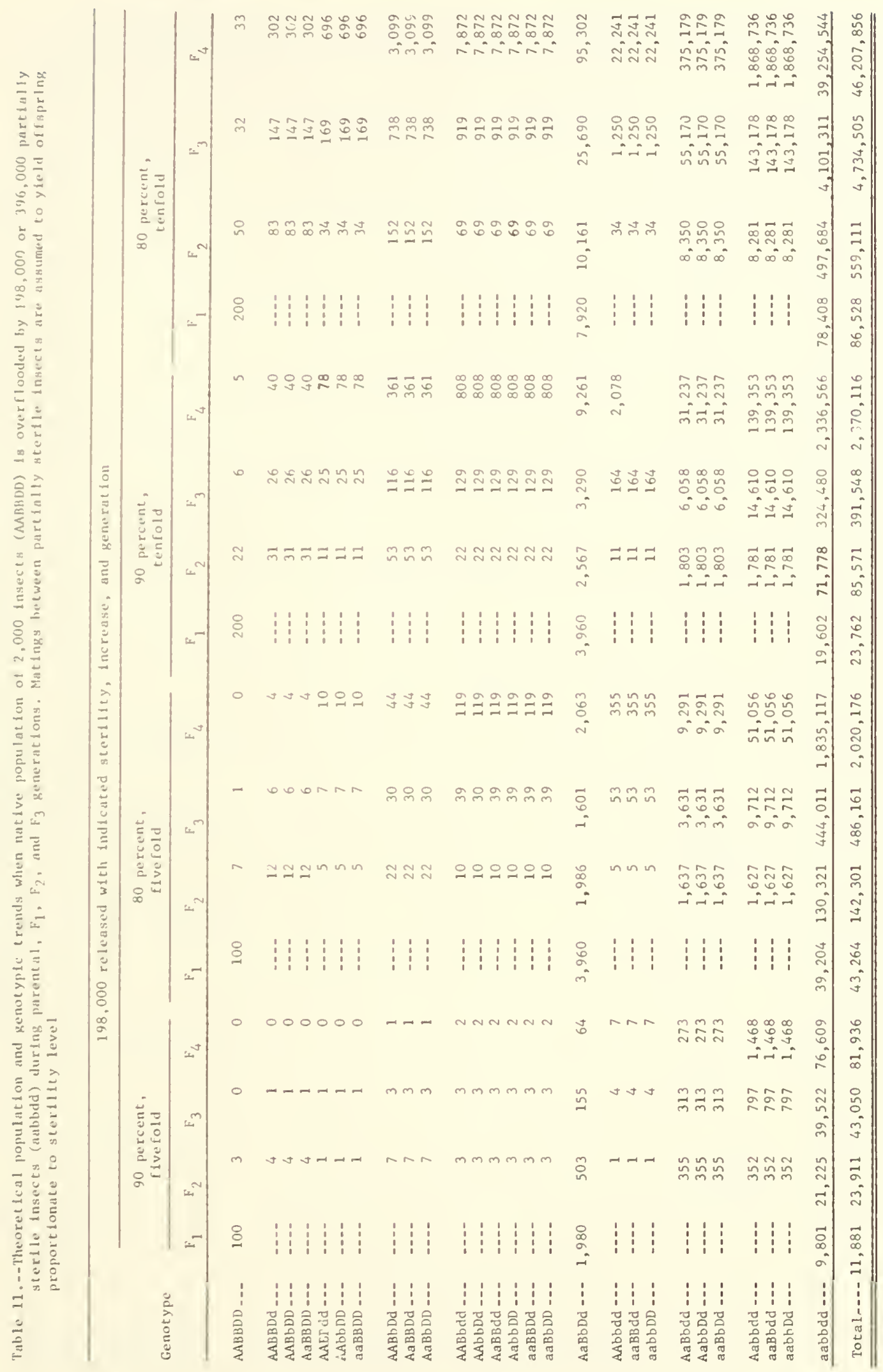


MISC. PUB. 1178, U.S. DEPT. OF AGRICULTURE

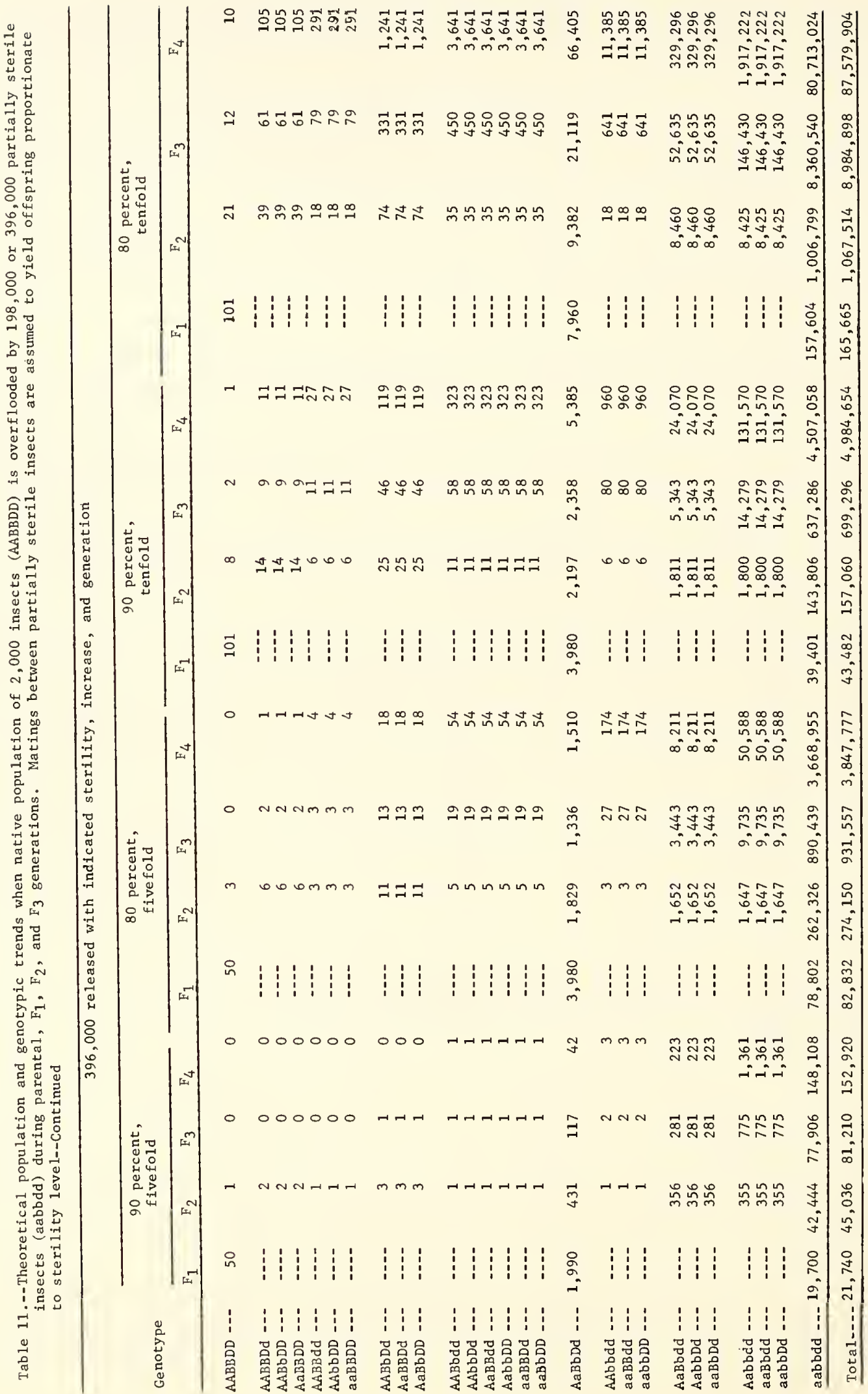


-able 12...-Teoretsial population and genotypic trends when native populstion of 2,000 1nsects (AABBDDEE) 1s overflooded by 198,000 or 196,000 partis1ly sterile insects abbddee) during parental, $F_{1}, F_{2}$, and $F_{3}$ generations. Matings between partially sterile insects are assumed to yield of fspring proportionate to sterility level

\begin{tabular}{|c|c|c|c|c|c|c|c|c|c|c|c|c|c|c|}
\hline Genotupe & & $\begin{array}{l}90 \\
\mathrm{fx}\end{array}$ & $\begin{array}{l}\text { rceet, } \\
\text { sold }\end{array}$ & & & $\begin{array}{l}80 \mathrm{p} \\
\text { fiv }\end{array}$ & $\begin{array}{l}\text { rercent. } \\
\text { efold. }\end{array}$ & & & $\begin{array}{l}90 \text { per } \\
\text { tenf }\end{array}$ & $\begin{array}{l}\text { cent, } \\
\text { fold }\end{array}$ & & & $\begin{array}{l}80 \text { per } \\
\text { tenf }\end{array}$ \\
\hline & $\Sigma_{1}$ & $E_{2}$ & $\mathrm{~F}_{3}$ & $\mathrm{~F}_{-}$ & $E_{1}$ & $\mathrm{~F}_{2}$ & $\mathrm{~F}_{3}$ & $F_{4}$ & $F_{1}$ & ${ }^{E_{2}}$ & $F_{3}$ & $F_{4}$ & $F_{1}$ & $\mathrm{E}_{2}$ \\
\hline M3золет ... & 100 & 1 & 0 & 0 & 100 & 3 & 0 & 0 & 200 & 9 & 1 & 0 & 200 & 17 \\
\hline Mu3vose ... & .... & 1 & 0 & c & --- & 4 & 1 & 0 & $-\ldots$ & 10 & 3 & 2 & $-\ldots$ & 24 \\
\hline M33pez3 -.- & -... & 1 & 0 & 0 & --- & 4 & 1 & 0 & $\cdots$ & 10 & 3 & 2 & $\cdots$ & 24 \\
\hline$\mu 13 b 0033 \ldots$ & $-\ldots$ & 1 & 0 & 0 & -... & 8 & 1 & 0 & --- & 10 & 3 & 2 & $-\ldots$ & 24 \\
\hline MBSDDE -.. & $\ldots$ & 1 & 0 & 0 & --- & 4 & 1 & 0 & --- & 10 & 3 & 2 & $\cdots$ & 24 \\
\hline Alasndee -.. & $-\infty$ & O & 0 & 0 & $\ldots$ & 1 & 1 & 0 & --- & 3 & 2 & 3 & $\ldots$ & 9 \\
\hline A3Bdd5 ... & $\ldots$ & o & 0 & 0 & $\ldots$ & 1 & 1 & 0 & -...- & 3 & 2 & 3 & --- & 9 \\
\hline MAbbDoes -.- & -..- & 0 & 0 & 0 & $\ldots$ & 1 & 1 & 0 & $-\cdots$ & 3 & 2 & 3 & - & 9 \\
\hline Aasadoc? -.- & $\ldots$ & 0 & 0 & c & $-\cdots$ & 1 & 1 & 0 & $-\cdots$ & 3 & 2 & 3 & $\cdots$ & 9 \\
\hline Mas Dd?e -.. & -... & 2 & 0 & 0 &..-- & 6 & 3 & 1 & $=-$. & 16 & 12 & 14 & $-\infty$ & 41 \\
\hline nesscze ... & --.- & 2 & 0 & 0 & --- & 6 & 3 & 1 & $\ldots$ & 16 & 12 & 14 & -...- & 41 \\
\hline MUBDDSE -.. & -...- & 2 & 0 & 0 & --- & 6 & 3 & 1 & $-\ldots$ & 16 & 12 & 14 & --- & 41 \\
\hline Lusooze -.- & $-\infty$ & 2 & 0 & 0 & $\ldots$ & 6 & 3 & 1 & --- & 16 & 12 & 14 & --- & 41 \\
\hline Mas3dez $=$. & -.-. & 2 & 0 & 0 & --- & 6 & 3 & 1 & $-\cdots$ & 16 & 12 & 14 & $-\cdots$ & 41 \\
\hline MabsDoge -.- & $\cdots$ & 2 & 0 & 2 & $\cdots$ & 6 & 3 & 1 & --- & 16 & 12 & 14 & $\cdots$ & 41 \\
\hline Masdece ... & -.-- & 1 & 3 & 2 & $-\infty$ & 3 & 3 & 3 & $\ldots$ & 6 & 11 & 24 &.-- & 17 \\
\hline M33dede ..- & $-\cdots$ & 1 & 0 & 0 & -..- & 3 & 3 & 3 & $\ldots$ & 6 & 11 & 24 & $-\cdots$ & 17 \\
\hline w3bJdee ..- &..-- & 1 & 0 & 0 & -a.- & 3 & 3 & 3 & $\cdots$ & 6 & 11 & 24 & $\cdots$ & 17 \\
\hline Mobdez? -.. &..- & 1 & 0 & 2 & --- & 3 & 3 & 3 & ---- & 6 & 11 & 24 & --- & 17 \\
\hline MacbDose $=$. & $\ldots$ & 1 & 2 & 0 & --- & 3 & 3 & 3 & --- & 6 & 11 & 24 & -..- & 17 \\
\hline ALSBDCER =- & $--\infty$ & 1 & 0 & 0 & --- & 3 & 3 & 3 & $\cdots$ & 6 & 11 & 24 & $\ldots$ & 17 \\
\hline Aus abee -.- & $-\cdots$ & 1 & 0 & 0 & -- & 3 & 3 & 3 & $-\ldots$ & 6 & 11 & 24 & $\cdots$ & 17 \\
\hline MaBdd32 ... & $--\cdot$ & 1 & 0 & 0 & --- & 3 & 3 & 3 & $-\cdots$ & 6 & 11 & 24 & --- & 17 \\
\hline MbbDo:3 -.- & ---- & 1 & 0 & 0 & -... & 3 & 3 & 3 & -... & 6 & 11 & 24 & --- & 17 \\
\hline -433DDRe $=-$ & $-\ldots$ & 1 & 0 & 0 & $\ldots$ & 3 & 3 & 3 & --- & 6 & 11 & 24 & $\cdots$ & 17 \\
\hline - a $3300 \mathrm{CZZ}$... &..-- & 1 & D & 0 & $-\infty$ & 3 & 3 & 3 & $\ldots$ & 6 & 11 & 24 & $--\cdot$ & 17 \\
\hline a a BbDDe? =-- & -..- & 1 & 0 & 0 & -..- & 3 & 3 & 3 & $=-$ & 6 & 11 & 24 & $\cdots$ & 17 \\
\hline MBbDeZe & -... & 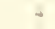 & 1 & 0 & $-\ldots$ & 11 & 12 & 12 & --- & 27 & 51 & & $\cdots$ & 76 \\
\hline $\operatorname{As} 3 \mathrm{C}^{3} \mathrm{e}$ &..- & $\rightarrow$ & 1 & 0 & -... & 11 & 12 & 12 & -... & 27 & 51 & 115 &..- & 76 \\
\hline A $3 b D 0 E$... & -... & - & 1 & 0 & $\ldots$ & 11 & 12 & 12 & --- & 27 & 51 & 115 &.--- & 76 \\
\hline$A 3 b D C Z 3 \ldots$ & $\ldots$ & + & 1 & 0 & --- & 11 & 12 & 12 & --- & 27 & 51 & 115 & $\cdots$ & 76 \\
\hline Musclee ... & $\ldots$ & 2 & 0 & 0 & $\ldots$ & 1 & 4 & 7 & --- & 3 & 12 & 51 & ---- & 9 \\
\hline AubbDsee ... & $\ldots$ & 0 & 0 & 0 & $\ldots$ & 1 & 4 & 7 & -- & 3 & 12 & 51 & $\cdots$ & 9 \\
\hline Aubdez? =.- & ...- & 0 & 0 & 0 & $\ldots$ & 1 & 4 & 7 & $\ldots$ & 3 & 12 & 51 & --- & 9 \\
\hline absoode ..- & -..- & 0 & 0 & 0 & $-\ldots$ & 1 & 4 & 7 & -... & 3 & 12 & 51 & $\ldots$ & 9 \\
\hline a $38 d 0 d z$ & -..- & 0 & 0 & 0 & --- & 1 & 4 & 7 & -... & 3 & 12 & 51 &..-- & 9 \\
\hline abbb0E ..- & --- & 0 & 0 & 0 & --- & 1 & 4 & 7 & $\ldots$ & 3 & 12 & 51 & $\ldots$ & 9 \\
\hline MabDdet ... & -...- & 1 & 1 & 1 &.--- & 5 & 15 & 31 & ---- & 11 & 53 & 233 & $\ldots$ & 34 \\
\hline Mubdabe ... & -... & 1 & 1 & 1 &.-- & s & is & 31 & - & 11 & 53 & 233 & $\ldots$ & 34 \\
\hline AubbDete ... & $-\ldots$ & 1 & 1 & 1 & $-\infty$ & 5 & 15 & 31 & $\ldots$ & 11 & 53 & 233 &..- & $3 / 4$ \\
\hline Aasadee ...- & -... & 1 & 1 & 1 & $\ldots$ & 5 & 15 & 31 & $\ldots$ & 11 & 53 & 233 & --- & 34 \\
\hline AnB̈BdARe -.. & $\ldots$ & 1 & 1 & 1 & $-\infty$ & 5 & 15 & 31 & $=-$. & 11 & 53 & 233 &.- & 34 \\
\hline As 3bDDee ... & -..- & 1 & 1 & 1 & -...- & 5 & 15 & 31 & $\ldots$ & ii & 53 & 233 & -- & 34 \\
\hline Mabddzz --- & $-\ldots$ & 1 & 1 & 1 & $\cdots$ & 5 & 15 & 31 & -..- & 11 & 53 & 233 & $\ldots$ & 34 \\
\hline AubbDoee .... & $\ldots$ & 1 & 1 & 1 & --- & 5 & 15 & 31 & $\ldots$ & ii & 53 & 233 & 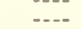 & 34 \\
\hline$\triangle a b b D C E Z$... & $\ldots$ & 1 & 1 & 1 & --- & $s$ & 15 & 31 &.-- & 11 & 53 & 233 & $-\ldots$ & 34 \\
\hline A.33Det2 ... & $\ldots$ & 1 & 1 & 1 & .... & 5 & 15 & 31 & -.-- & 11 & 53 & 233 & $-\ldots$ & 34 \\
\hline aA3bDoze ... & --.- & 1 & 1 & 1 & -..- & 5 & 15 & 31 & --- & 11 & 53 & 233 & $-\ldots$ & 34 \\
\hline aABSDCPB ... &.--- & 2 & 1 & 1 & $-\cdots$ & 5 & 15 & 31 & .... & 11 & 53 & 233 & $\ldots$ & 34 \\
\hline Ansbder... & $-\ldots$ & 1 & 2 & 2 & --- & 3 & 20 & 86 & $\ldots$ & 6 & 65 & 551 & -... & 17 \\
\hline MabbDee ... & $-\ldots$ & 2 & 2 & 2 & $\ldots$ & 3 & 20 & 86 &..- & 6 & 65 & 551 & 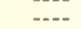 & 17 \\
\hline Mbbddze -.- & --- & 1 & 2 & 2 & .... & 3 & 20 & 86 &.-- & 6 & 65 & 551 &..- & 17 \\
\hline A33addee ...- &.-- & 1 & 2 & 2 &..-- & 3 & 20 & 86 & --.- & 6 & 65 & 551 & $\ldots$ & 17 \\
\hline AsbbDoce... & --- & 1 & 2 & 2 & -... & 3 & 20 & 86 & $\ldots$ & 6 & 65 & 551 & --- & 17 \\
\hline Aabbeczz -.- & $-\ldots$ & 1 & 2 & 2 & -... & 3 & 20 & 86 & $\ldots$ & 6 & 65 & 551 & --.- & 17 \\
\hline a a Bs Jee ... & --- & 1 & 2 & 2 & --- & 3 & 20 & 86 &..- & 6 & 65 & 551 & $--\cdot$ & 17 \\
\hline as 3 Bdd De .... & -...- & 1 & 2 & 2 & ---- & 3 & 20 & 86 &..- & 6 & 65 & 551 & $\ldots$ & 17 \\
\hline a a BoDDee .... &.-- & 1 & 2 & 2 &.- & 3 & 20 & 86 & $\ldots$ & 6 & 65 & 551 & $\ldots$ & 17 \\
\hline Aabbedzz ... & $\cdots$ & 1 & 2 & 2 & --- & 3 & 20 & 86 & $\ldots$ & 6 & 65 & 551 & $\ldots$ & 17 \\
\hline aAbbDDze -..- & $-\cdot-$ & 1 & 2 & 2 & .... & 3 & 20 & 86 & --- & 6 & 65 & 551 &..- & 17 \\
\hline AnSbDCZZ ... & $\ldots$ & 1 & 2 & 2 & -... & 3 & 20 & 86 & --- & 6 & 65 & 551 & $\cdots$ & 17 \\
\hline AasbDcze -. 1 & .980 & 322 & 59 & 14 & 3,960 & 1,156 & 543 & 435 & 3,960 & 1,639 & 1,306 & 2,448 & 7,920 & 5,910 \\
\hline Mubbdiee ... & $\ldots$ & 0 & 2 & 5 & .... & 1 & 29 & 262 & .... & 3 & 87 & 1,476 & $\ldots$ & 9 \\
\hline aajoddee ...- & $\ldots$ & 0 & 2 & 5 & --- & 1 & 29 & 262 &.-- & 3 & 87 & 1,476 &.-- & 9 \\
\hline aabbitee ... & --- & 0 & 2 & 5 &.--- & 1 & 29 & 262 & --- & 3 & 87 & 1,476 &.-- & 9 \\
\hline abbddze =.. & $\ldots$ & 0 & 2 & 5 &.-- & 1 & 29 & 262 & $\ldots$ & 3 & 87 & 1,476 & --- & 9 \\
\hline AaBbDiee -.. & $\ldots$ & 177 & 95 & & -..- & 819 & 1,045 & 1,616 & $\ldots$ & 902 & 1,932 & 6,698 & --.- & 4,175 \\
\hline Aa3bddRe ... &.- & 177 & 95 & 49 & $\ldots$ & 819 & 1,045 & 1,616 & $\ldots$ & 902 & 1,932 & 6,698 & ...- & 4.175 \\
\hline Aabb DCZe ... & $\ldots$ & 177 & 95 & 49 & -..- & 819 & 1,045 & 1,616 & $\ldots$ & 902 & 1,932 & 6,698 & $\ldots$ & $\begin{array}{l}4,175 \\
4,175\end{array}$ \\
\hline aaboscze -.. & -..- & 177 & 95 & 49 & 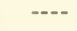 & 819 & 1,045 & 1,616 & $\cdots$ & 902 & 1,932 & 6,698 & $\cdots$ & 4,175 \\
\hline Aa3addee -.. & --- & 176 & 216 & 223 &..-- & 813 & 2,571 & 7,644 & --- & 891 & 4,073 & 24,306 & --- & \\
\hline AabbDdee ... &.-- & 176 & 216 & 223 & -... & 813 & 2,571 & 7,644 & $\ldots$ & 891 & 4,073 & 24,306 &.-- & $\begin{array}{l}4,140 \\
4,140\end{array}$ \\
\hline Aabbodze ... & $\ldots$ & 176 & 216 & 223 & $\cdots$ & 813 & 2,571 & 7,644 & $-\ldots$ & 891 & 4,073 & 24,306 & -..- & $\begin{array}{l}4,140 \\
4,140\end{array}$ \\
\hline a BbDiee ... & -... & 176 & 216 & 223 & $\cdots$ & 813 & 2,571 & 7,644 & $\cdots$ & 891 & 4,073 & 24,306 & $\ldots$ & 4,140 \\
\hline a $3 b d d 3 e$..- & --- & 176 & 216 & 223 & ---- & 813 & 2,571 & 7,644 & --- & 891 & 4,073 & 24,306 & $\cdots$ & 4,140 \\
\hline aabbDdZe $\ldots$ & --- & 176 & 216 & 223 & --- & 813 & 2,571 & 7,644 & $\cdots$ & 891 & 4,073 & 24,306 & $\cdots$ & 4,140 \\
\hline Aabbddee ... & ...- & 175 & 579 & 1,243 & -.. & 811 & 7,120 & 43,326 & $\cdots$ & 885 & 10,471 & 114,495 & $-\cdots$ & \\
\hline aaBbddee ... & -... & 175 & 579 & 1,243 & -... & 811 & 7,120 & 43,326 & $\ldots$ & 885 & 10,471 & 114,495 & --- & 4,123 \\
\hline aabbDce -.- & $\cdots$ & 175 & 579 & 1,243 & --- & 811 & 7,120 & 43,326 & --- & 885 & 10,471 & 114,495 & --- & 4,123 \\
\hline sabbddze -.- & -... & 175 & 579 & 1,243 & $\cdots$ & 811 & 7,120 & 43,326 & --- & 885 & 10,471 & 114,495 & --- & 4,123 \\
\hline aabucdee 9,8 & 801 & 1,049 & 38,941 & 75,360 & 39,204 & 129,508 & 436,859 & $, 791,515$ & 19,602 & 70.890 & 313.919 & 2.220 .572 & 78.408 & 493.552 \\
\hline tal-n-11, & 27 & 3,911 & 43,050 & 81,935 & 43,264 & 142,301 & 486,159 & $2,020,161$ & 23,762 & 85,571 & 391,545 & $2,870,090$ & 86.528 & $=559,110$ \\
\hline
\end{tabular}


Table 12.--Theoretical population and genotypic trends when native population of 2,000 insects (AAB8DDEE) 1s overflooded by 198,000 or 396,000 partially sterile insects (aabbdee) during parental, $F_{1}, F_{2}$ and $F_{3}$ generations. Matings between partially sterile insects are assumed to yleld of fspring proportionate to sterility level--Continued

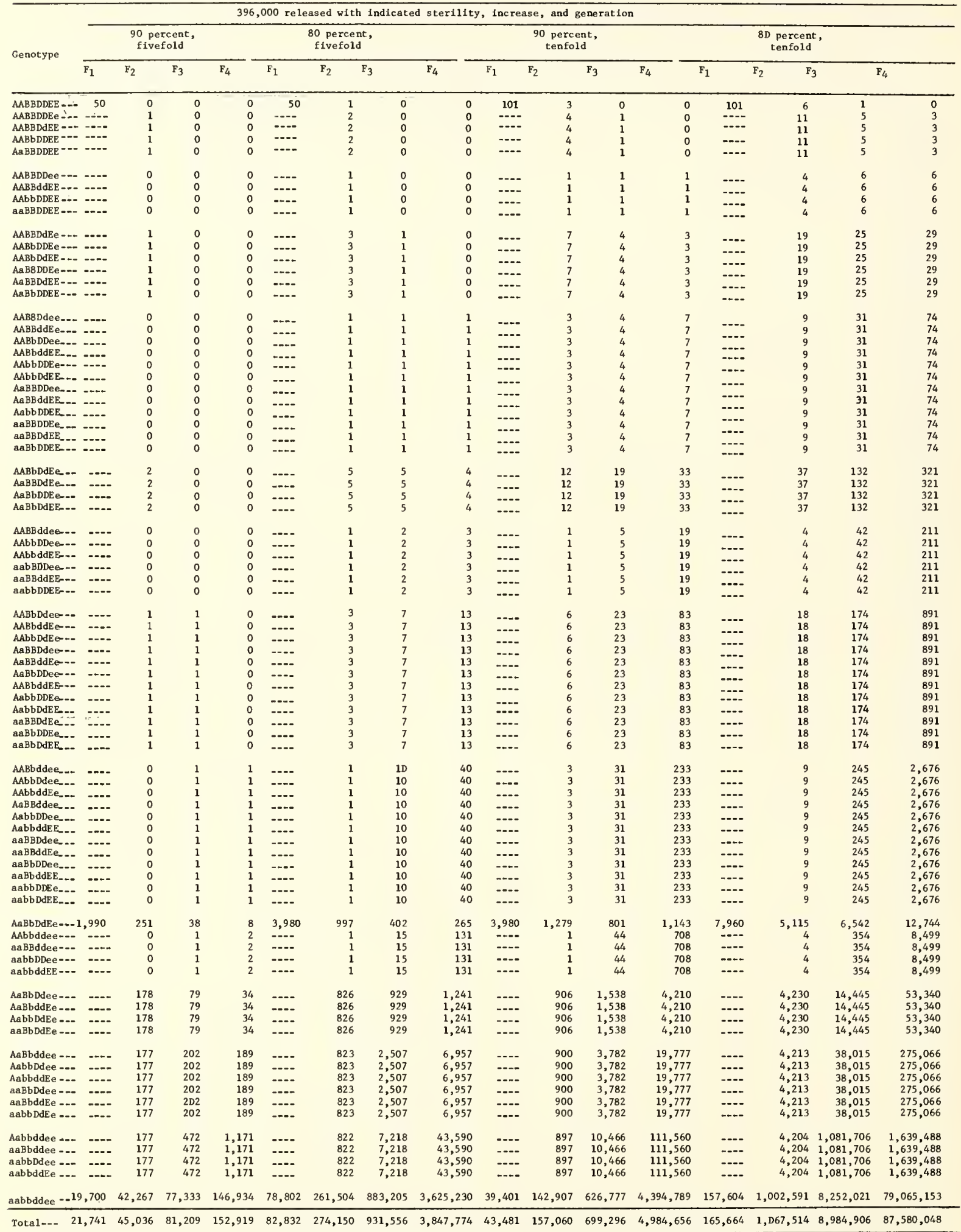




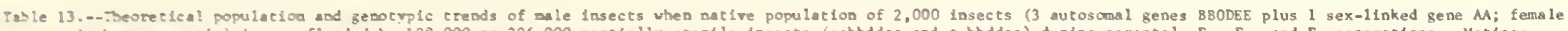

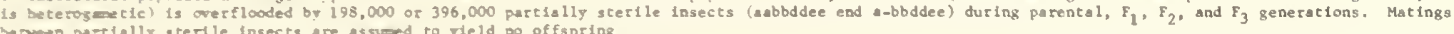

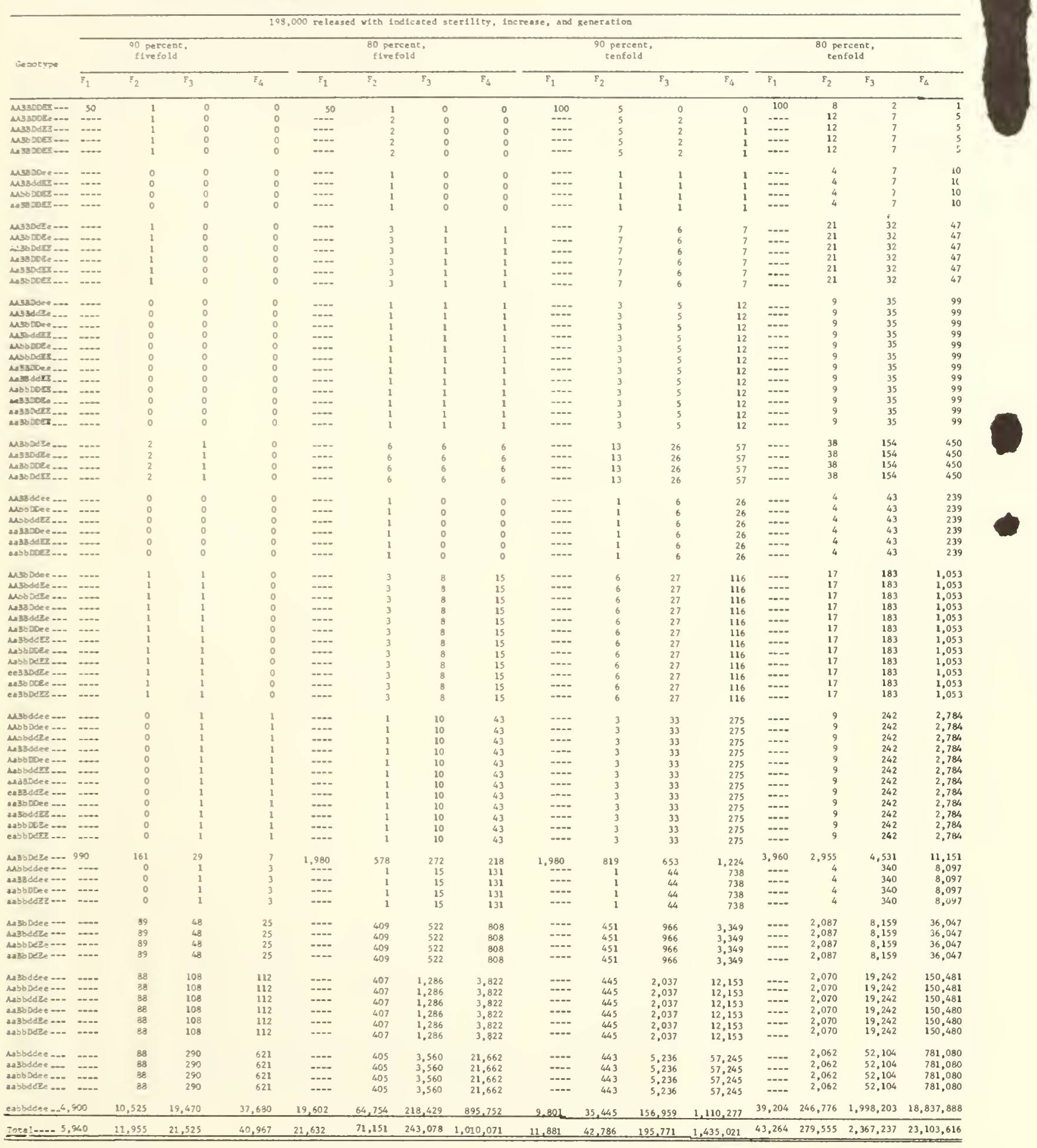


Table 13.--Theoretical population and genotypic trends of male insects when native population of 2,000 insects ( 3 autosomal genes BBDDEE plus 1 sex-1inked gene $\mathrm{AA}$; female is heterogametic) is overflooded by 198, D00 or 396,000 partially sterile insects (aabbddee and a-bbddee) during parental, $F_{1}$, $F_{2}$, and $F_{3}$ generations. Matings between partially sterile insects are assumed to yield no offspring--Continued

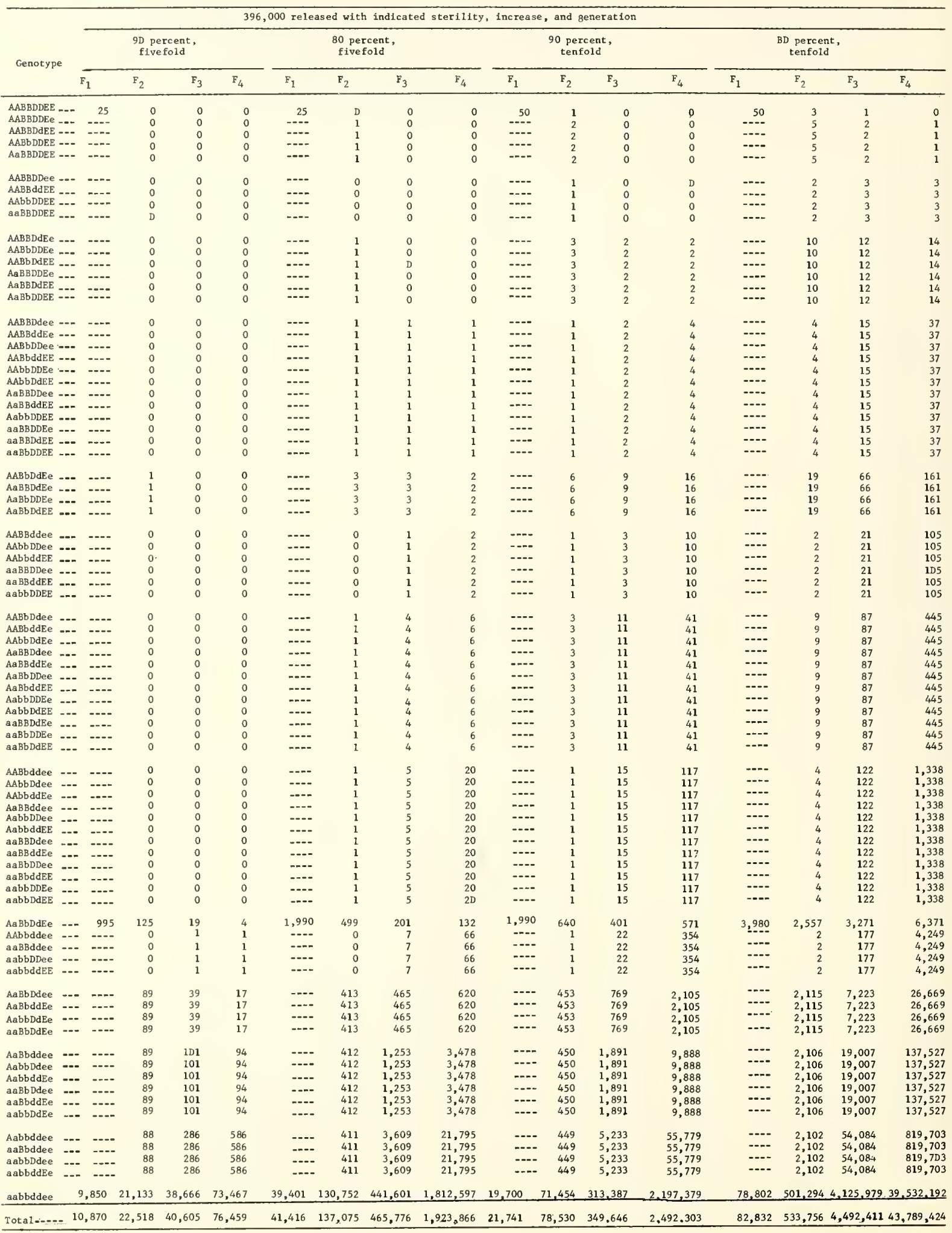




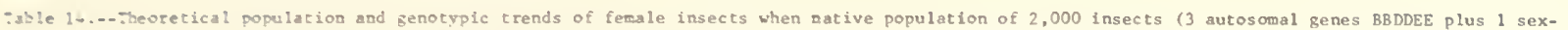
liaised gene At; fessle is heterogametic) is overflooded by 198,000 or 396,000 partially sterile insects (abbddee and a-bbddee) during parental,

$F_{1}$. F , and $F_{3}$ generations. Matings between partially sterlle insects are assumed to yield no offspring

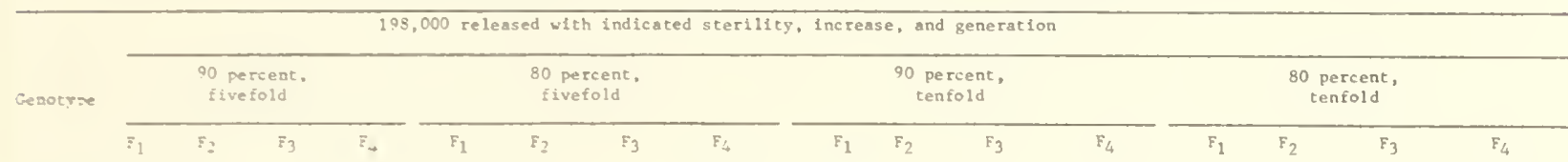

\begin{tabular}{|c|c|c|c|c|c|c|c|c|c|c|c|c|c|c|c|c|c|}
\hline A & 33205 & $\ldots 50$ & 60 & 0 & o & 50 & 2 & 0 & 0 & 100 & 7 & 1 & 1 & 100 & 15 & 6 & 3 \\
\hline A & SBDDEe &.- & $\ldots$ & 0 & 0 & - & 3 & 1 & 0 & $\ldots$ & 9 & 4 & 4 & --- & 22 & 23 & 29 \\
\hline A & ass Ddse & -- & -... & 0 & 0 & $\ldots-$ & 3 & 1 & 0 &.-- & 9 & 4 & 4 & .... & 22 & 23 & 29 \\
\hline A & 3SDJEE & -- & --- & 0 & 0 & $-\cdots$ & 3 & 1 & 0 & $\cdots$ & 9 & 4 & 4 & .... & 22 & 23 & 29 \\
\hline$A$ & gandee & --- & $\cdots$ & 0 & 0 & $\ldots$ & 1 & 1 & 1 & -... & 3 & 4 & 7 & .... & 9 & 2 & 60 \\
\hline A & 38 dCEE & -- & $\ldots$ & 0 & 0 & $\ldots$ & 1 & 1 & 1 & $\ldots$ & 3 & 4 & 7 & .... & 9 & 2 & 60 \\
\hline A & GDDDES & $\cdots$ & -... & 0 & 0 & -... & 1 & 1 & 1 & $\cdots$ & 3 & 4 & 7 & .... & 9 & 2 & 60 \\
\hline A & saDCEe & $\cdots$ & .... & 0 & 0 & $\ldots$ & 6 & 4 & 4 & $\ldots$ & 14 & 19 & 35 & -...- & 40 & 109 & 272 \\
\hline A & BbDOSe & --- & --- & 0 & 0 & $\ldots$ & 6 & 4 & 4 & $=-$. & 14 & 19 & 35 &.-- & 40 & 109 & 272 \\
\hline A & BbDCEE & 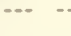 & $\cdots$ & 0 & 0 & --- & 6 & 4 & 4 & $\ldots$ & 14 & 19 & 35 & --- & 40 & 109 & 272 \\
\hline A & Badeee & $\cdots$ & $\cdots$ & 0 & 0 & $--\infty$ & 3 & 5 & 9 & $-\infty$ & 6 & 19 & 70 & --- & 17 & 126 & 625 \\
\hline A & $\begin{array}{l}\text { BBddice } \\
\text { Bb Doee }\end{array}$ & $\begin{array}{ll}--- & - \\
-- & -\end{array}$ & --- & 0 & 0 & 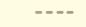 & 3 & 5 & 9 & $\ldots-$ & 6 & 19 & 70 & .... & 17 & 126 & 625 \\
\hline A & $\begin{array}{l}\text { 3b Doee } \\
\text { sbddEs }\end{array}$ & $\ldots-$ & 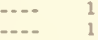 & 0 & 0 & ---- & 3 & 5 & 9 & $\ldots$ & 6 & 19 & 70 &.-- & 17 & 126 & 625 \\
\hline$A$ & $\begin{array}{l}\text { SbddEz } \\
\text { bDDJEe }\end{array}$ & $=-$ & -..- & 0 & 0 & $\ldots$ & 3 & 5 & 9 & --- & 6 & 19 & 70 & --- & 17 & 126 & 625 \\
\hline A & $\begin{array}{l}\text { DSDXEe } \\
\text { bbDCEz }\end{array}$ & $=-$ & 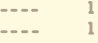 & 0 & 0 & $\ldots$ & 3 & 5 & 9 & $-\infty$ & 6 & 19 & 70 & --- & 17 & 126 & 625 \\
\hline A & & & -- & 0 & 0 & $\ldots$ & 3 & 5 & 9 & $\cdots$ & 6 & 19 & 70 & $\cdots$ & 17 & 126 & 625 \\
\hline a & 3BDDEE & --- & $\ldots$ & 0 & 0 & $-\ldots$ & 2 & 0 & 0 & $=--$ & 4 & 2 & 2 & --- & 10 & 11 & 13 \\
\hline A & BSDdEe & $--0-95$ & 82 & 15 & 4 & 990 & 295 & 142 & 115 & 990 & 423 & 352 & 669 & 1,980 & 1,515 & 2,420 & 6,026 \\
\hline a & BeDDE & $\cdots$ & $\cdots$ & a & 0 & $\cdots$ & 3 & 2 & 2 & -..- & 7 & 8 & 16 & --- & 19 & 50 & 122 \\
\hline a & BaDdEF & $\cdots$ & -... & 0 & 0 & -... & 3 & 2 & 2 & - - & 7 & 8 & 16 & $-\ldots$ & 19 & 50 & 122 \\
\hline a & BODDEE & --- & -- & 0 & 0 & $=--$ & 3 & 2 & 2 & .... & 7 & 8 & 16 & $\cdots$ & 19 & 50 & 122 \\
\hline A & Bsddee & --- & $\cdots$ & 1 & 1 & -..- & 1 & 7 & 25 &.-- & 3 & 22 & 163 & $\ldots$ & 9 & 164 & 1,631 \\
\hline $\begin{array}{l}\text { A } \\
\text { A }\end{array}$ & boblee & $\cdots$ & -. - & 1 & 1 & $\ldots$ & 1 & 7 & 25 & $\cdots$ & 3 & 22 & 163 & $-\infty$ & 9 & 164 & 1,631 \\
\hline A & & $\cdots--$ & --- & 1 & 1 & 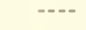 & 1 & 7 & 25 & $\cdots$ & 3 & 22 & 163 & --- & 9 & 164 & 1,631 \\
\hline $\begin{array}{l}A \\
A\end{array}$ & $\begin{array}{l}\text { Bb Deee } \\
\text { Bbddze }\end{array}$ & $\cdots$ & 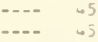 & 26 & 13 & -... & 207 & 269 & 419 & $\ldots$ & 231 & 510 & 1,791 & $\cdots$ & 1,061 & 4,263 & 19.077 \\
\hline A & bo dure. & --- & 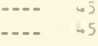 & 24 & 13 & --- & 207 & 269 & 419 & $\cdots$ & 231 & 510 & 1,791 & $\cdots$ & 1,061 & $\begin{array}{l}4,263 \\
4,263\end{array}$ & 19,077 \\
\hline a & 33 PDec & 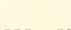 & (5) & $2 \infty$ & 13 & $+\cdots$ & 207 & 269 & 619 & $-\infty$ & 231 & 510 & 1,791 & 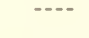 & 1,061 & 4,263 & \\
\hline 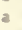 & SBddEZ &.- & $\ldots$ & $\begin{array}{l}\hat{u} \\
0\end{array}$ & 2 &.-- & 1 & 3 & 4 & --- & 3 & 9 & 32 & $\ldots$ & 9 & 60 & 289 \\
\hline$a$ & ODOE & $\ldots \quad-$ & $\ldots$ & $\begin{array}{l}0 \\
0\end{array}$ & 3 & $\cdots$ & 1 & 3 & 4 & $=-$. & 3 & 9 & 32 &.-- & 9 & 60 & 289 \\
\hline a & A3DdFs & & 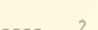 & & 3 & $-\cdots$ & 1 & 3 & 4 & $\cdots$ & 3 & 9 & 32 & $\ldots$. & 9 & 60 & 289 \\
\hline 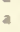 & Bb DDEe & $\cdots$ & $\ldots$ & $\begin{array}{l}1 \\
1\end{array}$ & $\begin{array}{l}0 \\
0\end{array}$ & --- & 5 & 11 & 18 & $-\cdots$ & 12 & 39 & 145 & $\ldots$ & 36 & 260 & 128 \\
\hline a & B’DJE $E$ & $\ldots$ & .... & 1 & $\begin{array}{l}0 \\
0\end{array}$ & $-\ldots$ & 5 & 11 & 18 & $\ldots$ & 12 & 39 & 145 & $-\cdots$ & 36 & 260 & $\begin{array}{l}128 \\
128\end{array}$ \\
\hline \& & Bbddee. & n- & $-20-0$ & & 0 & $\cdots$ & 5 & 11 & 18 & ...- & 12 & 39 & 145 & $\cdots$ & 36 & 260 & 128 \\
\hline A & sbDee. & $\ldots$ & $\ldots$ & $\begin{array}{l}55 \\
55\end{array}$ & $\begin{array}{l}57 \\
57\end{array}$ & 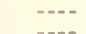 & 205 & 653 & 1,954 & -... & 225 & 1,051 & 6,352 & -... & 1,044 & 9,863 & 78,026 \\
\hline A & bbddEE & --- & $\ldots$ & $\begin{array}{l}53 \\
55\end{array}$ & $\begin{array}{l}57 \\
57\end{array}$ & $\ldots$ & 205 & 653 & 1,954 & --- & 225 & 1,051 & 6,352 & $\ldots$ & 1,044 & 9,863 & 78,026 \\
\hline a & 330den & 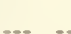 & 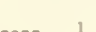 & & 37 & $\cdots$ & 205 & 653 & 1,954 & $-\infty$ & 225 & 1,051 & 6,352 & $-\cdots$ & 1,044 & 9,863 & 78,026 \\
\hline a & Baddre. & $\ldots$ & 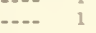 & $\begin{array}{l}1 \\
1\end{array}$ & $\begin{array}{l}1 \\
1\end{array}$ & $\ldots$ & 3 & 14 & 51 & $\ldots$ & 6 & 46 & 333 & .... & 17 & 334 & 3,310 \\
\hline a & Sbicee & $\ldots-\ldots$ & -... & 1 & $\begin{array}{l}1 \\
1\end{array}$ &..- & 3 & 14 & 51 & 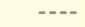 & 6 & 46 & 333 & $\cdots$ & 17 & 334 & 3,310 \\
\hline a & BO dटाएE & -- & -... & $i$ & $\begin{array}{l}1 \\
1\end{array}$ &..- & 3 & 14 & 51 & $\ldots$ & 6 & 46 & 333 & $\cdots$ & 17 & 334 & 3,310 \\
\hline a & SSDDRe &.- & -... & 1 & $\begin{array}{l}1 \\
1\end{array}$ & $\cdots$ & 3 & 14 & 51 & $-\cdot-$ & 6 & 46 & 333 & $-\ldots$ & 17 & 334 & 3,310 \\
\hline a & BbDdEE & $---\quad-$ & --- & 1 & $\begin{array}{l}1 \\
1\end{array}$ & -... & $\begin{array}{l}3 \\
3\end{array}$ & $\begin{array}{l}14 \\
14\end{array}$ & $\begin{array}{l}51 \\
51\end{array}$ & $\ldots$ & $\begin{array}{l}6 \\
6\end{array}$ & $\begin{array}{l}46 \\
46\end{array}$ & $\begin{array}{l}333 \\
333\end{array}$ & $\ldots$ & $\begin{array}{l}17 \\
17\end{array}$ & $\begin{array}{l}334 \\
334\end{array}$ & $\begin{array}{l}3,310 \\
3,310\end{array}$ \\
\hline a & Bbides & $\ldots$ & $005-169$ & 62 & 28 & & & & & & & 40 & 333 & $\cdots$ & 17 & & 3,310 \\
\hline & Dodare & & $495 \quad 107$ & & 28 & 990 & 698 & 658 & 917 & 990 & 861 & 1,293 & 3,961 & 1,980 & 3,565 & 10,425 & 41,623 \\
\hline a & E.jd dee & -- & --- & 1 & 3 & .... & 1 & 20 & 152 & $\ldots$ & 3 & 60 & 876 & .... & 9 & 461 & 9,489 \\
\hline a & bbDLee & $\ldots$ & -...- & 1 & 3 & -..- & 1 & 20 & 152 & -... & 3 & 60 & 876 & -... & 9 & 461 & 9,489 \\
\hline & boddZP & $\cdots$ & -..- & 1 & 3 & -..- & 1 & 20 & 152 & $\cdots$ & 3 & 60 & 876 & $\ldots$ & 9 & 461 & 9,489 \\
\hline A & obddee & --- & 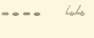 & 146 & $3: 3$ & --- & 203 & 1,794 & 10,962 & -... & 223 & 2,661 & 29,361 & $\ldots$ & 1,035 & 26,392 & 398,638 \\
\hline 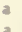 & BbDcee & --- & $\ldots 132$ & 132 & 126 & $\ldots$ & 611 & 1,547 & 4,226 & $\ldots$ & 671 & 2,520 & 13,827 & $\ldots$ & 3,114 & 23,322 & 168,505 \\
\hline a & BbddEe & $-\infty$ & $\ldots 132$ & 132 & 124 & -...- & 611 & 1,547 & 4,226 & $\ldots$ & 671 & 2,520 & 13,827 & -..- & 3,114 & 23,322 & 168,505 \\
\hline a & DSDdRe & $---\quad-$ & $\ldots 132$ & 132 & 124 & $\ldots$ & 611 & 1,547 & 4,226 & $\ldots$ & 671 & 2,520 & 13,827 & $-\ldots$ & 3,114 & 23,322 & 168,505 \\
\hline a & 3ోdcee & -- & $=-132$ & 34 & 677 & -... & 609 & 4,203 & 23,573 & --- & 665 & 6,254 & 63,322 & ... & 3,097 & 61,725 & 856,318 \\
\hline a & sbDdee & $\ldots$ & $\ldots 132$ & 344 & 677 & $\ldots$ & 609 & 4,203 & 23,573 & $\ldots$ & 665 & 6,254 & 63,322 &.-- & 3,097 & 61,725 & 856,318 \\
\hline a & boddze. & $-\cdots$ & $\ldots 132$ & 346 & 677 & $\ldots$ & 609 & 4,203 & 23,573 & $\ldots$ & 665 & 6,254 & 63,322 & $-\cdots$ & 3,097 & 61,725 & 856,318 \\
\hline a & biddee & 4,900 & 10,568 & 19,615 & 37,990 & 19,502 & 64,957 & 220,209 & 906,583 & 9,801 & 35,666 & 159,577 & $1,138,900$ & 39,204 & 247,807 & $2,024,255$ & $19,228,432$ \\
\hline 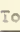 & eal $\ldots \ldots$ & $=5,940$ & 011,955 & 21,525 & 40,967 & 21,632 & 71,150 & 243,078 & $1,010,071$ & 11,881 & 42,786 & 195,771 & $1,435,022$ & 43,264 & 279,555 & $2,367,239$ & $23,103,616$ \\
\hline
\end{tabular}




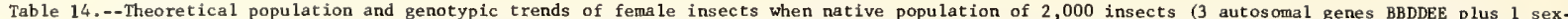
linked gene AA; female is heterogametic) is overflooded by 198,000 or 396,000 partially sterile insects (aabbddee and a-bbddee) during parental, $\mathrm{F}_{1}, \mathrm{~F}_{2}$, and $\mathrm{F}_{3}$ generations. Matings between partially sterile insects are assumed to yield no offspring--Continued

\begin{tabular}{|c|c|c|c|c|c|c|c|c|c|c|c|c|c|c|c|c|}
\hline \multirow[t]{2}{*}{ Genotype } & \multicolumn{4}{|c|}{$\begin{array}{l}90 \text { percent, } \\
\text { fivefold }\end{array}$} & \multicolumn{4}{|c|}{$\begin{array}{l}80 \text { percent, } \\
\text { fivefold }\end{array}$} & \multicolumn{4}{|c|}{$\begin{array}{l}90 \text { percent, } \\
\text { tenfold }\end{array}$} & \multicolumn{4}{|c|}{$\begin{array}{c}80 \text { percent, } \\
\text { tenfold }\end{array}$} \\
\hline & $F_{1}$ & $\mathrm{~F}_{2}$ & $F_{3}$ & $\mathrm{~F}_{4}$ & $\mathrm{~F}_{1}$ & $F_{2}$ & $\mathrm{~F}_{3}$ & $\mathrm{~F}_{4}$ & $\mathrm{~F}_{1}$ & $\mathrm{~F}_{2}$ & $F_{3}$ & $\mathrm{~F}_{4}$ & $F_{1}$ & $\mathrm{~F}_{2}$ & $\mathrm{~F}_{3}$ & $\mathrm{~F}_{4}$ \\
\hline A BBDDEE--- & 25 & 0 & 0 & 0 & 25 & 1 & 0 & 0 & 50 & 2 & 0 & 0 & so & 6 & 2 & 1 \\
\hline A BBDDEe--- & --.- & 0 & 0 & 0 & --.-- & 1 & 0 & 0 & .... & 4 & 1 & 1 & --- & 10 & 9 & 9 \\
\hline A BBDdEE--- & ---- & 0 & 0 & 0 & $-\cdots$ & 1 & 0 & 0 & $\cdots$ & 4 & 1 & 1 & 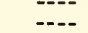 & 10 & 9 & 9 \\
\hline A BbDDEE--- & --.- & 0 & 0 & 0 & $\cdots$ & 1 & 0 & 0 & $\cdots$ & 4 & 1 & 1 & $-\cdots$ & 10 & 9 & 9 \\
\hline A BBDDee--- & -..- & 0 & 0 & 0 & -..- & 1 & 0 & 0 & ---- & 1 & 1 & 2 & ---- & 4 & 11 & 22 \\
\hline $\begin{array}{l}\text { A BBddEE--- } \\
\text { A bbDDEE--- }\end{array}$ & ---- & 0 & 0 & 0 &.-- & 1 & 0 & 0 & --- & 1 & $i$ & 2 & $\cdots$ & 4 & 11 & 22 \\
\hline & $\cdots$ & 0 & 0 & 0 & $\cdots$ & 1 & 0 & 0 & ---- & 1 & 1 & 2 & --- & 4 & 11 & 22 \\
\hline A BBDdEe--- & -... & 1 & 0 & 0 & ....- & 3 & 2 & 1 & -..- & 6 & 7 & 10 & -... & 19 & 45 & 95 \\
\hline A BbDDEe--- & --- & 1 & 0 & 0 & --- & 3 & 2 & 1 & --- & 6 & 7 & 10 &..- & 19 & 45 & 95 \\
\hline A BbDdEE--- & --.- & 1 & 0 & 0 & ---- & 3 & 2 & 1 & ---- & 6 & 7 & 10 &.-- & 19 & 45 & 95 \\
\hline A BBDDEE--- & --- & 0 & 0 & 0 & --- & 1 & 2 & 4 & ---- & 3 & 8 & 24 & -..- & 9 & 59 & 260 \\
\hline A 8 BddEe--- & ---- & 0 & 0 & 0 & --.- & 1 & 2 & 4 & ---- & 3 & 8 & 24 & -... & 9 & 59 & 260 \\
\hline A BbDDee--- & ---- & 0 & 0 & 0 & --- & 1 & 2 & 4 & ---- & 3 & 8 & 24 & --- & 9 & 59 & 260 \\
\hline A BbddEE--- & ---- & 0 & 0 & 0 & --.- & 1 & 2 & 4 & -..- & 3 & 8 & 24 &..- & 9 & 59 & 260 \\
\hline A bbDDEe--- & ---- & 0 & 0 & 0 & ---- & 1 & 2 & 4 & ---- & 3 & 8 & 24 & --.- & 9 & 59 & 260 \\
\hline A bbDdEE--- & -..- & 0 & 0 & 0 & --- & 1 & 2 & 4 & $\cdots$ & 3 & 8 & 24 & --- & 9 & 59 & 260 \\
\hline a BBDDEE--- & ---- & 0 & 0 & 0 & --.- & 1 & 0 & 0 & ---- & 2 & 1 & 0 & ---- & 5 & 4 & 4 \\
\hline A BbDdEe--- & 497 & 64 & 10 & 2 & 995 & 252 & 103 & 68 & 995 & 326 & 210 & 302 & 1.990 & 1,297 & 1,701 & 3,347 \\
\hline a BBDDEe--- & .... & 0 & 0 & 0 & -.--- & 1 & 1 & 1 & -.-- & 3 & 3 & 4 & -..-- & 9 & 22 & 44 \\
\hline a BBDdEE--- &.-- & 0 & 0 & 0 & --- & 1 & 1 & 1 & $\cdots$ & 3 & 3 & 4 &.-- & 9 & 22 & 44 \\
\hline a BbDDEE--- & --.- & 0 & 0 & 0 & -... & 1 & 1 & 1 & ---- & 3 & 3 & 4 & $-\cdots$ & 9 & 22 & 44 \\
\hline A BBddee--- & -... & 0 & 0 & 0 & -..-- & 1 & 3 & 12 & ---- & 1 & 10 & 68 & --.- & 4 & 82 & 774 \\
\hline A bbDDee--- & $-\ldots$ & 0 & 0 & 0 &.--- & 1 & 3 & 12 & $-\ldots$ & 1 & 10 & 68 & --- & 4 & 82 & 774 \\
\hline A bbddEE--- & $--\cdot-$ & 0 & 0 & 0 & $\cdots$ & 1 & 3 & 12 & --- & 1 & 10 & 68 & -.-- & 4 & 82 & 774 \\
\hline A BbDdee--- & -..- & 45 & 20 & 9 & ---- & 208 & 236 & 317 &.--- & 229 & 396 & 1,094 & --- & 1,066 & 3,698 & 13,780 \\
\hline A 8bddEe--- & ---- & 45 & 20 & 9 & ---- & 208 & 236 & 317 & ---- & 229 & 396 & 1,094 & --- & 1,066 & 3,698 & 13,780 \\
\hline A bbDaEe--- & ---- & 45 & 20 & 9 & --- & 208 & 236 & 317 & ---- & 229 & 396 & 1,094 & -..- & 1,066 & 3,698 & 13,780 \\
\hline a BBDDee--- & --.- & 0 & 0 & 0 & -..- & 1 & 1 & 2 & -... & 1 & 4 & 11 & ---- & 4 & 29 & 124 \\
\hline a BBddEE--- & --.- & 0 & 0 & 0 & ---- & 1 & 1 & 2 & --- & 1 & 4 & 11 & --- & 4 & 29 & 124 \\
\hline a bbDDEE--- & -... & 0 & 0 & 0 & -... & 1 & 1 & 2 & ---- & 1 & 4 & 11 & --- & 4 & 29 & 124 \\
\hline a BBDdEe--- & --.- & 1 & 0 & 0 & --- & 3 & 5 & 8 & ---- & 6 & 16 & 50 & -..- & 18 & 120 & 526 \\
\hline a $8 \mathrm{bDDEe}---$ & --.- & 1 & 0 & 0 & -.-- & 3 & 5 & 8 & ---- & 6 & 16 & 50 & -- & 18 & 120 & 526 \\
\hline a BbDdEE --- & -..- & 1 & 0 & 0 & -..- & 3 & 5 & 8 & --- & 6 & 16 & 50 & -... & 18 & 120 & 526 \\
\hline A 8bddee---- & -... & 45 & 51 & 48 &..-- & 206 & 632 & 1,759 &..-- & 226 & 961 & 5,061 & --- & 1,058 & 9,626 & 70,101 \\
\hline A bbDdee--- & ---- & 45 & 51 & 48 & ---- & 206 & 632 & 1,759 & --- & 226 & 961 & 5,061 & --- & 1,058 & 9,626 & 70,101 \\
\hline A bbddEe-.- & --.- & 45 & 51 & 48 & ---- & 206 & 632 & 1,759 & $-\cdots$ & 226 & 961 & 5,061 & --- & 1,058 & 9,626 & 70,101 \\
\hline a 8BDdee--- & ---- & 0 & 1 & 0 & -..-- & 1 & 7 & 23 & ---- & 3 & 21 & 137 & -... & 9 & 166 & 1,560 \\
\hline a B8ddEe --- & --.- & 0 & 1 & 0 & --- & 1 & 7 & 23 & ---- & 3 & 21 & 137 & --- & 9 & 16 & 1,560 \\
\hline a BbDDee -.-- & --.-- & 0 & 1 & 0 & ---- & 1 & 7 & 23 & ---- & 3 & 21 & 137 & ---- & 9 & 16 & 1,560 \\
\hline a BbddEE_-.- & -...- & 0 & 1 & 0 & -... & 1 & 7 & 23 & --- & 3 & 21 & 137 &.-- & 9 & 166 & 1,560 \\
\hline a bbDDEe--- & ---- & 0 & 1 & 0 & --.- & 1 & 7 & 23 & ---- & 3 & 21 & 137 & --- & 9 & 166 & 1,560 \\
\hline a bbDdEE_--- & -..- & 0 & 1 & 0 &.-- & 1 & 7 & 23 & --- & 3 & 21 & 137 & --- & 9 & 166 & 1,560 \\
\hline a 8bDdEe_--- & 497 & 152 & 49 & 19 & 995 & 662 & 565 & 686 & 994 & 773 & 969 & 390 & 1,990 & 3,394 & 8,858 & 29,854 \\
\hline a B8ddee--- & ---- & 0 & 1 & 1 & -..-- & 1 & 10 & 76 & --.- & 1 & 30 & 412 & $\ldots$ & 4 & 238 & 4,918 \\
\hline a bbDDee --- & --. & 0 & 1 & 1 & ---- & 1 & 10 & 76 & ---- & 1 & 30 & 412 & ---- & 4 & 238 & 4,918 \\
\hline a bbddEE --- & --.- & 0 & 1 & 1 & --- & 1 & 10 & 76 & -.- & 1 & 30 & 412 & $\cdots$ & 4 & 238 & 4,918 \\
\hline A bbddee--- & -..- & 44 & 143 & 294 & ---- & 206 & 1,812 & 10,963 & ---- & 225 & 2,638 & 28,243 & -..- & 1,053 & 27,220 & 41,411 \\
\hline a BbDdee --- & -... & 133 & 121 & 103 & -.-. & 618 & 1,486 & 3,789 & --.- & 676 & 2,276 & 10,940 & ---- & 3,164 & 22,618 & 150,861 \\
\hline a BbddEe--- & ---- & 133 & 121 & 103 &.-- & 618 & 1,486 & 3,789 & --- & 676 & & & ---- & 3,164 & 22,618 & 150,861 \\
\hline a bbDdEe --- & ---- & 133 & 121 & 103 & ---- & 618 & 1,486 & 3,789 & ---- & 676 & 2,276 & 10,940 & --- & 3,164 & 22,618 & 150,861 \\
\hline a Bbddee --- & --.- & 133 & 336 & 633 & -.-- & 617 & 4,235 & 23,534 & ---- & 674 & 6,178 & 60,722 & ---- & 3,155 & 63,588 & 888,475 \\
\hline a bbDdee --- & ---- & 133 & 336 & 633 & ---- & 617 & 4,235 & 23,534 & ---- & 674 & 6,178 & 60,722 & ---- & 3,155 & 63,588 & 888,475 \\
\hline a bbddEe --- & ---- & 133 & 336 & 633 & --- & 617 & 4,235 & 23,534 & --- & 674 & 6,178 & 60,722 & --- & 3,155 & 63,588 & 888,475 \\
\hline a bbddee --9 & .850 & 21,178 & 38,809 & 73,759 & 39,401 & 130,957 & 443,405 & $1,823,495$ & 19,700 & 71,678 & 316,004 & $2,225,268$ & 78,802 & 502,345 & $4,153,020$ & $39,942,048$ \\
\hline Total---- 10 , & 1,870 & 22,518 & 40,605 & 76,459 & 41,416 & 137,075 & 465,776 & $1,923,866$ & 21,741 & 78,530 & 349,646 & $2,492,301$ & 82,832 & 533,756 & $4,492,413$ & $43,789,456$ \\
\hline
\end{tabular}




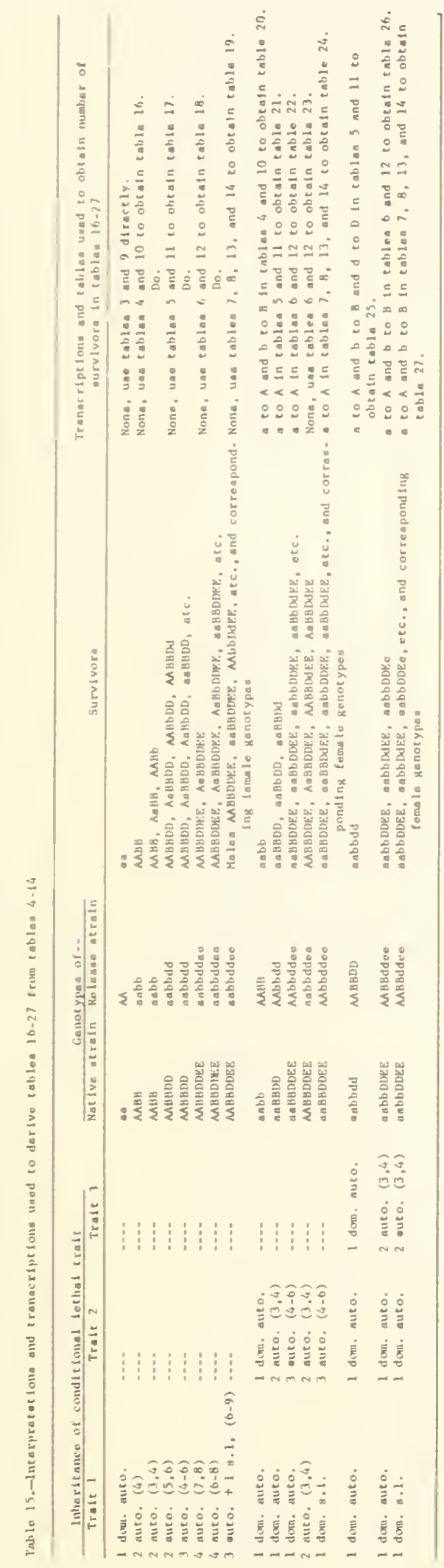


Table 16.--Numbers in each generation in which conditional lethal traits cannot be expressed when native population of 2,000 insects (AABB) was overflooded in parental, $F_{1}, F_{2}$, and $F_{3}$ generations by partially sterile insects (aabb) with 1 conditional lethal trait

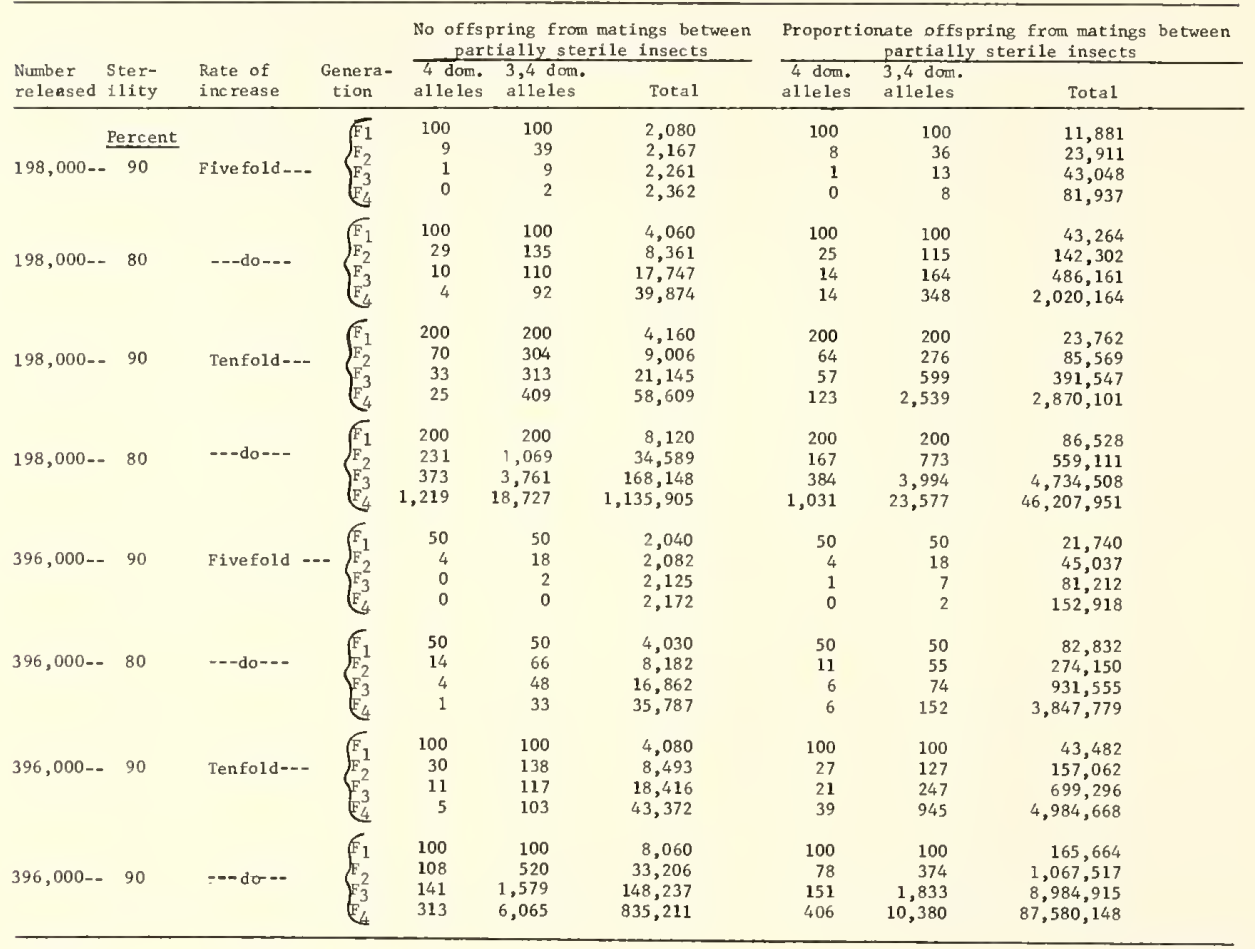

Table 17.--Numbers in each generation in which conditional lethal traits cannot be expressed when native population of 2,000 insects (AABBDD) was overflooded in parental, $F_{1}, F_{2}$, and $F_{3}$ generations by partially sterile insects (aabbdd) with 1 conditional lethal trait

\begin{tabular}{|c|c|c|c|c|c|c|c|c|c|}
\hline \multirow[b]{2}{*}{$\begin{array}{l}\text { Number } \\
\text { released }\end{array}$} & \multirow[b]{2}{*}{$\begin{array}{l}\text { Ster- } \\
\text { ility }\end{array}$} & \multirow[b]{2}{*}{$\begin{array}{l}\text { Rate of } \\
\text { increase }\end{array}$} & \multirow[b]{2}{*}{$\begin{array}{l}\text { Genera- } \\
\text { tion }\end{array}$} & \multicolumn{3}{|c|}{$\begin{array}{l}\text { No offspring from matings be- } \\
\text { tween partially sterile insects }\end{array}$} & \multicolumn{3}{|c|}{$\begin{array}{l}\text { Proportionate offspring from matings } \\
\text { between partially sterile insects }\end{array}$} \\
\hline & & & & $\begin{array}{l}5,6 \text { dom. } \\
\text { alleles }\end{array}$ & $\begin{array}{l}4-6 \text { dom. } \\
\text { alleles. }\end{array}$ & Total & $\begin{array}{l}, 6 \text { dom. } \\
\text { aileles }\end{array}$ & $\begin{array}{l}4-6 \text { dom: } \\
\text { alleles }\end{array}$ & Total \\
\hline \multicolumn{3}{|c|}{ Percent } & \multirow{4}{*}{$\begin{array}{l}F_{1} \\
F_{2} \\
E_{3} \\
F_{4}\end{array}$} & 100 & 100 & 2,080 & 100 & 100 & 11,881 \\
\hline $198,000=-$ & 90 & Five fold.-- & & 16 & 42 & 2,166 & 15 & 41 & 23,911 \\
\hline & 50 & Five $2010=$ & & 1 & 7 & 2,260 & 2 & 12 & 43,050 \\
\hline & & & & 0 & 1 & 2,362 & 0 & 4 & 81,936 \\
\hline \multirow{4}{*}{$198,000--$} & \multirow{4}{*}{80} & \multirow{4}{*}{--do-- } & & 100 & 100 & 4,060 & 100 & 100 & 43,264 \\
\hline & & & & 52 & 151 & 8,365 & 44 & 119 & 142,301 \\
\hline & & & & 14 & 90 & 17,747 & 20 & 132 & 486,161 \\
\hline & & & $\mathrm{E}_{4}$ & 4 & 51 & 39,873 & 12 & 174 & $2,020,176$ \\
\hline \multirow{4}{*}{$198,000=-$} & \multirow{4}{*}{90} & \multirow{4}{*}{ Tenfold...- } & & 200 & 200 & 4,160 & 200 & 200 & 23,762 \\
\hline & & & & 126 & 337 & 9,005 & 114 & 307 & 85,571 \\
\hline & & & & 50 & 276 & 21,144 & 83 & 507 & 391,548 \\
\hline & & & $\left(F_{4}\right.$ & 29 & 269 & 58,607 & 124 & 1,442 & $2,870,116$ \\
\hline \multirow{4}{*}{$198,000=-$} & \multirow{4}{*}{80} & \multirow{4}{*}{$--\mathrm{do}^{--}$} & & 200 & 200 & 8,120 & 200 & 200 & 86,528 \\
\hline & & & & 412 & 1,183 & 34,399 & 298 & 851 & 559,111 \\
\hline & & & & 551 & 3,238 & 168,148 & 474 & 3,196 & $4,734,505$ \\
\hline & & & & 1,483 & 12,901 & $1,135,912$ & 939 & 12,326 & $46,207,856$ \\
\hline \multirow{4}{*}{$396,000--$} & \multirow{4}{*}{90} & \multirow{4}{*}{ Fivefold--- } & & 50 & 50 & 2,040 & 50 & 50 & 21,740 \\
\hline & & & & 7 & 19 & 2,082 & 6 & 18 & 45,036 \\
\hline & & & & 0 & 3 & 2,126 & 1 & 5 & 81,210 \\
\hline & & & & 0 & 0 & 2,171 & 0 & 0 & 152,920 \\
\hline \multirow{4}{*}{$396,000--$} & \multirow{4}{*}{80} & \multirow{4}{*}{--do.- } & & 50 & 50 & 4,030 & 50 & 50 & 82,832 \\
\hline & & & 22 & 24 & 72 & 8,182 & 20 & 61 & 274,150 \\
\hline & & & & 5 & 38 & 16,861 & 8 & 58 & 931,557 \\
\hline & & & $\mathrm{F}_{4}$ & 1 & 16 & 35,788 & 4 & 70 & $3,847,777$ \\
\hline \multirow{4}{*}{$396,000=-$} & \multirow{4}{*}{90} & \multirow{4}{*}{ Tenfold--- } & F & 101 & 101 & 4,081 & 101 & 101 & 43,482 \\
\hline & & & $F_{2}^{1}$ & 54 & 154 & 8,494 & 49 & 140 & 157,060 \\
\hline & & & & 15 & 95 & 18,414 & 29 & 197 & 699,296 \\
\hline & & & $\mathrm{E}_{\mathrm{L}}^{3}$ & 5 & 58 & 43,374 & 34 & 472 & $4,984,654$ \\
\hline \multirow{4}{*}{$396,000--$} & & & $F_{1}$ & 101 & 101 & 8,060 & 101 & 101 & 165,663 \\
\hline & 80 & --dom- & $E_{2}^{1}$ & 192 & 574 & 33,206 & 138 & 413 & $1,067,514$ \\
\hline & & & & 193 & 1,089 & 148,237 & 194 & 1,423 & $8,984,898$ \\
\hline & & & $\mathrm{E}_{4}^{3}$ & 326 & 3,589 & 835,208 & 326 & 4,922 & $87,579,904$ \\
\hline
\end{tabular}


Fabie 18...-itubers in eac. seneration $2 n$ which conditional lethal traits cannot be expressed when native population of 2. 0 asects AASSODEE) was overtlooded in parenta1, $F_{1}, F_{2}$, and $F_{3}$ generations by partially sterile insects abbddee' with l conditional lethal trait

\begin{tabular}{|c|c|c|c|c|c|c|c|c|c|}
\hline \multirow[b]{2}{*}{$\begin{array}{l}\text { Sivber } \\
\text { released }\end{array}$} & \multirow[b]{2}{*}{$\begin{array}{l}\text { Ster }- \\
212 t\end{array}$} & \multirow[b]{2}{*}{$\begin{array}{l}\text { Sate of } \\
\text { increase }\end{array}$} & \multirow[b]{2}{*}{$\begin{array}{l}\text { Genera- } \\
\text { tion }\end{array}$} & \multicolumn{3}{|c|}{$\begin{array}{c}\text { No offsfring from matings between } \\
\text { partially sterile insects }\end{array}$} & \multicolumn{3}{|c|}{$\begin{array}{c}\text { Proportionate offspzing from matings between } \\
\text { partially sterile insects }\end{array}$} \\
\hline & & & & $\begin{array}{l}7,8 \text { doo. } \\
\text { sileles }\end{array}$ & $\begin{array}{l}\text { 6-8 don. } \\
\text { slleles }\end{array}$ & Total & $\begin{array}{l}7,8 \text { don. } \\
\text { alleles }\end{array}$ & $\begin{array}{l}6-8 \text { dom. } \\
\text { alleles }\end{array}$ & Total \\
\hline$: 20 . .$. & $\frac{\text { Percent }}{77}$ & Fivefold-.. & & $\begin{array}{r}100 \\
7 \\
0 \\
0\end{array}$ & $\begin{array}{r}100 \\
21 \\
1 \\
0\end{array}$ & $\begin{array}{l}2,080 \\
?, 166 \\
, 260 \\
2,36=\end{array}$ & $\begin{array}{r}100 \\
6 \\
0 \\
0\end{array}$ & $\begin{array}{r}100 \\
20 \\
2 \\
0\end{array}$ & $\begin{array}{l}11,881 \\
23,911 \\
43,050 \\
81,935\end{array}$ \\
\hline 208,70 & 36 & - -do.. & & $\begin{array}{r}100 \\
20 \\
2 \\
0\end{array}$ & $\begin{array}{r}100 \\
70 \\
15 \\
3\end{array}$ & $\begin{array}{r}1,060 \\
8,365 \\
17,747 \\
39,873\end{array}$ & $\begin{array}{r}100 \\
17 \\
2 \\
1\end{array}$ & $\begin{array}{r}100 \\
59 \\
70 \\
8\end{array}$ & $\begin{array}{r}43,264 \\
142,301 \\
486,159 \\
2,020,161\end{array}$ \\
\hline$: 03,10$. & $\nu$ & Eenfold... & & $\begin{array}{r}710 \\
54 \\
9 \\
2\end{array}$ & $\begin{array}{r}200 \\
168 \\
59 \\
22\end{array}$ & $\begin{array}{r}., 160 \\
9,005 \\
21,143 \\
58,606\end{array}$ & $\begin{array}{r}200 \\
50 \\
13 \\
8\end{array}$ & $\begin{array}{r}200 \\
153 \\
92 \\
100\end{array}$ & $\begin{array}{r}23,762 \\
85,571 \\
391,545 \\
2,870,090\end{array}$ \\
\hline $198,00=$ & e & - dom. & & $\begin{array}{r}200 \\
158 \\
93 \\
157\end{array}$ & $\begin{array}{r}200 \\
548 \\
626 \\
1.415\end{array}$ & $\begin{array}{r}8,120 \\
24,399 \\
168,147 \\
1,135,904\end{array}$ & $\begin{array}{r}200 \\
114 \\
62 \\
44\end{array}$ & $\begin{array}{l}200 \\
397 \\
499 \\
688\end{array}$ & $\begin{array}{r}86,528 \\
559,110 \\
4,734,500 \\
46,207,866\end{array}$ \\
\hline $175, \quad=-$ & 9 & Fiverold =- & & $\begin{array}{r}50 \\
3 \\
1 \\
0\end{array}$ & $\begin{array}{r}\text { So } \\
9 \\
0 \\
0\end{array}$ & $\begin{array}{l}2,040 \\
?, 082 \\
2,126 \\
2,171\end{array}$ & $\begin{array}{r}50 \\
2 \\
0 \\
0\end{array}$ & $\begin{array}{r}50 \\
9 \\
1 \\
0\end{array}$ & $\begin{array}{r}21,741 \\
45,036 \\
81,209 \\
152,919\end{array}$ \\
\hline 395,1000 & 8 & $-40-+$ & & $\begin{array}{r}50 \\
9 \\
1 \\
0\end{array}$ & $\begin{array}{r}50 \\
32 \\
5 \\
1\end{array}$ & $\begin{array}{r}4,030 \\
8,18 ? \\
16,861 \\
35,788\end{array}$ & $\begin{array}{r}50 \\
7 \\
1 \\
0\end{array}$ & $\begin{array}{r}50 \\
27 \\
8 \\
2\end{array}$ & $\begin{array}{r}82,832 \\
274,150 \\
931,556 \\
3,847,774\end{array}$ \\
\hline $120,1 \times-$. & 90 & entold--- & & $\begin{array}{r}101 \\
20 \\
2 \\
0\end{array}$ & $\begin{array}{r}101 \\
71 \\
16 \\
3\end{array}$ & $\begin{array}{r}4,080 \\
8,494 \\
18,414 \\
43,374\end{array}$ & $\begin{array}{r}101 \\
19 \\
4 \\
1\end{array}$ & $\begin{array}{r}101 \\
65 \\
30 \\
21\end{array}$ & $\begin{array}{r}43,482 \\
157,060 \\
699,296 \\
4,984,656\end{array}$ \\
\hline $396,-732=$ & 80 & - do =- & ? & $\begin{array}{r}101 \\
68 \\
24 \\
17\end{array}$ & $\begin{array}{l}101 \\
254 \\
202 \\
224\end{array}$ & $\begin{array}{r}8,050 \\
33,206 \\
148,238 \\
335,210\end{array}$ & $\begin{array}{r}101 \\
49 \\
21 \\
12\end{array}$ & $\begin{array}{l}101 \\
183 \\
192 \\
209\end{array}$ & $\begin{array}{r}165,664 \\
1,067,514 \\
8,984,906 \\
87,580,048\end{array}$ \\
\hline
\end{tabular}

-able 17.--Mebers in each generation in which conditions lethal traits cannot be expressed when native population of 2,000 1nsects ( 3 autosonal genes B:DDEE plus 1 sex-linked gene $\mathrm{AA}$; female is heteroganet1c) was overflooded in parental. $F_{1}, F_{2}$, and $F_{3}$ generations by partially sterile insects (aabbddee, a-bbddee) with 1 condiclonal lechal iralt 2

\begin{tabular}{|c|c|c|c|c|c|c|c|c|c|}
\hline \multirow{2}{*}{$\begin{array}{l}\text { rumber } \\
\text { released }\end{array}$} & \multirow[t]{2}{*}{ Stertlity } & \multirow{2}{*}{$\begin{array}{l}\text { Pace of } \\
\text { increase }\end{array}$} & \multirow{2}{*}{$\begin{array}{l}\text { Cenera- } \\
\text { tion }\end{array}$} & \multicolumn{3}{|c|}{$\begin{array}{c}\text { No offspring from matings between } \\
\text { partially sterile insects }\end{array}$} & \multicolumn{3}{|c|}{$\begin{array}{l}\text { Proportionate offspring frow matings } \\
\text { between partially sterile insects }\end{array}$} \\
\hline & & & & Nolos & Females & Total & Males & Females & Total \\
\hline $198, \cdots$ & $\frac{\text { Perce t }}{9}$ & Flvefold-.. & & $\begin{array}{r}1,040 \\
139 \\
13 \\
2\end{array}$ & $\begin{array}{r}545 \\
76 \\
7 \\
1\end{array}$ & $\begin{array}{l}2,080 \\
2,166 \\
2,322 \\
2,772\end{array}$ & $\begin{array}{r}1,040 \\
186 \\
39 \\
7\end{array}$ & $\begin{array}{r}545 \\
99 \\
15 \\
4\end{array}$ & $\begin{array}{l}11,880 \\
23,910 \\
43,050 \\
81,934\end{array}$ \\
\hline $198,000--$ & 80 & --do-- & & $\begin{array}{r}2,030 \\
460 \\
156 \\
70\end{array}$ & $\begin{array}{r}1,040 \\
252 \\
84 \\
42\end{array}$ & $\begin{array}{r}4,060 \\
8,364 \\
18,214 \\
45,974\end{array}$ & $\begin{array}{r}2,030 \\
663 \\
362 \\
350\end{array}$ & $\begin{array}{r}1,040 \\
347 \\
190 \\
184\end{array}$ & $\begin{array}{r}43,264 \\
142,300 \\
486,156 \\
2,020,142\end{array}$ \\
\hline $198,000=-$ & 90 & Tenfold-.. & & $\begin{array}{r}2,080 \\
689 \\
390 \\
342\end{array}$ & $\begin{array}{r}1,090 \\
382 \\
218 \\
190\end{array}$ & $\begin{array}{r}4,160 \\
9,004 \\
21,610 \\
65,288\end{array}$ & $\begin{array}{l}2,080 \\
1,014 \\
1,027 \\
2,342\end{array}$ & $\begin{array}{r}1,090 \\
548 \\
550 \\
1,230\end{array}$ & $\begin{array}{r}23,762 \\
85,572 \\
391,542 \\
2,870,044\end{array}$ \\
\hline $198,000--$ & 80 & --do-. & & $\begin{array}{r}4,060 \\
2,209 \\
3,833 \\
14,649\end{array}$ & $\begin{array}{l}2,080 \\
1,242 \\
2,144 \\
7,997\end{array}$ & $\begin{array}{r}8,120 \\
34,400 \\
171,406 \\
1,206,836\end{array}$ & $\begin{array}{r}4,060 \\
3,515 \\
6,915 \\
20,800\end{array}$ & $\begin{array}{l}2,080 \\
1,855 \\
3,595 \\
4,849\end{array}$ & $\begin{array}{r}86,528 \\
559,110 \\
4,734,478 \\
46,207,232\end{array}$ \\
\hline $396,000 \ldots$ & 90 & Fivefold-..- & & $\begin{array}{r}1,020 \\
92 \\
8 \\
1\end{array}$ & $\begin{array}{r}522 \\
51 \\
4 \\
0\end{array}$ & $\begin{array}{l}2,040 \\
2,082 \\
2,188 \\
2,582\end{array}$ & $\begin{array}{r}1,020 \\
129 \\
19 \\
4\end{array}$ & $\begin{array}{r}522 \\
67 \\
10 \\
2\end{array}$ & $\begin{array}{r}21,740 \\
45,036 \\
81,210 \\
152,918\end{array}$ \\
\hline $396,000--$ & 80 & -rdo-. & & $\begin{array}{r}2,015 \\
355 \\
79 \\
26\end{array}$ & $\begin{array}{r}1,020 \\
183 \\
46 \\
15\end{array}$ & $\begin{array}{r}4,030 \\
8,182 \\
17,344 \\
42,124\end{array}$ & $\begin{array}{r}2,015 \\
539 \\
249 \\
188\end{array}$ & $\begin{array}{r}1,020 \\
274 \\
121 \\
95\end{array}$ & $\begin{array}{r}82,832 \\
274,150 \\
931,552 \\
3,847,732\end{array}$ \\
\hline $396,000 \ldots$ & 90 & Tenfold-..- & & $\begin{array}{r}2,040 \\
467 \\
164 \\
80\end{array}$ & $\begin{array}{r}1,045 \\
242 \\
92 \\
44\end{array}$ & $\begin{array}{r}4,080 \\
8,494 \\
18,898 \\
50,022\end{array}$ & $\begin{array}{r}2,040 \\
724 \\
539 \\
925\end{array}$ & $\begin{array}{r}1,045 \\
381 \\
286 \\
485\end{array}$ & $\begin{array}{r}43,482 \\
157,060 \\
699,292 \\
4,984,602\end{array}$ \\
\hline $396,000=$ & 80 & - dom- & 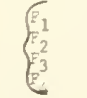 & $\begin{array}{l}4,030 \\
1,592 \\
1,759 \\
2,880\end{array}$ & $\begin{array}{r}2,040 \\
862 \\
961 \\
2,356\end{array}$ & $\begin{array}{r}8,060 \\
33,206 \\
151,844 \\
921,802\end{array}$ & $\begin{array}{r}4,030 \\
2,826 \\
4,330 \\
10,229\end{array}$ & $\begin{array}{l}2,040 \\
1,461 \\
2,256 \\
5,290\end{array}$ & $\begin{array}{r}165,664 \\
1,067,512 \\
8,984,826 \\
87,578,912\end{array}$ \\
\hline
\end{tabular}


Table 20.--Numbers in each generation in which conditional lethal traits cannot be expressed when native population of 2,000 insects (aabb) was overflooded in parental, $F_{1}, F_{2}$, and $F_{3}$ generations by partially sterile insects (AABB) with 2 conditional lethal traits

\begin{tabular}{|c|c|c|c|c|c|c|c|}
\hline \multirow{2}{*}{$\begin{array}{l}\text { Number } \\
\text { released }\end{array}$} & \multirow{2}{*}{ Sterility } & \multirow{2}{*}{$\begin{array}{l}\text { Rate of } \\
\text { increase }\end{array}$} & \multirow{2}{*}{$\begin{array}{l}\text { Genera- } \\
\text { tion }\end{array}$} & \multicolumn{2}{|c|}{$\begin{array}{l}\text { No offspring from matings be- } \\
\text { tween partially sterile insects }\end{array}$} & \multicolumn{2}{|c|}{$\begin{array}{c}\text { Proportionate offspring from matings } \\
\text { between partially sterile insects }\end{array}$} \\
\hline & & & & Survivors & Total offspring & Survivors & Total offspring \\
\hline $198,000--$ & $\frac{\text { Percent }}{90}$ & Fivefold--- & $\left\{\begin{array}{l}F_{1} \\
F_{2} \\
F_{3}^{3} \\
F_{4}\end{array}\right.$ & $\begin{array}{r}100 \\
9 \\
1 \\
0\end{array}$ & $\begin{array}{l}2,080 \\
2,167 \\
2,261 \\
2,362\end{array}$ & $\begin{array}{r}100 \\
8 \\
1 \\
0\end{array}$ & $\begin{array}{l}11,881 \\
23,911 \\
43,048 \\
84,979\end{array}$ \\
\hline $198,000=-$ & 80 & --do.- & $\left\{\begin{array}{l}F_{1} \\
F_{2} \\
F_{3} \\
F_{4}\end{array}\right.$ & $\begin{array}{r}100 \\
29 \\
10 \\
4\end{array}$ & $\begin{array}{r}4,060 \\
8,361 \\
17,747 \\
39,874\end{array}$ & $\begin{array}{r}100 \\
25 \\
14 \\
14\end{array}$ & $\begin{array}{r}43,264 \\
142,302 \\
486,161 \\
2,020,164\end{array}$ \\
\hline $198,000--$ & 90 & Tenfold--- & $\left\{\begin{array}{l}F_{1} \\
F_{2} \\
F_{3} \\
E_{4}\end{array}\right.$ & $\begin{array}{r}200 \\
70 \\
33 \\
25\end{array}$ & $\begin{array}{r}4,160 \\
9,006 \\
21,145 \\
58,609\end{array}$ & $\begin{array}{r}200 \\
64 \\
57 \\
123\end{array}$ & $\begin{array}{r}23,762 \\
85,569 \\
391,547 \\
2,870,101\end{array}$ \\
\hline $198,000--$ & 80 & --do-- & $\left\{\begin{array}{l}F_{1} \\
F_{2} \\
F_{3} \\
F_{4}\end{array}\right.$ & $\begin{array}{r}200 \\
230 \\
373 \\
1,219\end{array}$ & $\begin{array}{r}8,120 \\
34,589 \\
168,148 \\
1,135,905\end{array}$ & $\begin{array}{r}200 \\
167 \\
384 \\
1031\end{array}$ & $\begin{array}{r}86,528 \\
559,111 \\
4,734,508 \\
46,207,951\end{array}$ \\
\hline $396,000=$ & 90 & Five fold ... & $\left\{\begin{array}{l}F_{1} \\
F_{2} \\
F_{3} \\
F_{4}\end{array}\right.$ & $\begin{array}{r}50 \\
4 \\
0 \\
0\end{array}$ & $\begin{array}{l}2,040 \\
2,082 \\
2,125 \\
2,172\end{array}$ & $\begin{array}{r}50 \\
4 \\
0 \\
0\end{array}$ & $\begin{array}{r}21,740 \\
45,037 \\
81,212 \\
152,918\end{array}$ \\
\hline $396,000--$ & 80 & -- do -- & $\left\{\begin{array}{l}F_{1} \\
F_{2} \\
F_{3} \\
F_{4}\end{array}\right.$ & $\begin{array}{r}50 \\
14 \\
4 \\
1\end{array}$ & $\begin{array}{r}4,030 \\
8,182 \\
16,862 \\
35,787\end{array}$ & $\begin{array}{r}50 \\
11 \\
6 \\
6\end{array}$ & $\begin{array}{r}82,832 \\
274,150 \\
931,555 \\
3,847,779\end{array}$ \\
\hline $396,000--$ & 90 & Tenfold =-- & $\left\{\begin{array}{l}F_{1} \\
F_{2} \\
F_{3} \\
F_{4}\end{array}\right.$ & $\begin{array}{r}100 \\
30 \\
11 \\
5\end{array}$ & $\begin{array}{r}4,080 \\
8,493 \\
18,416 \\
43,372\end{array}$ & $\begin{array}{r}100 \\
27 \\
21 \\
39\end{array}$ & $\begin{array}{r}43,482 \\
157,062 \\
699,296 \\
4,984,668\end{array}$ \\
\hline $396,000=-$ & 80 & -do -- & $\left\{\begin{array}{l}F_{1} \\
F_{2} \\
F_{2} \\
F_{4}\end{array}\right.$ & $\begin{array}{l}100 \\
108 \\
141 \\
313\end{array}$ & $\begin{array}{r}8,060 \\
33,206 \\
148,237 \\
835,211\end{array}$ & $\begin{array}{r}100 \\
78 \\
151 \\
406\end{array}$ & $\begin{array}{r}165,664 \\
1,067,517 \\
8,984,915 \\
87,580,148\end{array}$ \\
\hline
\end{tabular}

Table 21.--Numbers in each generation in which conditional lethal traits cannot be expressed when native population of 2,000 insects (aaBBDD) was overflooded in parental, $F_{1}, F_{2}$, and $F_{3}$ generations by partially sterile insects (AAbbdd) with 2 conditional lethal traits

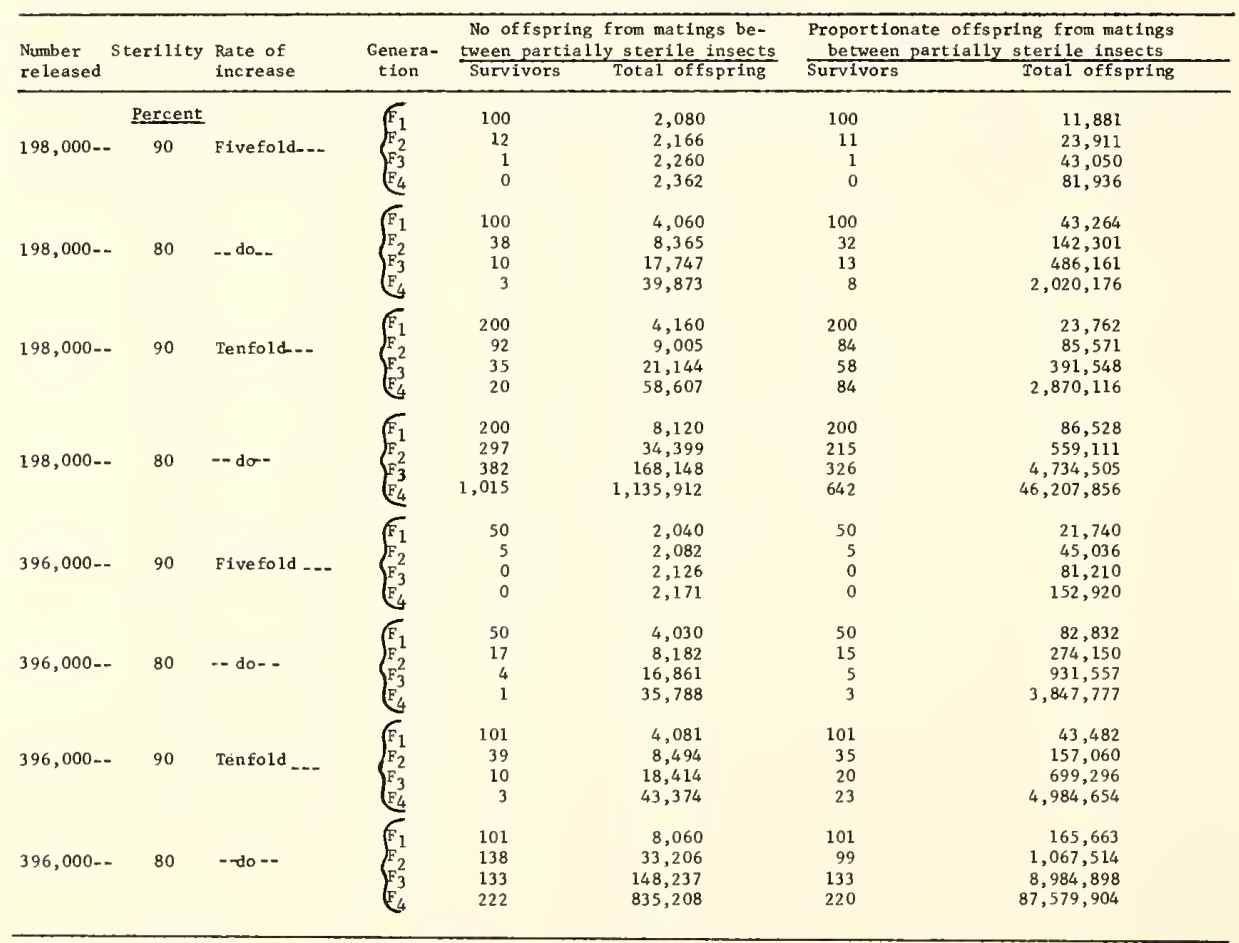


Table 22 .--Numers in each generation in which conditionel lethal traits cannot be expressed when nat lve population of 2.000 insects (aABDDEE) vas overflooded in parental, $F_{1}, F_{2}$, and $F_{3}$ geaerations by partially sterlle insects (Atboddee) with 2 conditional lethal traits

\begin{tabular}{|c|c|c|c|c|c|c|c|}
\hline \multirow{2}{*}{$\begin{array}{l}\text { sumer } \\
\text { relessed }\end{array}$} & \multirow[t]{2}{*}{ Sterility } & \multirow{2}{*}{$\begin{array}{l}\text { Rate of } \\
\text { lacrease }\end{array}$} & \multirow{2}{*}{$\begin{array}{l}\text { Geneta. } \\
\text { tion }\end{array}$} & \multicolumn{2}{|c|}{$\begin{array}{l}\text { No of fspring f nom matings be- } \\
\text { tween partially sterile insects }\end{array}$} & \multicolumn{2}{|c|}{$\begin{array}{l}\text { Proportionate offspring from matings } \\
\text { between partially sterile Insects }\end{array}$} \\
\hline & & & & Survivors & Total of fspring & Survivors & Total offspring \\
\hline $108,000 \ldots$ & $\frac{\text { Pefcent }}{00}$ & Fivefold-... & $\left\{\begin{array}{l}E_{1} \\
E_{3} \\
E_{6}\end{array}\right.$ & $\begin{array}{r}100 \\
10 \\
0 \\
0\end{array}$ & $\begin{array}{l}2,080 \\
2,166 \\
2,260 \\
2,362\end{array}$ & $\begin{array}{r}100 \\
10 \\
0 \\
0\end{array}$ & $\begin{array}{l}11,881 \\
23,911 \\
43,050 \\
81,935\end{array}$ \\
\hline $198,000=-$ & 80 & --do-- & $\left\{\begin{array}{l}E_{1} \\
E_{3} \\
E^{3}\end{array}\right.$ & $\begin{array}{r}100 \\
42 \\
6 \\
0\end{array}$ & $\begin{array}{r}4,060 \\
8,365 \\
17,747 \\
39,873\end{array}$ & $\begin{array}{r}100 \\
36 \\
13 \\
3\end{array}$ & $\begin{array}{r}43,264 \\
142,301 \\
486,159 \\
2,020,161\end{array}$ \\
\hline $198,000=-$ & 90 & Tenfold...- & $\left\{\begin{array}{l}5 \\
F_{2} \\
3 \\
3 \\
0\end{array}\right.$ & $\begin{array}{r}200 \\
103 \\
31 \\
18\end{array}$ & $\begin{array}{r}4,160 \\
9,005 \\
21,163 \\
58,606\end{array}$ & $\begin{array}{r}200 \\
96 \\
52 \\
57\end{array}$ & $\begin{array}{r}23,762 \\
85,571 \\
391,565 \\
2,870,090\end{array}$ \\
\hline $198,000=-$ & 80 & -- do $=-$ & & $\begin{array}{l}200 \\
329 \\
352 \\
784\end{array}$ & $\begin{array}{r}8,120 \\
34,399 \\
168,147 \\
1,135,904\end{array}$ & $\begin{array}{l}200 \\
239 \\
280 \\
376\end{array}$ & $\begin{array}{r}86,528 \\
559,110 \\
4,734,500 \\
46,207,866\end{array}$ \\
\hline $396,000-$. & 90 & Fivefold-... & & $\begin{array}{r}50 \\
6 \\
0 \\
0\end{array}$ & $\begin{array}{l}2,040 \\
2,082 \\
2,126 \\
2,171\end{array}$ & $\begin{array}{r}50 \\
6 \\
0 \\
0\end{array}$ & $\begin{array}{r}21,741 \\
45,036 \\
81,209 \\
152,919\end{array}$ \\
\hline $396.000 \ldots$ & 80 & $--d 0=-$ & & $\begin{array}{r}50 \\
19 \\
3 \\
0\end{array}$ & $\begin{array}{r}4,030 \\
8,182 \\
16,861 \\
35,788\end{array}$ & $\begin{array}{r}101 \\
19 \\
3 \\
0\end{array}$ & $\begin{array}{r}82,832 \\
274,150 \\
931,556 \\
3,847,774\end{array}$ \\
\hline $396,000 \ldots$ & 90 & Tenfold...- & $\left\{\begin{array}{l}F_{1} \\
F_{2} \\
E_{3}\end{array}\right.$ & $\begin{array}{r}101 \\
48 \\
6 \\
3\end{array}$ & $\begin{array}{r}4,080 \\
8,694 \\
18,414 \\
43,374\end{array}$ & $\begin{array}{r}101 \\
39 \\
18 \\
12\end{array}$ & $\begin{array}{r}43,481 \\
157,060 \\
699,296 \\
4,984,656\end{array}$ \\
\hline $396,000--$ & 80 & $--10 \ldots$ & $\left\{\begin{array}{l}1 \\
E_{2} \\
E_{3}\end{array}\right.$ & $\begin{array}{l}101 \\
153 \\
116 \\
148\end{array}$ & $\begin{array}{r}8,060 \\
33,206 \\
148,238 \\
835,210\end{array}$ & $\begin{array}{r}101 \\
51 \\
34 \\
33\end{array}$ & $\begin{array}{r}165,664 \\
1,067,514 \\
8,984,906 \\
87,580,048\end{array}$ \\
\hline
\end{tabular}

Table 23,--Mmers in eacb generation in wich conditionsl lechal tralts cannot be expressed when native population of 2,000 insects MBBDDEE) was overflooded la parental, $F_{1}, F_{2}$, and $F_{3}$ generations by partially aterile insecta labbodee) vitb 2 conditionol lethal traits

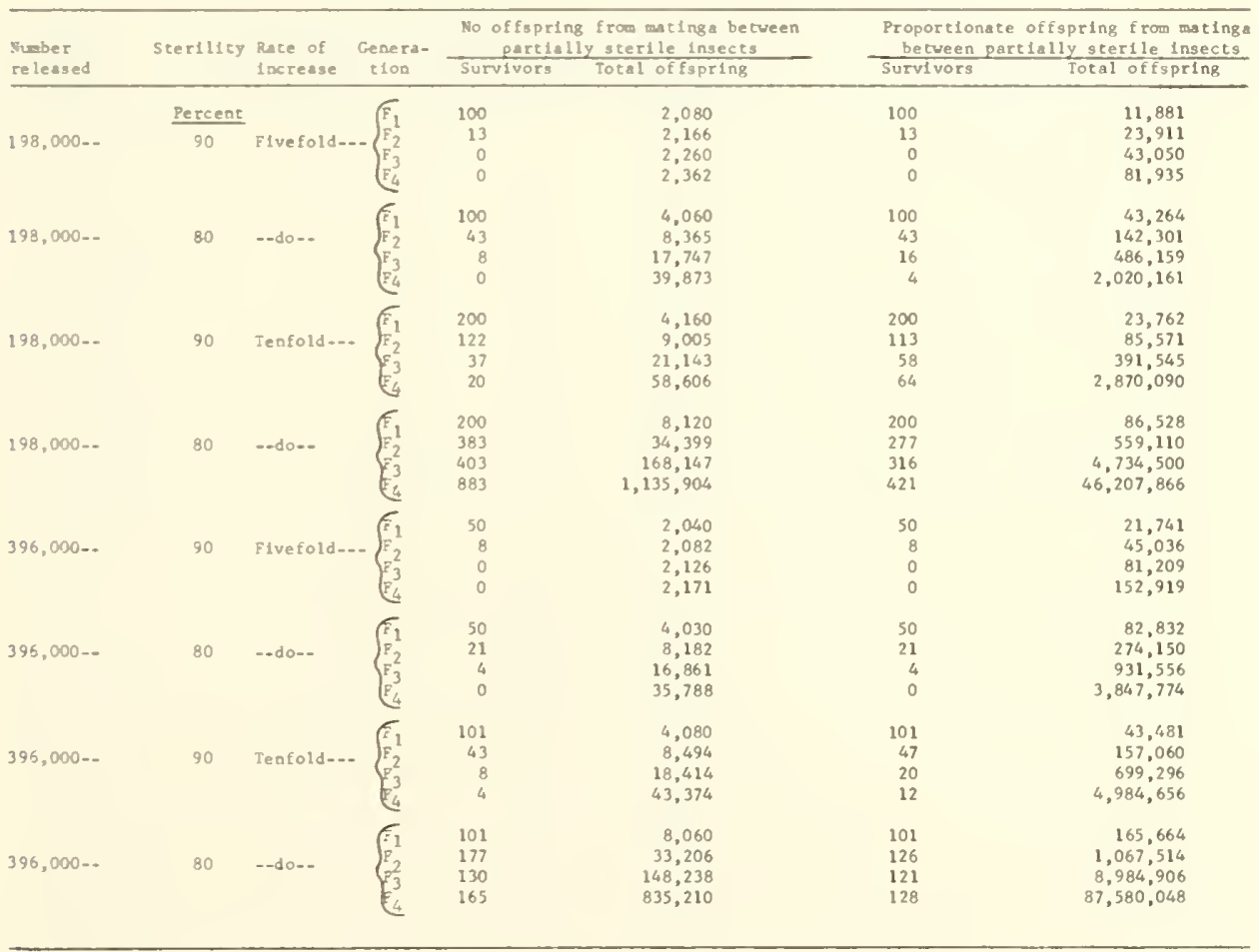


Table 24.--Numbers in each generation in which conditional lethal traits cannot be expressed when native population of 2,000 insects ( 3 autosomal genes BBDDEE plus 1 sex-linked gene aa; female is heterogametic) was overflooded in parental, $\mathrm{F}_{1}, \mathrm{~F}_{2}$, and $\mathrm{F}_{3}$ generations by partially sterile insects (AAbbddee, A-bbddee) with 2 eonditional

\begin{tabular}{|c|c|c|c|c|c|c|c|c|c|}
\hline \multirow{2}{*}{$\begin{array}{l}\text { Number } \\
\text { released }\end{array}$} & \multirow[t]{2}{*}{ Sterility } & \multirow{2}{*}{$\begin{array}{l}\text { Rate of } \\
\text { increase }\end{array}$} & \multirow{2}{*}{$\begin{array}{l}\text { Genera- } \\
\text { tion }\end{array}$} & \multicolumn{3}{|c|}{$\begin{array}{c}\text { No of fspring from matings between } \\
\text { partially sterile insects }\end{array}$} & \multicolumn{3}{|c|}{$\begin{array}{l}\text { Proportionate offspring from matings } \\
\text { between partially sterile insects }\end{array}$} \\
\hline & & & & & Females & Total & Males & Fema les & Total \\
\hline $198,000=-$ & $\frac{\text { Percent }}{90}$ & Five fold- & $\left\{\begin{array}{l}F_{1} \\
F_{3}^{2} \\
F_{4}\end{array}\right.$ & $\begin{array}{r}50 \\
7 \\
0 \\
0\end{array}$ & $\begin{array}{r}50 \\
10 \\
0 \\
0\end{array}$ & $\begin{array}{l}2,080 \\
2,166 \\
2,322 \\
2,772\end{array}$ & $\begin{array}{r}50 \\
7 \\
0 \\
0\end{array}$ & $\begin{array}{r}50 \\
10 \\
0 \\
0\end{array}$ & $\begin{array}{l}11,880 \\
23,910 \\
43,050 \\
81,934\end{array}$ \\
\hline $198,000--$ & 80 & --do-- & $\left\{\begin{array}{l}F_{1} \\
F_{2} \\
E_{4}\end{array}\right.$ & $\begin{array}{r}50 \\
22 \\
3 \\
0\end{array}$ & $\begin{array}{r}50 \\
42 \\
15 \\
3\end{array}$ & $\begin{array}{r}4,060 \\
8,364 \\
18,214 \\
45,974\end{array}$ & $\begin{array}{r}50 \\
19 \\
3 \\
3\end{array}$ & $\begin{array}{l}50 \\
32 \\
18 \\
15\end{array}$ & $\begin{array}{r}43,264 \\
142,300 \\
486,156 \\
2,020,142\end{array}$ \\
\hline $198,000=-$ & 90 & Tenfold - - & $\left\{\begin{array}{l}\mathrm{F}_{1} \\
\mathrm{~F}_{2} \\
\mathrm{~F}_{3} \\
\mathrm{~F}_{4}\end{array}\right.$ & $\begin{array}{r}100 \\
53 \\
19 \\
6\end{array}$ & $\begin{array}{r}100 \\
95 \\
49 \\
33\end{array}$ & $\begin{array}{r}4,160 \\
9,004 \\
21,610 \\
65,288\end{array}$ & $\begin{array}{r}100 \\
44 \\
27 \\
27\end{array}$ & $\begin{array}{r}100 \\
85 \\
82 \\
139\end{array}$ & $\begin{array}{r}23,762 \\
85,572 \\
391,542 \\
2,870,044\end{array}$ \\
\hline $198,000=-$ & 80 & --do-- & $\left\{\begin{array}{l}F_{1} \\
F_{2}\end{array}\right.$ & $\begin{array}{l}100 \\
168 \\
178 \\
391\end{array}$ & $\begin{array}{r}100 \\
314 \\
537 \\
1,590\end{array}$ & $\begin{array}{r}8,120 \\
34,400 \\
171,406 \\
1,206,836\end{array}$ & $\begin{array}{l}100 \\
119 \\
140 \\
187\end{array}$ & $\begin{array}{r}100 \\
228 \\
408 \\
1,093\end{array}$ & $\begin{array}{r}86,528 \\
559,110 \\
4,734,478 \\
46,207,232\end{array}$ \\
\hline $396,000=$ & 90 & Fivefold-.. & $\left\{\begin{array}{l}F_{1} \\
F_{2} \\
F_{3}\end{array}\right.$ & $\begin{array}{r}25 \\
0 \\
0 \\
0\end{array}$ & $\begin{array}{r}25 \\
6 \\
0 \\
0\end{array}$ & $\begin{array}{l}2,040 \\
2,082 \\
2,188 \\
2,582\end{array}$ & $\begin{array}{r}25 \\
0 \\
0 \\
0\end{array}$ & $\begin{array}{r}25 \\
3 \\
0 \\
0\end{array}$ & $\begin{array}{r}21,740 \\
45,036 \\
81,210 \\
152,918\end{array}$ \\
\hline $396,000--$ & 80 & --do-- & $\left\{\begin{array}{l}F_{1} \\
F_{2} \\
E_{3} \\
E_{4}\end{array}\right.$ & $\begin{array}{r}25 \\
10 \\
0 \\
0\end{array}$ & $\begin{array}{r}25 \\
19 \\
3 \\
0\end{array}$ & $\begin{array}{r}4,030 \\
8,182 \\
17,344 \\
42,124\end{array}$ & $\begin{array}{r}25 \\
6 \\
0 \\
0\end{array}$ & $\begin{array}{r}25 \\
16 \\
6 \\
3\end{array}$ & $\begin{array}{r}82,832 \\
274,150 \\
931,552 \\
3,847,732\end{array}$ \\
\hline $396,000--$ & 90 & Tenfold =- & $F_{2}^{1}$ & $\begin{array}{r}50 \\
23 \\
3 \\
0\end{array}$ & $\begin{array}{r}50 \\
42 \\
15 \\
3\end{array}$ & $\begin{array}{r}4,080 \\
8,494 \\
18,898 \\
50,022\end{array}$ & $\begin{array}{r}50 \\
19 \\
6 \\
6\end{array}$ & $\begin{array}{l}50 \\
35 \\
27 \\
39\end{array}$ & $\begin{array}{r}43,482 \\
157,060 \\
699,292 \\
4,984,602\end{array}$ \\
\hline $396,000--$ & 80 & --do-- & $\mathrm{F}_{1}$ & $\begin{array}{l}50 \\
73 \\
58 \\
75\end{array}$ & $\begin{array}{r}50 \\
146 \\
191 \\
368\end{array}$ & $\begin{array}{r}8,060 \\
33,206 \\
151,844 \\
921,802\end{array}$ & $\begin{array}{l}50 \\
54 \\
52 \\
54\end{array}$ & $\begin{array}{r}50 \\
105 \\
197 \\
379\end{array}$ & $\begin{array}{r}165,664 \\
1,067,512 \\
8,984,826 \\
87,578,912\end{array}$ \\
\hline
\end{tabular}

Table 25.--Numbers in each generation in which conditional lethal traits cannot be expressed when native population of 2,000 insects (aabbdd) was overflooded in parenta1, $F_{1}, F_{2}$, and $F_{3}$ generations by partially sterile insects (AABBDD) with 3 conditional lethal traits

\begin{tabular}{|c|c|c|c|c|c|c|c|}
\hline \multirow{2}{*}{$\begin{array}{l}\text { Number } \\
\text { released }\end{array}$} & \multirow[t]{2}{*}{ Sterility } & \multirow{2}{*}{$\begin{array}{l}\text { Rate of } \\
\text { increase }\end{array}$} & \multirow{2}{*}{$\begin{array}{l}\text { Genera- } \\
\text { tion }\end{array}$} & \multirow{2}{*}{\multicolumn{2}{|c|}{$\begin{array}{l}\text { No of fopting from matings be- } \\
\text { tween partially sterile insects } \\
\text { Survivors Total offspring }\end{array}$}} & \multicolumn{2}{|c|}{$\begin{array}{l}\text { Proportionate offspring from matings } \\
\text { between partially sterile insects }\end{array}$} \\
\hline & & & & & & Survivors & Total offspring \\
\hline $198,000=-$ & Percent & Fivefold--- & $\left\{\begin{array}{l}\mathrm{F}_{1} \\
\mathrm{~F}_{2} \\
\mathrm{~F}_{3} \\
\mathrm{~F}_{4}\end{array}\right.$ & $\begin{array}{r}100 \\
3 \\
0 \\
0\end{array}$ & $\begin{array}{l}2,080 \\
2,166 \\
2,260 \\
2,362\end{array}$ & $\begin{array}{r}100 \\
3 \\
0 \\
0\end{array}$ & $\begin{array}{l}11,881 \\
23,911 \\
43,050 \\
81,936\end{array}$ \\
\hline $198,000=-$ & 80 & -rdo=- & $\left\{\begin{array}{l}F_{1} \\
F_{2} \\
F_{3} \\
F_{4}\end{array}\right.$ & $\begin{array}{r}100 \\
9 \\
1 \\
0\end{array}$ & $\begin{array}{r}4,060 \\
8,365 \\
17,747 \\
39,873\end{array}$ & $\begin{array}{r}100 \\
7 \\
1 \\
0\end{array}$ & $\begin{array}{r}43,264 \\
142,301 \\
486,161 \\
2,020,176\end{array}$ \\
\hline $198,000--$ & 90 & Tenfold--- & $\left\{\begin{array}{l}F_{1} \\
F_{2} \\
F_{3}^{3} \\
F_{4}\end{array}\right.$ & $\begin{array}{r}200 \\
24 \\
4 \\
2\end{array}$ & $\begin{array}{r}4,160 \\
9,005 \\
21,144 \\
58,607\end{array}$ & $\begin{array}{r}200 \\
22 \\
6 \\
5\end{array}$ & $\begin{array}{r}23,762 \\
85,571 \\
391,548 \\
2,870,116\end{array}$ \\
\hline $198,000=-$ & 80 & --do-- & $\left\{\begin{array}{l}F_{1} \\
E_{2} \\
F_{3} \\
E_{4}\end{array}\right.$ & $\begin{array}{r}200 \\
69 \\
45 \\
81\end{array}$ & $\begin{array}{r}8,120 \\
34,399 \\
168,148 \\
1,135,912\end{array}$ & $\begin{array}{r}200 \\
50 \\
32 \\
33\end{array}$ & $\begin{array}{r}86,528 \\
559,111 \\
4,734,505 \\
46,207,856\end{array}$ \\
\hline $396,000 \ldots$ & 90 & Five fold --- & $\left\{\begin{array}{l}F_{1} \\
F_{2} \\
F_{3} \\
F_{4}\end{array}\right.$ & $\begin{array}{r}50 \\
1 \\
0 \\
0\end{array}$ & $\begin{array}{l}2,040 \\
2,082 \\
2,126 \\
2,171\end{array}$ & $\begin{array}{r}50 \\
1 \\
0 \\
0\end{array}$ & $\begin{array}{r}21,740 \\
45,036 \\
81,210 \\
152,920\end{array}$ \\
\hline $396,000--$ & 80 & --do-- & $\left\{\begin{array}{l}F_{1} \\
F_{2}^{2} \\
F_{3}^{3}\end{array}\right.$ & $\begin{array}{r}50 \\
4 \\
0 \\
0\end{array}$ & $\begin{array}{r}4,030 \\
8,182 \\
16,861 \\
35,788\end{array}$ & $\begin{array}{r}50 \\
3 \\
0 \\
0\end{array}$ & $\begin{array}{r}82,832 \\
274,150 \\
931,557 \\
3,847,777\end{array}$ \\
\hline $396,000=-$ & 90 & Tenfold--- & $\left\{\begin{array}{l}\mathrm{F}_{1} \\
\mathrm{~F}_{2} \\
\mathrm{~F}_{3} \\
\mathrm{~F}_{4}\end{array}\right.$ & $\begin{array}{r}101 \\
9 \\
1 \\
0\end{array}$ & $\begin{array}{r}4,081 \\
8,494 \\
18,414 \\
43,374\end{array}$ & $\begin{array}{r}101 \\
8 \\
2 \\
1\end{array}$ & $\begin{array}{r}43,482 \\
157,060 \\
699,296 \\
4,984,654\end{array}$ \\
\hline $396,000--$ & 80 & --do-- & $\left\{\begin{array}{l}F_{1} \\
F_{2} \\
F_{3} \\
F_{4}\end{array}\right.$ & $\begin{array}{r}101 \\
30 \\
13 \\
14\end{array}$ & $\begin{array}{r}8,060 \\
33,206 \\
148,237 \\
835,208\end{array}$ & $\begin{array}{r}101 \\
21 \\
12 \\
10\end{array}$ & $\begin{array}{r}165,663 \\
1,067,514 \\
8,984,898 \\
87,579,904\end{array}$ \\
\hline
\end{tabular}


Table 26.-.Nubers in each generatlon in which condicional lethal traits cannot be expressed when native population of 2,000 insects (abbDDEE) was overflooded in parental, $F_{1}, F_{2}$, and $F_{3}$ generstions by partlally sterile insects (ABBBddee) with 3 conditionsl lethal eralcs

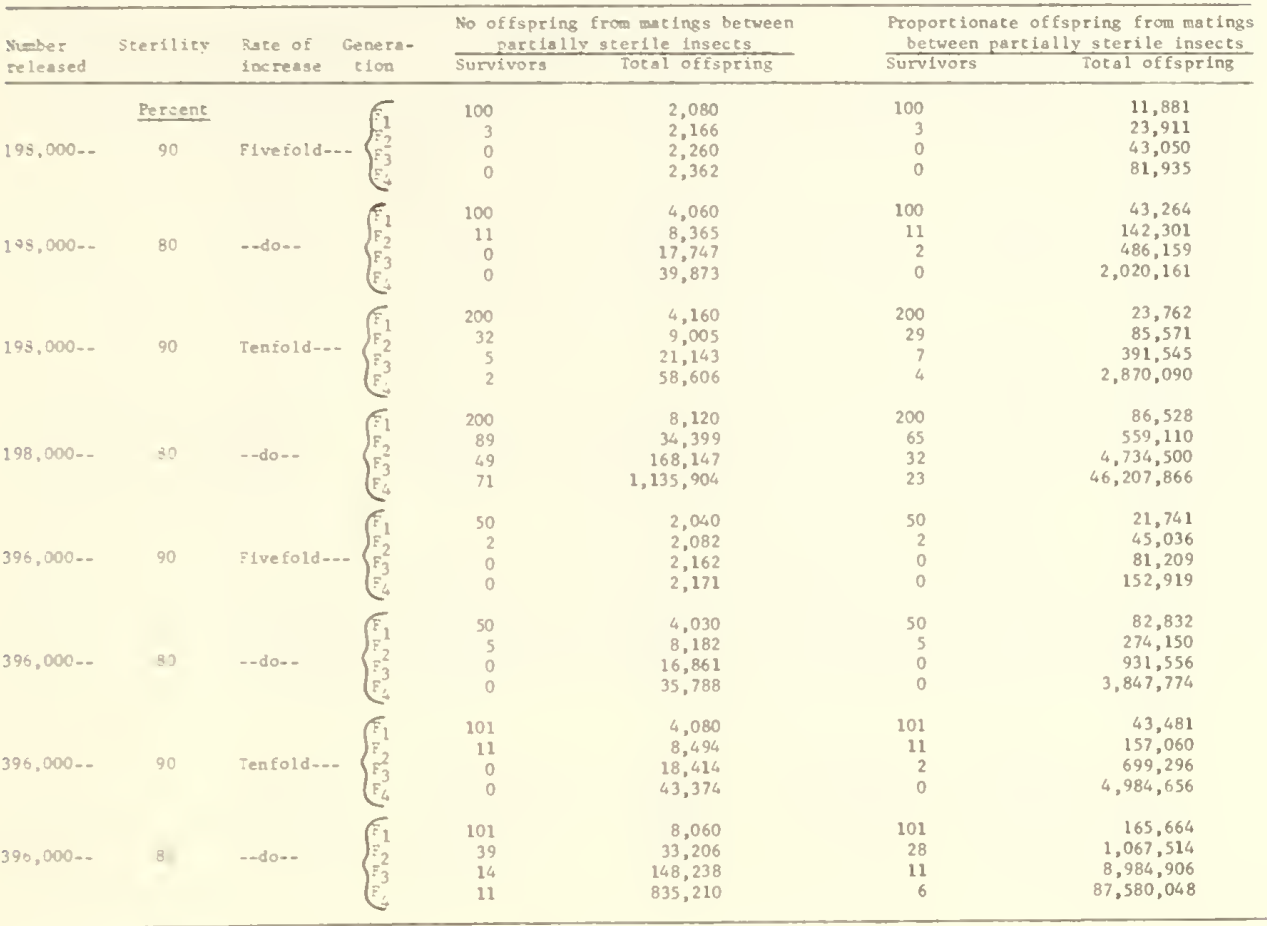

Table 27 ...-Nubers in each zeneration in which conditional lethal traits cannot be expressed when native population of 2,000 insects ( 3 autososal genes bbDDEE plus 1 sex-linked gene as; female is heterogatnetic) was overflooded in parental, $F_{1}, F_{2}$, and $F_{3}$ generations by partlally sterlle insects (AABBddee, A-BBddee) with 3 conditional lechal iraits

\begin{tabular}{|c|c|c|c|c|c|c|c|c|c|}
\hline \multirow{2}{*}{$\begin{array}{l}\text { Nuber } \\
\text { relessed }\end{array}$} & \multirow[t]{2}{*}{ scer111ty } & \multirow{2}{*}{$\begin{array}{l}\text { Rate of } \\
\text { Increase }\end{array}$} & \multirow{2}{*}{$\begin{array}{l}\text { Geners- } \\
\text { tion }\end{array}$} & \multicolumn{3}{|c|}{$\begin{array}{c}\text { No offsping from matings between } \\
\text { partially sterile insects }\end{array}$} & \multicolumn{3}{|c|}{$\begin{array}{l}\text { Proportionate of fspeling from matings } \\
\text { between partially sterile ingects }\end{array}$} \\
\hline & & & & Males & Females & Total & Males & Females & Total \\
\hline $178,000=$ & $\frac{\text { Percens }}{90}$ & Elvefold-- & $=\frac{2}{3}$ & $\begin{array}{r}50 \\
3 \\
0 \\
0\end{array}$ & $\begin{array}{r}50 \\
3 \\
0 \\
0\end{array}$ & $\begin{array}{l}2,080 \\
2,166 \\
2,322 \\
2,772\end{array}$ & $\begin{array}{r}50 \\
3 \\
0 \\
0\end{array}$ & $\begin{array}{r}\text { So } \\
3 \\
0 \\
0\end{array}$ & $\begin{array}{l}11,880 \\
23,910 \\
43,050 \\
81,934\end{array}$ \\
\hline $198,000 \ldots$ & 80 & --do-- & $E_{3}^{2}$ & $\begin{array}{r}50 \\
5 \\
0 \\
0\end{array}$ & $\begin{array}{r}50 \\
11 \\
2 \\
0\end{array}$ & $\begin{array}{r}4,060 \\
8,364 \\
18,214 \\
45,974\end{array}$ & $\begin{array}{r}50 \\
5 \\
0 \\
0\end{array}$ & $\begin{array}{r}50 \\
8 \\
2 \\
0\end{array}$ & $\begin{array}{r}43,264 \\
142,300 \\
486,156 \\
2,020,142\end{array}$ \\
\hline $198,000 \ldots$ & 90 & Tenfold-- & $F_{4}$ & $\begin{array}{r}100 \\
15 \\
2 \\
0\end{array}$ & $\begin{array}{r}100 \\
28 \\
7 \\
2\end{array}$ & $\begin{array}{r}4,160 \\
9,004 \\
21,610 \\
65,288\end{array}$ & $\begin{array}{r}100 \\
15 \\
4 \\
2\end{array}$ & $\begin{array}{r}100 \\
25 \\
9 \\
9\end{array}$ & $\begin{array}{r}23,762 \\
85,572 \\
391,542 \\
2,870,044\end{array}$ \\
\hline $198,000=-$ & 80 & - -does & $\varepsilon^{2}$ & $\begin{array}{r}100 \\
46 \\
26 \\
35\end{array}$ & $\begin{array}{r}100 \\
82 \\
69 \\
138\end{array}$ & $\begin{array}{r}8,120 \\
34,400 \\
171,405 \\
1,206,836\end{array}$ & $\begin{array}{r}100 \\
32 \\
16 \\
11\end{array}$ & $\begin{array}{r}100 \\
59 \\
52 \\
61\end{array}$ & $\begin{array}{r}86,528 \\
559,110 \\
4,734,478 \\
46,207,232\end{array}$ \\
\hline $395,000=$ & 90 & Five fold- & & $\begin{array}{r}25 \\
0 \\
0 \\
0\end{array}$ & $\begin{array}{r}25 \\
2 \\
0 \\
0\end{array}$ & $\begin{array}{l}2,040 \\
2,082 \\
2,188 \\
2,582\end{array}$ & $\begin{array}{r}25 \\
0 \\
0 \\
0\end{array}$ & $\begin{array}{r}25 \\
0 \\
0 \\
0\end{array}$ & $\begin{array}{r}21,740 \\
45,036 \\
81,210 \\
152,918\end{array}$ \\
\hline $396,000=$ & 80 & $=-d 0 \ldots$ & 6 & $\begin{array}{r}25 \\
3 \\
0 \\
0\end{array}$ & $\begin{array}{r}25 \\
5 \\
0 \\
0\end{array}$ & $\begin{array}{r}4,030 \\
8,182 \\
17,344 \\
42,124\end{array}$ & $\begin{array}{r}25 \\
2 \\
0 \\
0\end{array}$ & $\begin{array}{r}25 \\
3 \\
0 \\
0\end{array}$ & $\begin{array}{r}82,832 \\
274,150 \\
931,552 \\
3,847,732\end{array}$ \\
\hline $396,000=$ & 90 & Tenfold-- & $\left\{\begin{array}{l}F_{3}^{2} \\
E_{4}^{3}\end{array}\right.$ & $\begin{array}{r}50 \\
8 \\
0 \\
0\end{array}$ & $\begin{array}{r}50 \\
11 \\
2 \\
0\end{array}$ & $\begin{array}{r}4,080 \\
8,494 \\
18,808 \\
50,022\end{array}$ & $\begin{array}{r}50 \\
5 \\
0 \\
0\end{array}$ & $\begin{array}{r}50 \\
10 \\
2 \\
2\end{array}$ & $\begin{array}{r}43,482 \\
157,060 \\
699,292 \\
4,984,602\end{array}$ \\
\hline $395,000=$ & 80 & --do.. & $\left\{\begin{array}{l}F_{1} \\
F_{2} \\
F_{3} \\
F_{1}\end{array}\right.$ & $\begin{array}{r}50 \\
18 \\
7 \\
6\end{array}$ & $\begin{array}{l}50 \\
36 \\
20 \\
26\end{array}$ & $\begin{array}{r}8,060 \\
33,206 \\
151,844 \\
921,802\end{array}$ & $\begin{array}{r}50 \\
13 \\
5 \\
2\end{array}$ & $\begin{array}{l}50 \\
26 \\
20 \\
19\end{array}$ & $\begin{array}{r}165,664 \\
1,067,512 \\
8,984,826 \\
87,578,912\end{array}$ \\
\hline
\end{tabular}


Table 28.--Complete suppression of native population $\left(2,000\right.$ insects, $1: 1$ sex ratio) by overflooding parental, $F_{1}$, $F_{2}$, and $F_{3}$ generations with partially sterile release strain bearing conditional lethal traits. Complete suppression is assumed when number of insects with genotypes for survival is reduced to 30 or less

\begin{tabular}{|c|c|c|c|c|c|c|c|c|c|}
\hline \multicolumn{4}{|c|}{$\begin{array}{c}\text { Inheritance of conditional } \\
\text { lethal traits in } \\
\text { release strain }\end{array}$} & \multicolumn{3}{|c|}{$\begin{array}{l}\text { No offspring from partially } \\
\text { sterile insect matings: when } \\
\text { lethal traits expressed during-- }\end{array}$} & \multicolumn{3}{|c|}{$\begin{array}{c}\text { Proportionate offspring from partially } \\
\text { sterile insect matings when lethal } \\
\text { traits expressed during-- }\end{array}$} \\
\hline & Trait 1 & Trait 2 & Trait 3 & $\mathrm{~F}_{2}$ & $\mathrm{~F}_{3}$ & $\mathrm{~F}_{4}$ & $\mathrm{~F}_{2}$ & $F_{3}$ & $\mathrm{~F}_{4}$ \\
\hline & $\begin{array}{l}\text { dom.auto. } \\
\text { auto. }(4) \\
\text { auto. }(3,4) \\
\text { auto. }(5,6) \\
\text { auto. }(4-6)\end{array}$ & $\begin{array}{l}-- \\
-- \\
-- \\
--\end{array}$ & $\begin{array}{l}-- \\
=- \\
-- \\
--\end{array}$ & $\begin{array}{l}1,5 \\
1,2,5,6,7 \\
5 \\
1,5,6 \\
5\end{array}$ & $\begin{array}{l}1,5 \\
1,2,5,6,7 \\
1,5 \\
1,2,5,6,7 \\
1,5\end{array}$ & $\begin{array}{l}1,5 \\
1,2,3,5,6,7 \\
1,5 \\
1,2,3,5,6,7 \\
1,5,6\end{array}$ & $\begin{array}{l}1,5 \\
1,2,5,6,7 \\
5 \\
1,5,6 \\
5\end{array}$ & $\begin{array}{l}1,5 \\
1,2,5,6,7 \\
1,5 \\
1,2,5,6,7 \\
1,5\end{array}$ & $\begin{array}{l}1,5 \\
1,2,5,6 \\
1,5 \\
1,2,5,6 \\
1,5\end{array}$ \\
\hline $\begin{array}{l}3 \\
1 \\
1\end{array}$ & $\begin{array}{l}\text { auto. }(6-8) \\
\text { auto. }+1 \text { s. } 1 .(6-9) \\
\text { dom. auto. } \\
\text { dom. auto. }\end{array}$ & $\begin{array}{l}\text {-- } \\
1 \text { dom, auto. } \\
2 \text { auto. }(3,4)\end{array}$ & $\begin{array}{l}-- \\
-- \\
--\end{array}$ & $\begin{array}{l}1,5 \\
-- \\
1,2,5,6,7 \\
1,5,6\end{array}$ & $\begin{array}{c}1,2,3,5,6, \\
7,8 \\
1,2,5,6,7 \\
1,5 \\
1,2,5,6,7 \\
1,2,5,6,7\end{array}$ & $\begin{array}{c}1,2,3,5,6, \\
7,8 \\
1,2,3,5,6,7 \\
1,5 \\
1,2,3,5,6,7 \\
1,2,3,5,6,7\end{array}$ & $\begin{array}{l}1,2,5,6,7 \\
1,5,6 \\
-- \\
1,2,5,6,7 \\
1,5,6\end{array}$ & $\begin{array}{l}1,2,3,5,6, \\
7,8 \\
1,2,5,6,7 \\
5 \\
1,2,5,6 \\
1,2,5,6,7\end{array}$ & $\begin{array}{l}1,2,5,6,7 \\
1,5 \\
1,2,5,6 \\
1,2,5,6,7\end{array}$ \\
\hline $\begin{array}{l}1 \\
1 \\
1\end{array}$ & $\begin{array}{l}\text { dom.auto, } \\
\text { auto. }(3,4) \\
\text { dona. s.i. }\end{array}$ & $\begin{array}{l}3 \text { auto. }(4-6) \\
2 \text { auto. }(3,4) \\
3 \text { auto. }(4-6)\end{array}$ & $\begin{array}{l}-- \\
--\end{array}$ & $\begin{array}{l}1,5,6 \\
1,5,6 \\
1,5,6\end{array}$ & $\begin{array}{l}1,2,5,6,7 \\
1,2,5,6,7 \\
1,2,5,6,7\end{array}$ & $\begin{array}{l}1,2,3,5,6,7 \\
1,2,3,5,6,7 \\
1,2,5,6,7\end{array}$ & $\begin{array}{l}1,5,6 \\
1,5,6 \\
1,5,6\end{array}$ & $\begin{array}{c}1,2,5,6,7 \\
1,2,5,6,7 \\
1,2,5,6,7 \\
7(?) \underline{1}\end{array}$ & $\begin{array}{l}1,2,5,6,7 \\
1,2,5,6,7 \\
1,2,5,6,7(?)\end{array}$ \\
\hline 1 & don. auto. & 1 dom auto. & $\begin{array}{l}1 \text { dom.auto. } \\
2 \text { auto. }(3,4)\end{array}$ & $\begin{array}{c}1,2,3,5,6 \\
7,8 \\
1,2,5,6,7 \\
1,2,5,6,7\end{array}$ & $\begin{array}{c}1,2,3,5,6, \\
7,8 \\
1,2,3,5,6 \\
7,8 \\
1,2,3,5,6 \\
7,8\end{array}$ & $\begin{array}{c}1,2,3,5,6,7 \\
8 \\
1,2,3,5,6,7 \\
8 \\
1,2,3,5,6,7, \\
8(?)\end{array}$ & $\begin{array}{c}1,2,3,5,6 \\
7,8 \\
1,2,3,5,6 \\
7,8 \\
1,2,5,6,7\end{array}$ & $\begin{array}{c}1,2,3,5,6, \\
7,8 \\
1,2,3,5,6, \\
7,8 \\
1,2,3,5,6, \\
7,8\end{array}$ & $\begin{array}{l}1,2,3,4,5,6,7 \\
8 \\
1,2,3,5,6,7,8\end{array}$ \\
\hline
\end{tabular}

1/ $(?)=$ case with slightly more than 30 survivors or with pronounced sex ratio distortion or with both. 
Table 29.--Relative frequencies of genotypes when native population (aa) is overflooded once in parental generation by $\underline{X}$ release insects (AA) for every native insect

\begin{tabular}{|c|c|c|c|c|}
\hline Genotype & $F_{1}$ & $\mathrm{~F}_{2}$ & $\mathrm{~F}_{3}$ & $F_{4}$ \\
\hline & & $\underline{X}$ & $=1$ & \\
\hline a a... & 0.2500000 & 0.2500000 & 0.2500000 & 0.2500000 \\
\hline Aa-.- & .5000000 & .5000000 & .5000000 & .5000000 \\
\hline \multirow[t]{2}{*}{$A A-=$} & .2500000 & .2500000 & .2500000 & .2500000 \\
\hline & & $\underline{x}$ & $=2$ & \\
\hline a a-.. & .1111111 & .1111111 & .1111111 & .1111111 \\
\hline $\mathrm{Aa}---$ & .4444444 & .4444444 & .4444444 & .4444444 \\
\hline \multirow[t]{2}{*}{ AA- - } & .4444444 & $.44441+44$ & .4444444 & .4444444 \\
\hline & & $\underline{x}=$ & $=3$ & \\
\hline aa--- & .0625000 & .0625000 & .0625000 & .0625000 \\
\hline Aa--- & .3750000 & .3750000 & .3750000 & .3750000 \\
\hline \multirow[t]{2}{*}{$A A-\ldots$} & .5625000 & .5625000 & .5625000 & .5625000 \\
\hline & & $\underline{x}$ & $=4$ & \\
\hline a a--- & .0400000 & .0400000 & .0400000 & .0400000 \\
\hline $\mathrm{Aa}---$ & .3200000 & .3200000 & .3200000 & .3200000 \\
\hline \multirow[t]{2}{*}{$A A-=-$} & .6400000 & .6400000 & .6400000 & .6400000 \\
\hline & & $\underline{x}$ & $=5$ & \\
\hline a a --- & .0277777 & .0277777 & .0277777 & .0277777 \\
\hline Aa_-- & .2777777 & .2777777 & .2777777 & .2777777 \\
\hline \multirow[t]{2}{*}{ AA.--} & .6944444 & .6944444 & .6944444 & .6944444 \\
\hline & & $\underline{X}$ & $=10$ & \\
\hline$a a---$ & .0082645 & .0082645 & .0082645 & .0082645 \\
\hline $\mathrm{Aa}---$ & .1652892 & .1652892 & .1652892 & .1652892 \\
\hline \multirow[t]{2}{*}{ AA ---} & .8264462 & .8264462 & .8264462 & .8264462 \\
\hline & & $\underline{\mathrm{X}}$ & $=50$ & \\
\hline a a $\ldots$ & .0003845 & .0003845 & .0003845 & .0003845 \\
\hline Aa_-- & .0384467 & .0384467 & .0384467 & .0384467 \\
\hline \multirow[t]{2}{*}{$A A_{-} \ldots$} & .9611687 & .9611687 & .9611687 & .9611687 \\
\hline & & $\underline{x}$ & $=100$ & \\
\hline a a --- & .0000980 & .0000980 & .0000980 & .0000980 \\
\hline$A a_{-.}$ & .0196059 & .0196059 & .0196059 & .0196059 \\
\hline \multirow[t]{2}{*}{$A A_{-.}$} & .9802959 & .9802959 & .9802959 & .9802959 \\
\hline & & $\underline{x}$ & $=200$ & \\
\hline aa--- & .0000248 & .0000248 & .0000248 & .0000248 \\
\hline Aa -.- & .0099007 & .0099007 & .0099007 & .0099007 \\
\hline \multirow[t]{2}{*}{$A A_{---}$} & .9900745 & .9900745 & .9900745 & .9900745 \\
\hline & & $\underline{x}$ & $=1,000$ & \\
\hline a --- & .0000010 & .0000010 & .0000010 & .0000010 \\
\hline$A a_{-\ldots}$ & .0019960 & .0019960 & .0019960 & .0019960 \\
\hline$A A_{-.}$ & .9980029 & .9980029 & .9980029 & .9980029 \\
\hline
\end{tabular}


Table 30.--Relative frequencies of genotypes when native population (AABB) is overflooded once in parental generation bý $x$ release insects (aabb) for every native insect

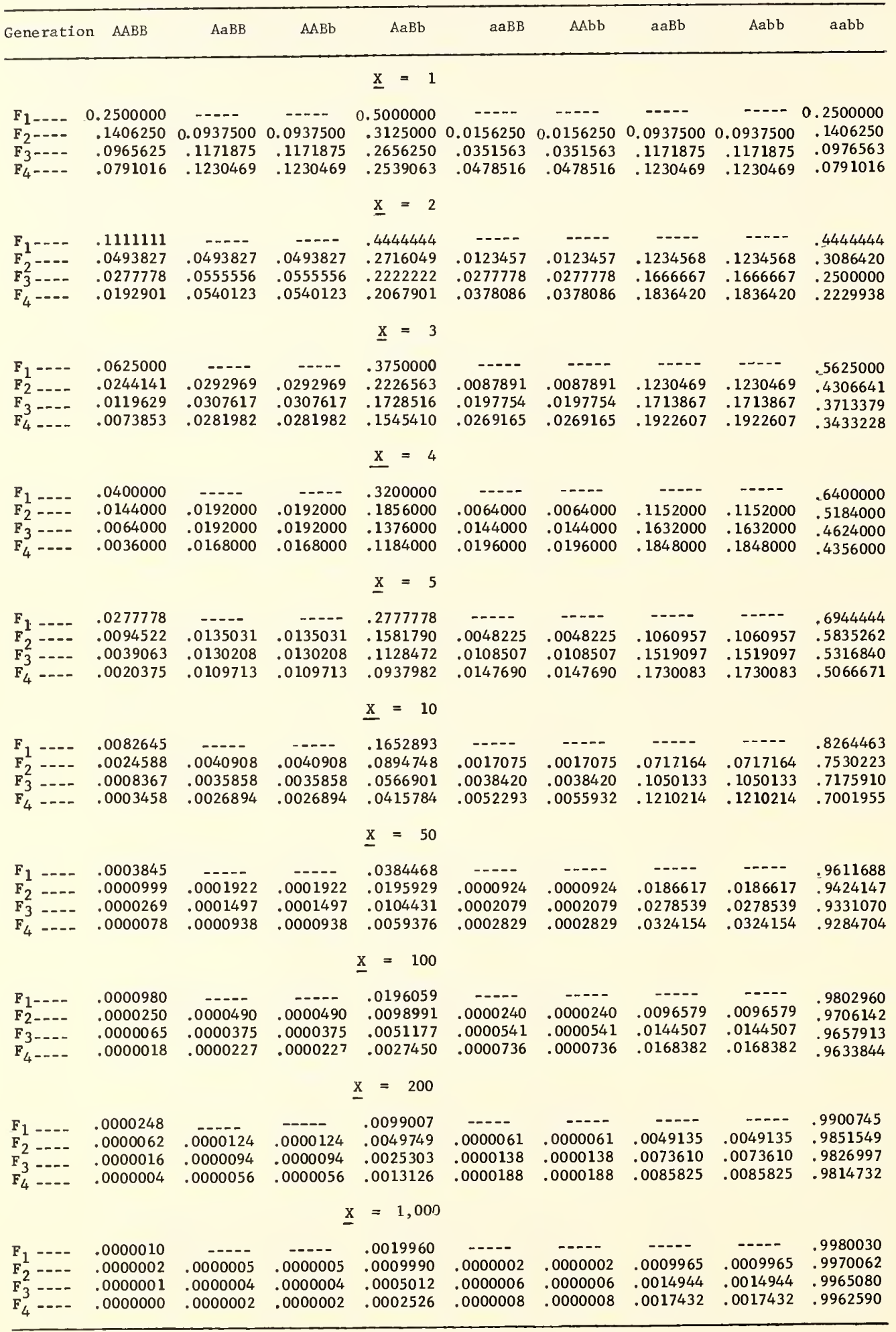




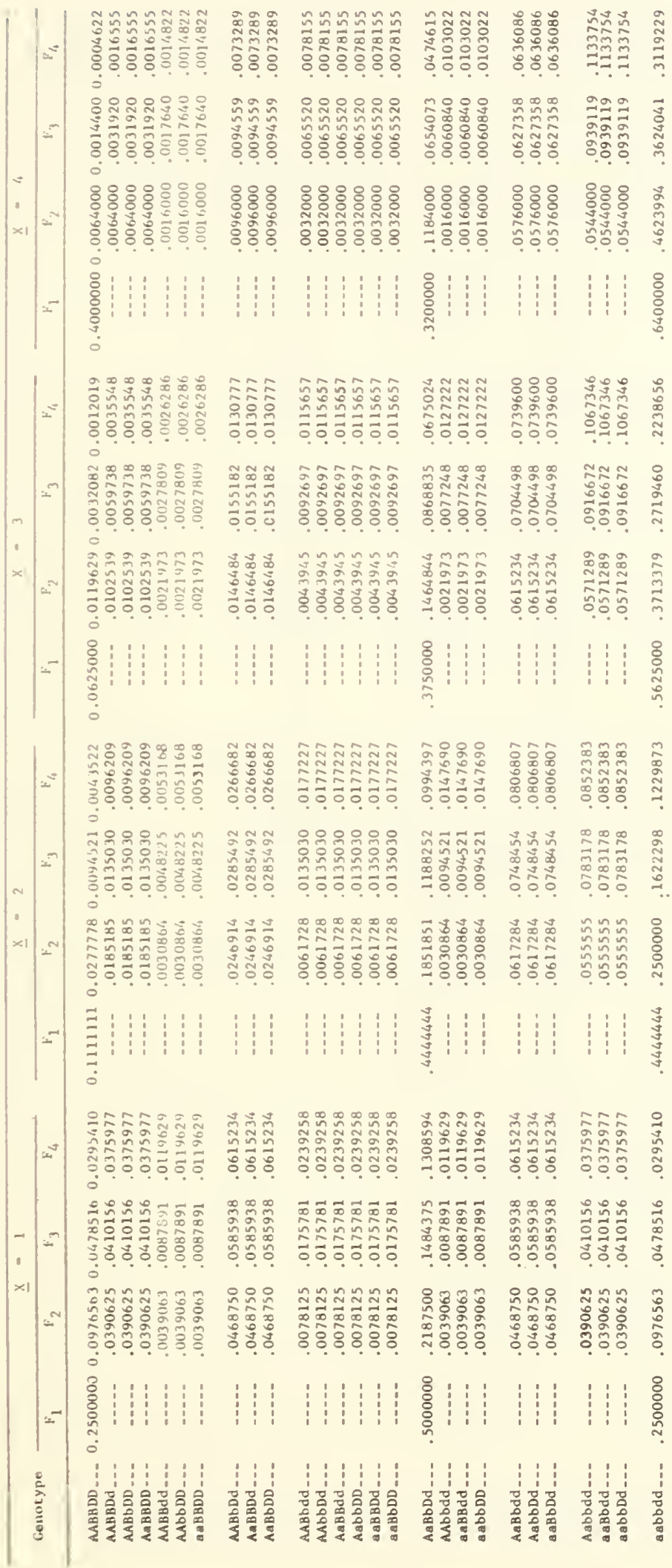




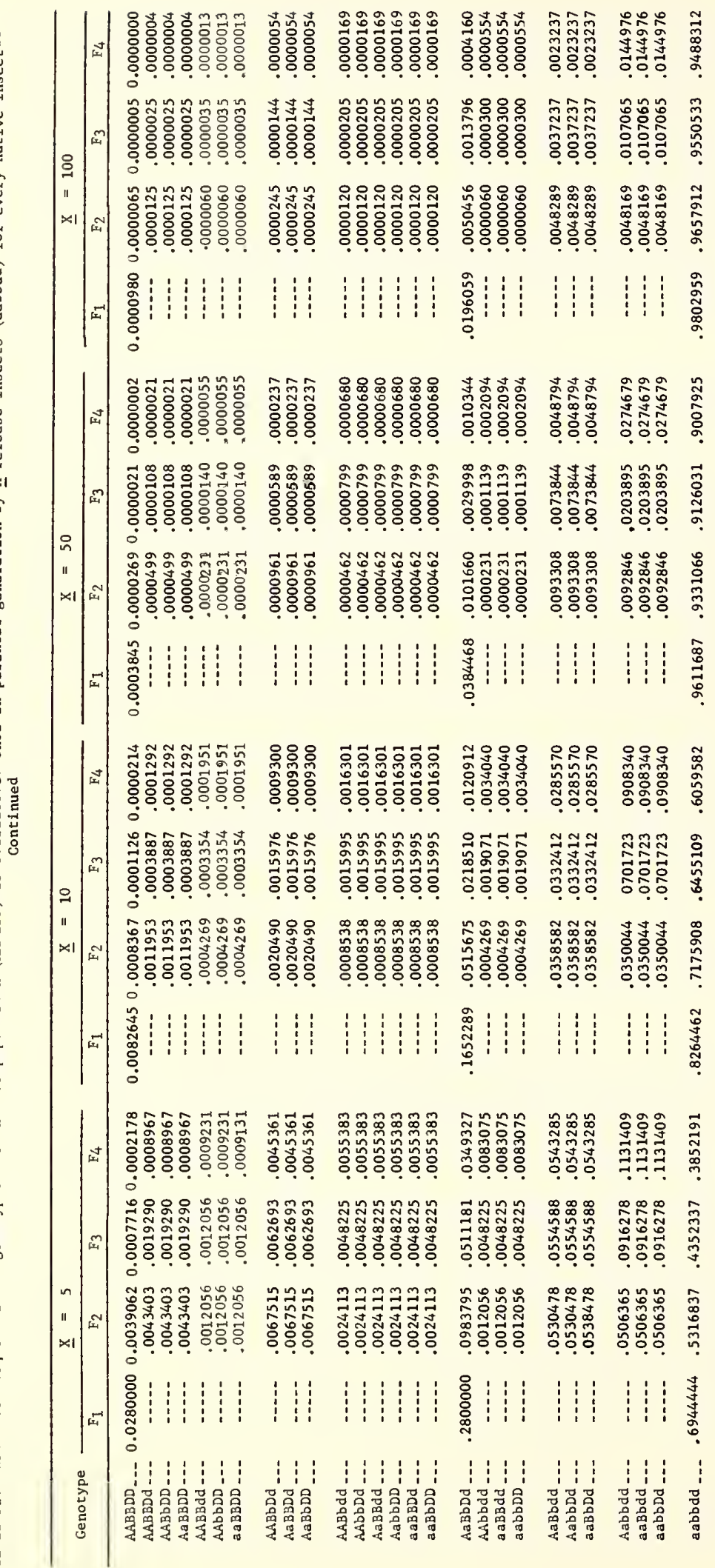


Table 31.--Relative frequencies of genotypes when native population (AABBDD) is overflooded once in parental generation by $\underline{X}$ release insects (aabbdd) for every native insect-Continued

\begin{tabular}{|c|c|c|c|c|c|c|c|c|}
\hline \multirow{2}{*}{ Genotype } & \multicolumn{4}{|c|}{$\underline{x}=200$} & \multicolumn{4}{|c|}{$\underline{x}=1,000$} \\
\hline & $F_{1}$ & $F_{2}$ & $\mathrm{~F}_{3}$ & $\mathrm{~F}_{4}$ & $F_{1}$ & $\mathrm{~F}_{2}$ & $\mathrm{~F}_{3}$ & $F_{4}$ \\
\hline AAESD _.. & 0,0000248 & 0.0000016 & 0.0000001 & 0.0000001 & 0.0000010 & 0.0000001 & 0.0000000 & 0.0000000 \\
\hline Ais $500^{\circ}$ & -- & .0000031 & .0000006 & .0000010 & $\ldots .-$ & .0000001 & .0000000 & .0000000 \\
\hline AS3DD _.. & $-\ldots$ & .0000031 & .0000006 & .0000010 & $\ldots$ & .0000001 & .0000000 & .0000000 \\
\hline ม่aริธD ... & $-\ldots$ & .0000031 & .0000006 & .0000010 & ---- & .0000001 & .0000000 & .0000000 \\
\hline ASSEd -. - & ---- & .0000015 & .0000009 & .0000003 & $\ldots-$ & .0000001 & .0000000 & .0000000 \\
\hline AMSOD _. & $\ldots$ & .0000015 & .0000009 & .0000003 & -.... & .0000001 & .0000000 & .0000000 \\
\hline a a 5300 & $-\ldots$ & .0000015 & .0000009 & .0000003 & $\cdots+$ & .0000001 & .0000000 & .0000000 \\
\hline Assb & 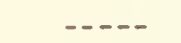 & .0000062 & .0000036 & .0000013 & -... & .0000002 & .0000001 & .0000000 \\
\hline Aa $332 \mathrm{C} . .$. & $\ldots$ & .0000062 & .0000036 & .0000013 & $\ldots$ & .0000002 & .0000001 & .0000000 \\
\hline 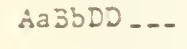 & $\ldots$ & .0000062 & .0000036 & .0000013 & $\ldots--$ & .0000002 & .0000001 & .0000000 \\
\hline Aisbdd ... & $\ldots$ & .0000031 & .0000052 & .0000042 & ---- & .0000001 & .0000002 & .0000002 \\
\hline AADBDC _.. & ---- & .0000031 & .0000052 & .00000 & -.-.- & .0000001 & .0000002 & .0000002 \\
\hline saBs̄d _. - & ---- & .0000031 & .0000052 & .0000042 & ---- & .0000001 & .0000002 & .0000002 \\
\hline AabbDD ... & $\ldots-$ & .0000031 & .0000052 & .0000042 & $-\ldots$ & .0000001 & .0000002 & .0000002 \\
\hline a aBBDC ... & --- & .0000031 & .0000052 & .0000042 & ---- & .0000001 & .0000002 & .0000002 \\
\hline$a a B b D D \ldots$ & $-\ldots$ & .0000031 & .0000052 & .0000042 &.--- & .0000001 & .0000002 & .0000002 \\
\hline$A a B b D d \ldots$ & .0099007 & .0025119 & .0006580 & .000182 & .0019960 & .00050 & 01263 & .0000323 \\
\hline AAbbCd ... & -..-- & .0000015 & .0000077 & .0000142 & - . - & .0000001 & .0000003 & .0000006 \\
\hline a aBBdd $\ldots$ & $\ldots-$. & .0000015 & .0000077 & .0000142 & $\ldots$ & .0000001 & .0000003 & .0000006 \\
\hline a $a b b D D \ldots$ & $\ldots-$ & .0000015 & .0000077 & .0000142 & ---- & .0000001 & .0000003 & .0000006 \\
\hline AaBbdd ... & $-\cdots$ & .0024567 & .0018688 & .001129 & $\ldots$. & .0004983 & .0003748 & .0002202 \\
\hline AabbDd ... & ..... & .0024567 & .0018688 & .0011291 & $-\ldots$ & .0004983 & .0003748 & .0002202 \\
\hline a aBbDd ... & $-\ldots$ & .0024567 & .0018688 & .0011291 & ---- & .0004983 & .0003748 & .0002202 \\
\hline Áçbdd... & -.... & .0024537 & .0058471 & .0074492 & & .0004981 & .0011194 & .0015229 \\
\hline a aBbCd ... & --- & .0024537 & .0058471 & .0074492 & $\ldots-\ldots$ & .0004981 & .0011194 & .0015229 \\
\hline$a a b b D d \ldots$ & -... & .0024537 & .0058471 & .0074492 & ---- & .0004981 & .0011194 & .0015229 \\
\hline a abbc C ... & .9900745 & .9826995 & .9772047 & .9740093 & .9980030 & .9965084 & .9953883 & .9947354 \\
\hline
\end{tabular}


Table 32.--Relative frequencies of genotypes when native population (AABBDDEE) is overflooded once in parental generation by $\underline{x}$ release insects (aabddee) for every native insect

\begin{tabular}{|c|c|c|c|c|c|c|c|c|c|c|c|c|}
\hline \multirow{2}{*}{ Genotype } & & $\underline{x}$ & $=1$ & & & $\underline{x}$ & 2 & & & $\underline{x}$ & 3 & \\
\hline & $F_{1}$ & $\mathrm{~F}_{2}$ & $\mathrm{~F}_{3}$ & $\mathrm{~F}_{4}$ & $\mathrm{~F}_{1}$ & $\mathrm{~F}_{2}$ & $E_{3}$ & $\mathrm{~F}_{4}$ & $F_{1}$ & $\mathrm{~F}_{2}$ & $\mathrm{~F}_{3}$ & $\mathrm{~F}_{4}$ \\
\hline AABBDDEE_ 0.2 & 500000 & 0.0791016 & 0.0275612 & 0.0125576 & 0.1111111 & 0.0192901 & 0.0040523 & 0.0011956 & 0.0625000 & 0.0073853 & 0.0011269 & 0.0002485 \\
\hline AABBDDEe--- & ---- & .0175781 & .0175095 & .0134057 & -...- & .0077160 & .0042733 & .0021711 & --- & .0040283 & .0015490 & .0009960 \\
\hline AABBDdEE--- & ---- & .0175781 & .0175095 & .0134057 & $\cdots$ & .0077160 & .0042733 & .0021711 & $\ldots$ & .0040283 & .0015490 & .0005960 \\
\hline AABbDDEE--- & 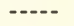 & .0175781 & .0175095 & .0134057 & -... & .0077160 & .0042733 & .0021711 & -.... & .0040283 & .0015490 & .0005960 \\
\hline AaBBDDEE--- & ---- & .0175781 & .0175095 & .0134057 & $\cdots$ & .0077160 & .0042733 & .0021711 & --- & .0040283 & .0015490 & .0005960 \\
\hline AABBDDee $=-$ & ---- & .0009766 & .0027809 & .0035778 & --- & .0007716 & .0011266 & .0009856 & $\cdots$ & .0005493 & .0005323 & .0003574 \\
\hline AABBdddEE--- & $-\cdots$ & .0009766 & .0027809 & .0035778 & $\cdots$ & .0007716 & .0011266 & .0009856 & --- & .00005493 & .0005323 & .0003574 \\
\hline AAb bDDEE - - & $-\cdots-$ & .0009766 & .0027809 & .0035778 & $\cdots$ & .0007716 & .0011266 & .0009856 & $--\cdot-$ & .00005493 & .0005323 & .0003574 \\
\hline aABBDDEE--- & ---- & .0009766 & .0027809 & .0035778 & $\ldots+$ & .0007716 & .0011266 & .0009856 & $-\cdots$ & .0005493 & .0005323 & .0003574 \\
\hline AAB BDdEe $=-$ &.--- & .0195313 & .0191803 & .0182631 & ---- & .0092593 & .0068212 & .0048427 & $\cdots$ & .0051270 & .0030561 & .0017351 \\
\hline ABbDDEe =-- & $-\cdots$ & .0195313 & .0191803 & .0182631 & $-\cdots$ & .0092593 & .0068212 & .0048 & $-\cdots$ & .0051270 & .0030561 & .0017351 \\
\hline AABbDdEE - -- & ---- & .0195313 & .0191803 & .0182631 & 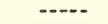 & .0092593 & .0068212 & .0048 & $\ldots$ & .0051270 & .0030561 & .0017351 \\
\hline $\mathrm{AaBBDDE}$-..- & $\ldots+$ & .0195313 & .0191803 & .0182631 & $\cdots-$ & .0092593 & .0068212 & .0048427 & $-\ldots$ & .0051270 & .0030561 & .0017351 \\
\hline AaBBDdEE $=-$ & $-\ldots$ & .0195313 & .0191803 & .0182631 & ---- & .0092593 & .0068212 & .0048427 & ---- & .0051270 & .0030561 & .0017351 \\
\hline AaBbDDEE --- & ---- & .0195313 & .0191803 & .0182631 & $-\cdots$ & .0092593 & .0068212 & .0048427 & $-\cdots$ & .0051270 & .0030561 & .0017351 \\
\hline BBDdee -- - & $\cdots$ & .0019531 & .0043259 & .0059289 & $-\ldots$ & .0015432 & .0024086 & .0026071 & & .0010986 & .0013687 & .0012237 \\
\hline ABBddEe -.- & $-\cdots$ & .0019531 & .0043259 & .0059 & ---- & .0015432 & .0024086 & & -.-- & .0010986 & & \\
\hline BbDDee --- & $\ldots$ & .0019531 & .0043259 & .0059289 & ---- & .0015432 & .00 & & 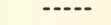 & .0010986 & & \\
\hline ABbddEE --- & $\cdots$ & .0019531 & .0043259 & .0059289 & --- & .0015432 & 24086 & & & .0010986 & & \\
\hline AAbbDDEe--- & $-\cdots$ & .0019531 & .0043259 & .0059289 & -... & .0015432 & .0024086 & .002 & -...- & .0010986 & .00 & 2237 \\
\hline AAbbDdEE --- & $\cdots-$ & .0019531 & .0043259 & .005 & -+--- & .0015432 & .00 & & $\cdots$ & .0010986 & & 12237 \\
\hline AaBBDDee - - & $\cdots$ & .0019531 & .0043259 & .0059289 & & .0015432 & .0024086 & .0026 & & .0010986 & 3687 & .0012227 \\
\hline AaBBंddEE -- & $\cdots$ & .0019531 & .0043259 & .0059 & & .0015432 & .0024 & .0 & & .0010986 & .001 & 2227 \\
\hline AabbDDEE _-- & --- & .0019531 & .0043259 & .0059289 & & .0015432 & & & $\cdots$ & .0010986 & & \\
\hline aaBBDDEe _-- & -...- & .0019531 & .0043259 & .0059289 & & .0015432 & 24086 & .00 & $-\cdots$ & .0010986 & 687 & .0012237 \\
\hline aaBBDdEE & --.-- & .0019531 & .0043259 & .0059289 & & .0015432 & .0024086 & .0026071 & ---- & .0010986 & .0013687 & .0012237 \\
\hline a aBbDDEE & $-\ldots$ & .0019531 & .0043259 & .0059289 & $\cdots$ & .0015432 & .0024086 & .0026071 & $-\cdots$ & .0010986 & .0013687 & .0012237 \\
\hline AABbDdEe --- & & .0234375 & .0304871 & .031 & - & 3457 & & & - & & & \\
\hline AaBBDdEe -.-- & --- & .0234375 & .0304871 & .031192 & & .0123457 & .0150356 & & -.. & .0073242 & & 2067 \\
\hline AaBbDDEe ... & -... & .0234375 & .0304871 & .0311923 & --..- & .0123457 & .0150356 & .0133542 & -... & .0073242 & .0080155 & .0062067 \\
\hline AaBbDdRE _.-- &.--- & .0234375 & .0304871 & .0311923 & ---- & .0123457 & .0150356 & .0133542 & $\ldots$ & .0073242 & .0080155 & .0062067 \\
\hline$e e=$. & & 9766 & .00 & & & .000 & & & & & & \\
\hline AAbbDDee --- & & .0009766 & .0016823 & .002 & & .0007716 & 12873 & & & .0005493 & 99 & 10474 \\
\hline AAbbddEE _.-- & $\ldots$ & .0009766 & .0016823 & .0024562 & & .0007716 & .0012873 & .0017241 & ---- & .0005493 & .0008799 & .0010474 \\
\hline aABBDDee ..- & -- & .0009766 & .0016823 & .0024562 & -.... & .0007716 & .0012873 & 7241 & --.- & .0005493 & .0008799 & .0010474 \\
\hline aaBBddEE -.- &.-- & .0009766 & .0016823 & .0024562 & -- & .0007716 & .0012873 & 17241 & & .0005493 & .0008799 & .0010474 \\
\hline a abbDDEE _.-- &.-- & .0009766 & .0016823 & .0024562 & 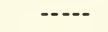 & .0007716 & .0012873 & .0017241 & $-\cdots-$ & .0005493 & .0008799 & .0010474 \\
\hline$e e_{\ldots+}$ & & .0039063 & .0089264 & .012 & & .003 & & & $\cdots$ & .0021973 & .00 & \\
\hline e... & $\ldots$ & .0039063 & .0089264 & & & .003 & 6926 & & & .0021973 & & .0 \\
\hline abbDdEe --- & $\cdots$ & .0039063 & .0089264 & .0120680 & $\cdots$ & .0030864 & 66926 & & $\cdots$ & .0021973 & .0044466 & \\
\hline AaBBDdee --- & ---- & .0039063 & .0089264 & .0120680 & -... & .0030864 & .0066926 & .0084717 & $-\cdots-$ & .0021973 & .0044466 & .0051360 \\
\hline AaBBddEe ... & ----- & .0039063 & .0089264 & .0120680 & & .0030864 & .0066926 & .00 & & .0021973 & .0044466 & 51360 \\
\hline AaBbDDee --- & -..- & .0039063 & .0089264 & .012 & & .0030864 & .0066926 & .0084717 & --- & .0021973 & .0044466 & \\
\hline AaBbddEE _.- & ---- & .0039063 & .0089264 & .0120680 & --- & .0030864 & .0066926 & .0084717 & & .0021973 & .0044466 & .0051360 \\
\hline AabbDDEe ... &..-- & .0039063 & .0089264 & .0120680 & & .0030864 & .0066926 & & & .0021973 & .0044466 & .00 \\
\hline AabbDdEE ... & $\cdots$ & .0039063 & .0089264 & .0120680 & --- & .0030864 & .0066926 & .0084717 & -.... & .0021973 & .0044466 & .0051360 \\
\hline aaBBDdEe -..- &.--- & .0039063 & .0089264 & .0120680 & & .0030864 & .0066926 & .0 & & .0021973 & .0044466 & .0051360 \\
\hline aaBbDDEe _-- & ----- & .0039063 & .0089264 & .0120680 & & .0030864 & .0066926 & & --- & .0021973 & .0044466 & 51360 \\
\hline aaBbDdEE -.-- & --- & .0039063 & .0089264 & .0120680 & $\ldots-$ & .0030864 & .0066926 & .0084717 & $-\cdots$ & .0021973 & .0044466 & .0051360 \\
\hline ARbiddee ... & -..- & & & &.--- & & & & - . . & & & \\
\hline AbbDdee _--- & ---- & .0019531 & .004 & & ---- & .0015432 & & & ---- & 986 & & \\
\hline AbbddEe --- & --- & .0019531 & .0043259 & & & .0015432 & .0044019 & & & & & \\
\hline AaBBddee -..- & ---- & .0019531 & .0043259 & .005 & - - & .0015432 & .004 & & --- & 0986 & 544 & .00 \\
\hline AabbDDee --- & ---- & .0019531 & .0043259 & .005 & & .0015432 & .00 & .00 & & .0010986 & .0034544 & .0052060 \\
\hline AabbddEE ...- & ---- & .0019531 & .0043259 & .00 & $-\cdots$ & .0015432 & $\$ 4019$ & & $-\cdots$ & & & \\
\hline aaBBDdee --- & $\cdots$ & .0019531 & .0043259 & .0059289 & $-\cdots$ & .0015432 & .0044019 & & $-\ldots$ & 10986 & & 52060 \\
\hline & ---- & .0019531 & .0043259 & .0059289 & & .0015432 & & & & & & \\
\hline aaBbDDee --- & ---- & .0019531 & .0043259 & .0059289 & $\ldots$ & .0015432 & .0044019 & .00 & ---- & .0010986 & .0034544 & .0052060 \\
\hline a aBbddEE -.. & $-\cdots$ & .0019531 & .0043259 & & $-\cdots$ & .0015432 & 44019 & & $-\ldots$ & .0010986 & 34544 & .0052060 \\
\hline aabbDDEe ..- & $\cdots$ & & & & & & & & $\cdots-$ & & & \\
\hline aabbDdEE --- & --- & .0019531 & .0043259 & .0059289 & $\cdots$ & .0015432 & .0044019 & .0066440 & --- & .0010986 & .0034544 & .0052060 \\
\hline $\mathrm{Ee}--.50$ & 000000 & & & & 1444443 & & & & 3750000 & & & \\
\hline ee --- & ---- & .0009766 & .0027809 & .003577 & & 97716 & 7629 & & $\cdots$ & & & 4688 \\
\hline ABBddee -..- & $\cdots$ & .0009766 & .0027809 & & & & & & -...- & & & \\
\hline aabbDDee --. & $-\cdots$ & .0009766 & .0027809 & .0035778 & $-\cdots$ & .0007716 & .0037629 & & --- & 105493 & & .0064688 \\
\hline aabbddEE ...- & . & .009766 & .0027809 & .0035778 & 然 & .0007716 & .0037629 & .0064009 & $\ldots$ & .0005493 & .0033904 & .0064688 \\
\hline e -.- & ---- & & & & $\cdots$ & & & & & & & \\
\hline AaBbddEe -.- & $\cdots$ & & & & & & & & $\cdots$ & & & \\
\hline AabbDdEe --- & --- & .0234375 & & & $\cdots-$ & & & & $-\cdots$ & & .0317391 & .0304671 \\
\hline aaBbDJEe -..- & ---- & .0234375 & .0304871 & .0311923 & ---- & .0308642 & .0361261 & .0363928 & $-\cdots-$ & .0307617 & .0317391 & .0304671 \\
\hline aBbddee -..- & --- & & & & -... & & & & $\cdots$ & & & \\
\hline AabbDdee --- & $\cdots$ & .0195313 & & & & & & & $-=$ & & & \\
\hline abbddBe -..- & $\cdots$ & .0195313 & .0191803 & & $-\cdots$ & .0277778 & & & --- & & & .0383573 \\
\hline & ---- & .0195313 & .0191803 & .018263 & & .0277778 & .0320270 & .03528 & ---- & .0285645 & .0342641 & .0383573 \\
\hline aaBbddEe ... &.--- & .0195313 & .0191803 & .0182631 & --- & .0277778 & & & --- & & & \\
\hline aabbDdEe -- - & $-\cdots$ & .0195313 & .0191803 & .0182631 & $\cdots$ & .0277778 & .0320270 & .0352809 & --- & .0285645 & .0342641 & .0383573 \\
\hline e... & $\cdots$ & .0175 & & & --- & & & & $=\ldots$ & & & \\
\hline & $\cdots$ & & & .013405 & ---- & .0262346 & .0418890 & & -... & .0274658 & .0539486 & .0631715 \\
\hline aabbDdee ...- & -..-- & .0175781 & .0175095 & .0134057 & $\ldots$ & .0262346 & & & & & .0539486 & .0631715 \\
\hline aabbddEe --- & & .0175781 & .0175095 & .0134057 & $-\ldots$ & .0262346 & .0418890 & .0433133 & --- & .0274658 & .0539486 & .0631715 \\
\hline & & & 5612 & $5 / 6+2$ & 144444 & 938 & $.1163 / 8$ & .0732723 & 025000 & .3433228 & .2146069 & 134 \\
\hline
\end{tabular}


at.e 32. - Relat:ve irecuenzes of genotypes when native population (AB:DOEE) is overflooded once in parental generation by $\underline{X}$ release insects abbddeel for every native insect--Continued

\begin{tabular}{|c|c|c|c|c|c|c|c|c|c|c|c|c|}
\hline \multirow{2}{*}{ vezotype } & & $\underline{x}$ & $\Rightarrow \quad$ & & & $\underline{x}=$ & 5 & & & $\underline{x}$ & 10 & \\
\hline & 2 & $\bar{z}$ & E. & $5_{4}$ & $F_{1}$ & 2 & 3 & $F_{4}$ & $F_{1}$ & $F_{2}$ & $\mathrm{~F}_{3}$ & $\mathrm{~F}_{4}$ \\
\hline AASBDDEE S. & $0=00000$ & 0.0036000 & 0.0004368 & 0.0000777 & 0.0277778 & 0.0020375 & 0.0002072 & 0.0000311 & 0.00826450 & 0.0003458 & 0.0000208 & 0.0000019 \\
\hline MSB $\div-$ & $\ldots$. & $.002-000$ & $.00071+5$ & .0002236 & -.... & .0015673 & .0003853 & .0001024 & -.... & .0003842 & .0000552 & .0000089 \\
\hline A3332dEE -- & ......- & $.002-000$ & $.00071 \omega 8$ & $.000=236$ & -... & .0015673 & .0003853 & .0001024 &.--- & .0003842 & .0000552 & .0000089 \\
\hline AHB -DEE - - & -... & $.002=000$ & .00071 .68 & .000223 .6 & ..... & .0015673 & .0003853 & .0001024 & & .0003842 & .0000552 & .0000089 \\
\hline 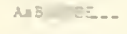 & -... & $.002 \approx 000$ & $.00071+8$ & .0002236 & --- & .0015673 & .0003853 & .0001024 & $\ldots$ & .0003842 & .0000552 & .0000089 \\
\hline AABBDBee.- & ..... & $.000-000$ & .0002924 & .0001610 & ---- & .0003014 & .0001791 & .0000842 & & .0001067 & .0000366 & .0000106 \\
\hline 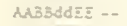 & -... & $.000 \sim 000$ & .000292 & .0001010 & -...- & .000301 & .0001791 & .0000842 & $-\ldots-$ & .0001067 & .0000366 & .0000106 \\
\hline$a b b=25--$ & -..- & $.000-000$ & .000292 & .0001610 & $\ldots$ & .0003014 & .0001791 & .0000842 & -...- & .0001067 & .0000366 & .0000106 \\
\hline a $\triangle B$ B $\supset E E$ - - & $-\ldots-$ & $.000-000$ & .000292 & .0001610 & $-\cdots$ & .0003014 & .0001791 & .0000842 & $\ldots$ & .0001067 & .0000366 & .0000106 \\
\hline MBBDdZ:-- & - & .0032000 & .0016256 & .0007769 & -.... & .0021701 & .0009726 & .0004051 & & .0005976 & 0001851 & .0000505 \\
\hline Ee-- & $-\cdots$ & .0032000 & .0016256 & .0007769 & ---- & .0021701 & .0009726 & .0004051 & - - & .0005976 & .0001851 & .0000505 \\
\hline $4=25-$ & $-\ldots$ & .0032000 & .0016256 & .0007769 & $-\cdots$ & .0021701 & .0009726 & .0004051 & -...- & .0005976 & & .0000505 \\
\hline נét- & $-\ldots+$ & .0032000 & .0016256 & .0007769 & $\ldots$ & .0021701 & .0009726 & $.000<051$ & 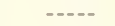 & .0005976 & .0001851 & .0000505 \\
\hline dEe- & $\ldots$ & .0032000 & .0016256 & .0007769 & -..- & .0021701 & .0009726 & .0004051 & -.... & .0005976 & .0001851 & .0000505 \\
\hline$B b=\cong-$ & $-\cdots$ & .0032000 & .0016256 & .0007769 & - - & .0021701 & .0009726 & .0004051 & - - & .0005976 & & .000 \\
\hline$e^{-}$ & $\ldots$ & .0008000 & .0008000 & .0006550 & $=\ldots$ & .0006028 & .0005712 & .0003892 & -.... & .0002134 & .0001484 & .0000698 \\
\hline Ee- & $\ldots$ & .0003000 & .0008516 & $.0 \times 65$ & -...-- & .0006028 & .0005712 & & ---- & .0002134 & .0001484 & .0000 \\
\hline Dee-- & --- & .0008000 & .0008516 & .0000550 & .... & .0006028 & .0005712 & .0003892 & $\ldots$ & .0002134 & .0001484 & .0000698 \\
\hline$E=-$ & ---- & .0003000 & .0008516 & .0006550 & $\ldots$ & .0006028 & .0005712 & .00038 & -... & .0002134 & & .00 \\
\hline DEe- & $\ldots$ & .0003000 & .0008516 & .0005550 & $-\ldots$ & .0006028 & .0005712 & .0003892 & $-\cdots$ & .0002134 & .0001484 & .0000698 \\
\hline ANbB:dEE-- & $\cdots$ & .0009000 & .0008516 & .0006550 & $\ldots$ & .0006028 & .0005712 & .0003892 & $\ldots . .$. & .0002134 & .0001484 & .0000698 \\
\hline $\mathrm{ces}^{--}$ & $\ldots$ & .0008000 & .0008516 & .0006550 & -...- & .0006028 & .0005712 & .000 & -..- & .0002134 & 484 & .00 \\
\hline BBDCEE=- & $\ldots=-$ & .0008000 & .0008516 & .0006550 & $\ldots$ & .0006028 & .0005712 & .00038 & $-\ldots$ & .0002134 & 484 & .00 \\
\hline oes-- & -... & .0008000 & $.000 \$ 516$ & .0006550 & --.-- & .0006028 & .0005712 & .000389 & -..-- & .0002134 & .0001484 & .0000698 \\
\hline DEe-- & ---- & .0005000 & $.000 \leqslant 516$ & .0006550 & $-\ldots-$ & .0006028 & .0005712 & .0003 & ---- & .0002134 & & .00 \\
\hline 3DdEE-- & $\ldots$ & .0008000 & .0008516 & .0006150 & -.... & .0006028 & .0005712 & .00038 & ..... & .0002134 & 01484 & .0000698 \\
\hline aABDTDEE- & -.. & .0008000 & .0008516 & .0006550 & $-\ldots$ & .0006028 & .0005712 & .0003892 & 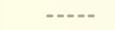 & .0002134 & .0001484 & .000 \\
\hline$M B B D D E e^{--}$ & -... & .0045000 & .0047763 & $.003299 \mathrm{C}$ & -... & .0033758 & .0030984 & .0019481 & -... & .0010245 & .0007317 & .0003396 \\
\hline Ee- & $\ldots$ & .0048000 & .0047763 & .0032990 & -.... & & & & & .0010245 & & .000 \\
\hline bDDCe=- & & .0048000 & .0047743 & .0032990 & $\ldots$ & .0033758 & .0030984 & .001 & $\ldots+\ldots$ & .0010245 & .0007317 & .0003396 \\
\hline AMBbDdEE- & $\ldots$ & .0048000 & $.00<77 \div 3$ & .0032990 & -... & .0033758 & .0030984 & .0019481 & $-\ldots$ & .0010245 & .0007317 & .0003396 \\
\hline Misddee - & -...- & $.000<000$ & .0006200 & .0006663 & - & .0003014 & .0004554 & .0004696 & & 01067 & 01504 & .0001147 \\
\hline iet- & $-\ldots$ & .0006000 & .0006200 & .0006 & $\ldots$ & .0003014 & .00 & .000 & .. & .0001067 & & .00 \\
\hline SddEE - & -- & .0004000 & .0006200 & .0006 & 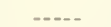 & .0003014 & .00 & .00 & & .0001067 & & \\
\hline BBDDee-- & & $.000-000$ & .0006200 & .0006663 & $\ldots .$. & .0003014 & .0004556 & .000 & $\ldots$ & .0001067 & .0001504 & .0001147 \\
\hline aA3BddEE - &.- & $.000<000$ & .0006200 & .0006663 & $\ldots$ & .0003014 & .0004554 & .006 & ..... & .0001067 & .0001504 & .0001147 \\
\hline 2Abb DEE-- & & $.000-000$ & .0006200 & .0006663 & $\ldots$ & .0003014 & .0004554 & .0004496 & -.... & .0001067 & .0001504 & .0001147 \\
\hline$e^{--}$ & $\ldots$ & .0016000 & .0030560 & .0032531 & -... & .0012056 & .0021983 & .002 & --.-- & .0004269 & .0006808 & .0005399 \\
\hline$e^{--}$ & $-\cdots$ & 016000 & .0030560 & .0032531 & $-\ldots$ & .0012056 & .0021983 & .002 & ---+ & .0004269 & & .0005399 \\
\hline $12 e^{--}$ & -- & $.001 E 000$ & .0030550 & .0032531 &.- & .0012056 & & .00 & $-\ldots$ & 04269 & & 5399 \\
\hline Ddee -- & -- & .0016000 & .0030560 & .0032531 & $\ldots$ & .0012056 & .0021983 & .00218 & ..... & .0004269 & & 5399 \\
\hline EEe-- & $-\cdots$ & .0016000 & .0030560 & .0032531 & ---- & .0012056 & .0021983 & .0021831 & ---- & .0004269 & .0006808 & .0005399 \\
\hline $\mathrm{ee}^{--}$ & $-\cdots$ & .0016000 & .0030560 & .0032531 & ---- & .0012056 & .0021983 & .00218 & -.-.- & .0004269 & .00 & .0005399 \\
\hline$b d d E E=-$ & $=-\infty$ & .0016000 & .0030560 & .0032531 & $-\ldots$ & .0012056 & .0021983 & .0021831 & $-\ldots$ & .0004269 & .0006808 & 5399 \\
\hline$E_{e}^{--}$ & $-\cdots$ & .0015000 & .0030560 & .0032531 & $-\cdots$ & .0012056 & .0021983 & .00218 & -..- & .0004269 & .0006808 & .0005399 \\
\hline$E \varepsilon=-$ & $\ldots$ & .0015000 & .0030560 & .0032531 & -.... & .0012056 & .0021983 & .00 & $\ldots .$. & .0004269 & & 05399 \\
\hline dEe-- & 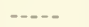 & .0016000 & .0030560 & .0032531 & -... & .0012056 & .0021983 & .0021831 & -...- & .0004269 & .0006808 & .0005399 \\
\hline DOEe -- & $\ldots$ & .0016000 & .0030560 & .0032531 & $\ldots$ & .0012056 & .0021983 & .00218 & $\ldots$ & .0004269 & .0006808 & .0005399 \\
\hline A $\triangle 3 b D d E E$-- & $\ldots$ & .0016000 & .0030560 & .0032531 & $-\ldots$ & .0012056 & .0021983 & .0021831 & -... & .0004269 & .0006808 & .0005399 \\
\hline Aribddee-- & & & & & & & & & & .0002134 & 17703 & \\
\hline Ahbb Jeec- - & & .0008200 & 0026464 & & & .0006028 & 0531 & .002 & ..... & .0002134 & .0007703 & .0010204 \\
\hline MubddEE_- & $\ldots$ & .00 .8200 & .0026444 & .0039 & ---- & .0006028 & & & $\ldots$ & .0002134 & 7703 & 10204 \\
\hline AaBBddee.. & & .0008000 & .0026444 & .0039074 & $-\ldots+$ & .0006028 & .0020531 & .00 & 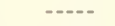 & .0002134 & .0007703 & .0010204 \\
\hline dee -- & & .0008000 & .0026444 & .0039074 & --- & .0006028 & .0020531 & .0029661 & $-\ldots$ & .0002134 & .0007703 & .0010204 \\
\hline $\begin{array}{l}\text { dEE_-- } \\
\text { dee-- }\end{array}$ & & 0008000 & .00264464 & .0039074 & 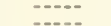 & .0006028 & .0020531 & & -...- & .0002134 & .00 & .0010204 \\
\hline a.BBAdEe- - & $\ldots$ & 0008000 & .0026444 & .0039074 & & .0006028 & .0020531 & .00296 & & .0002134 & .0007703 & .0010204 \\
\hline aa Sb DDee- - & $-\cdots$ & .0008000 & .0026444 & .0039074 & $\ldots$ & .0006028 & .0020531 & .0029661 & $\ldots$ & .0002134 & .0007703 & .0010204 \\
\hline$b d d E z-$ & $\ldots$ & .0008000 & .0026644 & .0039074 & $\ldots$ & .0006028 & & .0029661 & $\ldots$ & .0002134 & .0007703 & .0010204 \\
\hline a.abbDoce- & ..... & .0008000 & .00264644 & .0039074 & -..-- & .0006028 & .0020531 & .0029661 & ..... & .0002134 & .0007703 & .0010204 \\
\hline aAbbDdEE- & --- & .0008900 & .0025444 & .0039074 & $-\ldots$ & .0006028 & .0006028 & .0029661 & -... & .0002134 & .0007703 & .0010204 \\
\hline & & .0009200 & .0026444 & .0039 & & & .0006028 & .002 & & .0002134 & .0007703 & .0010204 \\
\hline & 3200000 & .0848000 & .0339282 & 1896 & .2777777 & & & & 1652892 & & & \\
\hline & $\cdots . .-$ & .0004000 & & & ..... & & & & & 1067 & & \\
\hline azBBddear- & .... & .0004000 & .0028196 & .0057286 & -.... & .0003014 & & & 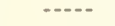 & & & .0022690 \\
\hline asbbDDee_ & & .0004000 & .0028196 & .0057286 & -1 & .0003014 & .0023141 & .004891 & ..... & .0001067 & & .0022690 \\
\hline aabbddEE- & :ב-:- & .0004000 & .0028196 & .0057286 & 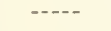 & .0003014 & .0023141 & .0048919 & -... & .0001067 & .0009865 & .0022690 \\
\hline dasbodee. - & & 283000 & .0267005 & & $\ldots+$ & & & & ..... & & & \\
\hline & & .0288000 & .0267055 & .0239735 & --.. & & & & & 79291 & & \\
\hline EAbBDdEe- & $\ldots$ & .0288000 & .0267055 & .0239735 &..-- & .0265239 & .0225292 & & -.... & 99291 & 540 & .0078245 \\
\hline aaBbDdEer- & $\ldots$ & .0283000 & .0267055 & .0239735 & - & .0265239 & .0225292 & .0190071 & $\ldots$ & .0179291 & .0116540 & .0078245 \\
\hline As Zboddee -- & $-\cdots$ & & & & ..... & & & & $\ldots+$ & & & \\
\hline AabbDdea- - & & .0272000 & .0329744 & .0363 & $-\ldots$ & & & & & .0175022 & .0209065 & \\
\hline AabbddEe - & & .0272000 & .0329744 & .0363824 & $\ldots . .$. & .0253183 & & & $\ldots$ & .0175022 & .0209065 & .0201927 \\
\hline aaBbDdea- & ---- & .0272000 & .0329744 & & $-\ldots$ & .0253183 & .03073 & & $\ldots$ & 75022 & & .0201927 \\
\hline aaBbddzo- & & .0272000 & .0329744 & .0363824 & $-\cdots$ & .0253183 & .0307316 & .0331386 & & .0175022 & .0209065 & .0201927 \\
\hline aabbDdEe - - & - & .0272000 & .0329746 & .0363824 & -...- & .0253183 & .0307316 & .0331386 & - & .0175022 & .0209065 & .0201927 \\
\hline Aabbddee =- & -... & .0264000 & .0582932 & & $\cdots$ & & & & -... & .01728 & & \\
\hline & $-\cdots$ & .0264000 & .0582932 & & & & & .07703 & & & 84957 & .0696211 \\
\hline aabbDdee -- & $\ldots \ldots$ & .0264000 & .0582932 & .0730857 & $\cdots$ & .0247154 & .0588434 & .0770364 & $-\ldots$ & .0172888 & .0484957 & .0696211 \\
\hline datovase - - & - & .0264000 & .0582932 & .0730857 & 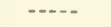 & .0247154 & .0588434 & .0770364 & 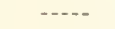 & .0172888 & .0484957 & .0696211 \\
\hline aabbddee - . & 6400000 & .4355999 & .3012911 & .2331079 & .6944443 & .5066671 & .3740759 & .3032902 & .8264463 & .7001955 & .5960293 & .5340672 \\
\hline
\end{tabular}


Table 32.--Relative frequencies of genotypes when native population (AABBDDEE) is overflooded once in parental generation by $\underline{X}$ release insects (aabbddee) for every native insect--Continued

\begin{tabular}{|c|c|c|c|c|c|c|c|c|c|c|c|c|}
\hline \multirow{2}{*}{ Genotype } & & $\underline{x}=$ & 20 & & & $\underline{X}$ & 50 & & & $\underline{x}$ & 00 & \\
\hline & $\overline{\mathrm{E}}_{1}$ & $\mathrm{~F}_{2}$ & $F_{3}$ & $\mathrm{~F}_{4}$ & $F_{1}$ & $\mathrm{~F}_{2}$ & $\mathrm{~F}_{3}$ & $\mathrm{~F}_{4}$ & $\mathrm{~F}_{1}$ & $\mathrm{~F}_{2}$ & $\mathrm{~F}_{3}$ & $\mathrm{~F}_{4}$ \\
\hline AABBDDEE_ 0.0 & 0022676 & 0.0000630 & 0.0000024 & 0.0000001 & 0003845 & 0.0000078 & 0.0000002 & 0.0000000 & 0000980 & 0.0000018 & 0.0000000 & 0.0000000 \\
\hline AABBDDEe -- & ---- & .0000900 & .0000085 & .0000009 & -... & .0000134 & .0000009 & .0000001 & $\cdots$ & .0000032 & .0000002 & .0000000 \\
\hline AABBDdEE -- & -..- & .0000900 & .0000085 & .0000009 & - - & .0000134 & .0000009 & .0000001 & ---- & .0000032 & .0000002 & .0000000 \\
\hline AABBDDEE -- & $\cdots$ & .0000900 & .0000085 & .0000009 & --- & .0000134 & .0000009 & .0000001 & & .0000032 & .0000002 & .0000000 \\
\hline AaBBDDEE -- & ---- & .0000900 & .0000085 & .0000009 & ---- & .0000134 & .0000009 & .0000001 & & .0000032 & .0000002 & .0000000 \\
\hline AABB DDee -- & ---- & .0000321 & .0000075 & .0000014 & $\cdots-$ & .0000058 & .0000010 & .0000001 & & .0000015 & .0000002 & .0000000 \\
\hline AABBddEE -- & ----- & .0000321 & .0000075 & .0000014 & -.... & .0000058 & .0000010 & .0000001 & -..- & .0000015 & .0000002 & .0000000 \\
\hline AbDDDEE -- & & .0000321 & .0000075 & .0000014 & ---- & .0000058 & .0000010 & .0000001 & & .0000015 & .0000002 & .0000000 \\
\hline aaBBDDEE -- & $-\ldots$ & .0000321 & .0000075 & .0000014 & $-\cdots$ & .0000058 & .0000010 & .0000001 & & .0000015 & .0000002 & .0000000 \\
\hline Ee-- & ---- & .0001543 & .0000354 & .0000066 & & .0000249 & .0000044 & .00000 & & .0000062 & .0000010 & .0000001 \\
\hline -- & ---- & .0001543 & .0000354 & .0000066 & -... & .0000249 & .0000044 & .0000006 & & .0000062 & .0000010 & .0000001 \\
\hline EE -- & ---- & .0001543 & .0000354 & .0000066 & $\cdots$ & .0000249 & .0000044 & .0000006 & & .0000062 & .0000010 & .0000001 \\
\hline e -- & ---- & .0001543 & .0000354 & .0000066 & $\cdots$ & .0000249 & .0000044 & .0000006 & & .0000062 & .0000010 & .0000001 \\
\hline EE -- & -..- & .0001543 & .0000354 & .0000066 & $-\cdots$ & .0000249 & .0000044 & .0000006 & & .0000062 & .0000010 & .0000001 \\
\hline AaBbDDEE -- & $-\cdots$ & .0001543 & .0000354 & .0000066 & ---- & .0000249 & .0000 & .0000 & --- & .0000062 & .0000 & \\
\hline lee -- & & .0000643 & .0000360 & .0000124 & ---- & .0000115 & .0000 & .0000 & & .0000030 & .0000 & .0000 \\
\hline e -- & & .0000643 & .000 & & & .0000115 & .0000 & & & .0000030 & .0000 & .0000 \\
\hline e -- & ---- & .0000643 & .000 & .0000 & $-\ldots$ & .0000115 & .0000 & .00 & & .0000030 & .0000 & .0000003 \\
\hline E -- & $-\ldots--$ & .0000643 & .0000 & .0000 & ----- & .0000115 & .0000 & .00 & & .0000030 & .0000 & .0000003 \\
\hline Ee -- & & .0000643 & .0000360 & & ---- & .0000115 & .0000 & & & .0000030 & .0000 & .0000003 \\
\hline$E--$ & --- & .0000643 & .0000360 & .0000124 & & .0000115 & .00000 & & & .0000030 & .0000 & .0000003 \\
\hline e -- & ----- & .0000643 & .0000360 & .0000 & --- & .0000115 & .0000 & .0000 & & .0000030 & .0000 & .0000003 \\
\hline EE -- & & .0000643 & .0000360 & & & .0000115 & .0000 & & & .00000 & .0000 & .0000003 \\
\hline EE - & --- & .0000643 & .0000360 & .0000124 & & .0000115 & .0000055 & .0000 & & .0000030 & .000 & .000 \\
\hline Ee -- & & .0000643 & .000 & & & .0000115 & .0000 & & & .0000 & .000 & .0000 \\
\hline EE -- & & .0000643 & .000 & & & .0000115 & .0000 & & & .0000 & .000 & .0000003 \\
\hline EE -- & & .0000643 & .0000360 & .0000124 & $-\cdots$ & .0000115 & .0000 & .000 & & .000 & .000 & .000 \\
\hline & & .0002828 & .0001643 & .0000577 & & .0000480 & .0000235 & & & .0000122 & .0000 & .0000013 \\
\hline Se -- & & .0002828 & .000 & & & .0000480 & .0000 & & & .00 & & \\
\hline Ee .- & & .0002828 & .0001 & & & .0000480 & .0000 & .000 & & .0000122 & .000 & .0000013 \\
\hline AEE .. & & .0002828 & .0001643 & .0000577 & & .0000480 & .0000235 & .000 & & .0000122 & .000 & .0000013 \\
\hline ABBddee -- & & .0000321 & .0000432 & & & .0000058 & .0000075 & & & .0000015 & .000 & .0000010 \\
\hline ee -- & & .0000321 & .000 & .00 & & .0000058 & .0000 & & & .000 & .000 & 010 \\
\hline EE -- & & .0000321 & .000 & .00 & & .0000058 & .0000 & & & .0000 & .000 & 010 \\
\hline ee -- & & .0000321 & .000 & & & .0000058 & .0000 & & & .0000 & .000 & .0000010 \\
\hline EE -- & & .0000321 & .0000432 & .0000 & - & .0000058 & .0000075 & .000 & & .0000015 & .000 & .0000010 \\
\hline aаbЬDDEE -- & & .0000321 & .0000432 & .0000269 & ---- & .0000058 & .0000075 & .0000040 & & .0000015 & .0000019 & .0000010 \\
\hline ee $=-$ & -- & .0001285 & .000 & & & .0000231 & .0000 & & & & & \\
\hline Ee -- & & .0001285 & .000 & .000 & & .0000231 & .000 & & & .000 & .000 & .000 \\
\hline $\mathrm{Ee} .-$ & & .0001285 & .000 & .00 & ---- & .0000231 & .000 & & & .0000 & .000 & .0000040 \\
\hline ee -- & -- & .0001285 & .000 & .000 & -...- & .0000231 & .000 & & & .0000 & .000 & 040 \\
\hline Ee -- & -- & .0001285 & .0001858 & .00012 & $\cdots$ & .0000231 & .000 & & & .000 & .000 & \\
\hline ee -- & --- & .000 & .000 & & ---- & .0000231 & .0000 & .000 & $-\ldots$ & .0000060 & .00000 & .0000040 \\
\hline HEE -- & ---- & .0001285 & .000 & .00 & ---- & .0000231 & .000 & & & .0000 & .000 & .00 \\
\hline DEe -- & ----- & .0001285 & .000 & .000 & - & .0000231 & .000 & & & .0000 & .000 & \\
\hline dEE -- & & .0001285 & .000 & .0001209 & $-\cdots$ & .0000231 & .0000310 & .00 & & .0000 & .000 & .00 \\
\hline DdEe -- & ---- & .0001285 & .000 & .0001209 & -.... & .0000231 & .000 & & & .000 & .0000 & .000 \\
\hline DDEe .. & $\ldots$ & .0001285 & .000 & .0001 & -- & .0000231 & .000 & & $\ldots$ & .0000 & .0000 & .000 \\
\hline aaBbDdEE .. & & .0001285 & .0001858 & .0001209 & ---- & .0000231 & .0000310 & .0000169 & & .0000060 & .0000078 & .0000040 \\
\hline lee =- & & .000 & & & -... & 115 & & & & & & \\
\hline dee -- & -.. & .0000643 & .000 & & ----- & .0000115 & .000 & & & & & .0000126 \\
\hline IdEe -- &.- & .0000643 & .000 & & & & .0000434 & & - & & & \\
\hline & -... & .0000643 & .000 & & - & 00115 & .000 & & & & & \\
\hline dee .. & & & .000 & & $\cdots$ & 00115 & .000 & & & & & 126 \\
\hline DddEE .- & & .0000643 & .0002382 & & & & & & & & & \\
\hline Ddee -- & $-\ldots$ & .0000643 & .0002382 & .00 & ---- & .0000115 & .0000434 & & - & & & 126 \\
\hline ddEe -- & & & .0002382 & & $\cdots$ & .0000115 & .0000434 & & & & & \\
\hline Dee -- & $\ldots$ & .0000643 & .0002 & & $-\cdots$ & .0000115 & & & ---- & & & \\
\hline ddEE -- & & .0000643 & .000 & 2916 & -..- & .0000115 & .0000434 & & & .00 & & 126 \\
\hline DDEe -- & & .0000643 & .0002382 & & ---- & .0000115 & .00 & & & & & \\
\hline aabbDdEE -- & & .0000643 & $.00023 \mathrm{~B} 2$ & .0002916 & $-\cdots$ & .0000115 & .0000434 & .0000497 & & .0000030 & .0000114 & \\
\hline e.-. & 0907029 & & & & $03 B 4467$ & & & & 196059 & & & \\
\hline ee .- & $\cdots$ & & & & $-\ldots$ & & & & & & & \\
\hline ee -- & $-\infty$ & .0000 & .000 & & --- & & & & $\cdots$ & & & \\
\hline Dee -- & & & & & & & & & $\cdots$ & & & \\
\hline aabbddEE -- & & .0000321 & .0003287 & .0007908 & $\cdots$ & .0000058 & .0000629 & .0001557 & $\cdots$ & .0000015 & .0000167 & .0000418 \\
\hline ee -- & --- & & & & $--\cdots$ & & & & - & & & \\
\hline & $-\cdots$ & .0105488 & & & ---- & 46654 & & & & & & 3443 \\
\hline He -.. & - & .0105488 & .0055177 & & ---- & & & & & & & 3443 \\
\hline & & .0105488 & .0055177 & .0029070 & & .0046654 & .0020399 & .0008209 & & .0024145 & .0009817 & .0003443 \\
\hline-- & & & & & & & & & & & & \\
\hline ee -- & ---- & & & & -...- & & & & & & & \\
\hline & $-\cdots$ & .0104123 & .0121626 & & --- & .0046423 & .00 & & -...- & .002 & .0027 & .0019754 \\
\hline & & & & & & & & & & & & \\
\hline & --- & .0104123 & .0121626 & & -...- & & & & 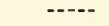 & & .0027341 & .0019754 \\
\hline aabbDdEe -- & $-\cdots$ & .0104123 & .0121626 & .0104074 & -2 & .0046423 & .0053136 & .0040417 & $\cdots$ & .0024085 & .0027341 & .0019754 \\
\hline & $\ldots$ & & & & --- & & & & & & & \\
\hline & & & .0317370 & & 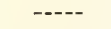 & & & & & & & \\
\hline ee -- & $-\cdots$ & .0103481 & .0317370 & & ---- & & & & $=--$ & & .0079610 & .0125095 \\
\hline aabbddEe -. & $-\ldots$ & .0103481 & .0317470 & .0478775 & $\cdots$ & .0046308 & .0150326 & .0233767 & $\cdots$ & .0024055 & .0079610 & .0125095 \\
\hline tboddee $=$. & 0289 & 330183 & 665649 & .7246644 & 9611688 & .9284704 & .8975079 & .8772606 & 9802959 & .9633843 & .9470770 & .9362800 \\
\hline
\end{tabular}


Tasle 32.--Selative frequencies of genotypes when native population (AABBODEE) is overflooded once in parental generation by $\mathrm{X}$

release insects asbbdiee) for every native insect.-Continued

\begin{tabular}{|c|c|c|c|c|c|c|c|c|c|c|c|c|}
\hline \multirow[b]{2}{*}{ Genotype } & \multicolumn{4}{|c|}{$I=200$} & \multicolumn{4}{|c|}{$\underline{x}=500$} & \multicolumn{4}{|c|}{$x=1,000$} \\
\hline & $F_{1}$ & $F_{2}$ & $\mathrm{~F}_{3}$ & $F_{\dot{4}}$ & $F_{1}$ & $\mathrm{~F}_{2}$ & $F_{3}$ & $F_{4}$ & $F_{1}$ & $\mathrm{~F}_{2}$ & $\mathrm{~F}_{3}$ & $E_{4}$ \\
\hline 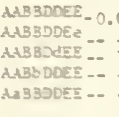 & $\begin{array}{l}0000 z-8 \\
0 \ldots- \\
\ldots \ldots\end{array}$ & $\begin{array}{l}0.0000006 \\
.0000008 \\
.0000008 \\
.0000008 \\
.0000008\end{array}$ & $\begin{array}{l}.00000000 \\
.0000000 \\
.0000000 \\
.0000000 \\
.0000000\end{array}$ & $\begin{array}{l}0.0000000 \\
.0000000 \\
.0000000 \\
.0000000 \\
.0000000\end{array}$ & \begin{tabular}{c}
0,0000040 \\
$\ldots . .$. \\
\hdashline$\ldots$ \\
\hdashline..-
\end{tabular} & $\begin{array}{l}0.00000010 \\
.0000001 \\
.0000001 \\
.0000001 \\
.0000001\end{array}$ & $\begin{array}{l}.0000000 \\
.0000000 \\
.0000000 \\
.0000000 \\
.0000000\end{array}$ & $\begin{array}{r}0.0000000 \\
.0000000 \\
.0000000 \\
.0000000 \\
.0000000\end{array}$ & 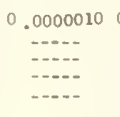 & $\begin{array}{l}0.0000000 \\
.0000000 \\
.0000000 \\
.0000000 \\
.0000000\end{array}$ & $\begin{array}{l}0.00000000 \\
.0000000 \\
.0000000 \\
.0000000 \\
.0000000\end{array}$ & $\begin{array}{r}0.0000000 \\
.0000000 \\
.0000000 \\
.0000000 \\
.0000000\end{array}$ \\
\hline $\begin{array}{l}\text { ABBDDEe -- } \\
\text { AABBddEE =- } \\
\text { ABbBODEE -- } \\
\text { A SBDDEE -- }\end{array}$ & $\begin{array}{l}-\ldots- \\
\cdots-\ldots \\
\cdots-\ldots\end{array}$ & $\begin{array}{l}0000004 \\
0000004 \\
0000004 \\
0000004\end{array}$ & $\begin{array}{l}.0000001 \\
.0000001 \\
.0000001 \\
.0000001\end{array}$ & $\begin{array}{l}.0000000 \\
.0000000 \\
.0000000 \\
.0000000\end{array}$ & $\ldots$ & $\begin{array}{l}.0000001 \\
.0000001 \\
.0000001 \\
.0000001\end{array}$ & $\begin{array}{l}.0000000 \\
.0000000 \\
.0000000 \\
.0000000\end{array}$ & $\begin{array}{l}.0000000 \\
.0000000 \\
.0000000 \\
.0000000\end{array}$ & $\begin{array}{l}-\cdots \\
-\cdots- \\
-\cdots-\end{array}$ & $\begin{array}{l}.0000000 \\
.0000000 \\
.0000000 \\
.0000000\end{array}$ & $\begin{array}{l}.0000000 \\
.0000000 \\
.0000000 \\
.0000000\end{array}$ & $\begin{array}{l}.0000000 \\
.0000000 \\
.0000000 \\
.0000000\end{array}$ \\
\hline & 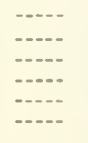 & $\begin{array}{l}.0000016 \\
.0000016 \\
.0000016 \\
.0000016 \\
.0000016 \\
.0000016\end{array}$ & $\begin{array}{l}.0000002 \\
.0000002 \\
.0000002 \\
.0000002 \\
.0000002 \\
.0000002\end{array}$ & $\begin{array}{l}.0000000 \\
.0000000 \\
.0000000 \\
.0000000 \\
.0000000 \\
.0000000\end{array}$ & $\begin{array}{l}\ldots . . . \\
\ldots \ldots \\
\cdots \ldots \\
\cdots \ldots\end{array}$ & $\begin{array}{l}.0000002 \\
.0000002 \\
.0000002 \\
.0000002 \\
.0000002 \\
.0000002\end{array}$ & $\begin{array}{l}.0000000 \\
.0000000 \\
.0000000 \\
.0000000 \\
.0000000 \\
.0000000\end{array}$ & $\begin{array}{l}.0000000 \\
.0000000 \\
.0000000 \\
.0000000 \\
.0000000 \\
.0000000\end{array}$ & 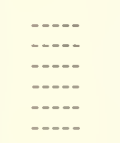 & $\begin{array}{l}.0000001 \\
.0000001 \\
.0000001 \\
.0000001 \\
.0000001 \\
.0000001\end{array}$ & $\begin{array}{l}.0000000 \\
.0000000 \\
.0000000 \\
.0000000 \\
.0000000 \\
.0000000\end{array}$ & $\begin{array}{l}.0000000 \\
.0000000 \\
.0000000 \\
.0000000 \\
.0000000 \\
.0000000\end{array}$ \\
\hline & 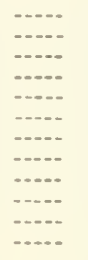 & $\begin{array}{l}.0000008 \\
.0000008 \\
.0000008 \\
.0000008 \\
.0000008 \\
.0000008 \\
.0000008 \\
.0000008 \\
.0000008 \\
.0000008 \\
.0000008 \\
.0000008\end{array}$ & $\begin{array}{l}.0000003 \\
.0000003 \\
.0000003 \\
.0000003 \\
.0000003 \\
.0000003 \\
.0000003 \\
.0000003 \\
.0000003 \\
.0000003 \\
.0000003 \\
.0000003\end{array}$ & $\begin{array}{l}.0000001 \\
.0000001 \\
.0000001 \\
.0000001 \\
.0000001 \\
.0000001 \\
.0000001 \\
.0000001 \\
.0000001 \\
.0000001 \\
.0000001 \\
.0000001\end{array}$ & 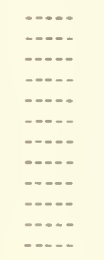 & $\begin{array}{l}.0000001 \\
.0000001 \\
.0000001 \\
.0000001 \\
.0000001 \\
.0000001 \\
.0000001 \\
.0000001 \\
.0000001 \\
.0000001 \\
.0000001 \\
.0000001\end{array}$ & $\begin{array}{l}.0000001 \\
.0000001 \\
.0000001 \\
.0000001 \\
.0000001 \\
.0000001 \\
.0000001 \\
.0000001 \\
.0000001 \\
.0000001 \\
.0000001 \\
.0000001\end{array}$ & $\begin{array}{l}.0000000 \\
.0000000 \\
.0000000 \\
.0000000 \\
.0000000 \\
.0000000 \\
.0000000 \\
.0000000 \\
.0000000 \\
.0000000 \\
.0000000 \\
.0000000\end{array}$ & 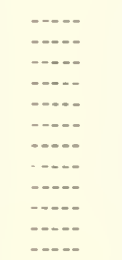 & $\begin{array}{l}.0000000 \\
.0000000 \\
.0000000 \\
.0000000 \\
.0000000 \\
.0000000 \\
.0000000 \\
.0000000 \\
.0000000 \\
.0000000 \\
.0000000 \\
.0000000\end{array}$ & & $\begin{array}{l}.0000000 \\
.0000000 \\
.0000000 \\
.0000000 \\
.0000000 \\
.0000000 \\
.0000000 \\
.0000000 \\
.0000000 \\
.0000000 \\
.0000000\end{array}$ \\
\hline & $\begin{array}{l}-\ldots- \\
-\cdots \\
-\cdots\end{array}$ & & $\begin{array}{l}.0000014 \\
.0000014 \\
.0000014 \\
.0000014\end{array}$ & $\begin{array}{l}.0000003 \\
.0000003 \\
.0000003 \\
.0000003\end{array}$ & $\begin{array}{l}-\ldots- \\
\cdots-\cdots \\
-\cdots\end{array}$ & & $\begin{array}{l}.0000002 \\
.0000002 \\
.0000002 \\
.0000002\end{array}$ & & $\begin{array}{l}-\ldots \\
\cdots-.- \\
-\cdots\end{array}$ & $\begin{array}{l}.0000001 \\
.0000001 \\
.0000001 \\
.0000001\end{array}$ & & $\begin{array}{l}0000 \\
0000 \\
0000\end{array}$ \\
\hline $\begin{array}{l}\text { A3Bddee -- } \\
\text { Abb ODee -- } \\
\text { Ab bddEE -- } \\
\text { abODee -- } \\
\text { aBBddEE -- } \\
\text { abb DDEE -- }\end{array}$ & $\begin{array}{l}-\ldots \\
\ldots \ldots \\
\ldots \ldots \\
\ldots \ldots \\
\ldots \ldots\end{array}$ & $\begin{array}{l}.00000046 \\
.00000046 \\
.0000004 \\
.0000004 \\
.0000004 \\
.0000004\end{array}$ & $\begin{array}{l}.0000005 \\
.0000005 \\
.0000005 \\
.0000005 \\
.0000005 \\
.0000005\end{array}$ & $\begin{array}{l}.0000002 \\
.0000002 \\
.0000002 \\
.0000002 \\
.0000002 \\
.0000002\end{array}$ & 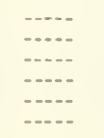 & $\begin{array}{l}.0000001 \\
.0000001 \\
.0000001 \\
.0000001 \\
.0000001 \\
.0000001\end{array}$ & $\begin{array}{l}.0000001 \\
.0000001 \\
.0000001 \\
.0000001 \\
.0000001 \\
.0000001\end{array}$ & $\begin{array}{l}.0000000 \\
.0000000 \\
.0000000 \\
.0000000 \\
.0000000 \\
.0000000\end{array}$ & 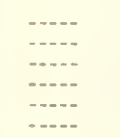 & $\begin{array}{l}.0000000 \\
.0010000 \\
.0000000 \\
.0000000 \\
.0000000 \\
.0000000\end{array}$ & $\begin{array}{l}.0000000 \\
.0000000 \\
.0000000 \\
.0000000 \\
.0000000 \\
.0000000\end{array}$ & \\
\hline 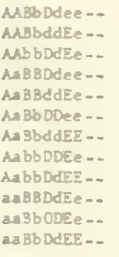 & 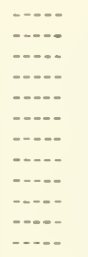 & $\begin{array}{l}.0000015 \\
.0000015 \\
.0000015 \\
.0000015 \\
.0000015 \\
.0000015 \\
.0000015 \\
.0000015 \\
.0000015 \\
.0000015 \\
.0000015 \\
.0000015\end{array}$ & $\begin{array}{l}.0000020 \\
.0000020 \\
.0000020 \\
.0000020 \\
.0000020 \\
.0000020 \\
.0000020 \\
.0000020 \\
.0000020 \\
.0000020 \\
.0000020 \\
.0000020\end{array}$ & $\begin{array}{l}.0000010 \\
.0000010 \\
.0000010 \\
.0000010 \\
.0000010 \\
.0000010 \\
.0000010 \\
.0000010 \\
.0000010 \\
.0000010 \\
.0000010 \\
.0000010\end{array}$ & 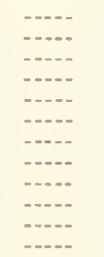 & $\begin{array}{l}.0000002 \\
.0000002 \\
.0000002 \\
.0000002 \\
.0000002 \\
.0000002 \\
.0000002 \\
.0000002 \\
.0000002 \\
.0000002 \\
.0000002 \\
.0000002\end{array}$ & $\begin{array}{l}.0000003 \\
.0000003 \\
.0000003 \\
.0000003 \\
.0000003 \\
.0000003 \\
.0000003 \\
.0000003 \\
.0000003 \\
.0000003 \\
.0000003 \\
.0000003\end{array}$ & & 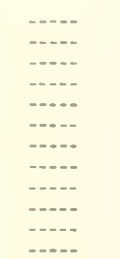 & $\begin{array}{l}.0000001 \\
.0000001 \\
.0000001 \\
.0000001 \\
.0000001 \\
.0000001 \\
.0000001 \\
.0000001 \\
.0000001 \\
.0000001 \\
.0000001 \\
.0000001\end{array}$ & $\begin{array}{l}.0000001 \\
.0000001 \\
.0000001 \\
.0000001 \\
.0000001 \\
.0000001 \\
.0000001 \\
.0000001 \\
.0000001 \\
.0000001 \\
.0000001\end{array}$ & \\
\hline 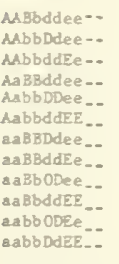 & 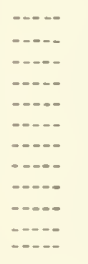 & $\begin{array}{l}.0000008 \\
.0000008 \\
.0000008 \\
.0000008 \\
.0000008 \\
.0000008 \\
.0000008 \\
.0000008 \\
.0000008 \\
.0000008 \\
.0000008 \\
.0000008\end{array}$ & $\begin{array}{l}.0000029 \\
.0000029 \\
.0000029 \\
.0000029 \\
.0000029 \\
.0000029 \\
.0000029 \\
.0000029 \\
.0000029 \\
.0000029 \\
.0000029 \\
.0000029\end{array}$ & $\begin{array}{l}.0000032 \\
.0000032 \\
.0000032 \\
.0000032 \\
.0000032 \\
.0000032 \\
.0000032 \\
.0000032 \\
.0000032 \\
.0000032 \\
.0000032 \\
.0000032\end{array}$ & 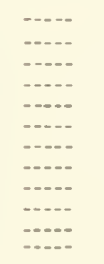 & $\begin{array}{l}.0000001 \\
.0000001 \\
.0000001 \\
.0000001 \\
.0000001 \\
.0000001 \\
.0000001 \\
.0000001 \\
.0000001 \\
.0000001 \\
.0000001 \\
.0000001\end{array}$ & $\begin{array}{l}.0000005 \\
.0000005 \\
.0000005 \\
.0000005 \\
.0000005 \\
.0000005 \\
.0000005 \\
.0000005 \\
.0000005 \\
.0000005 \\
.0000005 \\
.0000005\end{array}$ & & 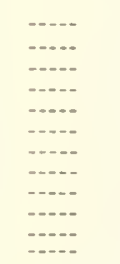 & $\begin{array}{l}.0000000 \\
.0000000 \\
.0000000 \\
.0000000 \\
.0000000 \\
.0000000 \\
.0000000 \\
.0000000 \\
.0000000 \\
.0000000 \\
.0000000 \\
.0000000\end{array}$ & & \\
\hline 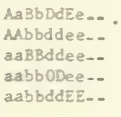 & $\begin{array}{c}.0099007 \\
\ldots \ldots \\
\cdots \ldots- \\
\ldots \ldots-\end{array}$ & $\begin{array}{l}.0012805 \\
.0000004 \\
.0000004 \\
.0000004 \\
.0000004\end{array}$ & $\begin{array}{l}.0001764 \\
.0000043 \\
.0000043 \\
.0000043 \\
.0000043\end{array}$ & & \begin{tabular}{c}
.0039840 \\
\hdashline-1 \\
$-\cdots$ \\
$-\cdots-$
\end{tabular} & $\begin{array}{l}.0005050 \\
.0000001 \\
.0000001 \\
.0000001 \\
.0000001\end{array}$ & & & \begin{tabular}{l}
.001996 \\
\hdashline$\ldots$ \\
$\ldots-$ \\
$\ldots \ldots$
\end{tabular} & & & \\
\hline $\begin{array}{l}\text { aBb Ddee-- } \\
\text { aboddEe-- } \\
\text { abbDdEe-- } \\
\text { aBbDdRe-- }\end{array}$ & $\ldots$ & $\begin{array}{l}.0012284 \\
.0012284 \\
.0012284 \\
.0012284\end{array}$ & $\begin{array}{l}.0004802 \\
.0004802 \\
.0004802 \\
.0004802\end{array}$ & $\begin{array}{l}.0001547 \\
.0001547 \\
.0001547 \\
.0001547\end{array}$ & $\begin{array}{l}-\ldots . \\
-\ldots- \\
-\ldots . \\
-\ldots-\end{array}$ & $\begin{array}{l}.0004965 \\
.0004965 \\
.0004965 \\
.0004965\end{array}$ & $\begin{array}{l}.0001894 \\
.0001894 \\
.0001894\end{array}$ & & $\begin{array}{l}\cdots-. . \\
\cdots \cdots \\
\cdots \cdots\end{array}$ & & & \\
\hline $\begin{array}{l}\text { abbddee-- } \\
\text { abbidee-- } \\
\text { LabbddEe-- } \\
\text { aabbidee- } \\
\text { aabbddEe- } \\
\text { aabbDdEe-- }\end{array}$ & $\begin{array}{l}\ldots . . . \\
\ldots \ldots \\
\ldots \ldots \\
\ldots \ldots \\
\ldots \ldots\end{array}$ & $\begin{array}{l}.0012268 \\
.0012268 \\
.0012268 \\
.0012268 \\
.0012268 \\
.0012268\end{array}$ & $\begin{array}{l}.0013866 \\
.0013866 \\
.0013866 \\
.0013866 \\
.0013866\end{array}$ & $\begin{array}{l}.0009734 \\
.0009734 \\
.0009734 \\
.0009734 \\
.0009734 \\
.0009734\end{array}$ & 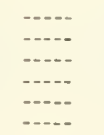 & $\begin{array}{l}.0004962 \\
.0004962 \\
.0004962 \\
.0004962 \\
.0004962 \\
.0004962\end{array}$ & & & & $\begin{array}{l}.0002491 \\
.0002491 \\
.0002491 \\
.0002491 \\
.0002491 \\
.0002491\end{array}$ & $\begin{array}{l}.0002805 \\
.0002805 \\
.0002805 \\
.0002805 \\
.0002805\end{array}$ & $\begin{array}{l}.0001921 \\
.0001921 \\
.0001921 \\
.0001921 \\
.0001921 \\
.0001921\end{array}$ \\
\hline $\begin{array}{l}\text { Aabbddee-- } \\
\text { aa Ebddee-- } \\
\text { aabbJdee. } \\
\text { aabbddRe- }\end{array}$ & 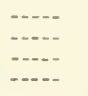 & $\begin{array}{l}.0012261 \\
.0012261 \\
.0012261 \\
.0012261\end{array}$ & $\begin{array}{l}.0040976 \\
.0040976 \\
.0040976 \\
.0040976\end{array}$ & $\begin{array}{l}.0064726 \\
.0064726 \\
.0064726 \\
.0064726\end{array}$ & ב... & $\begin{array}{l}.0004961 \\
.0004961 \\
.0004961 \\
.0004961\end{array}$ & $\begin{array}{l}.0016679 \\
.0016679 \\
.0016679\end{array}$ & $\begin{array}{r}.0026430 \\
.0026430\end{array}$ & & $\begin{array}{l}.0002490 \\
.0002490 \\
.0002490 \\
.0002490\end{array}$ & $\begin{array}{l}.0008388 \\
.0008388 \\
.0008388 \\
.0008388\end{array}$ & \\
\hline abodsee. - & .9900745 & .9814732 & .9731030 & .9675264 & .9960119 & .9925359 & .9891345 & .9868598 & .998003 & .9962590 & .9945493 & .9934045 \\
\hline
\end{tabular}


Table 33.--Relative frequencies of male genotypes when native population ( 3 autosomal genes BBDDEE plus 1 sex-1inked gene AA; female is heterogametic) is overflooded once in parental generation by $\underline{X}$ release insects ( $a$ abbddee, a-bbddee) for every native insect

\begin{tabular}{|c|c|c|c|c|c|c|c|c|c|c|c|c|}
\hline \multirow[b]{2}{*}{ Genotype } & & $\underline{x}$ & 1 & & & $x=$ & 2 & & & $\underline{x}$ & 3 & \\
\hline & $\mathrm{F}_{1}$ & $\bar{F}_{2}$ & $\mathrm{~F}_{3}$ & $\mathrm{~F}_{4}$ & $F_{1}$ & $\mathrm{~F}_{2}$ & $\mathrm{~F}_{3}$ & $\mathrm{~F}_{4}$ & $F_{1}$ & $\mathrm{~F}_{2}$ & $\mathrm{~F}_{3}$ & $\mathrm{~F}_{4}$ \\
\hline $\mathrm{AABBDDEE}_{-} 0$. & 12500000 & 0.0395508 & 0.0137806 & 0.0062786 & 0555555 & & 0.0020261 & 0.0005978 & 0.0312500 & 0.0036926 & 0.0005635 & 0.0001247 \\
\hline AABBDDEe -- & -.... & $.0087 \mathrm{~B} 91$ & $.00 B 7547$ & .0067026 & -...- & .0038580 & .0021366 & .0010855 & $\cdots$ & .0020142 & .0007745 & .0002980 \\
\hline AABBDdEE -- & -..- & .0087891 & .0087547 & .0067026 & $-\ldots$ & .0038580 & .0021366 & .0010855 & & .0020142 & .0007745 & .0002980 \\
\hline AABbDDEE -- & $-\cdots$ & .0087891 & .0087547 & .0067026 & $-\cdots$ & .0038580 & .0021366 & .0010855 & & .0020142 & .0007745 & .0002980 \\
\hline AaBBDDEE - - & ---- & .0087891 & .0087547 & .0067026 & ---- & .0038580 & .0021366 & .0010855 & $-\infty$ & .0020142 & .0007745 & .0002980 \\
\hline AABBDDee -- & $\cdots$ & $.0004 \mathrm{~B} 83$ & .0013905 & .0017889 & $\cdots$ & .0003858 & .0005633 & .0004298 & & .0002747 & .0002661 & .0001787 \\
\hline AABBddEE -- & $\ldots$ & .0004883 & .0013905 & .0017889 & $-\ldots$ & .0003858 & .0005633 & $.000429 \mathrm{~B}$ & --- & .0002747 & .0002661 & .0001787 \\
\hline AAbbDDEE -- & $-\ldots$ & $.0004 \mathrm{~B} 83$ & .0013905 & .0017889 & $-\cdots$ & .0003858 & .0005633 & .0004298 & & .0002747 & .0002661 & .0001787 \\
\hline aABBDDEE -- & ---- & .0004883 & .0013905 & .0017 BB9 & $\ldots$ & $.0003 \mathrm{~B} 58$ & .0005633 & .0004298 & & .0002747 & .0002661 & .0001787 \\
\hline AABBDdEe -- & $-\cdots$ & .0097656 & .0095901 & .0091310 & -...- & .0046296 & .0034105 & .0024213 & & .0025635 & .0015281 & $.000 \mathrm{~B} 675$ \\
\hline AABbDDEe -- & $-\cdots$ & .0097656 & .0095901 & .0091310 & $-\cdots$ & .0046296 & .0034105 & .0024213 & & .0025635 & .0015281 & .0008675 \\
\hline AABbDdEE -- & ---- & .0097656 & .0095901 & .0091310 & $\ldots$ & .0046296 & .0034105 & .0024213 & & .0025635 & :0015281 & .0008675 \\
\hline A aBBDDEe - - & $-\ldots$ & .0097656 & .0095901 & .0091310 & --- & .0046296 & .0034105 & .0024213 & & .0025635 & .0015281 & $.000 \mathrm{~B} 675$ \\
\hline AaBBDdEE -- & $-\cdots$ & .0097656 & .0095901 & .0091310 & $-\ldots$ & .0046296 & .0034105 & .0024213 & & .0025635 & .0015281 & .0008675 \\
\hline AaBb DDEE -- & $-\cdots$ & .0097656 & .0095901 & .0091310 & $-\cdots$ & .0046296 & .0034105 & .0024213 & $=--$ & .0025635 & .0015281 & .0008675 \\
\hline AABBDdee -- & -..... & .0009766 & .0021629 & .0029446 & --- & .0007716 & .0012043 & .0013036 & & .0005493 & $.0006 \mathrm{~B} 44$ & .0006119 \\
\hline AABBddEe -- & $-\ldots-$ & .0009766 & .0021629 & .0029446 & $-\ldots$ & .0007716 & .0012043 & .0013036 & & .0005493 & .0006 & .000 \\
\hline AABbDDee -- & 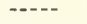 & .0009766 & .00216 .29 & .0029446 & $-\ldots-$ & .0007716 & .0012043 & .0013036 & ---- & .0005493 & .0006844 & .0006119 \\
\hline AABbddEE -- & $-\cdots$ & .0009766 & .0021629 & .0029446 & --- & .0007716 & .0012043 & .0013036 & & .0005493 & .0006844 & .0006119 \\
\hline AAbbDDEe -- & $-\cdots$ & .0009766 & .0021629 & .0029446 & $\cdots$ & .0007716 & .0012043 & .0013036 & & .0005493 & .0006844 & .0006119 \\
\hline AAbbDdEE -- & $-\cdots$ & .0009766 & .0021629 & .0029446 & -..... & .0007716 & .0012043 & .0013036 & -... & .0005493 & .0006844 & .0006119 \\
\hline A aBBDDee -- & -.... & .0009766 & .0021629 & .0029446 & --- & .0007716 & .0012043 & .0013036 & & .0005493 & .0006 & .0006119 \\
\hline AaBBddEE -- & -.... & .0009766 & .0021629 & .0029446 & $-\ldots$ & .0007716 & .0012043 & .0013036 & & .0005493 & .0006844 & .0006119 \\
\hline AabbDDEE -- & $\ldots$ & .0009766 & .0021629 & .0029446 & -.... & .0007716 & .0012043 & .0013036 & & .0005493 & .0006844 & .0006119 \\
\hline a aBBDDEE -- & $\cdots$ & .0009766 & .0021629 & .0029446 &.-- & .0007716 & .0012043 & .0013036 & & .0005493 & .0006844 & .000 \\
\hline a aBBDdEE -- & $-\cdots$ & .0009766 & .0021629 & .0029446 & --- & .0007716 & .0012043 & .0013036 & -.... & .0005493 & .0006844 & .0006119 \\
\hline a aBbDDEE -- & $\cdots$ & .0009766 & .0021629 & .0029446 & $\cdots$ & .0007716 & .0012043 & .0013036 & --- & .0005493 & .0006844 & .0006119 \\
\hline AABbDdEe -- & ---- & .0117188 & .0152435 & .0155952 & ...... & .0061728 & .0075176 & .0066767 &.--- & .0036621 & .0040078 & .0031031 \\
\hline AaBBDdEe -- & -.-- & .0117188 & .0152435 & .0155952 & ---- & .0061728 & .0075176 & .006 & & .0036621 & .0040078 & .0031031 \\
\hline AaBbDDEe-- & $\cdots$ & .0117188 & .0152435 & .0155952 & ---- & .0061728 & .0075176 & .0066767 & & .0036621 & .0040078 & .0031031 \\
\hline AaBbDdEE -- & +-- & .0117188 & .0152435 & .0155952 & ---- & .0061728 & .0075176 & .0066767 & & .0036621 & .0040078 & .0031031 \\
\hline AABBBddee -- & ---- & .0004883 & .0008411 & .0012281 & ---- & $.0003 B 58$ & .0006437 & .0008620 & & .0002747 & .0004399 & .0005237 \\
\hline AAbbDDee -- & $-\cdots$ & .0004883 & .0008411 & .0012281 & ---- & .0003858 & .0006437 & .0008620 & & .0002747 & .0004399 & .0005237 \\
\hline AAbbddEE -- & $\cdots$ & .0004883 & .0008411 & .0012281 & -... & .0003858 & .0006437 & .0008620 & & .0002747 & .0004399 & .0005237 \\
\hline aaBBDDee -- & $\cdots-$ & .0004883 & .0008411 & .0012281 & $-\cdots$ & .0003858 & .0006437 & .0008620 & & .0002747 & .0004399 & .0005237 \\
\hline aaBBddEE -- & $-\cdots$ & .0004883 & .0008411 & .0012281 & $-\cdots$ & .0003858 & .0006437 & .0008620 & & .0002747 & .0004399 & .0005237 \\
\hline aabbDDEE -- & ---- & $.00048 B 3$ & .0008411 & $.00122 \mathrm{~B} 1$ & ---- & .0003858 & .0006437 & .0008620 & & .0002747 & .0004399 & .0005237 \\
\hline AABb Ddee -- & $\cdots$ & .0019531 & .0044632 & .0060337 & -..- & .0015432 & .0033462 & .004 & & .0010986 & .0022233 & \\
\hline AABbddEe -- & 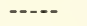 & .0019531 & .0044632 & .0060337 & -... & .0015432 & .0033462 & .00423 & & .0010986 & .0022233 & .0025 \\
\hline AAbbDdEe -- & & .0019531 & .0044632 & .0060337 & -... & .0015432 & .0033462 & .0042357 & & .0010986 & .0022233 & .0025679 \\
\hline AaBB Ddee -- & -.... & .0019531 & .0044632 & .0060337 & --- & .0015432 & .0033462 & .0042357 & & .0010986 & .0022233 & .0025679 \\
\hline AaBBddEe.-- & $\ldots-$ & .0019531 & .0044632 & .0060337 & -...- & .0015432 & .0033462 & .0042357 & & .0010986 & .0022233 & .0025679 \\
\hline AaBbDDee-- & -.... & .0019531 & .0044632 & .0060337 & $\ldots$ & .0015432 & .0033462 & .0042357 & & .0010986 & .0022233 & .0025679 \\
\hline A aBbdddEE-- & $\ldots$ & .0019531 & .0044632 & .0060337 & $-\ldots-$ & .0015432 & .0033462 & .004 & & .0010986 & .0022233 & .0025679 \\
\hline AabbDDEe-- & --- & .0019531 & .0044632 & .0060337 & - - & .0015432 & .0033462 & .0042357 & & .0010986 & .0022233 & .0025679 \\
\hline A abbDdEE - - & -...- & .0019531 & .0044632 & .0060337 &..-- & .0015432 & .0033462 & .0042357 & & .0010986 & .0022233 & .0025679 \\
\hline a aBBDdEe -- & - & .0019531 & .0044632 & .0060337 & -.... & .0015432 & .0033462 & .0042357 & & .0010986 & .0022233 & .0025679 \\
\hline a aBbDDEe-- & & .0019531 & .0044632 & .0060337 & $\cdots$ & .0015432 & .0033462 & .0042357 & & .0010986 & .0022233 & .0025679 \\
\hline aaBbDdEE-- & $\cdots$ & .0019531 & .0044632 & .0060337 & -.... & .0015432 & .0033462 & .0042357 & & .0010986 & .0022233 & .0025679 \\
\hline AABbddee-- & ---- & .0009766 & .0021629 & .0029644 & ---- & 0007716 & .0022009 & .003 & & .0005493 & .0017272 & .0026 \\
\hline AAbbDdee-- & --..- & .0009766 & .0021629 & .0029644 & -... & .0007716 & .0022009 & .0033 & & .0005493 & .0017272 & .0026030 \\
\hline AAbbddEe-- & ..... & .0009766 & .0021629 & .0029644 & --- & .0007716 & .0022009 & .003322 & & .0005493 & .0017272 & .0026030 \\
\hline AaBBddee-- & -... & .0009766 & .0021629 & .0029644 & $\cdots$ & .0007716 & .0022009 & .0033220 & & .0005493 & .0017272 & .0026030 \\
\hline AabbDDee=- & ---- & .0009766 & .0021629 & .0029644 & $-\cdots$ & .0007716 & .0022009 & .0033220 & & .0005493 & .0017272 & .0026030 \\
\hline AabbddEE -- & $-\cdots$ & .0009766 & .0021629 & .0029644 & $\cdots$ & .0007716 & .0022009 & .0033220 & --- & .0005493 & .0017272 & .0026030 \\
\hline aaBBDdee-- & ---- & .0009766 & .0021629 & .0029644 & ----- & .0007716 & .0022009 & .0033220 & & .0005493 & .0017272 & .0026030 \\
\hline aaBBddEe -- & -.... & .0009766 & .0021629 & .0029644 & $-\cdots$ & .0007716 & .0022009 & .0033220 & -... & .0005493 & .0017272 & .0026030 \\
\hline aaBbDDee-- & -- & .0009766 & .0021629 & .0029644 & --- & .0007716 & .0022009 & .0033220 & ---- & .0005493 & .0017272 & .0026030 \\
\hline aaBbddEE-- & -..-- & .0009766 & .0021629 & .0029644 & -... & .0007716 & .0022009 & .0033220 & & .0005493 & .0017272 & .0026030 \\
\hline aabbDDEe-- & $-\cdots$ & .0009766 & .0021629 & .0029644 & - . - & .0007716 & .0022009 & .0033220 & -.... & .0005493 & .0017272 & .0026030 \\
\hline a abbDdEE-- & & .0009766 & .0021629 & .0029644 & --- & .0007716 & .0022009 & .0033220 & & .0005493 & .0017272 & .0026030 \\
\hline & 2500000 & .0859375 & .0437317 & .0342350 & .2222222 & .0709876 & .0338274 & .0245779 & .1875000 & .0541992 & .0235645 & \\
\hline AAbbddee-- & -...- & .0004883 & .0013905 & .0017889 & --- & .0003858 & .0018814 & .0032005 & & & .0016952 & 2344 \\
\hline aaBBddee -- & $\ldots$ & .0004883 & .0013905 & .0017889 & -... & .0003858 & .0018814 & .0032005 & & .0002747 & .0016952 & .0032344 \\
\hline aabbDDee-- & $\cdots$ & .0004883 & .0013905 & .0017889 & $-\ldots$ & .0003858 & .0018814 & & -.... & & .0016952 & .0032344 \\
\hline aabbddEE-- & & .0004883 & .0013905 & .0017889 & $-\ldots$ & .0003858 & .0018814 & .0032005 & & .0002747 & .0016952 & .0032344 \\
\hline AaBbDdee-- & $\cdots$ & & & & $-\infty$ & & .0180610 & & -- & & & \\
\hline AaBbddEe-- & -.... & .0117188 & .0152435 & .0155950 & -.... & .0154321 & .0180610 & .0184635 & & .0153809 & 158696 & 2332 \\
\hline AabbDdEe-- & $\ldots$ & .0117188 & .0152435 & .0155950 & $-\ldots$ & .0154321 & .0180610 & & & .0153809 & .0158696 & .0152332 \\
\hline aaBbDdEe -- & $\cdots$ & .0117188 & .0152435 & .0155950 & --- & .0154321 & .0180610 & .0184635 & & .0153809 & .0158696 & .0152332 \\
\hline AaBbđdee-- & --- & .0097656 & .0095901 & .0091311 & -..- & & .016 & & -+-- & & & \\
\hline AabbDdee=- & -.... & .0097656 & & & -... & .0138889 & .0160126 & & & & & \\
\hline AabbddEe-- & --- & .0097656 & .0095901 & .0091311 & -.... & .0138889 & .0160126 & .0176403 & -.... & .0142822 & .0171321 & .0191784 \\
\hline aaBbDdee-- & -... & .0097656 & .0095901 & .0091311 & -.... & & .0160126 & & & .0142822 & & \\
\hline aaBbddEe-- & -..- & .0097656 & .0095901 & .0091311 & -... & .0138889 & .0160126 & .0176403 & & .0142822 & .0171321 & $.01917 B 4$ \\
\hline aabbDdEe-- & $-\cdots-$ & .0097656 & .0095901 & .0091311 & -... & .0138889 & .0160126 & .0176403 & & .0142822 & .0171321 & $.01917 B 4$ \\
\hline didee-- & --- & .0087891 & .0087547 & .0067027 & & .0131173 & .020 & .02165 & & .0137329 & .0269743 & .0315849 \\
\hline a aBbddee-- & -.... & .0087891 & .0087547 & .0067027 & -... & .0131173 & .0209440 & & & .0137329 & .0269743 & \\
\hline a abbDdee-- & $\cdots$ & $.0087 B 91$ & .0087547 & .0067027 & -.... & .0131173 & .0209440 & .0216570 & & .0137329 & .0269743 & .0315849 \\
\hline aabbddEe-- & $-\ldots$ & .0087891 & .0087547 & .0067027 & ---- & .0131173 & .0209440 & .0216570 & --.- & .0137329 & .0269743 & .0315849 \\
\hline Adee-- & 50000 & .0395508 & .0137806 & .0062787 & .2222222 & .1114969 & .0582889 & .0366372 & .2812500 & .1716614 & .1073034 & .0771123 \\
\hline
\end{tabular}


able 33...-ieisive frequencies of male genotypes when native population ( 3 autosoual genes BBDDEE Plus 1 sex-linked gene AA; iegal le is heterosaseticl
native insect--continued

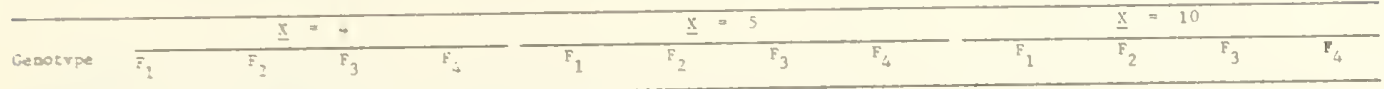

MasDDEEA, $0200000 \quad 0.0018000 \quad 0.00021840 .0000388$ ussone Nusbers 00012000.0003574 .0001113 AlasodeE- - -... $.0012000 \quad .0003576,0001118$

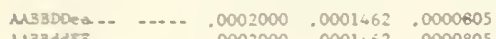

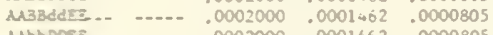
ALSDDEE_- - -... $.0002000 \quad .0001462 \quad .0000805$ aA 33 DDEE

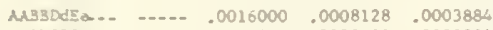

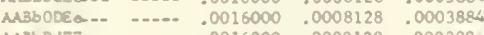

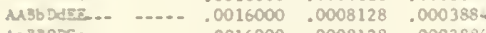

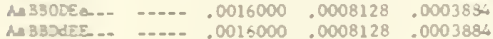
A BBDDES.- -.... $.0016000,0008128,0003884$

MBsidec-s $\quad \cdots . . .0004000 \quad .0004258 \quad .0003275$ MBBddEE--

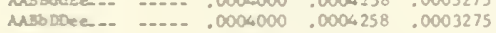
MBbddFE... …. $.0004000,0004258 \quad .0003275$ Mub DDE -. ..... .0004000,.0004258,0003275 Aubb DeIEZ-- ..... .0004000 .0004258 .0003275 MABAbDec.-. AMBSAdEE.AabbDOEE - .... .0.004000 $.0004258 \quad .0003275$

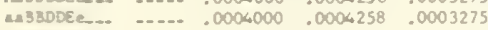
An BBDAEE.- …. $.0004000 \quad .0004258 \quad .0003275$ AMSDECE.- ..... $0004000,0004258 \quad .0003275$

MubDdEC - . ..... .0026000 .0023371 .0016693 AM 3BDdẼe...

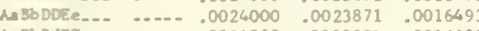

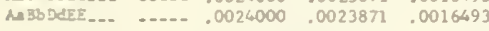

Masiddee ... ….. .0.002000,0003100,.0003331 MabbDDee .. ..... ... $0002000,0003100,0003331$ MubbddPE -.. - -.... $0002000 \quad .0003100 \quad .0003331$ LABDDEe...

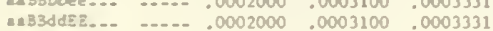
a abbDOEE... ..... .0002000,0003100,.0003331

Misb Ddee ... -..... $.0008000 \quad .0015280 \quad .0016265$ Misbd dEe -.. MbbDCEE -.. - -..- . .0008000 .0015280 .0015265 AMBBDdee -... A BBBd dEE -... ..... . .0008000 .0015280 .0016265

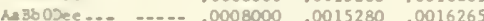
Aa Bbddes... Alabbodee...-

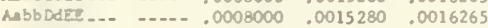
eABBDAEC... -.... $.0008000,0015280 \quad .0016265$ aA $360 D E$... LABSDCEE ... - .... $.0008000,0015280 \quad .0016265$

AMBbdtee--- $---.0004000 \quad .0013222 .0019536$ AusbDdee --. $\quad$-.... $.0004000 \quad .0013222 \quad .0019536$ MbbddE -.AnBBddee .... -..... .0004000 .0013222 .0019536 AabbDDee -.. -... 0004000.0013222 .0019536 AabbddEE … -.... $.0004000 \quad .0013222 \quad .0019536$ ea 33Dlee -.. - -... .0004000 .0013222, .0019536 aA38ddE -.. - .... . .0004000 .0013222 .0019536 a 360 Dee -.. $\begin{array}{llll}0.0019536 & .0004000 & .0013222 & .0019536\end{array}$ aabb DCEE _...

AABbDdz̄e $-.1600000 \quad .0424000 \quad .0169625 \quad .0100942$ ubbddee -..

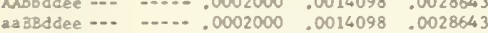
aabbodee ….... $.0002000 \quad .0014098 \quad .0028643$ aabbddEZ … -.... .0002000 $.0014098 \quad .0028643$

AabbDdee -.AaBbddEe ..- $\quad . . . . .01144000 \quad .0133520 \quad .0119863$ Aabbidze -.. - . . . . . 0144000 0133520,0119863 aABbDdze ... ...... .0144000 .0133520 .0119863

MeBbddee ... AabbDdee -.. AabbddEe -. - .... $.0136000 \quad .0164867 \quad .0181903$

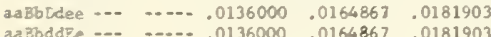
aabbDdZe -.- -... .0136000 $\quad .0164867 \quad .0181903$

Aabbdilee ... - ..... $0132000 \quad 0291466 \quad .0365424$ a \$bbddee -.. sabbDdee ... $\quad . . . .0 .0132000 \quad .0291464 \quad .0365424$ aabbdtse -..

aabbddee $-.3200000 \quad .2178000 \quad .1506456 \quad .1165559$ $\begin{array}{lllll}0.0139000 & 0.0010188 & 0.0001036 & 0.0000156\end{array}$ $\left[\begin{array}{llll}0.007837 & .0001926 & .0000512\end{array}\right.$ $\left[\begin{array}{lll}0.0007837 & .0001926 & .0000512\end{array}\right.$ ... 0007837.0001926 .0000512 $\begin{array}{llll}0.0007837 & .0001926 & .0000512\end{array}$ $\begin{array}{llll}0.0001507 & .0000895 & .0000421\end{array}$ ..... $0001507,0000895,0000421$ $\begin{array}{llll}0.0 & .0001507 & .0000895 & .0000421\end{array}$ $\ldots .0001507 .0000895 \quad .0000421$

$\left[\begin{array}{lll}0.010851 & .0004863 & .0002025\end{array}\right.$ $\begin{array}{llll}0.0010851 & .0004863 & .0002025\end{array}$ $0010851,0004863.0002025$ [.-.0010851 $0004863 \quad .0002025$ $\begin{array}{llll} & .0010851 & .0006863 & .0002025\end{array}$ $\begin{array}{lllll} & \ldots & .0010851 & .0004863 & .0002025\end{array}$

[.- . .0003014 $0002856 \quad .0001946$ $\left[\begin{array}{llll}.0003014 & .0002856 & .0001 \% 6\end{array}\right.$ .... $.0003014 \quad .0002856 \quad .0001946$ ... .0003014 .0002856 .0001946 $0003014 \quad .0002856 \quad .0001946$ $\ldots 0003014 \quad .0002856,0001946$ ... $0003014,0002856 \quad .0001946$ ... $.0003014 \quad .0002856 \quad .0001946$ ..... .0003014 .0002856 .0001946 [-. $.0003014 \quad .0002857 \quad .0001946$ $\begin{array}{llll}{[.0 .0003014} & .0002857 & .0001 \% 66\end{array}$ $\left[\begin{array}{llll}\ldots . . & .0003014 & .0002857 & .0001946\end{array}\right.$

[... $.0016879 \quad 0015492 \quad .0009740$ $\begin{array}{lll}0.0016879 & 0015492 \quad .0009740\end{array}$ [... $0016879.0015492 \quad 0009740$ $\left[\begin{array}{lll}0.0016879 & .0015492 & .00099740\end{array}\right.$ [... $\begin{array}{cccc}{[.0} & .0001507 & .0002280 & .0002248\end{array}$ $\begin{array}{llll}0 .-0 & .0001507 & .0002280 & .0002248\end{array}$ [.. $.0001507 \quad .0002280 \quad .0002248$ $\ldots 0001507 \quad 0002280 \quad 0002248$ $\ldots . .0001507,0002280.0002248$ [..- . .0006028 .0010991 .0010915 [... .0006028 .0810991 .001091 … .0006028 .0010991 .0010915 $\left[\begin{array}{llll}0.0006028 & .0010991 \quad .0010915\end{array}\right.$ [-- $.0006028 \quad .0010991 \quad .0010915$ [... .0006028 .0010991 .0010915 _...0006028 .0010991 .0010915 .... ... - 0006028.0010991 .0010915 .... .0006028 $0010991 \quad .0010915$ [... .0006028 $\quad .0010991 \quad .0010915$

[.... $.0003014 \quad .0010265 \quad .0014830$ ..... .0003014 .0010265 .0014830 ..... $.0003014 \quad .0010265 \quad .0014830$ $\ldots .0003014 .0010265 .0014830$ [... $.0003014 \quad .0010265 \quad .0014830$ $\begin{array}{llll}-0.003014 & 0010265 & 0014830\end{array}$ [.- .0003014 .0010265 .0014830 ... $0003014 \quad .0010265 \quad .0014830$ .... .0003014 .0020265 .0014830 ..... .0003014 .0010265,0014830 -... $.0003014 \quad .0010265 \quad .0014830$ --.- $.0003014 \quad .0010265 \quad .0014830$

$\begin{array}{llll}.1388888 & .0342399 & .0127442 & .0069885\end{array}$ [... $0001507 \quad .0011570 \quad .0024459$ [... .0001507 $.0011570 \quad .0026459$ $\begin{array}{llll} & 0.0001507 & .0011570 & .0024459 \\ \ldots-. .0 & .0001507 & .0011570 & .0024459\end{array}$

[... $0132620 \quad .0112640 \quad .0095031$ ......0132620 $.0112640 \quad .0095031$ [.... $0132620 \quad .0112640 \quad .0095031$ .... $0132620,0112640,0095031$ [.... $0126591 \quad 0153655,0165684$ $\left[\begin{array}{lll}0.0126591 & .0153655 & .0165684\end{array}\right.$ [... .0126591 .0153655 .0165684 $\begin{array}{llll} & 0.0 .0126591 & .0153655 & .0165684\end{array}$ $\left[\begin{array}{lll}0.0126591 & .0153655 & .0165684\end{array}\right.$ .... $0126591 \quad 0153655 \quad 0165684$ $\begin{array}{llll}0.0123577 & .0294216 & .0385180\end{array}$ $\begin{array}{lll}0.0123577 & 0294216 & 0385180\end{array}$ $\begin{array}{llll}0.0123577 & .0294216 & .0385180\end{array}$ $\left[\begin{array}{ccc}0.0123577 & .0294216 & .0385180 \\ \ldots .0385180\end{array}\right.$

$\begin{array}{llll}3472222 & .2533332 & .1870379 & .1516475\end{array}$
0.00413220 .00017290 .00001040 .0000009 $\begin{array}{llll}\ldots . . & .0001921 & .0000276 & .0000045 \\ \cdots . . & .0001921 & .0000276 & .0000045\end{array}$ $\begin{array}{llll}{[\ldots} & .0001921 & .0000276 & .0000045 \\ \ldots . .0 & .0001921 & .0000276 & .0000045\end{array}$ $\begin{array}{llll}\ldots .0 & .0001921 & .0000276 & .0000045 \\ \cdots & .0001921 & .0000276 & .0000045\end{array}$ $\begin{array}{cccc}\ldots \ldots & .0000534 & .0000183 & .0000053 \\ \cdots . .- & .0000534 & .0000183 & .0000053\end{array}$ $\begin{array}{llll}-0.0 & .0000534 & .0000183 & .0000053\end{array}$ $\begin{array}{llll}-.0 & 0000534 & .0000183 \quad 0000053\end{array}$ $\begin{array}{clll}0.0002988 & .0000925 & .0000253\end{array}$ $\begin{array}{rrrr}0.002988 & .0000925 & .0000253\end{array}$ $\begin{array}{llll}0.0002988 & .0000925 & .0000253 \\ \ldots & .0002988 & .0000925 & .0000253\end{array}$ $\begin{array}{llll}.0 .0 & .0002988 & .0000925 & .0000253 \\ & .0002988 & .0000925 & .0000253\end{array}$

$\begin{array}{llll}-0.0 & .0001067 & .0000742 & .0000349\end{array}$ $\begin{array}{llll}\cdots & .0001067 & .0000742 & .0000349\end{array}$ $\begin{array}{llll}0.0001067 & .0000742 & .0000349\end{array}$ $\begin{array}{llll}\ldots \ldots & 0001067 & .0000742 & .00000349\end{array}$ $\begin{array}{llll}{[. .} & 0001067 & 0000742 & .0000349\end{array}$ $\begin{array}{llll}\ldots .0 & .0001067 & .0000742 & .00000349 \\ \ldots .0000349\end{array}$ $\begin{array}{lllll}{[-. .0} & 0001067 & .0000742 & .0000349\end{array}$ $\begin{array}{llll}{[-.} & .0001067 & .0000742 & .0000349 \\ \cdots-. & .0001067 & .0000742 & .0000349\end{array}$ $\begin{array}{llll}\ldots . . & .0001067 & .0000742 & .0000349\end{array}$

$\ldots .0001067 .0000742,0000349$

$\begin{array}{llll}0 .- & 0005123 & .0003658 & .0001698\end{array}$ $\begin{array}{llll}\ldots .0 & .0005123 & .0003658 & .0001698\end{array}$ $\begin{array}{lllll}0.0005123 & .0003658 & .0001698\end{array}$ $\begin{array}{llll}\ldots . .0 & .0005123 & .0003658 & .0001698\end{array}$

$\begin{array}{llll}\ldots . .0 & .0000534 & .0000752 & .0000574\end{array}$ $\begin{array}{llll}\ldots .0 & .0000534 & .0000752 & .0000574\end{array}$ $\begin{array}{llll}0.0000534 & .0000752 & .0000574 \\ --.- & 0000534 & .0000752 & .0000574\end{array}$ ..... 0000534.0000752 .0000574

$\begin{array}{llll}\ldots \ldots & .0000534 & .0000752 & .0000574 \\ \ldots .0 & .0000534 & .0000752 & .0000574\end{array}$

$\left[\begin{array}{llll}0.0002134 & .0003404 & .0002699\end{array}\right.$

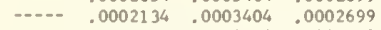

$\begin{array}{lllll} & 0.0 & .0002134 & .0003404 & .0002699\end{array}$

$\begin{array}{llll}-0.002134 & .0003404 & .0002699\end{array}$

$\begin{array}{llll}0.0 & .0002134 & .0003404 & .0002699 \\ {[-.-} & .0002134 & 0003404 & .0002699\end{array}$

..-. $.0002134 .0003404 \quad .0002699$

.... $\quad .0002134 \quad .0003404 \quad .0002699$

$\begin{array}{llll}0.0002134 & 0003404 & .0002699\end{array}$

$\begin{array}{llll}{[. .0} & .0002134 & .0003404 & .0002699\end{array}$

\begin{tabular}{llll}
{$[-0$.} & .0002134 & .0003404 & .0002699 \\
\hline-0.0002134 & .0003404 & .0002699
\end{tabular}

$\begin{array}{lllll}0 .-0 & 0001067 & .0003851 & .0005102\end{array}$

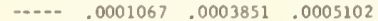
[.... $0001067 \quad 0003851 \quad .0005102$ $-0.0001067 .0003851 .0005102$ .... 0001067.0003851 .0005102 $\begin{array}{llll}{[.0} & .0001067 & .0003851 & .0005102\end{array}$ $\begin{array}{llll}0.0001067 & .0003851 & .0005102\end{array}$ $\begin{array}{llll}\ldots .- & 0001067 & .0003851 & .0005102\end{array}$ $\begin{array}{llll}0 .-0 & .0001067 & .0003851 & .0005102\end{array}$ $\begin{array}{llll}-0.0 & .001067 & .0003851 & .0005102\end{array}$ -.-. 0001067.0003851 .0005102

$\begin{array}{llll}0.0826446 & 0163069 & .0047324 & .0019635\end{array}$ [.-. 01030534.0004932 .0011345 ... $.0000534 \quad .0004932 \quad .0011345$ $\begin{array}{llll}\ldots-. & .0000534 & .0004932 & .0011345 \\ \cdots-. . & .0000534 & .0004932 & .0011345\end{array}$

[... $00089645 \quad .0058268 \quad .0039119$ $\begin{array}{llll}\ldots \ldots & .0089645 & .0058268 & .0039119\end{array}$ . . . .0089645 .0058268 .0039119 $\begin{array}{llll} & .0089645 & .0058268 & .0039119\end{array}$

[... $.0087511 .0104532 \quad .0100960$ $\begin{array}{llll}0.0 & .0087511 & .0104532 & .0100960\end{array}$ $\begin{array}{llll}-\cdots- & .0087511 & .0104532 & .0100960\end{array}$ [... $0087511 \quad .0104532 \quad .0100960$ $\left[\begin{array}{llll}-.-0 & .0087511 & .0104532 & .0100960\end{array}\right.$ [.... $.0087511 \quad 0104532 \quad .0100960$

$\begin{array}{llll}0.0086444 & 0242473 & .0348103\end{array}$

.... $\quad .0086444 \quad .0242473 \quad .0348103$

$\begin{array}{llll}\cdots-0 & .0086444 & .0242473 & .0348103 \\ \cdots-. & .0086444 & .0242473 & .0348103\end{array}$

$\begin{array}{llll}4132231 & .3500978 & .2980147 & .2670351\end{array}$ 
Table 33.--Relative frequencies of male genotypes when native population ( 3 autosoma1 genes BBOoEE plus 1 sex-1inked gene AA; female is heterogametic) is overflooded once in parental generation by $\underline{x}$ release insects (aabbddee, a-bbddee) for every native insect--Continued

\begin{tabular}{|c|c|c|c|c|c|c|c|c|c|c|c|c|}
\hline \multirow[b]{2}{*}{ Genotype } & & & $x=20$ & & & $\underline{x}$ & 50 & & & 10 & & \\
\hline & $F_{1}$ & $F_{2}$ & $F_{3}$ & $\mathrm{~F}_{4}$ & $F_{1}$ & $F_{2}$ & $F_{3}$ & $\mathrm{~F}_{4}$ & $\mathrm{~F}_{1}$ & $F_{2}$ & $\bar{F}_{3}$ & $F_{4}$ \\
\hline AABBODEE - 0 . & .0011338 & 0.0000315 & 0.0000012 & 0.0000001 & 0.0001922 & 0.0000039 & 0.00000010 & 0.0000000 & 0.0000490 & 0.0000009 & .0000000 & 0.0000000 \\
\hline AABBDOEe --- & $\ldots$ & .0000450 & .0000042 & .0000004 & --..- & .0000067 & .0000004 & .0000000 & & .0000016 & .0000001 & .0000000 \\
\hline AABBDdEE -.. & & .0000450 & .0000042 & .0000004 & & .0000067 & .0000004 & .0000000 & & .0000016 & .0000001 & .0000000 \\
\hline AABbDOEE =-- & ---- & .0000450 & .0000042 & .0000004 & $-\cdots$ & .0000067 & .0000004 & .0000000 & 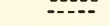 & .0000016 & .0000001 & .0000000 \\
\hline AaBBDOEE --- & 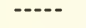 & .0000450 & .0000042 & .0000004 & $\ldots$ & .0000067 & .0000004 & .0000000 & $-\cdots$ & .0000016 & .0000001 & .0000000 \\
\hline ABBODee --- & $--\cdot$ & .0000161 & .0000038 & .0000007 & $\ldots$ & .0000029 & .0000005 & .0000001 & $\ldots$ & .0000008 & .0000001 & .0000000 \\
\hline ABBddEE --- & ---- & .0000161 & .0000038 & .0000007 & ---- & .0000029 & .0000005 & .0000001 & ---- & .0000008 & .0000001 & .0000000 \\
\hline Ab BODEE -.- & $-\cdots$ & .0000161 & .0000038 & .0000007 & $\ldots$ & .0000029 & .0000005 & .0000001 & -...- & .0000008 & .0000001 & .0000000 \\
\hline aaBBDDEE -- & $-\cdots$ & .0000161 & .0000038 & .0000007 & $\cdots$ & .0000029 & .0000005 & .0000001 & $\ldots$ & .0000008 & .0000001 & .0000000 \\
\hline AABBDdEe --- & -- & .0000771 & .0000177 & .0000033 & $-\cdots$ & .0000125 & .0000022 & .0000003 & ----- & .0000031 & .0000005 & .0000001 \\
\hline Bb00Ee -.- & -+-- & .0000771 & .0000177 & .0000033 & & .0000125 & .0000022 & .0000003 & & .0000031 & .0000005 & .0000001 \\
\hline BbOdEE & & .0000771 & .0000177 & .0000033 & $-\cdots$ & .0000125 & .0000022 & .0000003 & $-\cdots$ & .0000031 & .0000005 & .0000001 \\
\hline BBOOEe - - & $-\cdots-$ & .0000771 & .0000177 & .0000033 & --- & .0000125 & .0000022 & .0000003 & --- & .0000031 & .0000005 & .0000001 \\
\hline AaBBDdEE --- & -+- & .0000771 & .0000177 & .0000033 &..-- & .0000125 & .0000022 & .0000003 & ----- & .0000031 & .0000005 & .0000001 \\
\hline AaBbDOEE -- & $\cdots$ & .0000771 & .0000177 & .0000033 & -.... & .0000125 & .0000022 & .0000003 & -.... & .0000031 & .0000005 & .0000001 \\
\hline LBBOdee --- & -... & .0000321 & .0000180 & .0000062 & & .0000058 & .0000028 & .0000007 & & .0000015 & .0000007 & .0000002 \\
\hline BBddEe - - - & -.... & .0000 & .0000180 & .0000 & $\cdots$ & .0000058 & .0000028 & .000 & -...- & .0000015 & & \\
\hline AABb DDee -.- & $\cdots--$ & .0000321 & .0000180 & .0000062 & $\cdots$ & .0000058 & .0000028 & .0000 & $\cdots$ & .0000015 & & \\
\hline AABbddEE $=-$ & $\ldots$ & .0000321 & .0000180 & .0000062 & -...- & .0000058 & .0000028 & .0000 & -.--- & .0000015 & 107 & \\
\hline bbDDEe --- & -..... & .0000321 & .0000180 & .0000062 &.--- & .0000058 & .0000028 & .00000 & -.--- & .0000015 & .00 & .00 \\
\hline AAbbDdEE --- & $\cdots$ & .0000321 & .0000180 & .00000 & -...- & .0000058 & .0000028 & .000 & -..-- & .0000015 & & \\
\hline BBODee - -- &.--- & .0000321 & .0000180 & .0000062 & -... & .0000058 & .0000028 & .0000 & ---- & .0000015 & & \\
\hline AaBBddEE - -- & $\cdots$ & .0000321 & .0000180 & .0000062 & $-\cdots$ & .0000058 & .0000028 & .0000007 & ---- & .0000015 & .0000 & \\
\hline AabbDDEE -.- & $\cdots--$ & .0000321 & .0000180 & .000 & -...- & .0000058 & .0000028 & .000 & & .0000015 & & \\
\hline BBOOE - -. & $-\ldots$ & .0000321 & .0000180 & .0000062 & ---- & .0000058 & .0000028 & .000 & & .0000015 & .0000 & \\
\hline a aBBDdEE - & -...- & .0000321 & .0000180 & .0000062 & $\cdots$ & .0000058 & .0000028 & .0000007 & --- & .0000015 & .0000007 & .0000002 \\
\hline aABbDDEE -- & $\ldots$ & .0000321 & .0000180 & .0000062 & $-\cdots$ & .0000058 & .0000028 & .0000007 & -..- & .0000015 & .0000007 & .0000002 \\
\hline AAB DDdEe --- & & .000 & .0000822 & .000 & & .0000240 & .0000117 & .000 & & .0000 & & \\
\hline AEBBOdEe --- & & .000 & .0000822 & .000 & & .0000240 & .0000117 & .000 & & .0000061 & & \\
\hline AaBb0OEe - & --- & .0001414 & .0000822 & .0000288 & $\cdots$ & .0000240 & .0000117 & .0000031 & & .0000061 & .0000028 & .0000007 \\
\hline AaBb0dEE & $\cdots$ & .0001414 & .0000822 & .0000288 & $\cdots$ & .0000240 & .0000117 & .000 & -- & .0000061 & .0000028 & .0000 \\
\hline ddee --- & ---- & .0000 & .0000216 & .000 & $-\cdots$ & .0000029 & .0000037 & .000 & & .0000 & & \\
\hline bbDDee --- & $-\ldots$ & .000 & .0000216 & .000 & $-\ldots$ & .0000029 & .0000037 & .000 & $\ldots$ & .0000 & & \\
\hline AAbbddEE --- & --- & .0000161 & .0000216 & .0000 & --- & .0000029 & .0000037 & .0000 & & .0000008 & .0000 & .00 \\
\hline aaBBDDee -.- & $-\ldots$ & .0000161 & .0000216 & .000 & - ...- & .0000029 & .0000037 & .000 & & .0000008 & .00 & \\
\hline aEBBddEE --- & $\ldots$ & .000 & .0000216 & .0000 & -..-- & .0000029 & .0000037 & .000 & & .0000008 & .0000 & .0000005 \\
\hline \&abbODEE --- & $+\ldots$ & .0000161 & .0000216 & .0000134 & $-\ldots$ & .0000029 & .0000037 & .0000020 & & .0000008 & .0000010 & \\
\hline dee $=--$ & $-\cdots$ & .000 & .0000 & .000 & $-\ldots$ & .0000115 & .000 & .00 & & .000 & & \\
\hline AABbddEe --- & $-\cdots$ & .0000643 & .0000929 & .0000604 & --- & .0000115 & .0000155 & .000 &.-- & .0000 & 039 & \\
\hline dEe --. & $\cdots$ & .000 & .0000929 & .000 & ..... & .0000115 & .0000155 & .000 & $\ldots$ & .0000030 & .000 & 10020 \\
\hline BOdee --- & $-\ldots$ & .000 & .0000929 & .000 & -... & .0000115 & .0000155 & .000 & & .0000030 & & \\
\hline Aa BBddEe -.- & $\cdots$ & .0000643 & .0000929 & .000 & -.... & .0000115 & .0000155 & .000 & & .0000030 & 039 & 020 \\
\hline 0ee -.- & $-\ldots$ & .0000643 & .0000929 & .000 & -... & .0000115 & .0000155 & .000 & & .0000030 & & \\
\hline AaBbddEE -- - & $-\cdot-$ & .0000643 & .0000929 & .0000604 & & .0000115 & .0000155 & .0000084 & & .0000030 & .0000039 & .0000020 \\
\hline e --- & $-\cdots$ & .0000643 & .0000929 & .000 & $\ldots$ & .0000115 & .0000155 & .000 & & .0000 & .00 & 0020 \\
\hline E ... & $\ldots$ & .000 & .0000929 & .000 & & .0000115 & .0000155 & .000 & & .0000 & & \\
\hline dEe ...- & -- & .0000643 & .0000929 & .0000604 & (...... & .0000115 & .0000155 & .0000084 & -...- & .0000030 & .0000039 & .0000020 \\
\hline BbOOEe --- & $-\cdots$ & .0000643 & .0000929 & .000 & $\ldots$ & .0000115 & .0000155 & .000 & -..-- & .0000 & 039 & .000 \\
\hline aaBbOdEE --- & ---- & .0000643 & .0000929 & .0000604 & $\ldots$ & .0000115 & .0000155 & .0000084 & $-\ldots$ & .0000030 & .0000039 & \\
\hline e --- & ....- & & & & & & & & --- & .00 & & \\
\hline lee --- & $\cdots$ & & .000 & & & .0000058 & & & & .000 & & \\
\hline bddEe --- & --- & .0000321 & .0001191 & .0001458 & $-\ldots$ & .0000058 & .0000217 & .000 & -.... & .0000015 & 057 & \\
\hline Bddee _..- & -.... & & .0001191 & .00 & ..... & .0000058 & .0000217 & .000 & $-\cdots$ & .0000015 & 0057 & 0063 \\
\hline DOee _..- & $\ldots$ & & .000 & & & .0000058 & .0000217 & .000 & & .0000015 & & \\
\hline bbddEE --- & ---- & .0000321 & .0001191 & .00 & -..- & .0000058 & .0000217 & .0000248 & & .0000015 & .0000067 & 063 \\
\hline BOdee -.-- & -....- & .000 & .0001191 & & & .0000058 & & & $\ldots$ & .0000 & & \\
\hline 3BddEe --- & -- & .0000321 & .0001191 & .00 & -..- & .0000058 & .0000217 & .0000248 & & .0000015 & .0000067 & .0000063 \\
\hline Bb0Dee -.-- & --- & .000 & .0001191 & .00 & -..-- & .0000058 & .0000217 & .000 & $\ldots$ & .0000015 & 00067 & 0063 \\
\hline BbddEE $\rightarrow--$ & -..- & & & & & & & & $\ldots$ & .000 & & \\
\hline bbDOEe --- &.--- & .0000321 & .0001191 & .0001458 & -...- & .0000058 & .0000217 & .000 & & .0000015 & 067 & \\
\hline aabb DdEE --- & $\cdots+-$ & .0000321 & .0001191 & .0001458 & $-\ldots$ & .0000058 & .0000217 & .0000248 & -...- & .0000015 & .0000067 & .0000063 \\
\hline Ee--- & 0453515 & & & & 019223 & & & & 0098030 & & & \\
\hline dee--- & $-\infty-\infty$ & & & & ---- & & & & -...- & & & \\
\hline BBddee--- & $-\cdots$ & .000 & .0001643 & & --- & & .0000315 & & -.... & .0000 & & 0209 \\
\hline aabb0Dee-.- & -...- & .0000161 & .0001643 & .0003954 & $\ldots$ & .0000029 & .0000315 & .0000779 & & .0000008 & .0000084 & 0209 \\
\hline aabbddEE & & .0000161 & .0001643 & .0003954 & ---- & .0000029 & .0000315 & .0000779 & $-\ldots$ & .0000008 & .0000084 & .0000209 \\
\hline & $--\cdot-$ & & & & & & & & & & & \\
\hline Ee--- & $\cdots$ & & & & -- & & & & -...- & & & \\
\hline & ---- & .0052704 & .0027588 & .0014533 & -..-- & .0023327 & .0010199 & & -.... & .0012072 & & 1721 \\
\hline aaBbDdEQ - - & -..-- & .0052704 & .0027588 & .0014533 & $\ldots$. & .0023327 & .0010199 & .0004104 & -... & .0012072 & .0004908 & .0001721 \\
\hline & $=--$ & & & & & & & & ---- & & & \\
\hline bbDdee ...- & $\ldots$. & .0052062 & & & $-\ldots$ & & & & ----- & & & \\
\hline bbddEa..- & --- & .0052062 & .0060810 & &.--- & .0023116 & 26567 & & $-\ldots$ & .0012042 & & \\
\hline & -..- & & & & -...- & .0023116 & & .002 & - & .0012042 & .0013671 & 09877 \\
\hline & $\ldots$ & .0052062 & .0060810 & & -... & & & & ---- & & .0013671 & \\
\hline aabbDdEe -- & - & .0052062 & .0060810 & .0052035 & 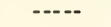 & .0023116 & .0026567 & .0020208 & $\cdots$ & .0012042 & .0013671 & .0009877 \\
\hline & ..... & & & & $-\ldots$ & & & & $--\infty$ & & & \\
\hline & & & .0158733 & & & & & & & & & \\
\hline aabb0dee -- & -.... & .0051740 & .0158733 & .0239382 & $\cdots$ & .0023154 & .0075163 & .0116881 & -... & .0012027 & .0039804 & .0062547 \\
\hline aabbddEe -- & -...- & .0051740 & .0158733 & .0239382 & -.... & .0023154 & .0075163 & .0116881 & $-\ldots$ & .0012027 & .0039804 & .0062547 \\
\hline aabbddee--- & .4535143 & .4165088 & .3832824 & .3623336 & $.4 \mathrm{~B} 05842$ & .4642351 & .4487541 & .4386308 & .4901480 & .4816920 & .4735386 & .4681402 \\
\hline
\end{tabular}


:alle 33... Reletive frequencies of mle genotypes when native population ( 3 eutosomal genes BBDDEE plus ! sex-1inked gene AA; female is heterogeticl is overflooded once in parental generation by $x$ release insects (abbddee, a-bbddee) for every native insect.--Conimied

\begin{tabular}{|c|c|c|c|c|c|c|c|c|c|c|c|c|}
\hline \multirow[b]{2}{*}{ Genseype } & & $\underline{x}=$ & 200 & & & $\underline{x}$ & 500 & & & $\underline{x}$ & 1.000 & \\
\hline & $E_{1}$ & $F_{2}$ & $\mathrm{~F}_{3}$ & $\mathrm{~F}_{4}$ & $F_{1}$ & $F_{2}$ & $\mathrm{~F}_{3}$ & $\mathrm{~F}_{4}$ & $F_{1}$ & $\mathrm{~F}_{2}$ & $F_{3}$ & $F_{L}$ \\
\hline MUSOOEE.... & $0.000012 \ldots$ & 0.00000210 & 0.0000000 & 0.0000000 & 0.0000020 & 0.0000000 & 0.0000000 & 0.0000000 & 0000005 & 0.0000000 & 0.0000000 & 0.0000000 \\
\hline AS3 3DDEe ... & .... & .0000060 & .0000000 & .0000000 & -.... & .0000001 & .0000000 & .0000000 & & .0000000 & .0000000 & .0000000 \\
\hline 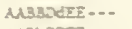 & $\ldots .$. & $.00000=0$ & .0000000 & .0000000 & $\ldots$ & .0000001 & .0000000 & .0000000 & $\ldots \ldots$ & .0000000 & .0000000 & .0000000 \\
\hline AL3B DEEE ...- & $\ldots$ & .0000040 & .0000000 & .0000000 & $\ldots .$. & .0000001 & .0000000 & .0000000 & -...- & .0000000 & .0000000 & .0000000 \\
\hline AABSDOEE -.. & $\ldots$ & $.00000 \sim 0$ & .0000000 & .0000000 & $\ldots$ & .0000001 & .0000000 & .0000000 & $\ldots-$ & .0000000 & .0000000 & .0000000 \\
\hline AHBDDEe -.. & $\ldots$ & .0000019 & .0000000 & .0000000 & $\ldots$ & .0000000 & .0000000 & .0000000 & $\ldots$ & .0000000 & .0000000 & .0000000 \\
\hline Alasddere -.. & ..... & .0000019 & .0000000 & .0000000 & $\ldots$ & .0000000 & .0000000 & .0000000 & -..... & .0000000 & .0000000 & .0000000 \\
\hline MAS B DOEE -.- & $\cdots-$ & .0000019 & .0000000 & .0000000 & .... & .0000000 & .0000000 & .0000000 & ..... & .0000000 & .0000000 & .0000000 \\
\hline asకsDDEE -.. & $\ldots$ & $.000001^{\circ}$ & .0000000 & .0000000 & $\ldots$ & .0000000 & .0000000 & .0000000 & $\ldots$ & .0000000 & .0000000 & .0000000 \\
\hline Alss zaEe -.. & $\ldots$ & $.00000^{\circ} 8$ & .0000012 & .0000000 & $\ldots$ & .0000001 & .0000000 & .0000000 & $\ldots \ldots$ & .0000000 & .0000000 & .0000000 \\
\hline MBbDISE - - - & $\ldots$. & .0000078 & .0000012 & .0000000 & $\ldots \ldots$ & .0000001 & .0000000 & .0000000 & $\ldots \ldots$ & .0000000 & .0000000 & .0000000 \\
\hline MUSDSEE -.. & $\ldots$ & .0000078 & .0000012 & .0000000 & $\ldots$ & .0000001 & .0000000 & .0000000 & -.... & .0000000 & .0000000 & .0000000 \\
\hline M 33 DoEe... & $\ldots .$. & .0000078 & .0000012 & .0000000 & -.... & .0000001 & .0000000 & .0000000 & $-\ldots$ & .0000000 & .0000000 & .0000000 \\
\hline AMBBDdER -.- & $\ldots$ & .0000078 & .0000012 & .0000000 & $\ldots$. & .0000001 & .0000000 & .0000000 & $\ldots$ & .0000000 & .0000000 & .0000000 \\
\hline AMBDDDEE-.. & $\ldots$ & .0000078 & .0000012 & .0000000 & $\ldots$ & .0000001 & .0000000 & .0000000 & $\cdots$ & .0000000 & .0000000 & .0000000 \\
\hline MHBDdee -... & ..... & .0000038 & .0000017 & .0000000 & $\ldots$ & .0000001 & .0000000 & .0000000 & $-\ldots$ & .0000000 & .0000000 & .0000000 \\
\hline MLSB ddEe - ... & ..... & .0000038 & .0000017 & .0000000 & -.... & .0000001 & .0000000 & .0000000 & $=\ldots$ & .0000000 & .0000000 & .0000000 \\
\hline MSbDDee-... & $\ldots .$. & .0000038 & .0000017 & .0000000 & $\ldots$ & .0000001 & .0000000 & .0000000 & $=\ldots$ & .0000000 & .0000000 & .0000000 \\
\hline AABbd drEE... & $\ldots$ & .0000038 & .0000017 & .0000000 & $\ldots$ & .0000001 & .0000000 & .0000 & $\ldots$ & .0000000 & .0000000 & .0000000 \\
\hline MAS DDEE.... & $\ldots .$. & .0000038 & .0000017 & .0000000 & $\ldots$. & .0000001 & .0000000 & .0000000 & 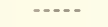 & .0000000 & .0000000 & .0000000 \\
\hline ALSDDSER -.. & $\ldots$ & .0000038 & .0000017 & .0000000 & $\ldots$ & .0000001 & .0000000 & .0000000 & $\cdots$ & .0000000 & .0000000 & .0000000 \\
\hline MABBDDee -... & $\ldots \ldots$ & .0000038 & .0000017 & .0000000 & $\ldots$ & .0000001 & .0000000 & .0000000 & $\ldots$ & .0000000 & & .000 \\
\hline MBBBd dEE -.. & ..... & .0000038 & .0000017 & .0000000 & $\ldots$ & .0000001 & .0000000 & .0000000 & $\ldots$ & .0000000 & .0000000 & .0000000 \\
\hline AAB DODEE -.- & $\ldots$ & .0000038 & .0000017 & .0000000 & $\ldots$ & .0000001 & .0000000 & .0000 & $=-\ldots$ & .0000000 & & .000 \\
\hline ABBDDCe... & $\ldots$. & .0000038 & .0000017 & .0000000 & $\ldots$ & .0000001 & .0000000 & .0000000 & 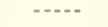 & .0000000 & .0000000 & .0000000 \\
\hline A 3BDCEE-... & $\ldots$. & .0000038 & .0000017 & .0000000 & $\ldots .$. & .0000001 & .0000000 & .0000000 & $\ldots$ & .0000000 & .0000000 & .0000000 \\
\hline A ABDDOEE- .. & $\ldots$ & .0000038 & .0000017 & .0000000 & $\ldots$ & .0000001 & .0000000 & .0000000 & $\cdots$ & .0000000 & .0000000 & .0000000 \\
\hline CẼe... & $\ldots$ & .0000015 & .0000007 & 0000001 &.--- & .0000002 & .0000001 & .0000000 & $\cdots$ & .0000001 & .0000000 & .0000000 \\
\hline M BsDdEe... & $\ldots$ & .0000015 & .0000007 & .0000001 & $\ldots$ & .0000002 & .0000001 & .0000000 & $-\cdots$ & .0000001 & 00 & .0000000 \\
\hline Aa sb Doce -... & $\ldots$ & .0000015 & .0000007 & .0000001 & & .0000002 & .0000001 & .0000000 & $\ldots$ & .0000001 & .0000000 & .0000000 \\
\hline MaBbDdrE-.. & $\ldots$ & .0000015 & .0000007 & .0000001 & $\ldots$ & .0000002 & .0000001 & .0000000 & $\ldots$ & .0000001 & .0000000 & .0000000 \\
\hline dee ... & $\ldots$ & .0000002 & .0000002 & 0000001 & $\ldots$ & .0000000 & .0000000 & .0000000 & -.... & .0000000 & .0000000 & .0000000 \\
\hline Dee.... & $\ldots$. & .0000002 & .0000002 & 0000001 & $\ldots \ldots$ & .0000000 & .0000000 & .0000000 & $\ldots$ & .0000000 & 00000 & .0000000 \\
\hline MA SddEE . .. & ..... & .0000002 & .0000002 & .0000001 & $\ldots$ & .0000000 & .0000000 & .0000000 & $\ldots$ & .0000000 & .00000 & .0000000 \\
\hline 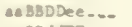 & $\ldots \ldots$ & .0000002 & .0000002 & .0000001 & $\ldots$ & .0000000 & .0000000 & .0000000 & $\cdots$ & .0000000 & .00000 & .0000000 \\
\hline A BAd BEE . ... - & -.... & .0000002 & .0000002 & .0000001 & ..... & .0000000 & .0000000 & .0000000 & ..... & .0000000 & .0000000 & .0000000 \\
\hline a $\triangle B B D D E E$... & $\ldots$ & .0000002 & .0000002 & .0000001 & $-\cdots$ & .0000000 & .0000000 & .0000000 & $\cdots$ & .0000000 & .0000000 & .0000000 \\
\hline ee.... & $\ldots .$. & .0000008 & .0000010 & 0000005 & ..... & .0000001 & .0000002 & .0000001 & $-\ldots$ & .0000000 & .0000000 & .0000000 \\
\hline MBbddEe ... & $\ldots .$. & .0000008 & .0000010 & .0000005 & $\ldots$ & .0000001 & .0000002 & .0000001 & $\ldots$ & .0000000 & .00 & 00000 \\
\hline Mb $>$ DdEe .... & ..... & .0000008 & .0000010 & .0000005 & $\ldots$ & .0000001 & .0000002 & .0000001 & $-\cdots$ & .0000000 & & 00000 \\
\hline As BBDdee ... & -.... & .00000013 & .0000010 & .0000005 & $\ldots .$. & .0000001 & .0000002 & .000 & $\ldots+$ & .0000000 & & .0000000 \\
\hline As BzddEe.... & $\ldots .$. & .0000008 & .0000010 & .0000005 & -.... & .0000001 & .0000002 & .0000001 & $\ldots$ & .0000000 & .0000000 & .0000000 \\
\hline As 36 DDee... & $\ldots .$. & .0000008 & .0000010 & .0000005 & $\ldots . .$. & .0000001 & .0000002 & .0000001 & $\ldots$ & .0000000 & .00 & .0000000 \\
\hline MabddrE... & ..... & .0000008 & .0000010 & .0000005 & $\ldots \ldots$ & .0000001 & .0000002 & .0000001 & $\ldots$ & .0000000 & 0000 & .0000000 \\
\hline MSbBDEe... & $-\ldots$ & .0000008 & .0000010 & .0000005 & $\ldots \ldots$ & .0000001 & .0000002 & .0000001 & $\ldots+$ & .0000000 & .0000000 & .0000000 \\
\hline Aabb XCEE . . - & $\ldots$ & .0000008 & .0000010 & .0000005 & $\ldots$. & .0000001 & .0000002 & .0000001 & $\ldots$ & .0000000 & .00 & .0000000 \\
\hline ABBDdEe ... & $\ldots$. & .0000008 & .0000010 & .0000005 & $\ldots$ & .0000001 & .0000002 & .0000001 & $\ldots$. & .0000000 & 00000 & .0000000 \\
\hline CABDDOEe ... & ..... & .0000008 & .0000010 & .0000005 & $\ldots$ & .0000001 & .0000002 & .0000001 & $\ldots$ & .0000000 & 00000 & .0000000 \\
\hline aABbDdEE ... & $\ldots$ & .0000008 & .0000010 & .0000005 & $\ldots$ & .0000001 & .0000002 & .0000001 & $+\cdots$ & .0000000 & .0000000 & .0000000 \\
\hline ARBbddee _ - & $\ldots .$. & .0000004 & .0000015 & .0000016 & 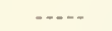 & .0000001 & .0000002 & .00 & $\ldots$ & .0000000 & .0000001 & .0000001 \\
\hline MbbDdee... & -.... & .0000004 & .0000015 & .0000016 & $\ldots$ & .0000001 & .0000002 & .00 & $\cdots$ & .0000000 & & .0000001 \\
\hline MAbbddEe ... & $-\ldots$ & .0000004 & .0000015 & .0000016 & $\ldots$. & .0000001 & .0000002 & .000 & $-\ldots$ & .0000000 & & .0000001 \\
\hline dee... & $\ldots-\ldots$ & .0000004 & .0000015 & .0000016 & ..... & .0000001 & .0000002 & .00 & $+\ldots$ & .0000000 & & .0000001 \\
\hline ASBSDDEe.... & $\ldots$ & .0000004 & .0000015 & .0000016 & $\ldots$ & .0000001 & .0000002 & .00000 & $\ldots$ & .0000000 & 00001 & .0000001 \\
\hline MAbbddEE. ... & ..... & .0000004 & .0000015 & .0000016 & ..... & .0000001 & .0000002 & & $\ldots$ & .0000000 & & 00001 \\
\hline angBDdee... & $\ldots$ & .0000604 & .0000015 & .0000016 & ..... & .0000001 & .0000002 & .00000 & $\ldots$ & .0000000 & 0001 & .0000001 \\
\hline AaBddRE ... & ..... & .0000004 & .0000015 & .0000016 & $\cdots$ & .0000001 & .0000002 & .00000 & $\ldots$ & .0000000 & .0000 & .0000001 \\
\hline a abbDDee... & ...... & .0000006 & .0000015 & .0000016 & $\ldots$ & .0000001 & .0000002 & .000000 & $\ldots$ & .0000000 & .0000001 & .0000001 \\
\hline a a 5bddFE ... & $\ldots$ & .0000004 & .0000015 & .0000016 & $\ldots$ & .0000001 & .0000002 & .0000003 & $-\ldots$ & .0000000 & 001 & .0000001 \\
\hline abbDDRe... & .... & .0000004 & .0000015 & .0000016 & $\ldots$ & .0000001 & .0000002 & .0000003 & $\cdots$ & .0000000 & & .00 \\
\hline abbDdeE ... & & .0000004 & .0000015 & .0000016 & $\ldots$ & .0000001 & .0000002 & .0000003 & $\ldots$ & .0000000 & .0000001 & .0000001 \\
\hline AA Bb DdEe $=-$ & .0049504 & .0006402 & & .000013 & 0019920 & .0002525 & .0000329 & .0006 & .0009980 & 1256 & & 0021 \\
\hline Aabbd dee -.- & -... & .0000002 & .0000022 & .0000054 & & .0000000 & .0000004 & .000 & & .0000000 & & 0002 \\
\hline Baddee -.. & $\ldots$ & .0000002 & .0000022 & .0000054 & $\ldots$ & .0000000 & .0000004 & & $+\infty$ & .0000000 & & .0000002 \\
\hline alabb DDee - & $\ldots \ldots$ & .0000002 & .0000022 & .0000054 & $\cdots$ & .0000000 & .0000004 & .0000009 & $\cdots$ & .0000000 & & .0000002 \\
\hline aabbddEE -- - & $\ldots .$. & .0000002 & .0000022 & .0000054 & $\cdots$ & .0000000 & .0000004 & .0000009 & - & .0000000 & .0000001 & .0000002 \\
\hline Aa $3 b$ D dee... & - - - & .0006141 & & & $\ldots$ & & 9947 & & & & & \\
\hline ddre-.- & $\ldots$ & .0006141 & .0002401 & .0000 & $\ldots$. & .0002483 & .0000947 & & $\ldots$ & .0001246 & & .0000140 \\
\hline AabbDdEe--- & $-\ldots$ & .0005141 & .0002401 & .0000774 & $\cdots$ & .0002483 & .0000947 & .00002 & -..... & .0001246 & 0471 & .0000140 \\
\hline aa BD Ddze-.. & & .0005141 & .0002401 & .0000774 & $\ldots$ & .0002483 & .0000947 & .0000288 & -.... & .0001246 & .0000471 & .0000140 \\
\hline La Bbddee-... & ....- & & .0006933 & .000 & $\ldots$ & & & & $\cdots$ & & & \\
\hline bDdee_.. & -...- & .0005134 & .0006933 & .0004867 & $\ldots$ & .0002481 & 02797 & & & .0001245 & 01402 & .0000960 \\
\hline MabbdIZe... & $\ldots .$. & .0006134 & .0006933 & .0004867 & $\ldots \ldots$ & .0002481 & .0002797 & .0001928 & 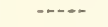 & .0001245 & 1402 & .0000960 \\
\hline 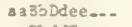 & ..... & .0006134 & .0006933 & .0004857 & $\ldots+$ & .0002481 & .0002797 & .0001928 & $\cdots+$ & .0001245 & .0001402 & .0000960 \\
\hline aa Bbddze.... & $\ldots .$. & .0006134 & .0006933 & .0004 & $-\ldots$ & .0002481 & .0002797 & .0001928 & $-\ldots$ & .0001245 & .0001402 & .0000960 \\
\hline aabbldze... & ..... & .0005134 & .0006933 & .0004867 & $\ldots$ & .0002481 & .0002797 & .0001928 & $\ldots$. & .0001245 & .0001402 & .0000960 \\
\hline bbddee-... & $\ldots$ & & & & ..... & & & & ..... & .0001245 & & \\
\hline - & -...- & .0006130 & .0020487 & .0032362 & -.... & .0002481 & .0008339 & .0013215 & $-\cdots$ & .0001245 & & .0006653 \\
\hline aabb Ddee ... - & $\ldots$. & .0005130 & .0020487 & .0032362 & $\ldots$ & .0002481 & .0008339 & .0013215 & 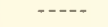 & .0001245 & .0004194 & .0006653 \\
\hline aabbddze-.- & ---- & .0006130 & .0020487 & .0032362 & $\cdots$ & .0002481 & .0008339 & .0013215 & $\cdots$ & .0001245 & .0004194 & .0006653 \\
\hline aabbddee-.- & 950372 & .4907362 & .4865513 & .4837630 & .4980059 & .4962679 & .4945670 & .4934298 & .4990014 & .4981294 & .4972746 & .4967018 \\
\hline
\end{tabular}


Table 34.--Relative frequencies of female genotypes when native population ( 3 autosomal genes BBDDEE plus 1 sex-1inked gene AA; female is heterogametic) is overflooded once in parental generation by $X$ release insects (aabbddee, a-bbddee) for every native insect

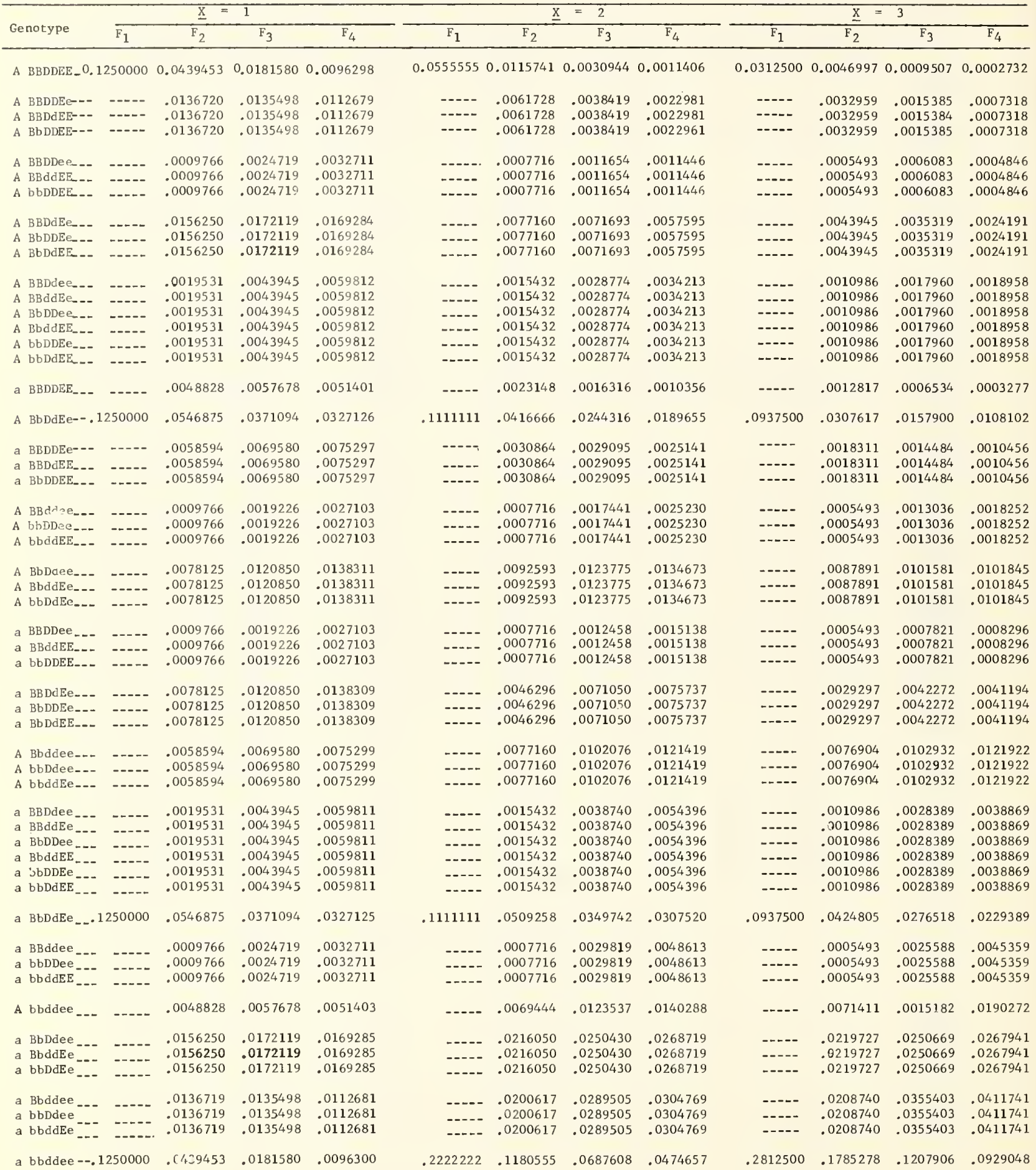


Table $3 \rightarrow .--$ Relative Erequeacies of female genotypes when native population ( 3 autosomal genes BBDDEE plus 1 sex-linked gene AA; fenale is heterogametic) is overflooded once in parental generation by $X$ release insects (aabbddee, a-bbddee) for every native insect--Continued

\begin{tabular}{|c|c|c|c|c|c|c|c|c|c|c|c|c|c|c|}
\hline \multirow{2}{*}{\multicolumn{2}{|c|}{ ienorype }} & \multicolumn{5}{|c|}{$\underline{X}=\dot{-}$} & \multicolumn{4}{|c|}{$\underline{x}=5$} & \multicolumn{4}{|c|}{$\underline{x}=10$} \\
\hline & & & $\Sigma_{1}$ & $\mathrm{~F}_{2}$ & $\mathrm{~F}_{3}$ & $\mathrm{~F}_{4}$ & $F_{1}$ & $\mathrm{~F}_{2}$ & $\mathrm{~F}_{3}$ & $F_{4}$ & $F_{1}$ & $F_{2}$ & $\mathrm{~F}_{3}$ & $\mathrm{~F}_{4}$ \\
\hline A & BBDDEE & $=0.0 ?$ & 00000 & 0.0024000 & 0.0003971 & 0.0000947 & 0.0138889 & 0.0014106 & 0.0001999 & 0.0000412 & 0.0041322 & 0.0002689 & 0.0000242 & 0.0000032 \\
\hline A & BBगDEe &.- & -.... & .002000 & .0007635 & .0003060 & -..- & .0013262 & .0004358 & .0001525 & $\cdots$ & .0003415 & .0000739 & .0000171 \\
\hline A & SBDCEE & $\ldots$ & ..... & .002000 & .0007038 & .0003060 & -.... & .0013262 & $.000 \backsim 358$ & .0001525 & ..... & .0003415 & .0000739 & .0000171 \\
\hline A & BSDDEE & ... & $\cdots$ & .002000 & .0007638 & .0003060 & $\ldots$ & $.0013=62$ & .0004358 & .0001525 & $\ldots$. & .0003415 & .0000739 & .0000171 \\
\hline A & BBDDee & -- & -... & .0006000 & .0003591 & $.000 \geq 4+2$ & $-\cdots$ & .0003014 & .0002323 & .0001394 & $\cdots$ & .0001067 & .0000554 & .0000228 \\
\hline$A$ & BBdCEE & -.. & ..... & .0000000 & .0003591 & $.0002 \omega 2$ & -.... & .0003014 & .0002323 & .0001394 & $--\cdot-$ & .0001067 & .0000554 & .0000228 \\
\hline$A$ & BSDDEE & $\ldots$ & ..... & $.000<000$ & .0003591 & $.0002 \sim \div 2$ & $\ldots$. & .000301 & .0002323 & .0001394 & $\cdots$ & .0001067 & .0000554 & .0000228 \\
\hline A & BBDdEe & ... & $\cdots$ & .0028000 & .0020064 & .0012131 & -... & .0019290 & .0012609 & .0006895 & - - - & .0005549 & .0002755 & .0001101 \\
\hline$A$ & BBDDEe & $\cdots$ & -.... & .0025000 & $.002006 \mathrm{~A}$ & .0012131 & ..... & .0019290 & .0012609 & .0006895 & -... & .0005549 & .0002755 & .0001101 \\
\hline A & BDDXEE & $\ldots$ & $\ldots$ & .0025000 & .002006 & .0012131 & $\ldots$ & .0019290 & .0012609 & .0006895 & $-\cdots$ & .0005549 & .0002755 & .0001101 \\
\hline$A$ & BBDdee & $\ldots$ & $\ldots .$. & .0003000 & .0011898 & .0011607 & .... & .0006028 & .0008351 & .0007403 & .... & .0002134 & .0002444 & .0001699 \\
\hline A & BBddEe & $\ldots$ & -... & .0008000 & .0011898 & .0011407 & -... & .0006028 & .0008351 & .0007403 & $\cdots$ & .0002134 & .0002444 & .0001699 \\
\hline$A$ & BbDDee & -- & $\ldots$. & .0008000 & .0011898 & .0011407 & $-\cdots$ & .0006028 & .0008351 & .0007403 & $\ldots$. & .0002134 & .0002444 & .0001699 \\
\hline A & BbddEE & -.. & - - - & .0003000 & .0011898 & .0011607 & - - - & .0006028 & .0008351 & .0007403 & --.. & .0002134 & .0002444 & .0001699 \\
\hline A & BODDER & $\cdots$ & $\ldots$ & .0008000 & .0011893 & $.0011 \div 07$ & $\cdots$ & .0006028 & .0008351 & .0007403 & $\cdots-$ & .0002 .134 & .0002444 & .0001699 \\
\hline A & BODDEE &.- & $\ldots$ & .0008000 & .0011898 & .0011607 & $\ldots$ & .0006028 & .0008351 & .0007403 & $\cdots$ & .0002134 & .0002444 & .0001699 \\
\hline a & BBDDEE & $\ldots$ & $\ldots$ & .0008000 & .0003249 & .0001364 & $\ldots$ & .0005425 & .0001859 & .0000677 & -.... & .0001494 & .0000321 & .0000075 \\
\hline A & BbDdẼe & -.080 & 100000 & .0235000 & .0105687 & .0066966 & .0594644 & .0188078 & .0079216 & .0046681 & .0413223 & .0086657 & .0027321 & .0011515 \\
\hline a & BBDDEe & -.. & $\cdots$ & .0012000 & .0008322 & .0005217 & -.... & .0008439 & .0005287 & .0002959 & $\ldots$ & .0002561 & .0001025 & .0000475 \\
\hline a & BB̃DdEE & $\ldots$ & ..... & .0012000 & .0008322 & .000521 & -...- & .0008439 & .0005287 & .0002959 & -.... & .0002561 & .0001025 & .0000475 \\
\hline a & $B G D D E E$ & $\ldots$ & -.... & .0012000 & .0008322 & .0005217 & $-\ldots$ & .0008439 & .0005287 & .0002959 & $\cdots$ & .0002561 & .0001025 & .0000475 \\
\hline A & BBddee & ... & 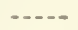 & .0004000 & .0009711 & .0013099 & ..... & .0003014 & .0007410 & .0009663 & $\ldots$ & .0001067 & .0002677 & .0003124 \\
\hline A & bb DDee & --- & -...- & .0004000 & .0009711 & .0013099 & --.- & .0003014 & .0007410 & .0009663 & -... & .0001067 & .0002677 & .0003124 \\
\hline A & bbddEE & -- & -... & .0004000 & .0009711 & .0013099 & -..- & .0003014 & .0007410 & .0009663 & $\cdots$ & .0001067 & .0002677 & .0003124 \\
\hline A & BbDdee & --- & -... & .0080000 & .0082042 & .0076196 & -...- & .0072338 & .0067313 & .0058431 & $\ldots$ & .0046957 & .0032539 & .0022260 \\
\hline A & BbddEe & -.. & $\cdots$ & .0080000 & .0082042 & .0076196 & $-\ldots$ & .0072338 & .0067313 & .005 & $-\cdots$ & .0046957 & .0032539 & .0022260 \\
\hline A & GSDdEe & $\ldots$ & $\ldots$ & .0080000 & .0082042 & .0076196 & $\ldots$ & .0072338 & .0067313 & .0058431 & $\cdots$ & .0046957 & .0032539 & .0022260 \\
\hline a & BBDDee & $\cdots$ & -.... & .0006000 & .0005229 & .0004969 & -.... & .0003014 & .0003705 & .0003221 & -... & .0001067 & .0001123 & .0000748 \\
\hline a & BBd dEE & $\cdots$ & -...- & $.000 / 4000$ & .0005229 & .0004969 & -...- & .0003014 & .0003705 & .000 & -...- & .0001067 & .0001123 & .0000748 \\
\hline a & B DDDEE & $\cdots$ & $-\ldots$ & .0004000 & .0005229 & .0006969 & 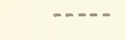 & .0003014 & .0003706 & .0003221 & $-\ldots$ & .0001067 & .0001123 & .0000748 \\
\hline a & BsDdEe & $\cdots$ & -.... & .0020000 & .0027215 & .0024511 & -.... & .0014468 & .0018737 & .0015 & -.... & .0004696 & .000 & .0003548 \\
\hline a & BbDDEe & --- & -...- & .0020000 & .0027215 & .0024511 & $\cdots$ & .0014468 & .0018737 & .0015784 & -...- & .0004696 & .0005233 & .0003548 \\
\hline a & BbDdEE & $\cdots$ & - . & .0020000 & .0027215 & .0024511 & 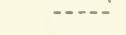 & .0014468 & .0018737 & .0015784 & $\cdots$ & .0004696 & .0005233 & .0003548 \\
\hline A & Bbddee & $\cdots$ & -... & .0072000 & .0095657 & .0110 & -.... & 66310 & .008 & & -...- & 823 & .005 & .0055583 \\
\hline A & bbDdee & $\cdots$ & -.... & .0072000 & .0095657 & .0110491 & -.... & .0066310 & .0087093 & .0097675 & $\ldots$ & .0044823 & .0056117 & .0055583 \\
\hline s & bbddEe & $\cdots$ & -.... & .0072000 & .0095657 & .0110491 & $\ldots$ & .0066310 & .0087093 & .0097675 & $\ldots$ & .0044823 & .0056117 & .0055583 \\
\hline a & BBDdee & $\cdots$ & -....- & .0008000 & .0020862 & .0027668 & $\ldots .$. & .0006028 & .0015 & .002 & $\cdots$ & .0002134 & .0005553 & .0006451 \\
\hline a & BBdCEe & -- & - & .0008000 & .0020862 & .0027668 & - & .0006028 & .0015761 & .0020 & -.... & .0002134 & .0005 & .0006451 \\
\hline a & BbD Dee & -.. & $\cdots$ & .0008000 & .0020862 & .0027668 & $-\ldots$ & .0006028 & .0015761 & .0020287 & -..-- & .0002134 & .0005553 & .0006451 \\
\hline a & BbddEE & $\cdots$ & -.... & .0008000 & .0020862 & .0027668 & -.... & .0006028 & .0015761 & .0020287 & --..- & .0002134 & .0005553 & .0006451 \\
\hline a & SWDDEe & $\cdots$ & $\cdots$ & .0008000 & .0020862 & .0027668 & - ... & .0006028 & .0015761 & .0020287 & --- & .0002134 & .0005553 & .0006451 \\
\hline a & bSDdEE & $\cdots$ & $\ldots$ & .0008000 & .0020862 & .0027668 & $\ldots$ & .0006028 & .0015761 & .0020287 & $-\cdots$ & .0002134 & .0005553 & .0006451 \\
\hline a & BbDCEe & -080 & 00000 & .0356000 & .0218332 & .0170321 & .0694444 & .0303819 & .0176361 & .0129964 & .0413223 & .0171180 & .0081931 & .0048935 \\
\hline a & BBddee & $\cdots$ & $\ldots$ & .0004000 & .0020709 & .0038411 & --- & .0003014 & .0016703 & & -.... & & & \\
\hline a & bbDDee & $\cdots$ & - & .0004000 & .0020709 & .003841 & & .0003014 & .0016703 & .0031 & & .0001067 & .0006858 & .0013895 \\
\hline a & bbddEE & $\cdots$ & -... & $.000<000$ & .0020709 & .0038411 & -.... & .0003014 & .0016703 & .0031874 & -.... & .0001067 & .0006858 & .0013895 \\
\hline A & bbddee & $\cdots$ & - - & .0068000 & .0159830 & .0211357 & $\ldots$ & .0063296 & .0158678 & .0217050 & -.... & .0043756 & .0126171 & .0185395 \\
\hline a & BbDdee & $-\cdot$ & ..... & .0208000 & .0231628 & .0241 & ..... & .0192901 & .0209976 & .02132 & $-\cdots$ & .0132334 & .0133 & .0120520 \\
\hline a & Bbddze & $\cdots$ & -..- & .0208000 & .0231628 & .0241830 & & .0192901 & .0209976 & .0213200 & -.... & .0132334 & .0133664 & .0120520 \\
\hline a & b DDdEe & $\cdots$ & $\ldots$ & .0208000 & .0231628 & .0241830 & $\ldots$ & .0192901 & .0209976 & .0213200 & $\ldots$ & .0132334 & .0133664 & .0120520 \\
\hline a & Bbddee & $\cdots$ & $\ldots$ & .0200000 & .0373897 & .045 & $-\cdots$ & .0186873 & .0371042 & .0468022 & $-\ldots$ & .0130199 & .0294741 & .0398582 \\
\hline a & & $\cdots$ & -...- & .0200000 & .0373897 & .0456376 & -.... & .0186873 & .0371042 & .0468022 & $\cdots$ & .0130199 & .0294741 & .0398582 \\
\hline a & bbdd & -.- & $\cdots$ & .0200000 & .0373897 & .0456376 & -... & .0186873 & .0371042 & .0468022 & $\cdots$ & .0130199 & .0294741 & .0398582 \\
\hline $\mathrm{a}$ & bbddee & $-=320$ & 00050 & .2243999 & .1652187 & .1348273 & 472222 & .2595120 & .2017483 & .1709067 & 132231 & .3544198 & .3101384 & .2844399 \\
\hline
\end{tabular}


Table 34.-. Relative frequencies of female genotypes when native population ( 3 autosomal genes BBDDEE plus 1 sex-1inked gene AA; female is heterogametic) is overflooded once in parental generation by $\underline{X}$ release insects (aabbddee, a-bbddee) for every native insect--Continued

\begin{tabular}{|c|c|c|c|c|c|c|c|c|c|c|c|c|}
\hline \multirow{2}{*}{ Genotype } & \multicolumn{3}{|c|}{$\underline{x}=20$} & & \multicolumn{4}{|c|}{$\underline{x}=50$} & \multicolumn{4}{|c|}{$\underline{x}=100$} \\
\hline & $\mathrm{F}_{1}$ & $\mathrm{~F}_{2}$ & $\mathrm{~F}_{3}$ & $\mathrm{~F}_{4}$ & $\overline{F_{1}}$ & $\mathrm{~F}_{2}$ & $\mathrm{~F}_{3}$ & $\overline{F_{4}}$ & $\mathrm{~F}_{1}$ & $\mathrm{~F}_{2}$ & $\overline{F_{3}}$ & $\bar{F}$ \\
\hline
\end{tabular}

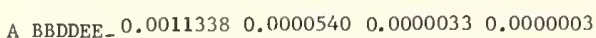

A BBDDEe -.-

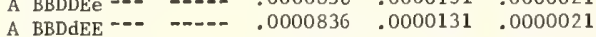

$\begin{array}{llllll}\text { A B BbDDEE -.- } & \text {-.... } & .0000836 & .00000131 & .0000021\end{array}$

$\begin{array}{llllll}\text { A BBDDee --- } & -.-- & .0000321 & .0000013 & .0000038\end{array}$

$\begin{array}{lllll}\text { A BDDDee --: } & -0 .- & .0000321 & .0000013 & .0000038\end{array}$

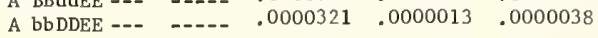

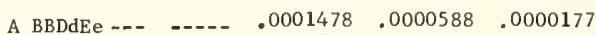

$\begin{array}{lllll}\text { A BbDDEe --. } & -.-.- & .0001478 & .0000588 & .0000177\end{array}$

A BbDdEE -.-

A BBDdEE -..

A BBddEe -..

A BbDDee -...

$\begin{array}{lllll}A & 0 \\ A & 0 & 0000643 & .0000645 & .0000364\end{array}$

A bbDDE -..

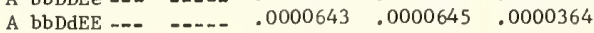

$\begin{array}{llllll}\text { a BBDDEE -. - } & \text {-.-. } & .0000386 & .0000059 & .0000009\end{array}$

$\begin{array}{llllll}\text { A BbDdEe }--.0226757 & .0038757 & .0009246 & .0002917\end{array}$

a BBDDEe --- ----- .0000707 .0000269 .0000078

a BBDdEE --- ---- $\quad .0000707 \quad 0000269 \quad .0000078$

a BbDDEE ---

A BBddee --- $\quad[--.-0000321 \quad .0000811 \quad .0000863$

$\begin{array}{lllll}\text { A B Bddee --- } & ---- & .0000321 & .0000811 & .0000863\end{array}$

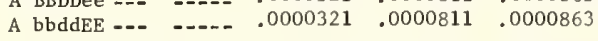

A BbDdee -..

A BbddEe -.. - -..- $.0026995 \quad .0014723 \quad .0007872$

A bbDdEe --.

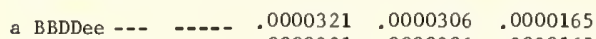

a BBddEE -..

$\begin{array}{llllll} & \text { a bbDDEE -.- } & -.-.- & .0000321 & .0000306 & .0000165\end{array}$

a BBDdEe -.. -.-.- $.0001350 \quad .0001340 \quad .0000749$

a BbDDEe -..

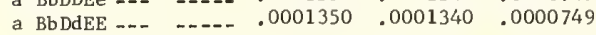

\begin{tabular}{lllll} 
A Bbddee -.. &.---- & .0026352 & .0031598 & .0027475 \\
\hline & .0026352 & .0031598 & .0027475
\end{tabular}

A bbddEe :-.

a BBDdee -.-

a BBddEe..$-1 . . .0000643 \quad .0001656 \quad .0001760$

a BbDDee ...

a BbddEE

a bbDDEe - $-0000643,0001656 \quad 0001760$

a bbDdEE

$\begin{array}{llllll}\text { a BbDdEe _.0226757 } & .0090047 & .0036011 & .0017162\end{array}$

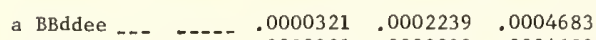

a bbDDee -.-

a bbddEE

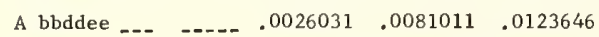

a BbDdee -.-

a BbddEe ---

a bbDdEe ...

$\begin{array}{lllll}\text { a Bbddee -- - - - } & .0077771 & .0189138 & .0265400\end{array}$

$\begin{array}{llllll}\text { a bbDdee }-- & ---- & .0077777 & .018138 & .0265400\end{array}$

$\begin{array}{llllll}\text { a bbddEe }=-- & 0 .- & 0077771 & 0189138 & .0265400\end{array}$

a bbddee--. $\begin{array}{lllll}4535145 & .4190957 & .3912190 & .3743027\end{array}$
0.00019220 .00000720 .00000030 .0000000

\begin{tabular}{|c|c|c|c|}
\hline & .0000129 & .0000015 & .0000002 \\
\hline & .0000129 & .0000015 & .0000002 \\
\hline & .0000129 & .0000015 & .0000002 \\
\hline & & .0000019 & .0000004 \\
\hline & .00 & & .00 \\
\hline & .0000058 & .000 & .000 \\
\hline & & & \\
\hline & & & \\
\hline & .0000245 & .0000081 & .0000018 \\
\hline & & & \\
\hline & .00001 & .0000105 & .0000049 \\
\hline & & & .0000049 \\
\hline & .0000115 & .00001 & .0000049 \\
\hline & & & .0000049 \\
\hline & .0000115 & .0000105 & .0000049 \\
\hline & 00000 & .0000 & \\
\hline
\end{tabular}

$\begin{array}{lllll}.0096117 & 0013872 & .0002459 & .0000550\end{array}$

---- $\quad .0000120 \quad .0000039 \quad .0000009$

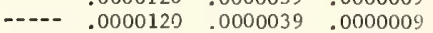

$\left[\begin{array}{llll}0 .-0 & 0000120 & .0000039 & .0000009\end{array}\right.$

[.-. $\quad .0000058 \quad .0000146 \quad .0000144$

$0.000058-0000146-.0000144$

[... $0000058 \quad .0000146 \quad .0000144$

[-.. $\quad .0011779 \quad .0005254 \quad .0002136$

[--.- $\quad .0011779 \quad .0005254 \quad .0002136$

[..- .0011779 .0005254 .0002136

$\left[\begin{array}{llll}0 .-0 & .0000058 & .0000051 & .0000023\end{array}\right.$

$\begin{array}{llll}-0 .-0 & .0000058 & .0000051 & .0000023 \\ -000058 & .0000051 & .0000023\end{array}$

[--. $.0000058 .0000051 \quad .0000023$

$\left[\begin{array}{llll} & 0000236 & .0000214 & .0000100\end{array}\right.$

[.-. $.0000236 \quad .0000214 \quad .0000100$

[... .0000236 $.0000214 \quad .0000100$

[... $0011664 \quad 0013501 \quad .0010352$

$\left[\begin{array}{llll}0.0 & 0011664 & .0013501 & .0010352\end{array}\right.$

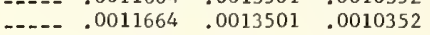

[-.

…0.000115 $.0000295 \quad .0000291$

$\left[\begin{array}{llll}0.0 & .0000115 & .0009295 & .0000291 \\ \ldots .-0 & .0000115 & .0000295 & .0000291\end{array}\right.$

[.-. .0000115 $.0000295 \quad .0000291$

$-0.000115 .000295 .000291$

[-.-. $0000115 \quad 0000295 \quad .0000291$

$\begin{array}{llll}.0096117 & 0036958 & .0012540 \quad 0004622\end{array}$

$\begin{array}{lllll} & 0000058 & .0000423 & .0000903\end{array}$

$\left[\begin{array}{cccc}0.0000058 & 0000423 & .0000903\end{array}\right.$

.-.-.

[--_.0011606 $\quad .0037896 \quad .0059220$

[... $\begin{array}{llll}0034875 & .0031667 & .0022260\end{array}$

$\begin{array}{llll}0.0034875 & .0031667 & .0022260\end{array}$

$\begin{array}{llll}\ldots .0034875 & .0031667 & .0022260\end{array}$

$\begin{array}{llll}-0.0034760 & .0088446 & .0126985\end{array}$

[.. $.0034760 \quad .0088446 \quad .0126985$

$\begin{array}{llll}0.0034760 & .0088446 & .0126985\end{array}$ $\begin{array}{lllll}0.0000490 & 0.0000017 & 0.000000 ? & 0.0000000\end{array}$

$\begin{array}{llll}-.-- & 0000032 & .0000003 & .0000000\end{array}$

$\begin{array}{lllll}---- & .0000032 & .0000003 & .0000000\end{array}$

$\begin{array}{lllll}0 .-- & .0000032 & .0000003 & .0000000\end{array}$

$\begin{array}{lllll} & 0 & 000015 & .0000005 & .0000001\end{array}$

$\begin{array}{llll}-1 .-0 & .0000015 & .0000005 & .0000001\end{array}$

$\begin{array}{llll}-.- & .000015 & .000000 & .000000001\end{array}$

$\begin{array}{lllll} & 0000062 & .0000019 & .0000004\end{array}$

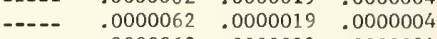

$\begin{array}{llll}{[-.-} & .0000062 & .0000019 & .00000004 \\ \cdots & .0000019 & .0000004\end{array}$

$\begin{array}{lllll}---- & .0000030 & .0000026 & .0000011\end{array}$

[-.. $.0000030 .0000026 \quad .0000011$

$\begin{array}{llll}0 .-0 & .0000030 & .0000026 & .0000011\end{array}$

.-.- $\quad .0000030 \quad .0000026 \quad .0000011$

-.-- $\quad .0000030 \quad .0000026 \quad .0000011$

[..- $\quad .0000030 \quad .0000026 \quad .0000011$

$\begin{array}{llllll}---- & .0000016 & .0000002 & .0000000\end{array}$

$\begin{array}{lllll}.0049015 & .0006609 & .0001009 & .0000183\end{array}$

$\begin{array}{lllll}0 .-0 & .0000031 & .0000009 & .0000002\end{array}$

-.-- $\quad .0000031 \quad .0000009 \quad .0000002$

[-.-. .0000031 .0000009 .0000002

$\left[\begin{array}{lllll} & 0 & 0000015 & .0000038 & .0000036\end{array}\right.$

$\begin{array}{llll}-0.0000015 & .000038 & .0000036\end{array}$

$\begin{array}{llll}0.000015 & .0000038 & .00000036\end{array}$

[--- $\quad .0006066 \quad .0002494 \quad .0000880$

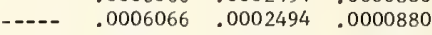

[-. $.0006066 \quad .0002494 \quad .0000880$

[--.- $\quad .0000015 \quad .0000013 \quad .0000006$

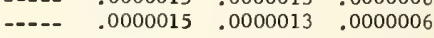

$\begin{array}{lllll}0.000015 & .0000013 & .0000006\end{array}$

$\begin{array}{llll}0 .-0 & 0000061 & .0000053 & .0000023\end{array}$

$\left[\begin{array}{llll}0 .- & .0000061 & .0000053 & .0000023\end{array}\right.$

[.-. $\quad .0000061 \quad 0000053 \quad .0000023$

[--.

$-0006036 \quad .0006892 .0005002$

$\begin{array}{llll}-.-- & .00060036 & .0006892 & .00050002\end{array}$

[-- $\quad .0000030 \quad .0000076 \quad .0000073$

[--. $.0000030 .0000076 \quad .0000073$

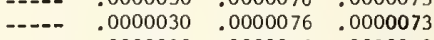

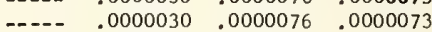

.0000030 .0000076 .0000073

-... $0000030 \quad .0000076 \quad .0000073$

$\begin{array}{lllll}.0049015 & .0018620 & .0005889 & .0001897\end{array}$

$\begin{array}{lllll}0 .-0 & .0015015 & .0000112 & .0000241\end{array}$

[.-. $0015015 \quad .0000112 .0000241$

[.-. . .0015015 .0000112,0000241

$\begin{array}{lllll} & 0018078 & 0016125 & .0010737\end{array}$

$\begin{array}{llll} & 0018078 & .0016125 & .0010737\end{array}$

$\begin{array}{llll}-.00 & .0018078 & .0016125 & .0010737\end{array}$

$\begin{array}{llll}0 .-0 & .0018078 & 0016125 & .0010737\end{array}$

$\begin{array}{lllll} & 0018048 & .0046640 & .0067485\end{array}$

...- $.0018048 \quad .0046640 \quad .0067485$

-.- $\quad .0018048 \quad .0046640 \quad .0067485$ 
Table 3-.--Relative frequencies of female genotvpes when native population ( 3 autosonal genes BBDOEE plus 1 sex-linked gene AA; feale is heterogametic) is overtlooded once in parental generation by $X$ release insects (aabbddee, a-bbddee) for every native insect--Continued

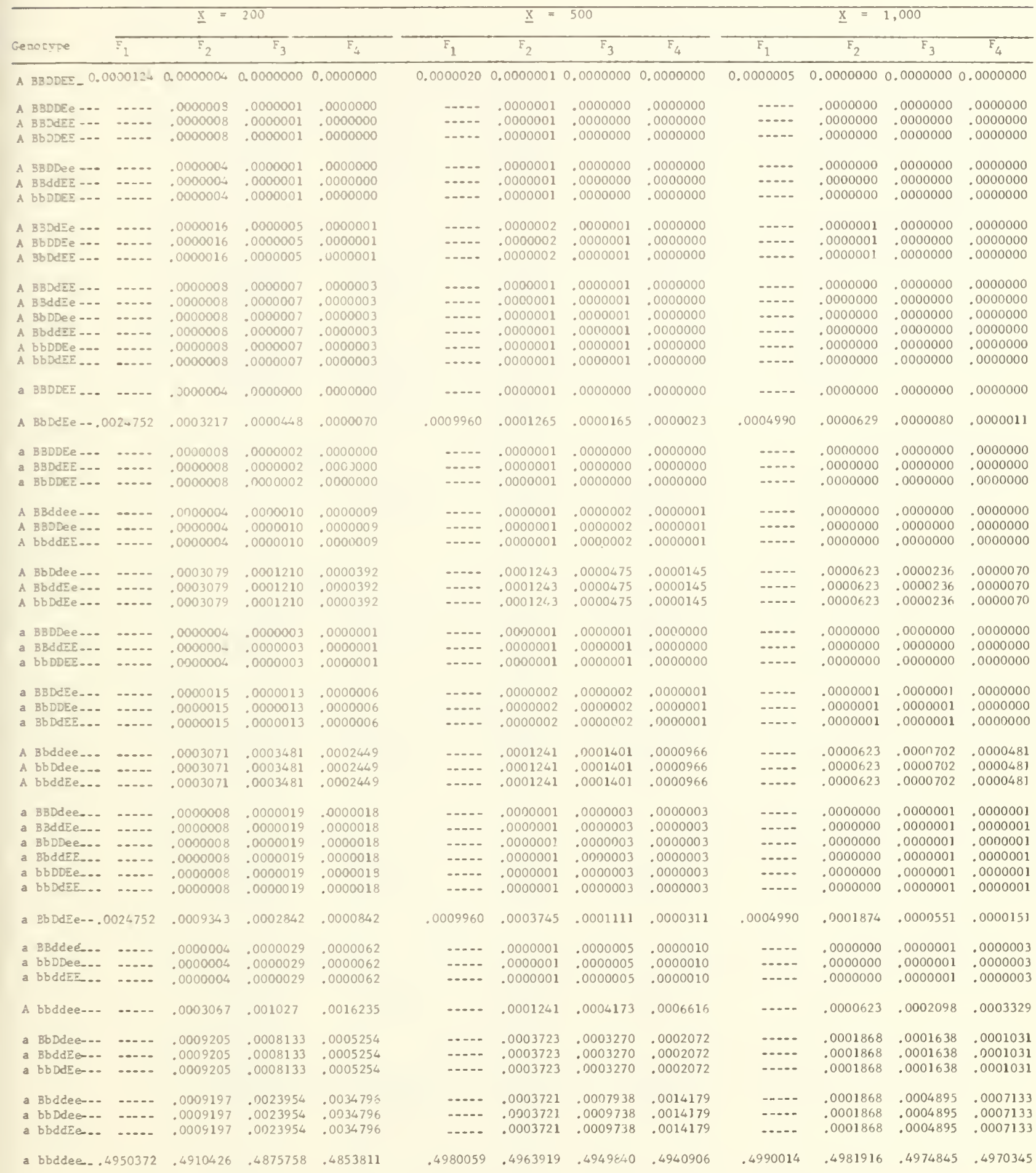


Table 35.--Relative frequencies of genotypes when native population (aa) is overflooded in parental, $F_{1}, F_{2}$, and $F_{3}$ generations by $X$ release insects for every native insect

\begin{tabular}{|c|c|c|c|c|c|}
\hline \multicolumn{2}{|c|}{ Genotype } & $\mathrm{F}_{1}$ & $\mathrm{~F}_{2}$ & $\mathrm{~F}_{3}$ & $\mathrm{~F}_{4}$ \\
\hline \multicolumn{6}{|c|}{$\underline{x}=1$} \\
\hline aa & ----- & 0.2500000 & 0.0625000 & 0.0156250 & 0.0039063 \\
\hline $\mathrm{Aa}$ & ----- & .5000000 & .3750000 & .2187500 & .1171875 \\
\hline $\mathrm{AA}$ & ---- & .2500000 & .5625000 & .7656250 & .8789063 \\
\hline \multicolumn{6}{|c|}{$\underline{x}=2$} \\
\hline aа & $\ldots--$ & .1111111 & .0204082 & .0044444 & .0010406 \\
\hline $\mathrm{Aa}$ & ---- & .4444444 & .2448981 & .1244445 & .0643498 \\
\hline $\mathrm{AA}$ & ----- & .4444444 & .7346942 & .8711112 & .9365246 \\
\hline \multicolumn{6}{|c|}{$\underline{x}=3$} \\
\hline aa & ---- & .0625000 & $.01000 \overline{0}$ & .0020661 & .0004726 \\
\hline $\mathrm{Aa}$ & ---- & .3750000 & .1800000 & .0867769 & .0425331 \\
\hline $\mathrm{AA}$ & ---- & .5625000 & .8100000 & .9111573 & .9569945 \\
\hline \multicolumn{6}{|c|}{$\underline{x}=4$} \\
\hline $\mathrm{aa}$ & ---- & .0400000 & .0059172 & .0011891 & .0002687 \\
\hline $\mathrm{Aa}$ & ---- & .3200000 & .1420119 & .0665874 & .0322494 \\
\hline $\mathrm{AA}$ & 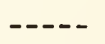 & .6400000 & .8520712 & .9322236 & .9674819 \\
\hline \multicolumn{6}{|c|}{$\underline{x}=5$} \\
\hline a a & ---- & .0277778 & .0039062 & .0007716 & .0001731 \\
\hline $\mathrm{Aa}$ & ---- & .2777777 & .1171875 & .0540123 & .0259695 \\
\hline $\mathrm{AA}$ & ----- & .6944444 & .8789064 & .9452161 & .9738574 \\
\hline \multicolumn{6}{|c|}{$\underline{x}=10$} \\
\hline aa & $-\ldots$ & .0082645 & .0010406 & .0001984 & .0000439 \\
\hline $\mathrm{Aa}$ & ---- & .1652892 & .0643495 & .0277723 & .0131573 \\
\hline $\mathrm{AA}$ & ---- & .8264462 & .9365245 & .9720310 & .9867996 \\
\hline \multicolumn{6}{|c|}{$\underline{x}=50$} \\
\hline aa & ---- & .0003845 & .0000439 & .0000081 & .0000018 \\
\hline $\mathrm{Aa}$ & $-\ldots$ & .0384467 & .0131573 & .0056818 & .0026596 \\
\hline $\mathrm{AA}$ & ----- & .9611687 & .9867991 & .9943102 & .9973388 \\
\hline \multicolumn{6}{|c|}{$\underline{x}=100$} \\
\hline aa & $-\cdots$ & .0000980 & .0000110 & .0000020 & .0000004 \\
\hline $\mathrm{Aa}$ & ---- & .0196059 & .0066224 & .0028490 & .0013316 \\
\hline $\mathrm{AA}$ & ---- & .9802959 & .9933666 & .9971490 & .9986680 \\
\hline \multicolumn{6}{|c|}{$\underline{X}=200$} \\
\hline aa & ----- & .0000248 & .0000028 & .0000005 & .0000001 \\
\hline $\mathrm{Aa}$ & ---- & .0099007 & .0033223 & .0014265 & .0006662 \\
\hline $\mathrm{AA}$ & ---- & .9900745 & .9966766 & .9985736 & .9993343 \\
\hline \multicolumn{6}{|c|}{$\underline{x}=1,000$} \\
\hline $\mathrm{aa}$ & ----- & .0000010 & .0000001 & .0000000 & .0000000 \\
\hline $\mathrm{Aa}$ & $\ldots-\ldots$ & .0019960 & .0006662 & .0002856 & .0001333 \\
\hline $\mathrm{AA}$ & 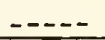 & .9980029 &, 9993339 & .9997144 & .9998668 \\
\hline
\end{tabular}


Table 30.--Relative frequencies of genotypes when native population (AABB) is overflooded in parental. $F_{1}, F_{2}$, and $F_{3}$ generations by $\underline{X}$ release insects (aabb) for every native insect

\begin{tabular}{|c|c|c|c|c|c|c|c|c|c|}
\hline $\begin{array}{c}\text { Genera- } \\
\text { tion }\end{array}$ & $\mathrm{AABB}$ & $\mathrm{AaBB}$ & $A A B b$ & $\mathrm{AaBb}$ & $\mathrm{aaBB}$ & AAbb & $\mathrm{aaBb}$ & $\mathrm{Aabb}$ & $\mathrm{a} a \mathrm{bb}$ \\
\hline & & & & $\underline{x}=1$ & & & & & \\
\hline $\begin{array}{l}F_{1}-- \\
F_{2}-\cdots \\
F_{3}-- \\
F_{4} \cdots\end{array}$ & $\begin{array}{r}0.2500000 \\
.0351563 \\
.0039063 \\
.0003815\end{array}$ & $\begin{array}{c}0 .- \\
0.0234375 \\
.0078125 \\
.0016785\end{array}$ & $\begin{array}{r}0 .- \\
0.0234375 \\
.0078125 \\
.0016785\end{array}$ & $\begin{array}{r}0.5000000 \\
.2656250 \\
.1093750 \\
.0386353 \\
\underline{X}=2\end{array}$ & $\begin{array}{r}0.0039063 \\
.0039063 \\
.0018463\end{array}$ & $\begin{array}{r}0.0039063 \\
.0039063 \\
.0018463\end{array}$ & $\begin{array}{c}0 .-- \\
0.0859375 \\
.1015625 \\
.0768738\end{array}$ & $\begin{array}{r}0.0859375 \\
.1015625 \\
.0768738\end{array}$ & $\begin{array}{r}0.2500000 \\
.4726563 \\
.6601563 \\
.8001862\end{array}$ \\
\hline $\begin{array}{l}F_{1-\ldots} \\
F_{2} \ldots- \\
F_{3} \ldots- \\
F_{4}---\end{array}$ & $\begin{array}{l}.1111111 \\
.0090703 \\
.0007281 \\
.0000578\end{array}$ & $\begin{array}{l}.0090703 \\
.0021416 \\
.0003749\end{array}$ & $\begin{array}{l}.0090703 \\
.0021416 \\
.0003749\end{array}$ & $\begin{array}{l}.4444444 \\
.1587302 \\
.0513782 \\
.0155576\end{array}$ & $\begin{array}{l}.0022676 \\
.0015747 \\
.0006078\end{array}$ & $\begin{array}{r}.0022676 \\
.0015747 \\
.0006078\end{array}$ & $\begin{array}{c}.0770975 \\
.0709247 \\
.070965025\end{array}$ & $\begin{array}{c}.0770975 \\
.0709247 \\
.0465025\end{array}$ & $\begin{array}{r}.4444444 \\
.6553288 \\
.7986117 \\
.8894141\end{array}$ \\
\hline & & & & $\underline{x}=3$ & & & & & \\
\hline $\begin{array}{l}F_{1}--- \\
F_{2}-- \\
F_{3}-- \\
E_{L^{-}---}\end{array}$ & $\begin{array}{l}.0625000 \\
.0039063 \\
.0002715 \\
.0000197\end{array}$ & $\begin{array}{l}.0046875 \\
.0009549 \\
.0001535\end{array}$ & $\begin{array}{c}-.--- \\
.0046875 \\
.0009549 \\
.0001535\end{array}$ & $\begin{array}{c}.3750000 \\
.1106250 \\
.0321810 \\
.0091212 \\
x=4\end{array}$ & $\begin{array}{l}.0014063 \\
.0008397 \\
.0002995\end{array}$ & $\begin{array}{r}.0014063 \\
.0008397 \\
.0002995\end{array}$ & $\begin{array}{c}\ldots . . \\
.0646875 \\
.0536409 \\
.0332584\end{array}$ & $\begin{array}{c}.0646875 \\
.0536409 \\
.0332584\end{array}$ & $\begin{array}{r}.5625000 \\
.7439063 \\
.8566765 \\
.9234365\end{array}$ \\
\hline $\begin{array}{l}E_{1} \ldots- \\
F_{2}--- \\
F_{3---} \\
F_{L}-\ldots\end{array}$ & $\begin{array}{l}.0400000 \\
.0021302 \\
.0001362 \\
.0000093\end{array}$ & $\begin{array}{l}.0028402 \\
.0005325 \\
.0000815\end{array}$ & $\begin{array}{l}.0028402 \\
.0005325 \\
.0000815\end{array}$ & $\begin{array}{r}.3200000 \\
.0842604 \\
.0230455 \\
.0062878 \\
X=5\end{array}$ & $\begin{array}{l}.0009467 \\
.0005204 \\
.0001779\end{array}$ & $\begin{array}{r}.0009467 \\
.0005204 \\
.0001779\end{array}$ & $\begin{array}{c}-.-- \\
.0549112 \\
.0430094 \\
.0258801\end{array}$ & $\begin{array}{l}.0549112 \\
.0430094 \\
.0258801\end{array}$ & $\begin{array}{r}.6400000 \\
.7962130 \\
.8886938 \\
.9414239\end{array}$ \\
\hline $\begin{array}{l}E_{1---} \\
F_{2---} \\
F_{3---} \\
E_{4---}\end{array}$ & $\begin{array}{l}.0277777 \\
.0013292 \\
.0000805 \\
.0000053\end{array}$ & $\begin{array}{l}.0018989 \\
.0003374 \\
.0000501\end{array}$ & $\begin{array}{c}. .-- \\
.0018989 \\
.0003374 \\
.0000501\end{array}$ & $\begin{array}{l}.2777777 \\
.0678168 \\
.0178115 \\
.0047391 \\
\underline{x}=10\end{array}$ & $\begin{array}{c}.0006782 \\
.0003537 \\
.0001177\end{array}$ & $\begin{array}{r}.00 \\
.0006782 \\
.0003537 \\
.0001177\end{array}$ & $\begin{array}{l}.0474718 \\
.0358634 \\
.0211804\end{array}$ & $\begin{array}{c}.0474718 \\
.0358634 \\
.0211804\end{array}$ & $\begin{array}{r}.6944444 \\
.8307563 \\
.9089989 \\
.9525592\end{array}$ \\
\hline $\begin{array}{l}F_{1--} \\
F_{2}-\cdots \\
F_{3} \ldots- \\
F_{4-\cdots}\end{array}$ & $\begin{array}{l}.0082645 \\
.0003096 \\
.0000166 \\
.0000010\end{array}$ & $\begin{array}{l}.0005160 \\
.0000815 \\
.0000113\end{array}$ & $\begin{array}{l}.0005160 \\
.0000815 \\
.0000113\end{array}$ & $\begin{array}{l}.1652893 \\
.0339694 \\
.0081413 \\
.0020448 \\
\underline{X}=50\end{array}$ & $\begin{array}{l}.0002150 \\
.0001003 \\
.0000316\end{array}$ & $\begin{array}{l}.0002150 \\
.0001003 \\
.0000316\end{array}$ & $\begin{array}{l}.0279495 \\
.0195494 \\
.0111012\end{array}$ & $\begin{array}{l}.0279495 \\
.0195494 \\
.0111012\end{array}$ & $\begin{array}{r}.8264463 \\
.9083600 \\
.9523796 \\
.9756660\end{array}$ \\
\hline $\begin{array}{l}F_{1} \ldots \\
E_{2} \ldots \\
F_{3} \ldots- \\
F_{4}-\cdots\end{array}$ & $\begin{array}{l}.0005845 \\
.0000114 \\
.0000005 \\
.0000000\end{array}$ & $\begin{array}{l}.0000219 \\
.0000031 \\
.0000004\end{array}$ & $\begin{array}{l}.0000219 \\
.0000031 \\
.0000004\end{array}$ & $\begin{array}{l}.0384468 \\
.0067068 \\
.0014729 \\
.0003494 \\
\underline{x}=100\end{array}$ & $\begin{array}{l}.0000105 \\
.0000045 \\
.0000013\end{array}$ & $\begin{array}{l}.0000105 \\
.0000045 \\
.0000013\end{array}$ & $\begin{array}{l}.0064286 \\
.0042057 \\
.0023097\end{array}$ & $\begin{array}{c}.--\cdots \\
.0064286 \\
.0042057 \\
.0023097\end{array}$ & $\begin{array}{r}.9611688 \\
.9803597 \\
.9900999 \\
.9950276\end{array}$ \\
\hline $\begin{array}{l}F_{1} \ldots \\
F_{2} \ldots \\
F_{3} \ldots \\
F_{4} \ldots-\end{array}$ & $\begin{array}{l}.0000980 \\
.0000028 \\
.0000001 \\
.0000000\end{array}$ & $\begin{array}{l}.0000055 \\
.0000008 \\
.0000001\end{array}$ & $\begin{array}{l}.0000055 \\
.0000008 \\
.0000001\end{array}$ & $\begin{array}{l}.0196059 \\
.0033439 \\
.0007255 \\
.0001707 \\
\underline{X}=200\end{array}$ & $\begin{array}{l}.0000027 \\
.0000011 \\
.0000003\end{array}$ & $\begin{array}{l}.0000027 \\
.0000011 \\
.0000003\end{array}$ & $\begin{array}{c}.0032730 \\
.0021227 \\
.0011607\end{array}$ & $\begin{array}{c}.--- \\
.0032730 \\
.0021227 \\
.0011607\end{array}$ & $\begin{array}{r}.9802960 \\
.9900907 \\
.9950251 \\
.9975069\end{array}$ \\
\hline $\begin{array}{l}F_{1--} \\
F_{2}--- \\
F_{3}-- \\
F_{4}=-\end{array}$ & $\begin{array}{l}.0000248 \\
.0000007 \\
.0000000 \\
.0000000\end{array}$ & $\begin{array}{l}.0000014 \\
.0000002 \\
.0000000\end{array}$ & $\begin{array}{l}.0000014 \\
.0000002 \\
.0000000\end{array}$ & $\begin{array}{l}.0099007 \\
.0016694 \\
.0003600 \\
.0000844 \\
\underline{x}=1,0\end{array}$ & $\begin{array}{l}.0000007 \\
.0000003 \\
.0000001 \\
00\end{array}$ & $\begin{array}{l}.0000007 \\
.0000003 \\
.0000001\end{array}$ & $\begin{array}{l}.0016515 \\
.0010664 \\
.0005818\end{array}$ & $\begin{array}{l}.0016515 \\
.0010664 \\
.0005818\end{array}$ & $\begin{array}{r}.9900745 \\
.9950228 \\
.9975063 \\
.9987517\end{array}$ \\
\hline $\begin{array}{l}F_{1} \ldots- \\
F_{2} \ldots- \\
F_{3}-- \\
F_{4} \cdots-\end{array}$ & $\begin{array}{l}.0000010 \\
.0000000 \\
.0000000 \\
.0000000\end{array}$ & $\begin{array}{l}.0000000 \\
.0000000 \\
.0000000\end{array}$ & $\begin{array}{l}.0000000 \\
.0000000 \\
.0000000\end{array}$ & $\begin{array}{l}.0019960 \\
.0003334 \\
.0000715 \\
.0000167\end{array}$ & $\begin{array}{l}.0000000 \\
.0000000 \\
.0000000\end{array}$ & $\begin{array}{l}.0000000 \\
.0000000 \\
.0000000\end{array}$ & $\begin{array}{l}.0003327 \\
.0002141 \\
.0001166\end{array}$ & $\begin{array}{l}.0003327 \\
.0002141 \\
.0001166\end{array}$ & $\begin{array}{r}.9980030 \\
.9990009 \\
.9995003 \\
.9997501\end{array}$ \\
\hline
\end{tabular}




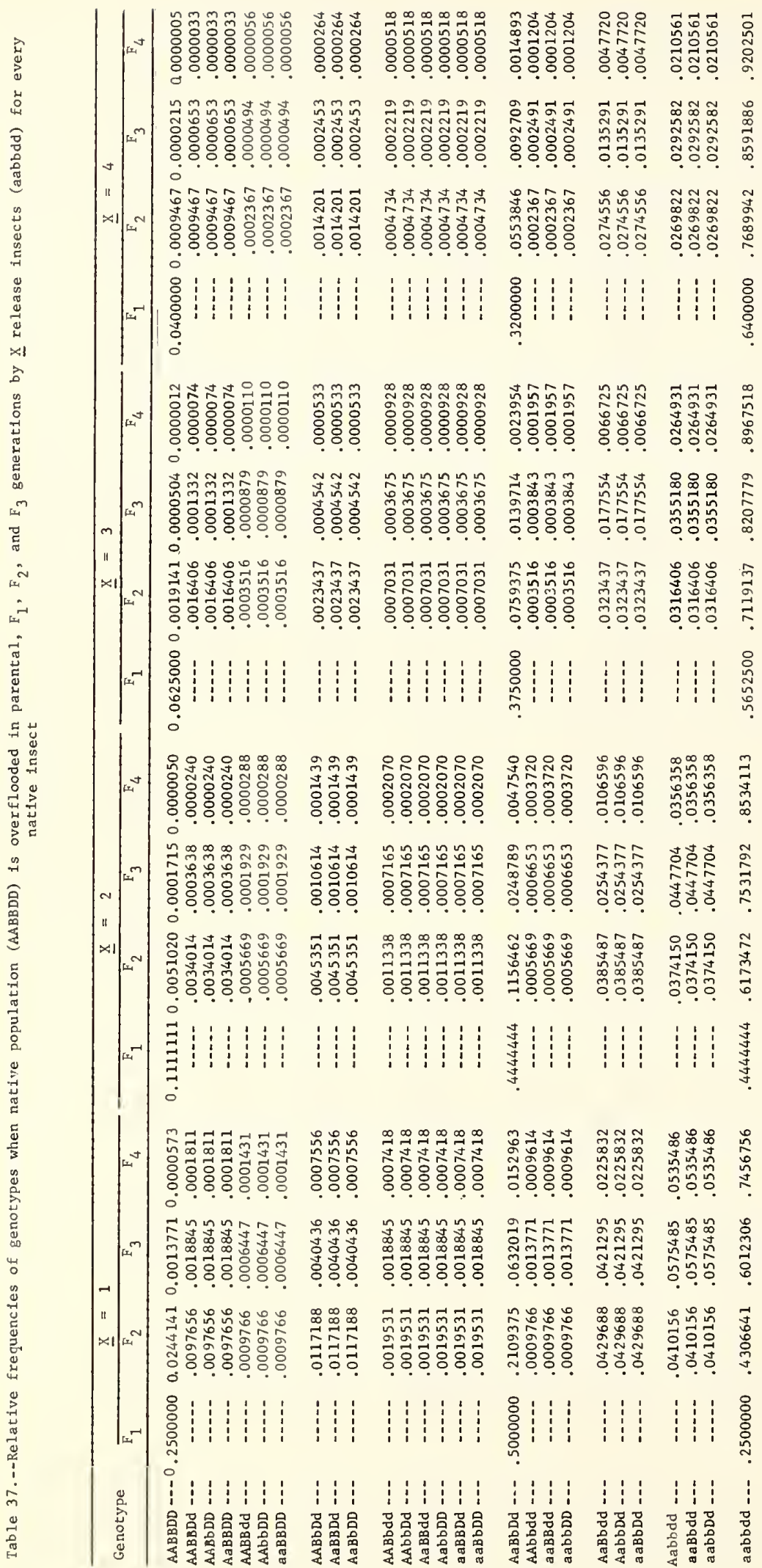




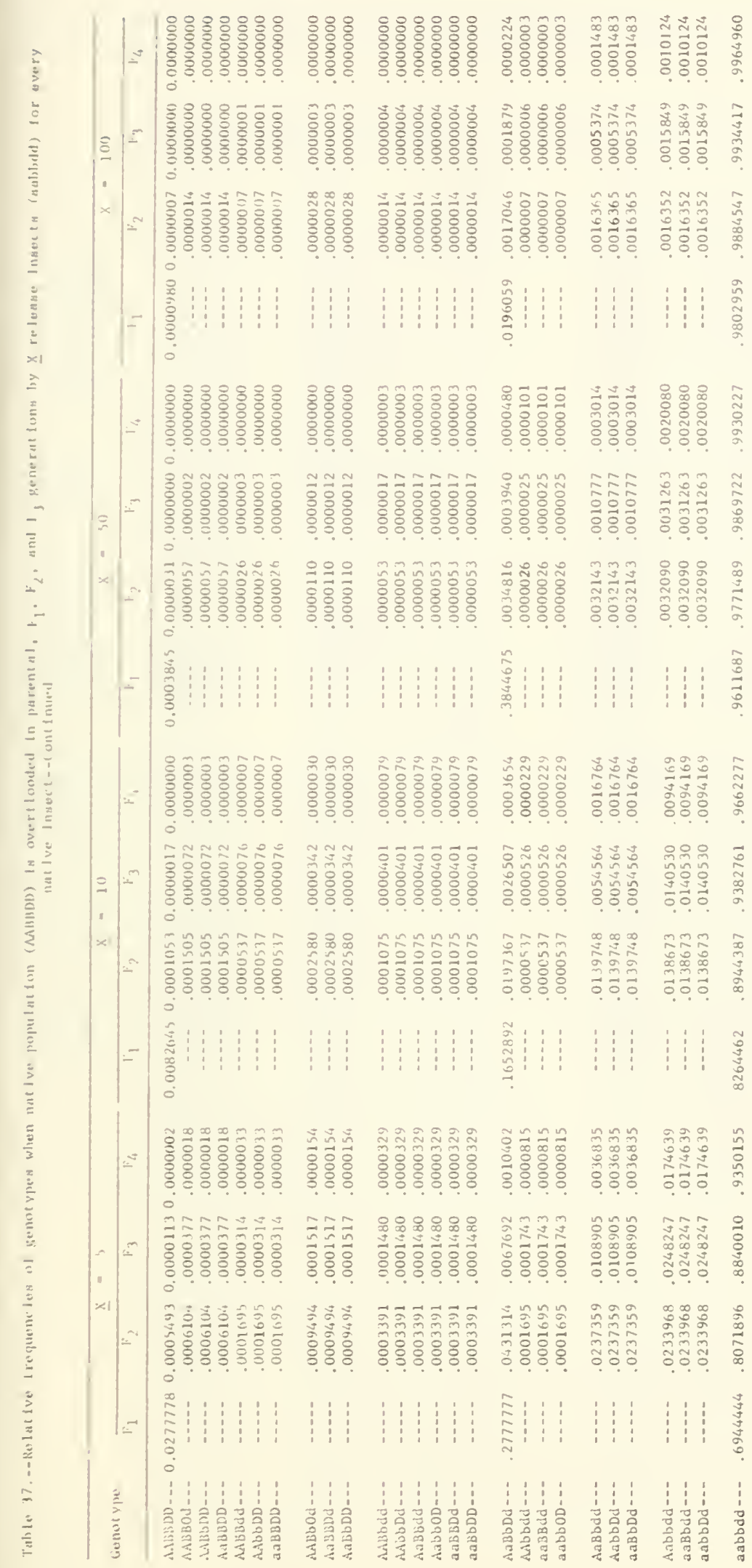


Table 37.--Relative frequencies of genotypes when native population (AABBDD) is overflooded in parenta1, $F_{1}, F_{2}$, and $F_{3}$ generations by $\underline{X}$ release insects (aabbdd) for every native insect--Continued

\begin{tabular}{|c|c|c|c|c|c|c|c|c|}
\hline \multirow[b]{2}{*}{ Genotype } & \multicolumn{4}{|c|}{$\underline{X}=200$} & \multicolumn{4}{|c|}{$\underline{X}=1,000$} \\
\hline & $\mathrm{F}_{1}$ & $\mathrm{~F}_{2}$ & $\mathrm{~F}_{3}$ & $\mathrm{~F}_{4}$ & $\mathrm{~F}_{1}$ & $\mathrm{~F}_{2}$ & $\mathrm{~F}_{3}$ & $\mathrm{~F}_{4}$ \\
\hline AABBDD - - & 0.0000248 & 0.0000002 & 0.0000000 & 0.0000000 & 0.0000010 & 0.0000001 & 0.0000000 & 0.0000000 \\
\hline AABBDd $-\ldots$ & $-\cdots$ & .0000003 & .0000000 & .0000000 & ---- & .0000001 & .0000000 & .0000000 \\
\hline AABbDD - - & ---- & .0000003 & .0000000 & .0000000 & ---- & .0000001 & .0000000 & .0000000 \\
\hline AaBBDD - - & --- & .0000003 & .0000000 & .0000000 & ---- & .0000001 & .0000000 & .0000000 \\
\hline AABBdd --- & ---- & .0000002 & .0000000 & .0000000 & ---- & .0000000 & .0000000 & .0000000 \\
\hline AAbbDD - - & ---- & .0000002 & .0000000 & .0000000 & ---- & .0000000 & .0000000 & .0000000 \\
\hline a aBBDD--- & $\cdots-$. & .0000002 & .0000000 & .0000000 & ---- & .0000000 & .0000000 & .0000000 \\
\hline $\mathrm{AABbDd}---$ & --- & .0000007 & .0000001 & .0000001 &.--- & .0000000 & .0000000 & .0000000 \\
\hline AaBBDd - - & --- & .0000007 & .0000001 & .0000001 & $-\ldots-$ & .0000000 & .0000000 & .0000000 \\
\hline $\mathrm{AaBbDD}---$ & $-\cdots-$ & .0000007 & .0000001 & .0000001 & ---- & .0000000 & .0000000 & .0000000 \\
\hline AABbdd --- & ---- & .0000003 & .0000001 & .0000000 & $-\ldots$ & .0000000 & .0000000 & .0000000 \\
\hline AAbbDd _.. & $\ldots \ldots$ & .0000003 & .0000001 & .0000000 & ---- & .0000000 & .0000000 & .0000000 \\
\hline AaBBdd ... & --- & .0000003 & .0000001 & .0000000 &.--- & .0000000 & .0000000 & .0000000 \\
\hline AabbDD__- & ---- & .0000003 & .0000001 & .0000000 & ---- & .0000000 & .0000000 & .0000000 \\
\hline a aBBDd_... & $-\ldots$ & .0000003 & .0000001 & .0000000 & $\ldots-$. & .0000000 & .0000000 & .0000000 \\
\hline a aBbDD_-- & $-\ldots-$ & .0000003 & .0000001 & .0000000 & & .0000000 & .0000000 & .0000000 \\
\hline AaBbDd_..- & .0099007 & .0008429 & .0000916 & .0000108 & .0019960 & .0001670 & .0000180 & .0000021 \\
\hline AAbbdd...- & $\ldots-$ & .0000002 & .0000002 & .0000001 & $\ldots$ & .0000001 & .0000001 & .0000000 \\
\hline aaBBdd_..- & & .0000002 & .0000002 & .0000001 & $-\ldots$ & .0000001 & .0000001 & .0000000 \\
\hline a abbDD_..- & $-\ldots$ & .0000002 & .0000002 & .0000001 & $-\ldots-$ & .0000001 & .0000001 & .0000000 \\
\hline AaBbdd_... & ---- & .0008257 & .0002683 & .0000735 & & .0001664 & .0000536 & .0000146 \\
\hline AabbDd.... & $-\ldots$ & .0008257 & .0002683 & .0000735 & & .0001664 & .0000536 & .0000146 \\
\hline a aBbDd ... & ---- & .0008257 & .0002683 & .0000735 & ---- & .0001664 & .0000536 & .0000146 \\
\hline Aabbdd...- & & .0008254 & .0007980 & .0005083 & & .0001663 & .0001605 & .0001020 \\
\hline aaBbdd... & $\ldots$ & .0008254 & .0007980 & .0005083 & & .0001663 & .0001605 & .0001020 \\
\hline a abbDd... &.--- & .0008254 & .0007980 & .0005083 & $-\ldots$ & .0001663 & .0001605 & .0001020 \\
\hline aabbdd_.-- & .9900745 & .9941983 & .9967092 & .9982441 & .9980003 & .9988348 & .9993399 & .9996479 \\
\hline
\end{tabular}


able 35..-ielative freçuencies of genotypes then native population (AABBDDEE) is overflooded in parental, $F_{1}, F_{2}$. and $F_{3}$ generations bv $x$ release insects (abbddee) for every native insect

\begin{tabular}{|c|c|c|c|c|c|c|c|c|c|c|c|c|}
\hline \multirow{2}{*}{ lesotype } & \multicolumn{4}{|c|}{$\underline{x}-1$} & & $\underline{x}=$ & $=2$ & & & $\underline{x}$ & 3 & \\
\hline & $\Sigma_{1}$ & $F_{2}$ & $E_{3}$ & $E_{4}$ & $F_{1}$ & $E_{2}$ & $F_{3}$ & $\mathrm{~F}_{4}$ & $F_{1}$ & $\mathrm{~F}_{2}$ & $\mathrm{~F}_{3}$ & $F_{4}$ \\
\hline AMS3DDED 0.25 & 500000 & 0.019775 & 0.0006323 & 0,0000120 & 0.11111110 & 0.0035431 & 0.0000554 & 0.0000006 & 0.0625000 & 0.0011816 & 0.00001310 & 0.0000001 \\
\hline $\begin{array}{l}\text { US3DDEE--. } \\
\text { MS 3DCES-.. }\end{array}$ & $\ldots-$ & .00439 .5 & .0006016 & .000028 & $\ldots$ & $\begin{array}{l}.0014172 \\
.0014172\end{array}$ & $\begin{array}{l}.0000841 \\
.0000841\end{array}$ & & $\cdots$ & $\begin{array}{l}.0006445 \\
.0006445\end{array}$ & $\begin{array}{l}.0000252 \\
.0000252\end{array}$ & $\begin{array}{l}.0000005 \\
.0000005\end{array}$ \\
\hline 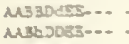 & $-\ldots$ & $.00439+5$ & .0006016 & .000025 & -...- & $\begin{array}{l}.0014172 \\
.0014172\end{array}$ & $\begin{array}{l}.0000841 \\
.0000841\end{array}$ & $\begin{array}{l}.0000023 \\
.0000023\end{array}$ & & $\begin{array}{l}.0006445 \\
.0006445\end{array}$ & .0000252 & .0000005 \\
\hline 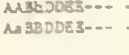 & $\cdots$ & $\begin{array}{l}.00-39-5 \\
.00-32-5\end{array}$ & $\begin{array}{l}.0006016 \\
.0006016\end{array}$ & $\begin{array}{l}.00002 S i \\
.000028\end{array}$ & -... & $\begin{array}{l}.0014172 \\
.0014172\end{array}$ & .0000841 & .0000023 & 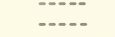 & .0006445 & .0000252 & .0000005 \\
\hline ANBSDDeC- - & & 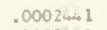 & .0001431 & .0000168 & $-\ldots$ & .0001417 & .0000320 & .0000021 & & .0000879 & .0000122 & .0000006 \\
\hline BsddBz--- & $\ldots$ & .0002401 & $.0001 \div 31$ & .0000168 & $\ldots$ & .0001417 & .0000320 & .0000021 & & .0000879 & .0000122 & .0000006 \\
\hline AADSDOES-- - & $\ldots-\infty$ & $.0002-1$ & $.0001-31$ & .0000168 & - & .0001417 & .0000320 & .0000021 & & .0000879 & .0000122 & .0000006 \\
\hline $2 \mathrm{~s}-\ldots$ & & .000241 & .0001631 & .0000168 & $-\ldots$ & .0001417 & .0000320 & .0000021 & ..... & .0000879 & .0000122 & .0000006 \\
\hline $\begin{array}{l}\text { AABBDDEC . . } \\
\text { HSB DDE }\end{array}$ & -... & .00 .8828 & .0009615 & .0000881 & $-\ldots$ & .0017007 & .0001865 & .0000104 & & .0008203 & .0000669 & .0000029 \\
\hline 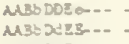 & & $.00-8828$ & .0009615 & .0000881 & $\ldots$ & .0017007 & .0001865 & .0000104 & & .0008203 & .0000669 & .0000029 \\
\hline $3 e-$. & 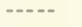 & $.00-8328$ & .0009615 & .0000881 & n.... & .0017007 & .0001865 & .0000104 & $\ldots$ & .0008203 & .0000 & .0000029 \\
\hline 38 JDEe-.- - & & $.00-5828$ & .0009615 & .0000881 & - - - & .0017007 & .0001865 & .0000104 & $-\ldots$ & .0008203 & .0000 & \\
\hline $\begin{array}{l}\text { ARSBDAZE... } \\
\text { AMBBDOEZ-.- }\end{array}$ & & $\begin{array}{l}.00 \sim 8828 \\
.00 \sim 8929\end{array}$ & $\begin{array}{l}.0009615 \\
.0009615\end{array}$ & $\begin{array}{l}.0000881 \\
.0000881\end{array}$ & $\ldots$ & .0017007 & .0001865 & .0000104 & & $\begin{array}{l}.0008203 \\
.0008203\end{array}$ & $\begin{array}{l}.0000669 \\
.0000669\end{array}$ & $\begin{array}{r}.0000029 \\
.0000029\end{array}$ \\
\hline MaBsDdee.-. & & & & & & .0017007 & .000 & .0000104 & $\ldots$ & & & .00000 \\
\hline 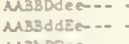 & & $\begin{array}{l}.000-833 \\
.0004883\end{array}$ & $\begin{array}{l}.0003213 \\
.0003213\end{array}$ & $\begin{array}{l}.0000645 \\
.0000645\end{array}$ & $\cdots-$ & .0002834 & .0000931 & .0000113 & & .0001758 & .0000411 & .0000039 \\
\hline 36 soec-. & - & $\begin{array}{l}.0004883 \\
.0004883\end{array}$ & $\begin{array}{l}.0003213 \\
.0003213\end{array}$ & $\begin{array}{l}.0000 \\
.00006\end{array}$ & ---- & .0002834 & .0000931 & .0000113 & & .0001758 & .0000 & \\
\hline MBbdd:3̈-.. - & $\ldots$ & $\begin{array}{l}.0004883 \\
.0006883\end{array}$ & .0003213 & $\begin{array}{l}.00006 \\
.00006\end{array}$ & 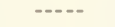 & $\begin{array}{l}.0002834 \\
.0002834\end{array}$ & .0000931 & .0000113 & $\cdots$ & 01758 & .0000 & .000 \\
\hline Must $208 \mathrm{e}--$ & $-\ldots$ & .0004883 & .0003213 & $\begin{array}{l}.0000665 \\
.0000625\end{array}$ & $\ldots$ & $\begin{array}{l}2834 \\
2834\end{array}$ & .000 & 00113 & -..- & $\begin{array}{l}01758 \\
01758\end{array}$ & .0000 & .000 \\
\hline W' DAZZ - - - & $\ldots$ & .0004883 & $.0003 \$ 13$ & $.000066^{2}$ & $-\cdots$ & $\begin{array}{l}.0002834 \\
.0002834\end{array}$ & & $\begin{array}{l}.0000 \\
.0000\end{array}$ & $\ldots+$ & $\begin{array}{l}01758 \\
01758\end{array}$ & .000 & .00 \\
\hline ASBDDea- - & $-\ldots$ & .0004883 & .0003213 & .0000645 & $\ldots$ & .0002834 & .0000931 & $\begin{array}{l}.0000113 \\
.0000113\end{array}$ & $-\ldots$ & $\begin{array}{l}758 \\
758\end{array}$ & .000 & $\begin{array}{l}.00 \\
.00\end{array}$ \\
\hline Bddzs:-- & & $.000<883$ & .0003213 & .0000645 & $\ldots$. & .0002834 & .0000931 & $\begin{array}{l}.0000113 \\
.0000113\end{array}$ & $\cdots$ & .0001758 & .0000411 & $\begin{array}{l}.0000039 \\
.0000039\end{array}$ \\
\hline DbDOEE- - - & & .0004883 & .0003213 & .0000665 & $-\ldots-$ & .0002834 & .0000931 & .0000113 & & .0001758 & .0000411 & .0000039 \\
\hline 30DEe- -. & $-\cdots$ & .0004883 & .0003213 & .0000645 & -.... & .0002834 & .0000931 & .0000113 & $\ldots .$. & .0001758 & .0000411 & .00 \\
\hline $\begin{array}{l}\triangle A B B D C E S- \\
\text { ABSDDDEE -- }\end{array}$ & & .0004883 & .0003213 & .0000645 & $-\ldots$ & .0002834 & .0000931 & .0000113 & $-\ldots$ & .0001758 & .0000411 & .0000039 \\
\hline$\triangle A B D D E$ - - & $\cdots+$ & .0004883 & .0003213 & .0000665 & $-\ldots$ & .0002834 & .0000931 & .0000113 & $-\cdots$ & .0001758 & .0000411 & .0000039 \\
\hline 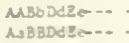 & ----- & .0058596 & .0021548 & .0003539 & $-\ldots$ & .0022676 & .0005407 & .0000584 & & .0011719 & .0002223 & .0000197 \\
\hline BaDdEe-. & $\cdots$ & .0058596 & $.00215-8$ & .0003539 & $=-.-$ & .0022676 & .0005407 & .000 & & .0011719 & .000 & \\
\hline $\begin{array}{l}\text { AMB b DDEE-- - } \\
\text { MA3SDAZ3 - - }\end{array}$ & --- & .0058594 & .0021548 & .0003539 & $-\cdots$ & .0022676 & .0005497 & .0000584 & $-\ldots$ & .0011719 & .0002223 & .00 \\
\hline Aa3s DePZ -. & $--\cdot$ & .0068594 & .0021566 & .0003539 & $=-\ldots$ & .0022676 & .0006497 & .0000584 & $\cdots$ & .0011719 & .0002223 & .0000197 \\
\hline $\begin{array}{l}\text { Mussddee-.- } \\
\text { MAbbDDee-.- }\end{array}$ & $-\infty \ldots$ & $.0002 \sin 1$ & .0001803 & .0000618 & 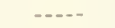 & .0001417 & .0000678 & .0000154 & & .0000879 & .0000346 & .0000064 \\
\hline $\begin{array}{l}\text { MAbbDDee-.- } \\
\text { MabdeEE -. }\end{array}$ & $-\cdots$ & .0002441 & .0001803 & .00006 & -...- & 417 & .000 & .000 & ..... & 000879 & .000 & \\
\hline $\begin{array}{l}\text { bddEE -- } \\
\text { BDDee-- }\end{array}$ & $\cdots$ & $.0002 \omega 41$ & .0001803 & .0000618 & -...- & 001417 & .0000678 & .0000154 & $\ldots$ & .0000879 & & \\
\hline BddzB -. & --- & $.0002-1$ & .0001803 & .0000168 & $\ldots$ & .0001417 & .0000678 & .0000154 & & 00879 & .0000 & \\
\hline 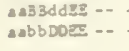 & $\cdots$ & .000241 & .0001803 & .0000168 & $-\cdots$ & 1417 & .0000678 & .0000154 & ..... & .0000879 & .0000346 & 064 \\
\hline & & $.0002<41$ & .0001803 & .000 & $-\cdots$ & .0001417 & .0000678 & .0000154 & $\ldots$ & .0000879 & .0000346 & .0000064 \\
\hline $\begin{array}{l}\text { MBbbdee -- } \\
\text { MBbddZe -- }\end{array}$ & $-\ldots$ & .0009766 & .0009272 & .0003135 & $-\cdots-$ & .0005669 & .0003342 & .0000751 & & .0003516 & .0001650 & .0000307 \\
\hline $\begin{array}{l}\text { AMBbddZE -. } \\
\text { LASBDCEe .. }\end{array}$ & & 0009766 & .0009272 & .0003135 &..-- & .0005669 & .0003342 & .000 & & & & \\
\hline $\begin{array}{l}\text { LAbbDCZe -. } \\
\text { ABBDdee - }\end{array}$ & $\ldots$ & 009766 & .0009272 & .0003135 & 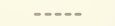 & .0005669 & .0003342 & .000 & & 0003516 & .0001 & \\
\hline $\begin{array}{l}\text { ABBDdee .- } \\
\text { MBBddZe .. }\end{array}$ & $\ldots$ & .0009766 & .0009272 & .0003135 & $-\ldots$ & .0005669 & .0003342 & .0000751 & & 03516 & .000 & \\
\hline MBBAdZe -.. & --- & .0009766 & .0009272 & .0003135 & --- & .0005669 & .0003342 & .0000751 & $\ldots$ & .0003516 & .0001 & \\
\hline $\begin{array}{l}\text { MaBbDDCe -. } \\
\text { M BbddZZ -. }\end{array}$ & ---- & .0009766 & .0009272 & .0003135 & -...- & .0005669 & .0003342 & .0000751 & -...- & .0003516 & .0001 & \\
\hline $\begin{array}{l}\text { A BbddZZ -. } \\
\text { AabbDDEe -. }\end{array}$ &.-- & 0009766 & .0009272 & .0003135 & $\ldots$ & .0005669 & .0003342 & .0000751 & $\ldots$ & 0003516 & 1650 & .0000307 \\
\hline 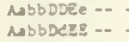 & & 0009766 & .0009272 & .0003135 & -.... & .0005669 & .0003342 & .0000751 & $\ldots$ & .0003516 & .0001 & \\
\hline $\begin{array}{l}\text { ADS } \triangle D C E Z \text {-- } \\
\triangle A B B D d Z E\end{array}$ & -- & .0009766 & .0009272 & .0003135 & $-\cdots$ & .0005669 & .0003342 & .0000751 &.-- & .0003516 & & \\
\hline $\begin{array}{l}\text { AB BBDdZe -. } \\
\text { AABSDDZe -- }\end{array}$ & $\ldots$ & 0009766 & .0009272 & .0003135 & --- & .0005669 & .0003342 & .00 & & .0003516 & .00016 & \\
\hline $\begin{array}{l}\text { AABSDDRe -- } \\
\triangle A B S D C Z Z=-\end{array}$ & $\ldots$ & .0009766 & .0009272 & .0003135 & $-\ldots$ & .0005669 & .0003342 & .000 & $\ldots$ & .0003516 & .00016 & \\
\hline$\triangle A B \supset D C Z Z--$ & 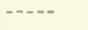 & .0009766 & .0009272 & .0003135 & -+- & .0005669 & .0003342 & .0000751 & $\ldots$ & .0003516 & .0001650 & .0000307 \\
\hline $\begin{array}{l}\text { 3bddee -. } \\
\text { bbDdee -- }\end{array}$ & -... & .0004883 & .0006360 & .0003638 & $-\ldots$ & 2834 & 92 & & & 1758 & .0001615 & .0000582 \\
\hline $\begin{array}{l}\text { MabbDdee -- } \\
\text { AnbbddIe -- }\end{array}$ &.- & 004883 & .0006360 & .000 & ----- & 002834 & .000 & & & & & \\
\hline $\begin{array}{l}\text { AnbbddIE -- } \\
\text { AaBBddee -- }\end{array}$ & --- & 0004883 & .000 & .0003638 & $\ldots$ & 1002834 & & & & .000175 & .0001615 & \\
\hline $\begin{array}{l}\text { Baddee -. } \\
\text { bbodee -. }\end{array}$ & 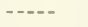 & .0004883 & .0006360 & .0003638 & 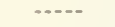 & 002834 & .0002892 & .0001206 & & 758 & & \\
\hline bbDDee -. & $\ldots$ & .0004883 & .0006360 & .0003638 & -.-- & 002834 & .0002892 & & & .000175 & .0001 & \\
\hline bbddez -- & 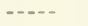 & .0004883 & .0006360 & .0003638 & $-\ldots-$ & 002834 & .0002892 & .0001 & & .0001758 & .00016 & \\
\hline BBDdee -- & -... & .0004883 & .0006360 & .0003638 & 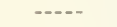 & 1002834 & .0002892 & .0001206 & $\ldots$ & & .0001 & \\
\hline 3addRe - - & $\ldots$ & .0004883 & .0006360 & .00 & $\ldots$ & 0002834 & .0002892 & & -.... & .0001758 & .0001 & \\
\hline $\begin{array}{l}\text { 3b DDee -. } \\
\text { Bbd dEZ -- }\end{array}$ &.- & .0004883 & .0006360 & .000 & $-\cdots$ & 002834 & .0002892 & .0001206 & & .0001758 & & \\
\hline BbddEZ -- & -- & .0004883 & .0006360 & .0003638 & $-\ldots$ & 002834 & .0002892 & & --- & & .0001 & \\
\hline $\begin{array}{l}\text { AabbDDEe -- } \\
\text { AabbDdEE -- }\end{array}$ & $\cdots$ & .0004883 & .0006360 & .0003638 & $\ldots$ & 0002834 & .000 & .0001206 & & .0001758 & .0001 & \\
\hline$\triangle a D b D d E Z=-$ & $\cdots$ & .0006883 & .0006360 & .0003638 & $+\ldots$ & .0002834 & .0002892 & .0001206 & & .0001758 & .0001615 & \\
\hline bDdZe - -. & 5000000 & .183 & & & 4444443 & 1043 & & & .3750000 & & & 495 \\
\hline bddee -- & $\ldots$ & 441 & & & $\ldots$ & 1417 & & & - - & & & \\
\hline Bddee -- & $\cdots$ & 0002441 & .0005608 & .0005358 & $\ldots$ & & & & & & & \\
\hline aabbDDee -- & -.... & .0002441 & .0005608 & .0005358 & $-\cdots$ & 1417 & .0003083 & .0002361 & & & & \\
\hline aabbddzz -- & $-\ldots$ & .0002441 & .0005608 & .0005358 & $\ldots$ & .0001417 & .0003083 & .0002361 & & .0000879 & .0001881 & .0001311 \\
\hline Bobdee =- & $\ldots$ & & & & $--\cdot$ & & & &.-- & & & \\
\hline BbddZe-- & $\ldots$ & 214844 & & & -.... & & & & & & & \\
\hline AabbDdZe -- & -.... & .0214844 & .0198931 & .00799 & $\ldots$ & 92744 & & & & & & \\
\hline aaBoDdZe -. & $\ldots$ & .0214844 & .0198931 & .0079903 & $\ldots$ & .0192744 & .0104840 & .0029845 & & .0161719 & .0067105 & .001626 \\
\hline Bbddee -- & $-\cdots$ & .02 & & & & .01 & & & & & & .0050155 \\
\hline AabbDdee -- & $\cdots$ & 205078 & & & $\ldots .$. & & & & & & & \\
\hline Aabbddze -- & -...- & .0205078 & .0213091 & .0142795 & $-\ldots$ & & .0 & .0 & & .0158 & & \\
\hline aa BbDdee -- & -..- & .0205078 & .0213091 & .0142795 & $\ldots$ & 87075 & .0146196 & .00760 & & & & \\
\hline aa3oddze -- & $\cdots$ & .0205078 & .0213091 & .0142795 & -.... & .0187075 & .0146196 & & & .0 & & \\
\hline aabbDdze -- & $\ldots$ & .0205078 & .0213091 & .0142795 & $\cdots$ & .0187075 & .0146196 & .0076000 & -...- & .0168203 & .0108799 & \\
\hline lee -- & $-\ldots$ & 200195 & .035 & & $\ldots+$ & & & & & & .02 & .021 \\
\hline aabbddee-- & 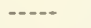 & .0200195 & .0356034 & & & & 298616 & .02 & & & & \\
\hline aabbDdee -- & ....- & .0200195 & .0356034 & .0389054 & ----- & .0184240 & .0298616 & .027915 & & & 244767 & .0214194 \\
\hline aabbddRe -- & 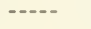 & .0200195 & .0356034 & .0389054 & $\ldots$ & .0184240 & .0298616 & .0279153 & $\ldots$ & .0156445 & .0244767 & .0214194 \\
\hline & 2500000 & .4104004 & .5650664 & .7062349 & .4444443 & .5987812 & .7230061 & .8252550 & .5625000 & .6961816 & .7961094 & .8751972 \\
\hline
\end{tabular}


Table 38,--Relative frequenctes of genotypes when native population (AABBOOEE) is overflooded in parental, $F_{1}, F_{2}$, and $F_{3}$ generations by $\underline{x}$ release insects (aabbddee) for every native insect--Continued

\begin{tabular}{|c|c|c|c|c|c|c|c|c|c|c|c|c|c|}
\hline \multirow[b]{2}{*}{ Genotype } & & & $\underline{x}$ & 4 & & & $\underline{x}$ & 5 & & & $\underline{x}$ & 10 & \\
\hline & & $\mathrm{F}_{1}$ & $\mathrm{~F}_{2}$ & $\mathrm{~F}_{3}$ & $\mathrm{~F}_{4}$ & $F_{1}$ & $\mathrm{~F}_{2}$ & $\bar{F}_{3}$ & $F_{4}$ & $\mathrm{~F}_{1}$ & $F_{2}$ & $\mathrm{~F}_{3}$ & $\mathrm{~F}_{4}$ \\
\hline AABBOOEE & & & .00053250 & 0.00000480 & 0.0000000 & .0277778 & 0.0002865 & 0.0000022 & 0.0000000 & 0.0082645 & 0.00004350 & .0000002 & 00000000 \\
\hline & & & .0003550 & .0000107 & .0000002 & & .0002204 & .0000056 & .0000001 & & .0000484 & .0000008 & .0000000 \\
\hline & -- & $\cdots$ & .0003550 & .0000107 & .0000002 & & .0002204 & .0000056 & .0000001 & & .0000484 & .0000008 & .0000000 \\
\hline AABB $00 E E$ & $\cdots$ & & .0003550 & .0000107 & .0000002 & & .0002204 & .0000056 & .0000001 & $-\cdots$ & .0000484 & .0000008 & .0000000 \\
\hline Aa BBDOEE & -- & & .0003550 & .0000107 & .0000002 & & .0002204 & .0000056 & .00000 & --- & .0000484 & .0000008 & .0000000 \\
\hline AABBDDee & $=$ & & .0000592 & .0000061 & .0000003 & & .0000424 & .0000035 & .0000001 & & .0000134 & .0000007 & .0000000 \\
\hline$A A B B d d E E$ & $=$ & $\cdots$ & .0000592 & .0000061 & .0000003 & & .0000424 & .0000035 & & & .0000134 & .0000007 & .0000000 \\
\hline AAbb DDEE & $=$ & $\cdots$ & .0000592 & .0000061 & .0000003 & $\cdots$ & .0000424 & .0000035 & .00000 & $\cdots$ & .0000134 & .0000007 & .0000000 \\
\hline aABBOOEE & & & .0000592 & .0000061 & .0000003 & & .0000424 & .0000 & .0000 & & .0000134 & & .0000000 \\
\hline AABBOdEe & -- & $\ldots$ & .0004734 & .0000320 & .0000012 & & 0003052 & .0000181 & .0000006 & & .0000752 & .0000032 & .0000001 \\
\hline$\triangle A B b D 0 E e$ & -- & $\cdots$ & .0004734 & .0000320 & .0000 & $\cdots$ & 003052 & .0000181 & .0000 & & .0000752 & .000 & .000 \\
\hline AABb OdEE & -- & -... & .0004734 & .0000320 & .0000 & $\ldots$ & 003052 & .0000181 & .0000 & ---- & .0000752 & .0000 & .000 \\
\hline $\mathrm{AaBB} 00 \mathrm{Ee}$ & -- & ---- & .0004734 & .0000 & & & 03052 & .0000 & .0000 & & .0000752 & .000 & 01 \\
\hline AABBODEE & -- & $\cdots$ & .0004734 & .0000320 & & & 03052 & .0000 & .0000 & $\cdots$ & .0000752 & .0000 & 001 \\
\hline AaBbODEE & -- & ...... & & .0000 & & 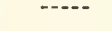 & .0003052 & .000 & & $-\cdots$ & .0000752 & .000 & \\
\hline AAB BOdee & -- & -... & .0001183 & .0000225 & .0000019 & - & .0000848 & .0000140 & .0000011 & & .0000269 & .0000032 & .0000002 \\
\hline AABBddEe & -- &.-- & .0001183 & .00002 & & & 000848 & .0000 & .0000 & & .0000269 & .0000 & .00 \\
\hline ABbDOee & -- & $\cdots$ & .0001183 & .0000225 & & $\ldots$ & .0000848 & .00001 & .0000 &.--- & .0000269 & .0000 & .00 \\
\hline BbddEE & - & $\cdots$ & & .0000 & & $\cdots$ & 00848 & & &..- & .0000269 & .0000 & \\
\hline AbbOOEe & -- & $\cdots$ & 1183 & .0000 & & $\ldots$ & 0848 & & & & .0000269 & .000 & \\
\hline Abb DdEE & -- & --- & & .00002 & & $\cdots$ & 00848 & & & & .0000269 & .000 & \\
\hline BBDOee & -- & $\ldots$ & 01183 & .0000 & & & 000848 & .0 & & --- & .0000269 & .000 & 02 \\
\hline BBddEE & -- & $\cdots$ & & .0000225 & .00000 & & .0000848 & .0000 & .000 & -... & .0000269 & .0000 & 02 \\
\hline BbOOEE & $\cdots$ & $-\cdots$ & 01183 & .0000 & & -- & 00848 & .000 & & -..... & .0000269 & .000 & \\
\hline BBOOEe & -- & $\ldots$ & 01183 & .0000 & & & 0848 & .00 & & & .0000 & .000 & \\
\hline BBOdEE & -- & - & 183 & .0000 & .00 & 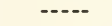 & 000848 & .000 & & ---- & .0000 & .000 & 02 \\
\hline aaBb00EE & -- & -- & .0001183 & .0000225 & .0000 & $\cdots+$ & 00848 & .0000 & .0000 & $-\ldots-$ & .0000 & .000 & \\
\hline & -- & - & & 0001164 & & - & 04747 & 02 & & & 0001290 & 147 & \\
\hline BBDdEe & -- & & & .000 & & & 4747 & 2 & & & & & \\
\hline BbDOEe & -- & - & 101 & .000 & .000 & $\cdots$ & 004747 & .0000 & .000 & -.... & .0001290 & .000 & 09 \\
\hline AaBbOdEE & -- & $\cdots$ & .0007101 & .0001164 & .0000091 & --..- & .0004747 & .0000702 & .0000051 & -... & .0001290 & .0000147 & .000 \\
\hline & -- & - & .0000592 & .0000209 & 0000035 & - & .0000424 & .0000139 & .0000 & & .0000134 & .000 & 2005 \\
\hline & -- & & 592 & .000 & & & 9424 & .000 & & & 34 & & \\
\hline bbddEE & -- & - & 1592 & .0000 & .000 & $\cdots$ & 00424 & .000 & .000 & 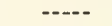 & .0000 & .000 & \\
\hline & -- & & .0000592 & .000 & & & 0424 & .000 & & & & & \\
\hline aABBddEE & - & & .0000592 & .0000 & .00 & & 00424 & .000 & .00 & $--\cdot-$ & .0000134 & .000 & .00 \\
\hline aabb0DEE & -- & & .0000592 & .0000209 & .0000035 & & .0000424 & .0000139 & .0000021 & $-\cdots$ & 134 & .00 & .00 \\
\hline & - & - & .0002367 & .0000969 & .0000 & $\ldots$ & .0001695 & .0000 & .0000 & & .0000537 & .0000 & .00 \\
\hline & -- & & & & & & 1695 & .000 & & & .000 & .00 & \\
\hline dEe & -- & - & & & & & 1695 & .00 & & ---- & .000 & & \\
\hline dee & -- & & & & & & 1695 & .000 & & & & & \\
\hline $\mathrm{dEe}$ & $\ldots$ & $\cdots$ & .0002367 & .00009 & .000 & -- & 1695 & .0000 & & & .0000 & & \\
\hline Oee & -- & & & .000 & & -.... & 1695 & & & & .0000637 & & \\
\hline & -- & & 367 & .000 & & & 1695 & & & & & & \\
\hline & -- & $\cdots$ & & .000 & & - & 1695 & .00 & &.-- & 000637 & .0 & .00 \\
\hline AEE & -- & & & .000 & & & 1695 & .000 & & & .000 & & \\
\hline & - & $\cdots$ & 0002367 & .00009 & .000 & $\ldots$ & .0001695 & .0000634 & .0000 & $-\ldots$ & .0000637 & .000 & .00 \\
\hline $\mathrm{a} a \mathrm{BbI}$ & -- & $-\ldots$ & .0002367 & .000 & .000 & -..- & 1695 & .0000634 & & & .0000637 & .000 & 021 \\
\hline$a a B b O d E E$ & -- & & .0002367 & .0000969 & .0000161 & & .0001695 & .0000634 & .0000097 & & .0000637 & .0000163 & .0000021 \\
\hline & -- & - & & & & & & & & & & & \\
\hline & -- & -... & & .000 & & --- & 0848 & & & & & & \\
\hline AAbbd & -- & & & .000 & & --- & 0848 & .000 & &...-- & & & \\
\hline & -- & & & .000 & & & 848 & .000 & & & & & \\
\hline & -- & - & & & & --- & 0848 & .000 & & --..- & 00269 & .000 & 467 \\
\hline $\mathrm{ddEE}$ & -- & & & & & & & & & & & & \\
\hline & -- & - & 1183 & .000 & & $-\ldots$ & 0848 & .000 & & & 269 & & \\
\hline & -- & & & & & & 0848 & & & -..-- & & & \\
\hline & -- & $-\cdots$ & & & & & & & & & & & \\
\hline & -. & & & .000 & & - & 848 & .00 & &..-- & 269 & & \\
\hline & -- & & & & & & & .0000 & & & & .000 & 467 \\
\hline a abbOdEE & -- & - & .0001183 & .0001025 & .0000339 & & .0000848 & .0000707 & .0000221 & & .0000269 & .0000206 & .0000467 \\
\hline & & 00000 & & & & 2777777 & & & & 1652892 & & & \\
\hline & -- & & & & & -- & & & & $\ldots$ & & & \\
\hline & -- & & & & & & & & & & & & \\
\hline & -- & - & & & & $\ldots$ & & & & -... & & & \\
\hline aabbddEE & -- & & .0000592 & .0001257 & .0000831 & $\ldots$ & .0000424 & .0000897 & .0000573 & $\ldots$ & .0000134 & 282 & .0000168 \\
\hline & $=$ & & & & & $\cdots$ & & & & & & & \\
\hline & -- & & & & & & & & & & & & \\
\hline & -- & & & & & 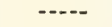 & & & & - - - & & & \\
\hline & -- & -... & .0137278 & .0048131 & .0010579 & $-\ldots$ & .0118679 & .0037046 & .0007615 & -... & .0069874 & .0016477 & .0002874 \\
\hline & -- & & & & & - & & & & & & & \\
\hline & & & & & & & & & & & & & \\
\hline & -- & & & & & $-\cdots$ & & & & $\ldots$ & & & \\
\hline & -- & -.... & & & & ---- & & .007 & & $-\ldots$ & & & \\
\hline & -- & & & & & & & & & $-\infty$ & & & \\
\hline aabbDdEe & -- & $\cdots$ & .0134911 & .0086192 & .0036980 & & .0116984 & .0071226 & .0029122 & $\cdots$ & .0069336 & .0037924 & .0013869 \\
\hline & -- & & & & & & & & & & & & \\
\hline & -- & & .33728 & & & & & & & & & & \\
\hline & -- & & 37278 & & & - . - & & & & 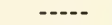 & & & \\
\hline a abbddEe & -- & & .0133728 & .0205367 & & --D- & .0116136 & & & --- & & & .0080244 \\
\hline tovdare & & & & & & 4444 & & & & 6446 & & 058 & \\
\hline
\end{tabular}




\begin{tabular}{|c|c|c|c|c|c|c|c|c|c|c|c|c|c|}
\hline \multirow{2}{*}{ ienotype } & & & $\underline{x}$ & 20 & & & $\underline{x}$ & 50 & & & $\underline{x}$ & 100 & \\
\hline & & $E_{1}$ & $F_{2}$ & $\mathrm{~F}_{3}$ & $\mathrm{~F}_{4}$ & $F_{1}$ & $F_{2}$ & $F_{3}$ & $F_{4}$ & $F_{1}$ & $F_{2}$ & $F_{3}$ & $F_{4}$ \\
\hline AABBDDEE & $=0.0$ & 0222676 & 0.0000075 & 0.0000000 & 0.0000000 & $0.0003=45$ & 0.0000009 & 0.0000000 & 0.0000000 & 0.0000980 & 0.0000002 & 0.0000000 & 0.0000000 \\
\hline AABBDDEe - & & & .0000107 & .0000001 & .0000000 & -...- & .0000015 & .0000000 & .0000000 & $\cdots$ & .0000004 & .0000000 & .0000000 \\
\hline MBBDAEE - & -- & & .0000107 & .0000001 & .0000000 & & .0000015 & .0000000 & .0000000 & $-\infty$. & .0000004 & .0000000 & .0000000 \\
\hline MBSBDEE - & & $\ldots$ & .0000107 & .0000001 & .0000000 & - & .0000015 & .0000000 & .0000000 & -...- & .0000004 & .0000000 & .0000000 \\
\hline AS 3BODEE - & & $\cdots$ & .0000107 & .0000001 & .0000000 & $-\infty$ & .0000015 & .0000000 & .0000000 & - n- & .0000004 & .0000000 & .0000000 \\
\hline MBs Dilee - & -- & -.... & .0000036 & .0000001 & .0000000 & ---- & .0000007 & .0000000 & .0000000 & $-\infty-\infty$ & .0000002 & .0000000 & .0000000 \\
\hline & -- &.-- & .0000035 & .0000001 & .0000000 & & .0000007 & .0000000 & .0000000 & $-\ldots$ & .0000002 & .0000000 & .0000000 \\
\hline AAB BDEEE - & -- & & .0000038 & .0000001 & .0000000 & -n. & .0000007 & .0000000 & .0000000 & $-\infty-\infty$ & .0000002 & .0000000 & .0000000 \\
\hline & $-\infty$ & $\ldots$ & .0000038 & .0000001 & .0000000 & ---- & .0000007 & .0000000 & .0000000 & $-\infty-\infty$ & .0000002 & .0000000 & .0000000 \\
\hline MBBAdEe - & -- & & .0000153 & .0000006 & .0000000 & - & .0000028 & .0000001 & .0000000 & -... & .0000007 & .0000000 & .0000000 \\
\hline DEe - & -- & $\ldots$ & .0000183 & .0000006 & .0000000 & $-\cdots$ & .0000028 & .0000001 & .0000000 & & .0000007 & .0000000 & .0000000 \\
\hline dEE . & -- & $-\infty-$ & .0000183 & .0000006 & .0000000 & 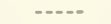 & .0000028 & .0000001 & .0000000 & $-\cdots$ & .0000007 & .0000000 & .0000000 \\
\hline DEe - & -- & $-\ldots$ & .0000183 & .0000006 & .0000000 & -.... & .0000028 & .0000001 & .0000000 & $\ldots$. & .0000007 & .0000000 & .0000000 \\
\hline A3BDdEE - & -- & $---\infty$ & .0000183 & .0000006 & .0000000 & $-\cdots$ & .0000028 & .0000001 & .0000000 & & .0000007 & .0000000 & .0000000 \\
\hline A $360 \mathrm{DEE}$ & - & $\ldots+$ & .0000183 & .0000006 & .0000000 & $-\infty$ & .0000028 & .0000001 & .0000000 & --- & .0000007 & .0000000 & .0000000 \\
\hline AMBBDdee - & - & $-\infty-$. & .0000076 & .0000007 & .0000000 & $=-\infty$ & .0000013 & .0000001 & .0000000 & & .0000003 & .0000000 & .0000000 \\
\hline MABBdAEE - & -- & -.... & .0000076 & .0000007 & .0000000 & ---- & .0000013 & .0000001 & .0000000 & $-\cdots$ & .0000003 & .000 & .0000000 \\
\hline MabODee - & $-\infty$ & -.... & .0000076 & .0000007 & .0000000 & ---- & .0000013 & .0000001 & .0000000 & $-\ldots$ & .0000003 & .0000000 & .0000000 \\
\hline MABbddEE . & $\ldots$ & -... & .0000076 & .0000007 & .0000000 & & .0000013 & .0000001 & .0000000 & $\cdots$ & .0000003 & .0000000 & .0000000 \\
\hline MBbDDEe - & -- & ..... & .0000076 & .0000007 & .0000000 & $-\ldots-$ & .0000013 & .0000001 & .0000000 & & .0000003 & .0000000 & .0000000 \\
\hline ANbb AdEE - & -- & $\ldots$ & .0000076 & .0000007 & .0000000 & $=-\ldots$ & .0000013 & .0000001 & .0000000 & & .0000003 & .000 & 0000 \\
\hline AABBDDee - & -- & $\cdots$ & .0000076 & .0000007 & .0000000 & -...- & .0000013 & .0000001 & .0000000 & ....... & .0000003 & .000 & .0000000 \\
\hline AABBdAEE : & $\therefore-$ & $\ldots$ & .0000076 & .0000007 & .0000000 & -.... & .0000013 & .0000001 & .0000000 & & .0000003 & .0000000 & .000 \\
\hline Aabb DoEE - & - & - & .0000076 & .0000007 & .0000000 & $\ldots$ & .0000013 & .0000001 & .0000000 & & .0000003 & .000 & .000 \\
\hline A33DOEe - & -- & -.... & .0000076 & .0000007 & .0000000 & -.... & .0000013 & .0000001 & .0000000 & & .0000003 & .0000000 & .0000000 \\
\hline ABBDders - & -- & $-\ldots$ & .0000076 & .0000007 & .0000000 & $\ldots$ & .0000013 & .0000001 & .0000 & & .0000003 & .0000000 & .0000000 \\
\hline a 3 bODEE - & - & $\cdots$ & .0000076 & .0000007 & .0000000 & $\cdots$ & .0000013 & .0000001 & .0000000 & & .0000003 & .0000000 & .0000 \\
\hline dEe . & -- & -+-- & .0000335 & .0000032 & .0000002 & & .0000055 & .0000005 & .0000000 & & .0000014 & .0000001 & .0000000 \\
\hline 3BDdEe - & -- & -...- & .0000335 & .0000032 & .0000002 & $--\cdot$ & .0000055 & .0000005 & .0000000 & & .0000014 & .0000001 & .0000000 \\
\hline Al3bDoce - & -- & 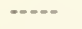 & .0000335 & .0000032 & .0000002 & $+\infty$ & .0000055 & .0000005 & .0000000 & 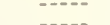 & .0000014 & .0000001 & .0000000 \\
\hline MESODCEE . & - & -...- & .0000335 & .0000032 & .0000002 & - - - & .0000055 & .0000005 & .0000000 & & .0000014 & .0000001 & .000 \\
\hline dee. & - & - & .0000038 & .0000010 & .0000001 & ---- & .0000007 & .0000002 & .0000000 & & .0000002 & .0000000 & .0000000 \\
\hline & -- & $=\ldots$ & .0000038 & .0000010 & .0000001 & & .0000007 & .0000002 & .0000000 & & .0000002 & .0000 & .0000000 \\
\hline AAbbddEE - & -- & ---- & .0000038 & .0000010 & .0000001 & $-\cdots$ & .0000007 & .0000002 & .0000000 & & .0000002 & .0000000 & .0000000 \\
\hline 3BODee - & - & ---- & .0000038 & .0000010 & .0000001 & & .0000007 & .0000002 & .0000000 & & .0000002 & .0000000 & .0000000 \\
\hline AB Bd dEE & - & $+\infty$ & .0000038 & .0000010 & .0000001 & $-\ldots$ & .0000007 & .0000002 & .0000000 & & .0000002 & .0000000 & .0000000 \\
\hline AabbDOEE - & -- & $\ldots$ & .0000038 & .0000010 & .0000001 & - & .0000007 & .0000002 & .0000000 & & .0000002 & .0000000 & .0000000 \\
\hline ABbDdee - & -- & $-\ldots$ & .0000152 & .000041 & .0000005 & ---- & .0000026 & 0006 & .0000001 & & .0000007 & .000 & .0000000 \\
\hline MBbddEe - & -- & $+\ldots$ & .0000152 & .0000061 & .0000005 & $-\infty$ & .0000026 & .0000006 & .00000 & & .0000007 & .000 & 0000 \\
\hline obDdEe - & -- & --- & .0000152 & .0000041 & .0000005 & $\ldots$ & .0000026 & .0000006 & .0000001 & & .0000007 & .0000002 & .0000000 \\
\hline BBDdee - & -- & $\ldots$ & .0000152 & .0000041 & .0000005 & --- & .0000026 & .0000006 & .0000001 & & .0000007 & .0000002 & .0000000 \\
\hline diEe - & -- & $\ldots$ & .0000152 & .0000042 & .0000005 & $\ldots$. & .0000026 & .0000006 & .0000001 & on- & .0000007 & .0000002 & .0000000 \\
\hline SbODee - & - & c.... & .0000152 & .0000041 & .0000005 & $\ldots$ & .0000026 & .0000006 & .0000001 & & .0000007 & .0000002 & .0000000 \\
\hline ALBbddES - & -- & $\ldots$ & .0000152 & .0000041 & .0000005 & & .0000026 & .0000006 & .000000 & & .0000007 & .0000002 & .0000000 \\
\hline bODEe - & -- & ..... & .0000152 & .0000041 & .0000005 & $-\cdots$ & .0000026 & .0000006 & .0000001 & $-\infty$ & .0000007 & .0000002 & .0000000 \\
\hline dEE = & -- & $\ldots$ & .0000152 & .0000041 & .0000005 & & .0000026 & .0000006 & .0000001 & & .0000007 & .0000002 & .0000000 \\
\hline Aze - & -- & 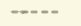 & .0000152 & .0000041 & .0000005 & $+\cdots$ & .0000026 & .0000006 & .0000001 & & .0000007 & .00 & .0000000 \\
\hline$\triangle A B b O D E e$ & -- & $\ldots$ & .0000152 & $.00000<1$ & .0000005 & ---- & .0000026 & .0000006 & .0000001 & - & .0000007 & .0000002 & .0000000 \\
\hline A ABDDdEE & -- & $\ldots$ & .0000152 & .0000041 & .0000005 & $\ldots$ & .0000026 & .0000006 & .0000001 & & .0000007 & .0000002 & .0000000 \\
\hline & -- & ----- & & .0000 & 0014 & & 0013 & 0009 & .000 & & .0000003 & .000 & .0000000 \\
\hline & -- & $-\cdots$ & .0000076 & .0000056 & .0000014 & & .0000013 & .0000009 & .0000 & & .0000003 & .000 & .0000000 \\
\hline MbbddEe - & -- & $\ldots$ & .0000076 & .0000056 & .0000014 & $=-$. & .0000013 & .0000009 & .0000002 & & .0000003 & .0000002 & .0000000 \\
\hline dee - & -- & $\ldots+\infty$ & .0000076 & .0000056 & .0000014 & $\cdots$ & .0000013 & .0000009 & .0000002 & -1 & .0000003 & .000 & .0000000 \\
\hline & -- & $-\cdots$ & .0000076 & .0000056 & .0000014 & & .0000013 & .0000009 & .0000002 & & .0000003 & .0000002 & .0000000 \\
\hline AabbddeE - & - & -....- & .0000076 & .0000056 & .0000014 & 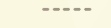 & .0000013 & .000000 & .0000002 & -... & .0000003 & .0000002 & .0000000 \\
\hline dee. & $-\cdot$ & $-\infty+$ & .0000076 & .0000056 & .0000014 & & .0000013 & .0000009 & .0000002 & --.-- & .0000003 & .0000002 & .0000000 \\
\hline dEe - & -- &.-- & .0000076 & .0000056 & .0000014 & -1. & .0000013 & .0000009 & .000000 & & .0000003 & .0000002 & .0000000 \\
\hline Dee - & -- & $-\infty-\infty$ & .0000076 & .0000056 & .0000014 & & .0000013 & .0000009 & .0000002 & $\cdots$ & .0000003 & & 2000 \\
\hline IdEE - & -- & $\ldots--$ & .0000076 & .0000056 & .0000014 & $\cdots$ & .0000013 & .0000009 & .0000002 & 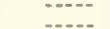 & .0000003 & .0000002 & .0000000 \\
\hline asbbDEe & -. & $\cdots$ & .0000076 & .0000056 & .0000014 & & .0000013 & .0000009 & .0000002 & & .0000003 & .0000 & .0000000 \\
\hline $2 a b b D d \bar{C}$ & $-\cdot$ & $-\ldots$ & .0000076 & .0000056 & $.000001<$ & -- & .0000013 & .0000009 & .0000002 & & .0000003 & .0000002 & .0000000 \\
\hline $18 e$. & -.0 & 907029 & 053536 . & .0003603 & & 84667 & .0018689 & .000 & & 1296059 & & & \\
\hline & -- & -.... & 0000038 & .0000080 & .0000046 & & .0000007 & .000 & & & & & \\
\hline 3Bddee & -- & $-\ldots$ & .0000038 & .0000080 & .0000046 & -- & .0000007 & 0014 & .000 & & .0000002 & .0000004 & 0002 \\
\hline aabbDDee & -- & $-\ldots$ & .0000038 & .0000080 & .0000046 & 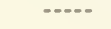 & .0000007 & .000 & .000 & - & .0000002 & .0000004 & 0002 \\
\hline aabbddEC & -- & - . & .0000038 & .0000080 & .0000046 & $--\cdot-$ & .0000007 & .0000014 & .0000008 & & .0000002 & .0000004 & .0000002 \\
\hline lee & - & $-\ldots$ & 0038087. & & &...- & & & .0000 & & & & \\
\hline & -- & $-\ldots$ & .0038087 & .0007544 & .0001182 & & .0016071 & .0002822 & .0000408 & -.... & & & \\
\hline Mabbidz̃e & -. & ..... & .0038087 & .0007544 & .0001182 & -.... & 16071 & .0002822 & .0001 & $-\ldots$ & .0008183 & .0001376 & .0000193 \\
\hline aaBbidEe & $-\infty$ & - - & .0038087 & .0007546 & .0001182 & & .0016071 & .0002822 & .0000408 &.--- & .0008183 & .0001376 & .0000193 \\
\hline lee & & & & .00195 & 6688 & $\ldots$ & .0016045 & & & - - & & & \\
\hline & -- & & .0037935 & .0019535 & 006688 & -... - & .0016045 & .0007948 & .0002 & $\ldots$ & .00081 & & \\
\hline AabbddEe & -. & $=-$. & .0037935 . & .0019535 & .0006688 & -...- & .0016045 & .0007948 & .00026 & -...- & .0008176 & 996 & .000 \\
\hline & -- & ..... & .0037935 . & .0019535 & .0006688 & ..... & .0016045 & .0007948 & .000 & $\ldots$ & .0008176 & .0003996 & .0001289 \\
\hline & -. & $\ldots .$. & .0037935 . & .0019535 & .0006688 & -... & .0016045 & .0007948 & .0002605 & --- & .0008176 & 96 & .0001289 \\
\hline aabbDdEe & -- & .... & .0037935. & .0019535 & .0006688 & - - - & .0016045 & .0007948 & .0002605 & - - - & .0008176 & .0003996 & .0001289 \\
\hline bbddee. & $\ldots$ & -... & .0037859 . & & & -...- & & & & $\ldots$ & & & \\
\hline & -- & & .0037855 . & .0055448 & .0042286 & & .0016032 & 3306 & & -.... & .0008172 & .0011 & .0008834 \\
\hline bbodee & -- & .... & .0037859 & .0055448 & .0042286 & $\ldots$ & .0016032 & .0023306 & .0017474 & $\ldots$ & .0008172 & .0011851 & .0008834 \\
\hline aabbidze & - & $-\cdots$ & .0037855 & .0055448 & .0042286 & -1 & .0016032 & .0023306 & .0017474 & $\ldots$ & .0008172 & .0011851 & .0008834 \\
\hline abbiddee- & $=.90$ & 070289 & $.940794 \mathrm{C}$. & .9625418 & .9785320 & 511688 & .9755442 & 9846391 & .9912704 & 2959 & & & \\
\hline
\end{tabular}


Table 3B.--Relative frequencies of genotypes when native population (AABBDDEE) is overflooded in parental, $F_{1}$, $F_{2}$, and $F_{3}$ generations by $\underline{X}$ release insects (aabbddee) for every native insect--Continued

\begin{tabular}{|c|c|c|c|c|c|c|c|c|c|c|c|c|}
\hline \multirow{2}{*}{ Genotype } & & $\underline{x}=$ & $=200$ & & & $\underline{X}$ & 500 & & & $\underline{X}$ & 1,000 & \\
\hline & $\mathrm{F}_{1}$ & $\mathrm{~F}_{2}$ & $\mathrm{~F}_{3}$ & $F_{4}$ & $F_{1}$ & $F_{2}$ & $F_{3}$ & $F_{4}$ & $F_{1}$ & $\mathrm{~F}_{2}$ & $F_{3}$ & $\mathrm{~F}_{4}$ \\
\hline $\mathrm{AABBDDEE}_{-0.0}$ & $00024 B$ & 0.0000000 & 0.0000000 & 0.0000000 & 0.0000040 & 0.0000000 & 0.0000000 & 0.0000000 & 0.0000010 & 0.00000000 & 0.0000000 & 0.0000000 \\
\hline AABBDDEe - & $=-\infty$ & .0000001 & .0000000 & .0000000 & -..... & .0000000 & .0000000 & .0000000 & & .0000000 & .0000000 & .0000000 \\
\hline AABBDdEE -- & $-\cdots$ & .0000001 & .0000000 & .0000000 & --- & .0000000 & .0000000 & .0000000 & -..-- & .0000000 & .0000000 & .0000000 \\
\hline AAB BDDEE -- & $m-n$ & .0000001 & .0000000 & .0000000 & $-\infty$ & .0000000 & .0000000 & .0000000 & & .0000000 & .0000000 & .0000000 \\
\hline AaBBDDEE -- & & .0000001 & .0000000 & .0000000 & $\ldots$ & .0000000 & .0000000 & .0000000 & ---- & .0000000 & .0000000 & .0000000 \\
\hline AABBDDee -- & - - & .0000000 & .0000000 & .0000000 & $-\ldots$ & .0000000 & .0000000 & .0000000 & & .0000000 & .0000000 & .0000000 \\
\hline AABBddEE -- & $\cdots$ & .0000000 & .0000000 & .0000000 & $-\ldots$ & .0000000 & .0000000 & .0000000 & & .0000000 & .0000000 & .0000000 \\
\hline AAbbDDEE -- & -...- & .0000000 & .0000000 & .0000000 & & .0000000 & .0000000 & .0000000 & & .0000000 & .0000000 & .0000000 \\
\hline a aBBDDEE -- & $\ldots$ & .0000000 & .0000000 & .0000000 & -... & .0000000 & .0000000 & .0000000 & & .0000000 & .0000000 & .0000000 \\
\hline AABBDdEe -- & $\cdots-$ & .0000002 & .0000000 & .0000000 & --- & .0000000 & .0000000 & .0000000 & & .0000000 & .0000000 & .0000000 \\
\hline AABbDDEe -- & $\cdots$ & .0000002 & .0000000 & .0000000 & $\cdots$ & .0000000 & .0000000 & .0000000 & $-\ldots$ & .0000000 & .0000000 & .0000000 \\
\hline AABbDdEE -- & $\ldots$ & .0000002 & .0000000 & .0000000 & -.... & .0000000 & .0000000 & .0000000 & ---- & .0000000 & .0000000 & .0000000 \\
\hline AaBBDDEe -- & $\ldots$ & .0000002 & .0000000 & .0000000 & $-\cdots$ & .0000000 & .0000000 & .0000000 & & .0000000 & .0000000 & .0000000 \\
\hline AaBBDdEE -- & --- & .0000002 & .0000000 & .0000000 & 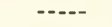 & .0000000 & .0000000 & .0000000 & & .0000000 & .0000000 & .0000000 \\
\hline AaBbDDEE -- & $-\cdots$ & .0000002 & .0000000 & .0000000 & --- & .0000000 & .0000000 & .0000000 & & .0000000 & .0000000 & .0000000 \\
\hline AABBDdee -- & 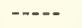 & .0000001 & .0000000 & .0000000 & -... & .0000000 & .0000000 & .0000000 & & .0000000 & .0000000 & .0000000 \\
\hline AABBddEe -- & ---1 & .0000001 & .0000000 & .00000 & $-\ldots$ & .0000000 & .0000000 & .0000000 & & .0000000 & .0000000 & .0000000 \\
\hline AABbDDee -- & ---- & .0000001 & .0000000 & .0000000 & -..-- & .0000000 & .0000000 & .0000000 & -- & .0000000 & .0000000 & 000 \\
\hline AABbddEE -- & -... & .0000001 & .0000000 & .0000000 & $-\cdots$ & .0000000 & .0000000 & .0000000 & & .0000000 & .0000000 & .0000000 \\
\hline AAbbDDEe -- & $\cdots--$ & .0000001 & .0000000 & .000 & $\cdots$ & .0000000 & .000 & .0000 & -.... & .0000000 & 000 & \\
\hline AAbbDdEE -- & $\cdots$ & .0000001 & .0000000 & .0000000 & $\ldots$ & .0000000 & .0000000 & .0000000 & --- & .0000000 & .0000000 & .0000000 \\
\hline AaBBDDee -- & ---- & .0000001 & .0000000 & .0000000 & $\ldots$ & .0000000 & .0000000 & .0000000 & $-\cdots$ & .0000000 & .0000000 & .000 \\
\hline$A a B B d d E E--$ & $n$ & .0000001 & .0000000 & .0000000 & ----- & .0000000 & .0000000 & .0000000 & $\ldots$ & .0000000 & & \\
\hline Aabb DDEE -- & -..... & .0000001 & .0000000 & .0000000 & -...- & .0000000 & .0000000 & .0000000 & --.-- & .0000000 & .0000000 & .0000000 \\
\hline aaBBDDEe -- & $--\infty$ & .0000001 & .0000000 & .0000000 & $-\cdots-$ & .0000000 & .0000000 & .0000000 & --- & .0000000 & & \\
\hline aaBBDdEE -- & $\cdots$ & .0000001 & .0000000 & .0000000 & ----- & .0000000 & .0000000 & .0000000 & ---- & .0000000 & .0000000 & .0000000 \\
\hline aaBbDDEE -- & $\cdots$ & .0000001 & .0000000 & .0000000 & $-\ldots$ & .0000000 & .0000000 & .0000000 & -..- & .0000000 & .0000000 & .0000000 \\
\hline AABbDdEe -- & ---- & .0000003 & .0000000 & .0000000 & $-\ldots$ & .0000001 & .0000000 & .0000000 & -.... & .0000000 & .0000000 & .0000000 \\
\hline AaBBDdEe -- & $-n$ & .0000003 & .0000000 & .000 &.--- & .0000001 & .00 & 00000 & & .0000000 & & 1000 \\
\hline AaBbDDEe -- & $\ldots+$ & .0000003 & .0000000 & .0000000 & --..- & .0000001 & .0000000 & .0000000 & -..-- & .0000000 & .0000000 & .0000000 \\
\hline AaBbDdEE -- & $\cdots$ & .0000003 & .0000000 & .0000000 & ---- & .0000001 & .0000000 & .0000000 & ---- & .0000000 & .0000000 & .0000000 \\
\hline AABBddee -- & --- & .0000000 & .0000000 & .0000000 & -...- & .0000000 & .0000000 & .0000000 & --..- & .0000000 & .0000000 & .0000000 \\
\hline AAbbDDee -- & - & .0000000 & .0000000 & .0000000 & ---- & .0000000 & .0000000 & .0000000 & & .0000000 & & 1000 \\
\hline AAbbddEE -- & $\cdots$ & .0000000 & .0000000 & .0000000 & $\ldots-$ & .0000000 & .0000000 & .0000000 & --.- & .0000000 & .0000000 & .0000000 \\
\hline aa BBDDee -- & $\cdots$ & .0000000 & .0000000 & .0000000 & $-\ldots$ & .0000000 & .0000000 & .0000000 & --- & .0000000 & .0000000 & .0000000 \\
\hline aaBBddEE .. & $\cdots$ & .0000000 & .0000000 & .0000000 & --- & .0000000 & .0000000 & .0000000 & & .0000000 & .0000000 & .0000000 \\
\hline aabbDDEE -- & -...- & .0000000 & .0000000 & .0000000 &.--- & .0000000 & .0000000 & .0000000 & & .0000000 & .0000000 & .0000000 \\
\hline AABbDdee -- & $\cdots$ & .0000002 & .0000000 & & & .0000000 & .00 & 10000 & & .0000000 & .0000000 & .0000000 \\
\hline AABbddEe - - & $\cdots$ & .0000002 & .0000000 & .000 &.- & .0000000 & .0000000 & .0000000 & & .0000000 & .0000000 & .0000000 \\
\hline AAbbDdEe ... & 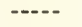 & .0000002 & .0000000 & .000 & --- & .0000000 & .0000000 & .0000000 & & .0000000 & .0000000 & 10000 \\
\hline AaBBDdee -- & $-\ldots$ & .0000002 & .0000000 & .0000000 & & .0000000 & .0000000 & .0000000 & & .0000000 & .0000000 & .0000000 \\
\hline AaBBddEe -- & -- & .0000002 & .0000000 & .0000000 & --- & .0000000 & .0000000 & .0000000 & & .0000000 & 00000 & 0000 \\
\hline AaBbDDee -- & $\cdots$ & .0000002 & .0000000 & .0000000 & $\ldots$ & .0000000 & .0000000 & .0000000 & & .0000000 & .0000000 & .0000000 \\
\hline AaBbddEE -. & $\cdots$ & .0000002 & .0000000 & .0000000 & ---- & .0000000 & .0000000 & .0000000 & & .0000000 & .0000000 & .0000000 \\
\hline AabbDDEe -- & $\cdots$ & .0000002 & .0000000 & .0000000 & --- & .0000000 & .0000000 & .0000000 & & .0000000 & .0000000 & .0000000 \\
\hline AabbDdEE -- & -- & .0000002 & .0000000 & .0000000 & & .0000000 & .0000000 & .0000000 & 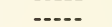 & .0000000 & .0000000 & .0000000 \\
\hline a aBBDdEe -- & --- & .0000002 & .0000000 & .0000000 & ..... & .0000000 & .000 & .0000000 & & .0000000 & 000 & 000 \\
\hline $\mathrm{aaBb}$ DDEe & $-\infty$ & .0000002 & .0000000 & .0000000 & -.... & .0000000 & .0000000 & .0000000 & --.-- & .0000000 & .0000000 & .0000000 \\
\hline aaBbDdEE -- & & .0000002 & .0000000 & .0000000 & $-\ldots$ & .0000000 & .0000000 & .0000000 & & .0000000 & .0000000 & .0000000 \\
\hline AABbddee -- & $\cdots$ & 00001 & .00 & & & .0000000 & .0000000 & .0000000 & & .0000000 & .0000000 & 0000 \\
\hline AAbb Ddee -- & $\cdots$ & .0000001 & .0000001 & & --- & .0000000 & .0000000 & .0000000 & & .0000000 & 000 & 000 \\
\hline AAbbddEe -- & $-\cdots$ & .0000001 & .0000001 & .0000000 & --.-- & .0000000 & .0000000 & .0000000 & & .0000000 & .0000000 & 0000 \\
\hline AaBBddee -- & & .0000001 & .0000001 & .000 & $\ldots$ & .0000000 & .0000000 & .0000000 & & .0000000 & 000 & 10000 \\
\hline AabbDDee -- & $=-\infty$ & .0000001 & .0000001 & .000 & -...- & .0000000 & .0000000 & .0000000 & & .0000000 & .0000000 & .0000000 \\
\hline AabbddEE -- & $\cdots$ & .0000001 & .0000001 & .0000000 & $\ldots$ & .0000000 & .0000000 & .0000000 & $\ldots$ & .0000000 & 000 & .0000000 \\
\hline aаBBDdee -- & -..- & .0000001 & .0000001 & .00000 & --- & .0000000 & .0000000 & .0000000 & & .0000000 & 000 & 0000 \\
\hline aaBBddEe -- & -... & .0000001 & .0000001 & .0000000 & -.... & .0000000 & .0000000 & .0000000 & & .0000000 & .0000000 & .0000000 \\
\hline aaBbDDee -- & & .0000001 & .0000001 & .0000000 & $\ldots$ & .0000000 & .00 & .00 & & 0000 & Doo & 0000 \\
\hline aaBbddEE -- & $\cdots$ & .0000001 & .0000001 & .0000000 & --- & .0000000 & .0000000 & 0000 & & .0000000 & .0000000 & .0000000 \\
\hline aabbDDEe -- & $\cdots$ & .0000001 & .0000001 & & ---- & .0000000 & .0000000 & & - & .0000000 & .0000000 & \\
\hline aabbDdEE -- & & .0000001 & .0000001 & .0000000 & ---- & .0000000 & .0000000 & .0000000 & & .0000000 & .0000000 & .0000000 \\
\hline$e--.0$ & 0099007 & & & & .0039840 & $.000168 \mathrm{~B}$ & & & .0019960 & & & \\
\hline & -- & .0000000 & & & - . & .0000000 & .00 & 0000 & & .0000000 & 000 & 0000 \\
\hline aaBBddee.-. & & .0000000 & .0000001 & & $-\ldots$ & .0000000 & & & & & & \\
\hline aabbDDee -- & --- & .0000000 & .0000001 & .0000000 & ..... & .0000000 & & & & .0000000 & .0000000 & 0000 \\
\hline aabbddEE -- & ---- & .0000000 & .0000001 & .0000000 &.-- & .0000000 & .0000000 & .0000000 & --- & .0000000 & .0000000 & .0000000 \\
\hline $\mathrm{AaBb}$ & -- & 0004129 & & & & .0001661 & & & & & & \\
\hline AaBbddEe -- & --- & .0004129 & .000 & & $-\cdots$ & .0001661 & & & --- & & 134 & \\
\hline AabbDdEe-- & $-\cdots$ & .0004129 & .0000679 & & -.... & .0001661 & .0000269 & .0000037 & & .0000832 & .0000134 & 00018 \\
\hline aaBbDdEe-- & -..- & .0004129 & .0000679 & .0000094 & -- & .0001661 & .0000269 & .0000037 & --- & .0000832 & .0000134 & .0000018 \\
\hline & $\cdots$ & 004127 & & & & 1660 & & & & .000 & 402 & 128 \\
\hline & & .0004127 & .0002004 & & & .0001660 & & & - & & & \\
\hline Aabbdd & - & .0004127 & .0002004 & & $-\ldots$ & .0001660 & $.0000 \mathrm{BO} 3$ & .0000256 & & .0000832 & 00402 & 0128 \\
\hline lee-- & $-\cdots$ & .0004127 & .0002004 & .0000641 & -...- & .0001660 & .0000803 & .0000256 & $\ldots$ & .0000832 & .0000402 & .0000128 \\
\hline aaBbddEe-- & -..-- & .0004127 & .0002004 & & & .0001660 & & & & .0000832 & .0000402 & .0000128 \\
\hline aabbDdEe-- & & .0004127 & .0002004 & .0000641 & & .0001660 & .0000803 & .0000256 & -- & .0000832 & .0000402 & .0000128 \\
\hline &.- & 4126 & & & & & & & & & 203 & \\
\hline & -... & .0004126 & .0004976 & .0004441 & - & .0001660 & & .0001782 & & .0000832 & .0001203 & \\
\hline aabbDdee.- & 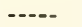 & .0004126 & .0005976 & .0004441 & & .0001660 & .0002403 & .0001782 & & .0000832 & .0001203 & .0000892 \\
\hline aabbddEe-- & & .0004126 & .0005976 & .0004441 & --- & .0001660 & .0002403 & .0001782 & & .0000832 & .0001203 & .0000892 \\
\hline aabbddee--. & 8745 & .9937846 & .9961105 & .9977992 & .9960119 & .9975056 & .9984402 & .9991182 & .9980030 & .9987514 & .9992194 & .9995588 \\
\hline
\end{tabular}


Tasle 39.--Relative frequeacies of eale genotypes when native population ( 3 autosumal genes BBODEE plus 1 sex-linked gene AA; female is heteroguetic) is overflooded by $\underline{x}$ release insects (abbbddee, a-bbddee) for every native insect

\begin{tabular}{|c|c|c|c|c|c|c|c|c|c|c|c|c|}
\hline \multirow{2}{*}{ Genotype } & & $\mathrm{x}$ & $=1$ & & & $\underline{x}$ & 2 & & & $\underline{x}$ & 3 & \\
\hline & $\overline{E_{1}}$ & $E=$ & 53 & $F_{i}$ & $F_{1}$ & $F_{2}$ & $\mathrm{~F}_{3}$ & $F_{4}$ & $F_{1}$ & $F_{2}$ & $\mathrm{~F}_{3}$ & $F_{4}$ \\
\hline$A A B B D D E=0.1$ & $1: 50000$ & 0.0095877 & 0.0003162 & 0.0000060 & 0.0555555 & 0.0017715 & 0.0000277 & 0.0000003 & 0.0312500 & 0.0005908 & 0,0000065 & 0.0000001 \\
\hline A133DDEC.- & $\ldots$ & .0021973 & .0003208 & .0000142 & -.... & .0007086 & .0000421 & .0000011 & ...... & .0003223 & .0000126 & .0000003 \\
\hline MS $2 D C E E$.. & -.... & .0021973 & .0003008 & $.00001+2$ & ..... & .0007086 & .0000421 & .0000011 & ..... & .0003223 & .0000126 & .0000003 \\
\hline MAS DOES .. & -..... & .0021973 & .0003005 & $.00001 * 2$ & -.... & .0007086 & .0000421 & .0000011 & ..... & .0003223 & .0000126 & .0000003 \\
\hline A. $33005 E \ldots$ & $\ldots$ & $.00 \geq 1973$ & .0003008 & $.00001+2$ & -...- & .0007086 & .0000421 & .0000011 & $\ldots$ & .0003223 & .0000126 & .0000003 \\
\hline MSBDDEC.. & -... & .0001221 & .0000716 & .000008 & $\ldots$ & .0000709 & .0000160 & .0000010 & -.... & .0000439 & .0000061 & .0000003 \\
\hline MSBddES.. & $-\ldots$ & .0001221 & .0000716 & .0000085 & $\ldots$ & .0000709 & .0000160 & .0000010 & ..... & .0000439 & .0000061 & .0000003 \\
\hline AA $b D D E Z=-$ & ...... & .0001221 & .0000716 & .000008 & $=\ldots$ & .0000709 & .0000160 & .0000010 & -.... & .0000439 & .0000061 & .0000003 \\
\hline AL\$3DDEE .. & $-\ldots$ & .0001221 & .0000716 & .0000084 & -...- & .0000709 & .0000160 & .0000010 & $-\ldots$ & .0000439 & .0000061 & .0000003 \\
\hline AABSDCEE - - & $-\ldots$ & $.002 \omega 010$ & .0006808 & $.0000 \div 41$ & $+\ldots$ & .0008503 & .0000932 & .0000052 & $-\ldots$ & .0004102 & .0000334 & .0000015 \\
\hline MJDDDEe -. & $\ldots$ & $.002 \div 0-1=$ & .0004308 & .0000441 & $-\ldots$ & .0008503 & .0000932 & .0000052 & $\ldots$ & .0004102 & .0000334 & .0000015 \\
\hline MBDDdEE -- & -.... & $.002-14$ & .0004508 & $.00004+1$ & -.-.- & .0008503 & .0000932 & .0000052 & ..... & .0004102 & .0000334 & .0000015 \\
\hline AM 33DDEe.. & -.... & $.002 \div-14$ & .0004808 & $.00004 i 1$ & ..... & .0008503 & .0000932 & .0000052 & -.... & .0004102 & .0000334 & .0000015 \\
\hline AaBSDdEE -- & $\ldots$ & $.002 \operatorname{lom} 16$ & .0006808 & $.0000<1$ & -.... & .0008503 & .0000932 & .0000052 & & .0004102 & .0000334 & .0000015 \\
\hline AaBb DOEE -. & $\ldots$ & $.002+-14$ & .0004308 & $.0000<61$ & $\ldots$ & .0008503 & .0000932 & .0000052 & --- & .0004102 & .0000334 & .0000015 \\
\hline Aus DDdee... & $\ldots$ & .000241 & .0001606 & .0000323 & ...- & .0001417 & .0000466 & .0000057 & $-\cdots$ & .0000879 & .0000205 & .0000020 \\
\hline dEe .- & $\ldots$ & $.00024-1$ & .0001606 & .0000323 & -... & .0001417 & .0000466 & .0000 & & .0000879 & .0000205 & .0000020 \\
\hline Mas Dore -. & $\ldots$ & .0002401 & .0001606 & .0000323 & $\ldots$ & .0001417 & .0000466 & .0000057 & -.... & .0000879 & .0000205 & .0000020 \\
\hline 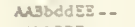 & $-\cdots-$ & $.0002 m 1$ & .0001606 & .0000323 & -.- & .0001417 & .0000466 & .0000057 & -..- & .0000879 & .0000205 & .0000020 \\
\hline MU BDDEE - - & $\ldots$ & .0002441 & .0001606 & .0000323 & -...- & .0001417 & .0000466 & .0000057 & -.... & .0000879 & .0000205 & .0000020 \\
\hline Mbs $x \mathrm{xt}$.. & $\ldots$ & $.0002 \div-1$ & .0001606 & .0000323 & ..... & .0001417 & .0000466 & .0000057 & & .0000879 & .0000205 & .0000020 \\
\hline ABDDeC - - & -... & $.0003 \div 1$ & .0001606 & .0000323 & $-\ldots$ & .0001417 & .0000466 & .0000057 & $-\ldots$ & .0000879 & .0000205 & .0000020 \\
\hline Aas3ddEZ -- & $-\ldots$ & $.0002+41$ & .0001606 & .0000323 & - - & .0001417 & .0000466 & .0000057 & ...... & .0000879 & .0000205 & .0000020 \\
\hline AABDDOEZ - - & $-\ldots$ & .0002441 & .0001606 & .0000323 & $-\ldots$ & .0001417 & .0000466 & .0000057 & & .0000879 & .0000205 & .0000020 \\
\hline as 3300Re -- & -... & .0002461 & .0001606 & .0000323 & $-\ldots$ & .0001417 & .0000466 & .0000057 & ..... & .0000879 & .0000205 & .0000020 \\
\hline A ABDdES .. & ..... & $.0002 \omega 1$ & .0001606 & .0000323 & $-\ldots-$ & .0001417 & .0000466 & .0000057 & -... & .0000879 & .0000205 & .0000020 \\
\hline AaBb DDEE -. & $\ldots$ & $.0002<-1$ & .0001606 & .0000323 & -.... & .0001417 & .0000466 & .0000057 & -...- & .0000879 & .0000205 & .0000020 \\
\hline MABbDCEe -- & ...... & .0029297 & .0010776 & .0001769 & $-\ldots$ & .0011338 & .0002703 & .0000292 & $\ldots .$. & .0005859 & .0001112 & .0000098 \\
\hline dEe -- & $\ldots$ & .0029297 & .0010774 & .0001769 & -.... & .0011338 & .0002703 & .000 & -.... & .0005859 & .0001 & .0000098 \\
\hline AasbDoce -. & $\ldots$ & .0029297 & .0010774 & .0001769 & $-\ldots$ & .0011338 & .0002703 & .0000 & $\ldots .$. & .0005859 & .0001112 & .0000098 \\
\hline A-ABDDEZ -. & $\ldots$ & .0029297 & .0010774 & .0001769 & $-\ldots$ & .0011338 & .0002703 & .0000292 & 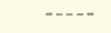 & .0005859 & .0001112 & .0000098 \\
\hline MBsBdzer... & $\ldots$. & .0001221 & .0000902 & .0000309 & -...- & .0000709 & .0000339 & .0000077 & -...- & .0000439 & .0000173 & .0000032 \\
\hline Dee =- & $-\ldots$ & .0001221 & .0000902 & .0000309 & -.... & .0000709 & .0000339 & .000 & $\ldots$ & .0000439 & .0000 & .0000032 \\
\hline buddER -- & ..... & .0001221 & .0000902 & .0000309 & $-\ldots$. & .0000709 & .0000339 & .0000077 & -... & .0000439 & .0000173 & .0000032 \\
\hline a a 38 Doee .- & $=\ldots$ & .0001221 & .0000902 & .0000309 & -...- & .0000709 & .0000339 & .0000077 & $-\ldots$ & .0000439 & .0000173 & .0000032 \\
\hline dEE -- & $-\ldots$ & .0001221 & .0000902 & .0000309 & ..... & .0000709 & .0000339 & .0000077 & -.... & .0000439 & .0000173 & .0000032 \\
\hline$\triangle A B b D D E E$-- & $\ldots$ & .0001221 & .0000902 & .0000309 & $\ldots$ & .0000709 & .0000339 & .0000077 & $-\ldots$ & .0000439 & .0000173 & .0000032 \\
\hline MBb DCee... & $\ldots \ldots$ & .0004883 & .0004636 & .0001568 & -... & .0002834 & .00016 & .00 & -.... & .00017 & .0000 & .000 \\
\hline M3bdcZe -. & $\ldots$ & .0004883 & .0004636 & .0001568 & -.... & .0002834 & .0001671 & .00 & ..... & .0001758 & .0000 & .0000153 \\
\hline MABDDCRE -. & $\ldots$ & .0004883 & .0004536 & .0001568 & -.... & .0002834 & .0001671 & .00 & -.... & .0001758 & .0000825 & .0000153 \\
\hline hasBDCee... & -..... & .0004883 & .0004636 & .0001568 & -..... & .0002834 & .0001671 & .0000376 & $\ldots$ & .0001758 & .0000825 & .0000153 \\
\hline dasaddRe -. & ...... & .0004883 & .0004636 & .0001568 & -..... & .0002834 & .0001671 & .00 & .... & .0001758 & .0000 & .0000153 \\
\hline Dee -- & -..-- & .0004883 & .0004636 & .0001568 & $-\ldots$ & .0002834 & .0001671 & .0000376 & $\ldots$ & .0001768 & .0000 & .0000153 \\
\hline ABbddzz -- & -.-- & .0004883 & .0004636 & .0001568 & $-\ldots$ & .0002834 & .0001671 & .0000376 & $-\ldots$ & .0001768 & .0000825 & .0000153 \\
\hline AabbDDEe -- & $\ldots$ & .0004883 & .0004636 & .0001568 & -... & .0002834 & .0001671 & .0000376 & ...- & .0001768 & .0000 & .0000153 \\
\hline dEZ .. & $\ldots .$. & .0006883 & .0004636 & .0001568 & $\ldots$ & .0002834 & .0001671 & .00 & -.... & .0001768 & .0000825 & .0000153 \\
\hline BBDARE -- & -..- & .0004883 & .0004636 & .0001568 & -.... & .0002834 & .0001671 & .0000376 & $\ldots$ & .0001768 & .0000825 & .0000153 \\
\hline a aBboDRe -. & -...- & .0004883 & .0004636 & .0001568 & -...- & .0002834 & .0001671 & .0000 & -.... & .0001768 & .0000825 & .0000153 \\
\hline 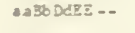 & $\ldots$ & .0004883 & .0004636 & .0001568 & ...... & .0002834 & .0001671 & .0000376 & $\ldots$ & .0001768 & .0000825 & .0000153 \\
\hline AdBbddee.. & -...- & .0002441 & .000 & & -.... & & & & -.... & & & \\
\hline MubbDdee -- & $+\ldots$ & .0002441 & .0003180 & .00018 & --- & .0001417 & .000 & .00 & ---- & .0000879 & .000 & .000 \\
\hline MubbddDe .. & ..... & .0002441 & .0003180 & .00018 & -... & .000 & .0001446 & .00 & -.... & .0000879 & .000 & .000 \\
\hline Idee -- & $\ldots .$. & .0002441 & .0003180 & .0001819 & $\ldots$ & .0001417 & .0001446 & .00 & -.... & .0000879 & .0000 & .0000291 \\
\hline AabbDDee .. & ..... & .0002441 & .0003180 & .0001819 & $-\cdots$ & .0001417 & .0001446 & .00 & -..- & .0000879 & .000 & .000 \\
\hline Aabboddzz -- & $\ldots$ & .0002641 & .0003180 & .000 & $\ldots$ & .001 & 1446 & & $\cdots$ & .0000 & 107 & .000 \\
\hline dee ... & -..- & .0002441 & .0003180 & .0001819 & -... & .000 & .000 & & -... & .0000879 & .0 & .0000291 \\
\hline dze -- & $\ldots$ & .0002441 & .0003180 & & -...- & .0001417 & 1446 & & -... & .0000879 & & \\
\hline Dee... & ..... & .0002441 & .0003180 & .0001819 & -.... & 1417 & 446 & & -.... & 879 & 807 & .0000291 \\
\hline ¿ABbddEZ -- & ..... & .0002441 & .0003180 & .0001819 & -..... & .0001417 & .0001446 & .00 & $\ldots . .$. & .0000879 & .0000807 & .0000291 \\
\hline abbDDEe -- & -....- & .0002441 & .0003180 & & - & .0001417 & & & -...- & & & .000 \\
\hline $2 a b b D C B E--$ & -..- & .0002441 & .0003180 & .0001819 & -...- & .0001417 & .0001446 & .0000603 & $\ldots$. & .0000879 & .0000807 & .0000291 \\
\hline Ze - . & 2500000 & .0917969 & .0205769 & .003475 & 2222222 & .04 & & & 1875000 & .02 & & \\
\hline dee-- & ..... & .0001221 & .0002804 & .0002679 & -... & .000 & .000 & & -...- & .000 & .000 & .000 \\
\hline Baddee -- & ....- & .0001221 & .0002804 & .0002679 & ...... & .000 & .0001542 & & ..... & 439 & 941 & .000 \\
\hline bDDee -- & --- & .0001221 & .000 & .00026 & $\ldots$ & & & & $-\ldots$ & & & .000 \\
\hline abbdddzz -- & -...- & .0001221 & .0002804 & .0002679 & -...- & .0000709 & .0001542 & .0001180 & -... & .0000439 & .0000941 & .0000655 \\
\hline ee -- & -.... & & & & -.... & & & & -.... & & & \\
\hline bddze -- & -... & .0107622 & .0099466 & 年 & -...- & 6372 & .005 & & $-\cdots$ & .0080859 & .0033552 & .0008131 \\
\hline bDdRe... & -.... & 107422 & .0099466 & .0039949 & ..... & & & & - & & 3552 & .0008131 \\
\hline aa36DdZe -- & $\ldots .$. & .0107422 & .0099466 & .0039949 & $\ldots$ & .0096372 & .0052419 & .0014921 & -.... & .0080859 & .0033552 & .0008131 \\
\hline lee -- & -..- & 0102539 & 46 & & -..- & & & & $-\ldots$ & & & \\
\hline & -..-- & .0102539 & .0106546 & .0071396 & 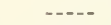 & .0093537 & .0073097 & .0037999 & -.... & .0079102 & .005 & .0025077 \\
\hline Aabbddze -- & -.... & .0102539 & .0106546 & .0071396 & $-\ldots$ & .0093537 & .0073097 & & -.... & .0079102 & .0054 & .0025077 \\
\hline Ddee -- & $\cdots$ & .0102539 & .0106546 & .0071396 & $-\ldots$ & .0093537 & .0073097 & &.-- & .0079102 & .0054397 & .0025077 \\
\hline aabbedze -. & ---- & .0102539 & .0106546 & .0071396 & ---- & .0093537 & .0073097 & .0037999 & $\cdots$ & .0079102 & .0054397 & .0025077 \\
\hline aabbDCEe -- & $\ldots$ & .0102539 & .0106546 & .0071396 & $\ldots$ & .0093537 & .0073097 & .0037999 & -..... & .0079102 & .0054397 & .0025077 \\
\hline ddee -. & -... & & .017 & & -....... & .00 & .014 & & $-\cdots$ & & & .0107095 \\
\hline Boddee -- & -..- & .0100098 & .0178170 & & $-\cdots$ & .0092120 & .0169304 & & & 078223 & .012 & \\
\hline aabbDdee -- & -... & .0100098 & .0178170 & .0194526 & -...- & .0092120 & .0149304 & .0139573 & -... & .0078223 & .0122382 & .0107095 \\
\hline aabbddze -- & $\ldots$ & .0100098 & .0178170 & .0194526 & -... & .0092120 & .0149304 & .0139573 & $\cdots$ & .0078223 & .0122382 & .0107095 \\
\hline aabbddee - - & 250000 & .2052002 & .2825332 & .3531170 & .2222222 & .2993906 & .3615028 & .4126291 & 2812500 & .3480907 & .3980546 & .4375997 \\
\hline
\end{tabular}


Table 39.--Relative frequencies of male genotypes when native population ( 3 autosomal genes BBDDEE plus 1 sex-1inked gene AA; female is heterogametic) is overflooded by $\underline{X}$ release insects (aabbddee, a-bbddee) for every native insect--Continued

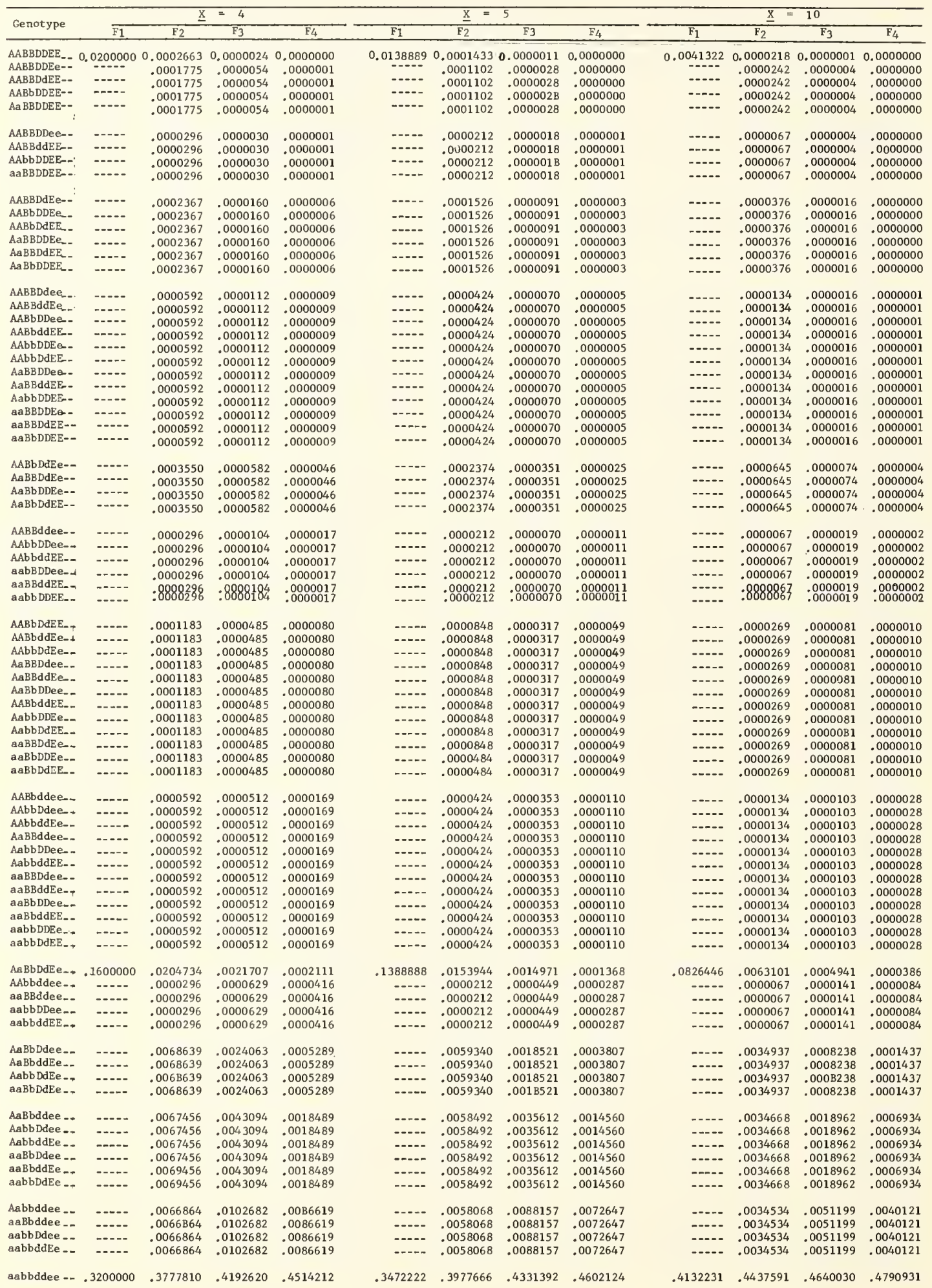


Table 39... Relasive frequencies of male genotypes when native population (3 autosonal genes. BBDDEE plus 1 sex-linked gene AA; female is heterogatic) is overflooded by $x$ release insects (aabbddee, a -bbddee) for every native insect.-Continued

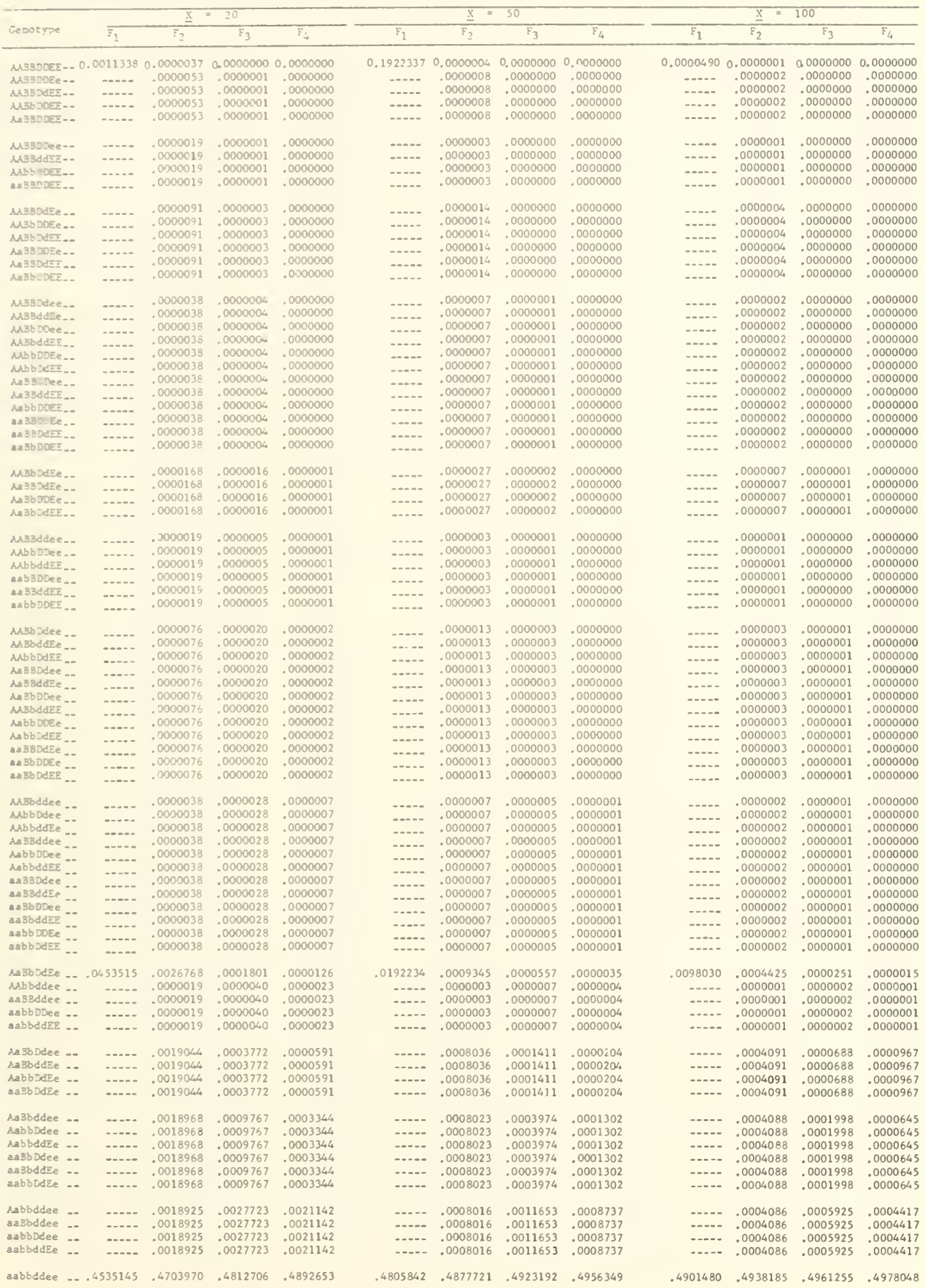


Table 39.--Relative frequencies of male genotypes when native population (3 autosomal genes BBDDEE plus 1 sex-linked gene AA; female is heterogametic) is overflooded by $\underline{X}$ release insects (abbddee, a-bbddee) for every native insect.--Continued

\begin{tabular}{|c|c|c|c|c|c|c|c|c|c|c|c|c|c|}
\hline & & & $\underline{x}=$ & 200 & & & $\underline{x}=$ & 500 & & & $\underline{x}$ & 1,000 & \\
\hline & & $\mathrm{F}_{1}$ & $\mathrm{~F}_{2}$ & $\mathrm{~F}_{3}$ & $\mathrm{~F}_{4}$ & $F_{1}$ & $\mathrm{~F}_{2}$ & $\mathrm{~F}_{3}$ & $\mathrm{~F}_{4}$ & $F_{1}$ & $F_{2}$ & $\mathrm{~F}_{3}$ & $F_{4}$ \\
\hline $\mathrm{AABBDDEE}^{--}$ & & 0000124 & 0.0000000 & 0.0000000 & 0.0000000 & 0.0000020 & 0.0000000 & 0.0000000 & 0.0000000 & 0.0000005 & 0.0000000 & 0.0000000 & 0.0000000 \\
\hline AABBDDEe - & & --- & .0000000 & .0000000 & .0000000 & -... & .0000000 & .0000000 & .0000000 & ----- & .0000000 & .0000000 & .0000000 \\
\hline AABBDdEE - & & $-\infty$ & .0000000 & .0000000 & .0000000 & & .0000000 & .0000000 & .0000000 & & .0000000 & .0000000 & .0000000 \\
\hline AABbDDEE - & & $-\cdots$ & .0000000 & .0000000 & .0000000 & $-\cdots$ & .0000000 & .0000000 & .0000000 & -..-- & .0000000 & .0000000 & .0000000 \\
\hline AaBBDDEE - & & $\ldots$ & .0000000 & .0000000 & .0000000 & $-\cdots$ & .0000000 & .0000000 & .0000000 & ---- & .0000000 & .0000000 & .0000000 \\
\hline AABBDDee $=-$ & & & .0000000 & .0000000 & .0000000 & -..- & .0000000 & .0000000 & .0000000 & -.-- & .0000000 & .0000000 & .0000000 \\
\hline AABBddEE - & & --.-- & .0000000 & .0000000 & .0000000 & $=-1$ & .0000000 & .0000000 & .0000000 & & .0000000 & .0000000 & .0000000 \\
\hline AAb bDDEE $\rightarrow$ & & --- & .0000000 & .0000000 & .0000000 & ---- & .0000000 & .0000000 & .0000000 & --- & .0000000 & .0000000 & .0000000 \\
\hline$a_{a B B D D E E}-$ & & $-\cdots$ & .0000000 & .0000000 & .0000000 & -..- & .0000000 & .0000000 & .0000000 & $\cdots$ & .0000000 & .0000000 & .0000000 \\
\hline AABBDdEe - & & $\cdots-$ & .0000001 & .0000000 & .0000000 & 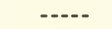 & .0000000 & .0000000 & .0000000 & $-\ldots$ & .0000000 & .0000000 & .0000000 \\
\hline AABb DDEe - & & $\ldots$ & .0000001 & .0000000 & .0000000 & & .0000000 & .0000000 & .0000000 & - & .0000000 & .0000000 & .0000000 \\
\hline AABb DdEE - & & - & .0000001 & .0000000 & .0000000 & -..... & .0000000 & .0000000 & .0000000 & & .0000000 & .0000000 & .0000000 \\
\hline $\mathrm{AaBBDDEe}$ & & $\ldots$ & .0000001 & .0000000 & .0000000 & $\ldots$ & .0000000 & .0000000 & .0000000 & --- & .0000000 & .0000000 & .0000000 \\
\hline AaBBDdEE - & & $\ldots$ & .0000001 & .0000000 & .0000000 & $-\ldots$ & .0000000 & .0000000 & .0000000 & & .0000000 & .0000000 & .0000000 \\
\hline AaBbDDEE - & & ..... & .0000001 & .0000000 & .0000000 & $-\cdots$ & .0000000 & .0000000 & .0000000 & - - & .0000000 & .0000000 & .0000000 \\
\hline AABBDdee - & & -- & .0000000 & .0000000 & .0000000 & $-\ldots$ & .0000000 & .0000000 & .0000000 & -... & .0000000 & .0000000 & .0000000 \\
\hline AABBddEe - & & -- & .0000000 & .0000000 & .0000000 & $-\ldots$ & .0000000 & .0000000 & .0000000 & $-\cdots$ & .0000000 & .0000000 & .0000000 \\
\hline AABbDDee - & & -... & .0000000 & .0000000 & .0000000 & -... & .0000000 & .0000000 & .0000000 & ----- & .0000000 & .0000000 & .0000000 \\
\hline AABbddEE - & & ..... & .0000000 & .0000000 & .0000000 & -.... & .0000000 & .0000000 & .0000000 & $-\cdots$ & .0000000 & .0000000 & .0000000 \\
\hline AABb DDEe - & & $\ldots$ & .0000000 & .0000000 & .0000000 & -...- & .0000000 & .0000000 & .0000000 & $\ldots$ & .0000000 & .0000000 & .0000000 \\
\hline AAbbDdEE - & & -... & .0000000 & .0000000 & .0000000 & -...- & .0000000 & .0000000 & .0000000 & --- & .0000000 & .0000000 & .0000000 \\
\hline AaBBDDee - & & -... & .0000000 & .0000000 & .0000000 & -... & .0000000 & .0000000 & .0000000 & --- & .0000000 & .0000000 & .0000000 \\
\hline AaB BddEE - & & ...... & .0000000 & .0000000 & .0000000 & $-\ldots$ & .0000000 & .0000000 & .0000000 & $-\cdots$ & .0000000 & .0000000 & .0000000 \\
\hline Aabb DDEE - & & -..- & .0000000 & .0000000 & .0000000 & -.... & .0000000 & .0000000 & .0000000 & -.... & .0000000 & .0000000 & .0000000 \\
\hline a $a$ BBDDEe - & & $\ldots$ & .0000000 & .0000000 & .0000000 & -...- & .0000000 & .0000000 & .0000000 & -..- & .0000000 & .0000000 & .0000000 \\
\hline a aBBDdEE - & & -... & .0000000 & .0000000 & .0000000 & -...- & .0000000 & .0000000 & .0000000 &.--- & .0000000 & .0000000 & .0000000 \\
\hline $\mathrm{a} a B b \mathrm{DDEE}$ - & & ..... & .0000000 & .0000000 & .0000000 & $-\ldots$ & .0000000 & .0000000 & .0000000 & $=-$ & .0000000 & .0000000 & .0000000 \\
\hline AABbDdEe - & & --- & .0000002 & .0000000 & .0000000 & $-\cdots$ & .0000000 & .0000000 & .0000000 & & .0000000 & .0000000 & .0000000 \\
\hline AaBBDdEe - & & $\ldots$ & .0000002 & .0000000 & .0000000 & ----- & .0000000 & .0000000 & .0000000 & ----- & .0000000 & .0000000 & .0000000 \\
\hline $\mathrm{AaBbDDEe}$ - & & -... & .0000002 & .0000000 & .0000000 & -...- & .0000000 & .0000000 & .0000000 & ---- & .0000000 & .0000000 & .0000000 \\
\hline $\mathrm{AaBbDdEE}$ - & & --- & .0000002 & .0000000 & .0000000 & 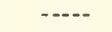 & .0000000 & .0000000 & .0000000 & ---- & .0000000 & .0000000 & .0000000 \\
\hline AABBddee - & & ..... & .0000000 & .0000000 & .0000000 & -..- & .0000000 & .0000000 & .0000000 & --.-- & .0000000 & .0000000 & .0000000 \\
\hline AAbbDDee - & & - & .0000000 & .0000000 & .0000000 & -..- & .0000000 & .0000000 & .0000000 & & .0000000 & .0000000 & .0000000 \\
\hline AAbbddEE - & & -- & .0000000 & .0000000 & .0000000 & -.... & .0000000 & .0000000 & .0000000 & ---- & .0000000 & .0000000 & .0000000 \\
\hline aabBDDee - & &.-- & .0000000 & .0000000 & .0000000 & -... & .0000000 & .0000000 & .0000000 & & .0000000 & .0000000 & .0000000 \\
\hline aaBBddEE - & & $\ldots$ & .0000000 & .0000000 & .0000000 & & .0000000 & .0000000 & .0000000 & & .0000000 & .0000000 & .0000000 \\
\hline aabbDDEE - & & ..... & .0000000 & .0000000 & .0000000 & -.... & .0000000 & .0000000 & .0000000 & 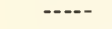 & .0000000 & .0000000 & .0000000 \\
\hline AABbDdee - & & $\ldots$ & .0000001 & .0000000 & .0000000 & $\ldots$ & .0000000 & .0000000 & .0000000 & $--\cdots$ & .0000000 & .0000000 & .0000000 \\
\hline AABbddEe . & & $\ldots$ & .0000001 & .0000000 & .0000000 & & .0000000 & .0000000 & .0000000 & & .0000000 & .0000000 & .0000000 \\
\hline AAbbDdEe - & & $\ldots$ & .0000001 & .0000000 & .0000000 & & .0000000 & .0000000 & .0000000 & 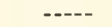 & .0000000 & .0000000 & .0000000 \\
\hline AaBBDdee - & & --- & .0000001 & .0000000 & .0000000 &.-- & .0000000 & .0000000 & .0000000 & -.--- & .0000000 & .0000000 & .0000000 \\
\hline AaBBddEe - & & $\ldots$ & .0000001 & .0000000 & .0000000 & ... & .0000000 & .0000000 & .0000000 & & .0000000 & .0000000 & .0000000 \\
\hline AaBbDDee - & & -- & .0000001 & .0000000 & .0000000 & $\cdots$ & .0000000 & .0000000 & .0000000 & $\cdots$ & .0000000 & .0000000 & .0000000 \\
\hline AABbddEE - & & $\ldots$ & .0000001 & .0000000 & .0000000 & $\ldots$ & .0000000 & .0000000 & .0000000 & -..- & .0000000 & .0000000 & .0000000 \\
\hline AabbDDEe - & & -.... & .0000001 & .0000000 & .0000000 & -... & .0000000 & .0000000 & .0000000 & ---- & .0000000 & .0000000 & .0000000 \\
\hline AabbDdEE - & & -...- & .0000001 & .0000000 & .0000000 & --- & .0000000 & .0000000 & .0000000 & --.-- & .0000000 & .0000000 & .0000000 \\
\hline aaBBDdEe - & & $\ldots$ & .0000001 & .0000000 & .0000000 & $\ldots-$ & .0000000 & .0000000 & .0000000 & & .0000000 & .0000000 & .0000000 \\
\hline aaBbDDEe - & & -... & .0000001 & .0000000 & .0000000 & -.... & .0000000 & .0000000 & .0000000 & $-\cdots$ & .0000000 & .0000000 & .0000000 \\
\hline aaBbDdEE _ & & -..-- & .0000001 & .0000001 & .0000000 & --.- & .0000000 & .0000000 & .0000000 & --- & .0000000 & .0000000 & .0000000 \\
\hline AABbddee - & & $\ldots$ & .0000000 & .0000000 & .0000000 & - - & .0000000 & .0000000 & .0000000 & $-\cdots$ & .0000000 & .0000000 & .0000000 \\
\hline AAbb Ddee - & & ..... & .0000000 & .0000000 & .0000000 & -... & .0000000 & .0000000 & .0000000 & & .0000000 & .0000000 & .0000000 \\
\hline AAbbddEe - & & $-\ldots$ & .0000000 & .0000000 & .0000000 & $-\ldots$ & .0000000 & .0000000 & .0000000 & -..- & .0000000 & .0000000 & .0000000 \\
\hline AaBBddee. & & $\ldots$ & .0000000 & .0000000 & .0000000 & $\ldots$ & .0000000 & .0000000 & .0000000 & & .0000000 & .0000000 & .0000000 \\
\hline AabbDDee - & & $=-$ & .0000000 & .0000000 & .0000000 & -...- & .0000000 & .0000000 & .0000000 & $\cdots$ & .0000000 & .0000000 & .0000000 \\
\hline AabbddEE - & & -.... & .0000000 & .0000000 & .0000000 & -..- & .0000000 & .0000000 & .0000000 & --.- & .0000000 & .0000000 & .0000000 \\
\hline aaBBDdee - & - & -... & .0000000 & .0000000 & .0000000 & $\cdots-$ & .0000000 & .0000000 & .0000000 & $-+-\infty$ & .0000000 & .0000000 & .0000000 \\
\hline aaBBddEe - & -- & -.... & .0000000 & .0000000 & .0000000 & -.-- & .0000000 & .0000000 & .0000000 & - n. & .0000000 & .0000000 & .0000000 \\
\hline aaBbDDee - & - & -... & .0000000 & .0000000 & .0000000 & $-\cdots-$ & .0000000 & .0000000 & .0000000 & $-\ldots$ & .6000000 & .0000000 & .0000000 \\
\hline aaBbddEE - & -- & -.... & .0000000 & .0000000 & .0000000 & $-\cdots$ & .0000000 & .0000000 & .0000000 & -...- & .0000000 & .0000000 & .0000000 \\
\hline a abbDDEe - & - &..-- & .0000000 & .0000000 & .0000000 & -.... & .0000000 & .0000000 & .0000000 & ----- & .0000000 & .0000000 & .0000000 \\
\hline aAbbDdEE - & -- & $-\cdots$ & .0000000 & .0000000 & .0000000 & $-\ldots$ & .0000000 & .0000000 & .0000000 & $\cdots$ & .0000000 & .0000000 & .0000000 \\
\hline $\mathrm{AaBbDdEe}$ & $\ldots$ & 0049504 & .0002149 & .0000119 & .0000007 & .0019920 & .0000844 & .0000046 & .0000003 & .0009980 & .0000419 & .0000023 & .0000001 \\
\hline AAbbddee - & - & -...- & .0000000 & .0000000 & .0000000 & --- & .0000000 & .0000000 & .0000000 & -.... & .0000000 & .0000000 & .0000000 \\
\hline aaBBddee & -- & -.... & .0000000 & .0000000 & .0000000 & ---- & .0000000 & .0000000 & .0000000 & -..-- & .0000000 & .0000000 & .0000000 \\
\hline aebbDDee - & -- & -...- & .0000000 & .0000000 & .0000000 & $-\cdots$ & .0000000 & .0000000 & .0000000 & --- & .0000000 & .0000000 & .0000000 \\
\hline aabbddEE & -- & --- & .0000000 & .0000000 & .0000000 & $\cdots$ & .0000000 & .0000000 & .0000000 & 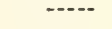 & .0000000 & .0000000 & .0000000 \\
\hline AaBbDdee - & -- & ---- & .0002064 & .0000339 & .0000047 & ---- & .0000830 & .0000135 & .000 & -..-- & .0000416 & .0000067 & 009 \\
\hline AaBbddEe - & -- & -.... & .0002064 & .0000339 & .0000047 & $-\cdots$ & .0000830 & .0000135 & .0000018 & --.-- & .0000416 & .0000067 & .0000009 \\
\hline AabbDdEe - & -- & - & .0002064 & .0000339 & .0000047 & $\ldots$ & .0000830 & .0000135 & .0000018 & --- & .0000416 & .0000067 & .0000009 \\
\hline aaBbDdEe & -- & -..- & .0002064 & .0000339 & .0000047 & $-\cdots$ & .0000830 & .0000135 & .0000018 & $-\ldots$ & .0000416 & .0000067 & .0000009 \\
\hline AaBbddee & -- & -..-- & .0002064 & .0001002 & .0000321 & -... & .0000830 & .0000401 & .0000129 & ---- & .0000419 & .0000201 & .0000064 \\
\hline AabbDdee & -- & --- & .0002064 & .0001002 & .0000321 & -...- & .0000830 & .0000401 & .0000129 & --- & .0000419 & .0000201 & .0000064 \\
\hline AabbddEe & -- & $\cdots$ & .0002064 & .0001002 & .0000321 & -.... & .0000830 & .0000401 & .0000129 & ---- & .0000419 & .0000201 & .0000064 \\
\hline aaBbDdee & -- & $\ldots$ & .0002064 & .0001002 & .0000321 & $-\ldots$ & $.0000 \mathrm{~B} 30$ & .0000401 & .0000129 & --.- & .0000419 & .0000201 & .0000064 \\
\hline aaBbddEe & -- & --- & .0002064 & .0001002 & .0000321 & $\ldots$ & .0000830 & .0000401 & .0000129 & --- & .0000419 & .0000201 & .0000064 \\
\hline aabbDdEe & -- & ---- & .0002064 & .0001002 & .0000321 & $\cdots$ & .0000830 & .0000401 & .0000129 & ---- & .0000419 & .0000201 & .0000064 \\
\hline Aabbddee & -- & $-\ldots$ & .0002063 & .0002988 & .0002221 & - & .0000830 & .0001201 & $.0000 B 91$ & --- & .0000416 & .0000602 & \\
\hline aaBbddee & -- & --..- & .0002063 & .0002988 & .0002221 & -..- & .0000830 & .0001201 & .0000891 & $-\cdots$ & .0000416 & .0000602 & .0000446 \\
\hline aabbDdee & -- & & .0002063 & .0002988 & .0002221 & --.-- & .0000830 & .0001201 & .0000891 & $\cdots$ & .0000416 & .0000602 & .0000446 \\
\hline aabbddEe & -- &.--- & .0002063 & .0002988 & .0002221 & $-\ldots$ & .0000830 & .0001201 & $.0000 \mathrm{~B} 91$ & ---- & .0000416 & .0000602 & .0000446 \\
\hline aabbddee & & 50372 & .4968929 & .4980550 & .4988995 & .4980059 & .4987526 & .4992195 & .4995586 & .4990015 & .4993756 & .4996095 & .4997792 \\
\hline
\end{tabular}


able $40 .-$ Relative frecuencies of female genotypes when native population ( 3 autosomal genes BBDDEE plus 1 sex-linked gene AA; temale is heterogametic) is overflooded by $x$ release insects (abbddee, a-bbddee) for every native insect

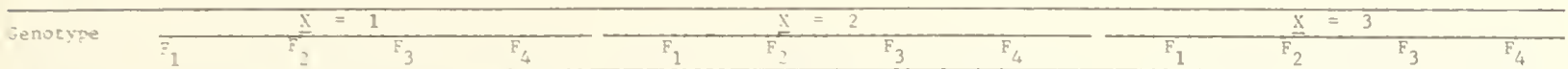

$4=-0.1250020 .-109503 \quad 0.0760600 .0700131 \quad 0.0555: 53 \quad 0.0021258 \quad 0.00004870 .0000009$

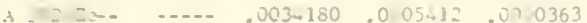

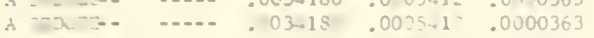

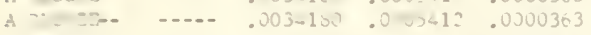

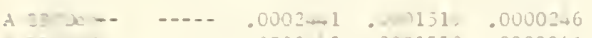

A. 20.-. $0.0002-1 \quad .0001519 \quad .0000246$

A S2DE- - .... .0002441 .000151- .0000246

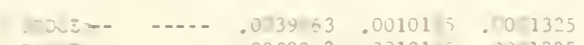

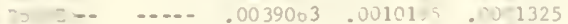

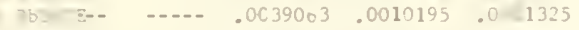

$A=x \ldots 0 . . .0 .0004383 \quad .0003924 .00110 \mathrm{~b}$

A BBCdEC-

A sbonee- ..... .004583:003924:001111

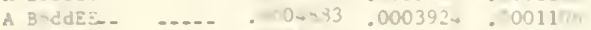

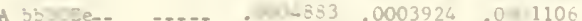

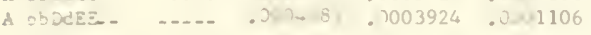

a BBDDEE- ..... .01220, .002221 .000155

A. $10 . .125000 .0488281 \quad .7123659,019150$

BBDFEA- -.... .001464* .0004010 .0000543

a BBDAES-

a $3-0.55-1 . . .0014648,0004010,0000543$

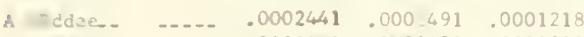

A BDD-e. - .-.. .0002441 .0402491 .0001218

A HedzE- ..... .0 2641 .0002491.001218

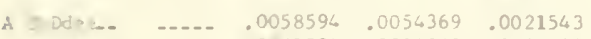

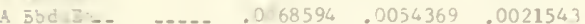

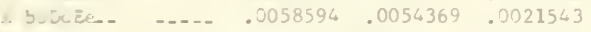

S JED DeE-

I C CdEL.

$\begin{array}{cccc}0.0 .0 & .0002441 & .0001706 & .0000470\end{array}$

A

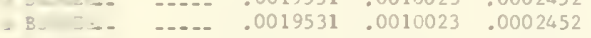

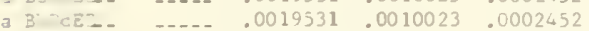

$\begin{array}{llllll}1.0 . & 0.053711 & .0055453 & .0037518\end{array}$

$1=0.0 .0 .0053711 \quad .0056453 \quad .0037518$

4. $=0053711.0056453 .0037518$

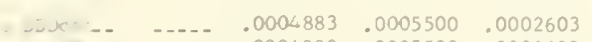

$\begin{array}{lllll}32 d c \ldots & .0004883 & .0005500 & .0002603\end{array}$

$.0004883-0005500$

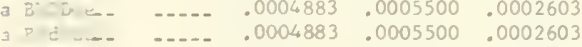

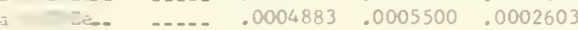

$\begin{array}{llll}0.0 & 0004883 & .0005500 & .0002603\end{array}$

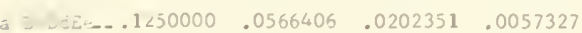

a Judies-

$\begin{array}{lllll} & 0.000 & 0002441 & .0004394 & .0003589\end{array}$

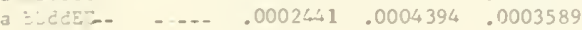

d.tids- $\quad \ldots . . .0051270 \quad .0091813 \quad .0099943$

$\begin{array}{lllll}\text { zoviog - } & 0155250 & .0156279 & .0091370\end{array}$

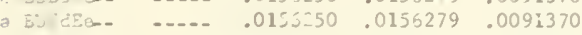

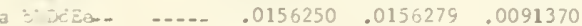

$\begin{array}{lllll}\text { a Rbedd_es } & \ldots . . . & 0151367 & 0231290 & .0230223\end{array}$

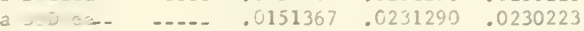

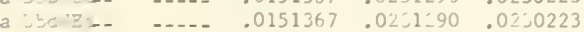

$\left[\begin{array}{llll}0.0011338 & .0000887 & .0000037\end{array}\right.$

$\begin{array}{llll}0.0 & .0011338 & .0000887 & .00000037 \\ \cdots & .0000037\end{array}$

$\left[\begin{array}{llll}0 .- & .0011338 & .0000887 & .000000037\end{array}\right.$

$\left[\begin{array}{llll}0.0 & 0001417 & .0000393 & .0000039\end{array}\right.$

$\left[\begin{array}{llll}0.0001 山 17 & .0000393 & .0000039\end{array}\right.$

[... $\quad .0001417 \quad .0000393 \quad .0000039$

$\begin{array}{llll}0 .-0 & .0014172 & .0002284 & .0000198\end{array}$

$\begin{array}{llll}-0 .-0 & .0014172 & .0002284 & .0000198\end{array}$

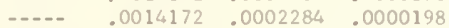

$\left[\begin{array}{cccc}0 .-0 & 0002834 & .0001301 & .0000244\end{array}\right.$

. $\quad .002834 \quad .0001301 \quad .0000244$

$\begin{array}{llll}0.0002834 & .0001301 & .0000244\end{array}$

$\begin{array}{llll}\ldots .0 & .0002834 & .0001301 & .0000244\end{array}$

$\begin{array}{lllll}0.0 & .0002834 & .0001301 & .0000244\end{array}$

[.- .0002834 $.0001301 \quad .0000244$

.... $\quad .0006252 \quad .0000370 \quad .0000016$

$\begin{array}{llll}.1111111 & .0246598 & .0037339 & .0004570\end{array}$

$\begin{array}{llll}0.0 & .0005669 & .0000932 & .0000083\end{array}$

$\begin{array}{llll}0.0 & .0005669 & .0000932 & .0000083 \\ \cdots & .0005669 & .0000932 & .0000083\end{array}$

$\begin{array}{llll}{[-.-} & .0005669 & .0000932 & .00000083\end{array}$

$\begin{array}{llll}-0.0001417 & .0001062 & .0000378\end{array}$

$\begin{array}{llll} & .0001417 & .0001065 & .0000378\end{array}$

$\begin{array}{llll}-0.0001417 & .0001062 & .0000378 \\ 0.0000378\end{array}$

$\begin{array}{llll}0.0051020 & .0027881 & .0007837\end{array}$

$.0051020 \quad .0027881$
0.0007837

$\begin{array}{llll}0.0 & 0001417 & .0000572 & .0000105\end{array}$

$\begin{array}{llll}0.0001417 & .0000572 & .0000105\end{array}$

$\begin{array}{llll}0 .- & .0001417 & .0000572 & .0000105 \\ \cdots & .0001417 & .0000572 & .0000105\end{array}$

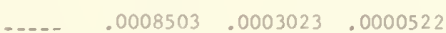

$\begin{array}{llll}0.0 & .0008503 & .0003023 & .0000522 \\ \cdots & .0008503 & .0003023 & .0000522\end{array}$

$\begin{array}{llll}.0008503 & .0003023 \quad .0000522\end{array}$

$\begin{array}{llll} & 0048186 & .0037995 & .0019603\end{array}$

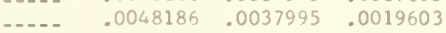

$\begin{array}{llll}0.0048186 & .0037995 & .0019603\end{array}$

$\begin{array}{lllll} & .0002834 & .0002281 & .0000791\end{array}$

$\begin{array}{lllll}0.0002834 & .0002281 & .0000791\end{array}$

$\begin{array}{llllll} & 0.0002834 & .0002281 & .0000791\end{array}$

$\begin{array}{lllll}0 . .- & .0002834 & .0002281 & .0000791\end{array}$

$\begin{array}{llll}\cdots & .0002834 & .0002281 & .0000791 \\ \cdots & .0002834 & .0002281 & .0000791\end{array}$

$\begin{array}{llll}.1111111 & .0331632 & .0087049 & .0019198\end{array}$

$\begin{array}{lllll}\ldots . . & .0001417 & .0002265 & .0001482\end{array}$

$\begin{array}{lllll} & .0001417 & .0002265 & .0001482 \\ 0.0 & .0001417 & .0002265 & .0001482\end{array}$

$\begin{array}{llll}\cdots & .0001417 & .0002265 & .0001482\end{array}$

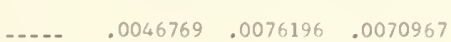

$\begin{array}{llll}0.0 & 0141723 & .0099302 & .0045459\end{array}$

$\left[\begin{array}{llll}{[.-0} & .0141723 & .009930 ? & .0045459\end{array}\right.$

$\left[\begin{array}{llll}\ldots & .0141723 & .0099302 & .0045459\end{array}\right.$

$\begin{array}{llll}-0 .-0138889 & .0185852 & .0158571\end{array}$

$\begin{array}{llll}0.0138889 & .0185852 & .0158571\end{array}$

$\begin{array}{lllll} & .0138889 & .0185852 & .0158571\end{array}$ $\begin{array}{lllll} & 0051020 & .0027881 & .0007837\end{array}$

$\begin{array}{llll}.0002834 & .0002281 & .0000791\end{array}$
$0.0312500 \quad 0.00075 ? 0 \quad 0.07001280 .0000002$

[-. $\quad .0005273 \quad .0000293 \quad .0000010$

$\begin{array}{lllll}0 .- & .0005273 & .0000293 & .0000010\end{array}$

.... $.0005273,0000293,0000010$

$\begin{array}{llll} & 0.0000879 & .0000164 & .0000013\end{array}$

$\begin{array}{llll}0.0 & .0000879 & .0000164 & .0000013\end{array}$

$\begin{array}{lllll}0 .- & 0000879 & 0000164 & .0000013\end{array}$

$\begin{array}{llll}\ldots . .0 & .0007031 & .0000890 & .0000064\end{array}$

... $0.0007031 \quad .0000890 \quad .0000064$

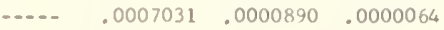

$\begin{array}{llll}0.0 & 0001758 & .0000618 & .0000096\end{array}$

$0.001758-0000618.0000096$

[.- $.0001758 \quad .0000618 \quad .0000096$

$\begin{array}{llll}0.0001758 & .0000618 & .0000096\end{array}$

$\begin{array}{lll}0001758 \quad 0000618 & 0000096\end{array}$

$\begin{array}{llll}\ldots . . & .0001758 & .0000618 & .00000096\end{array}$

.0002051 .0000124

.0000004

$\begin{array}{llll}.0937500 & 0152344 & .0018708 & .0001972\end{array}$

$\begin{array}{lllll} & .0002930 & .0000373 & .0000027\end{array}$

$\begin{array}{llll}0.0 & .0002930 & .0000373 & .0000027\end{array}$

$0002930 \quad 0000373.0000027$

$\begin{array}{lllll}0.0 & .0000879 & .0000577 & .0000178\end{array}$

$\begin{array}{lllll} & 0000879 & 0000577 & .0000178\end{array}$

$\begin{array}{llll}\cdots & .0000879 & .0000577 & .0000178\end{array}$

$\begin{array}{lllll} & .0042187 & .0017601 & .0004219\end{array}$

$0.0042187 .0017601-0004219$

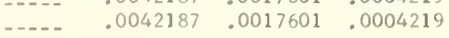

$\begin{array}{llll}.0000879 & .0000276 & .0000042\end{array}$

$\begin{array}{llll}0.0000879 & .0000276 & .000004 \text { ? }\end{array}$

$\begin{array}{llll}{[-. .} & 0000879 & .0000276 & .0000042\end{array}$

$\begin{array}{lllll} & & 0004687 & .0001381 & .0000202\end{array}$

$\begin{array}{llll} & .0004687 & .0001381 & .0000202\end{array}$

$\begin{array}{llll}\cdots & .0004687 & .0001381 & .0000202\end{array}$

$\begin{array}{llll} & .0040430 & .0028007 & .0012829\end{array}$

$\begin{array}{lllll}{[. .-} & .0040430 & .0028007 & .0012829\end{array}$

$\begin{array}{llll}0.0040430 & .0028007 & .0012829\end{array}$

$\begin{array}{llll}0.0001758 & .0001220 & .0000367\end{array}$

$0001758 \quad 0001220.0000367$

$\begin{array}{llll}\cdots \cdots & .0001758 & .0001220 & .0000367 \\ \cdots & .0001758 & .0001220 & .0000367\end{array}$

$\begin{array}{llll}{[.-} & .0001758 & .0001220 & .00000367 \\ \cdots & .0001758 & .0001220 & .0000367\end{array}$

$\begin{array}{llll}0.001758 & 0001220 & 0000367\end{array}$

$\begin{array}{lllll}0.0001758 & .0001220 & .0000367\end{array}$

$.0937500 \quad .0227344 \quad .0051142 \quad .0010004$

$\begin{array}{llll} & .0000880 & .0001344 & .0000801\end{array}$

$\left[\begin{array}{llll}0.0 & .0000880 & .0001344 & .0000801\end{array}\right.$

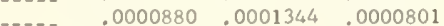

[... $\quad 0039551 \quad 0062132 \quad .0054203$

$\begin{array}{llll}0.0119531 & .0071173 & .0029142\end{array}$

$\begin{array}{lllll}0.0119531 & .0071173 & .0029142\end{array}$

$-0119531-0071173-0029142$

$\begin{array}{llll}0.0117773 & .0149581 & .0119632\end{array}$

$\begin{array}{llll}0.0117773 & .0149581 & .0119632\end{array}$

.0117773 $\quad .0149581 \quad .0119632$ 
Table 40.--Relative frequencies of female genotypes when native population ( 3 autosomal genes BBDDEE plus 1 sex-1inked gene $\mathrm{AA}$; female is heterogametic) is overflooded by $\underline{X}$ release insects (aabbddee, a-bbddee) for every native insect--
Continued

\begin{tabular}{|c|c|c|c|c|c|c|c|c|c|c|c|c|c|}
\hline \multirow{2}{*}{\multicolumn{2}{|c|}{ Genotype }} & \multicolumn{4}{|c|}{$\underline{x}=4$} & \multicolumn{4}{|c|}{$\underline{x}=5$} & \multicolumn{4}{|c|}{$\underline{x}=10$} \\
\hline & & $\mathrm{F}_{1}$ & $\mathrm{~F}_{2}$ & $\mathrm{~F}_{3}$ & $\mathrm{~F}_{4}$ & $\mathrm{~F}_{1}$ & $\mathrm{~F}_{2}$ & $\mathrm{~F}_{3}$ & $\mathrm{~F}_{4}$ & $\mathrm{~F}_{1}$ & $\mathrm{~F}_{2}$ & $F_{3}$ & $\mathrm{~F}_{4}$ \\
\hline A & BBDDEE -0 . & 200000 & 0.0003550 & 0.0000051 & 0.0000001 & 0.01400000 & 0.0001934 & 0.0000025 & 0.0000000 & 0.0041322 & 0.0000339 & 0.0000003 & 0.0000000 \\
\hline & BBDDEe -- & -.-- & $.000 \approx 959$ & .0000134 & .0000004 & & .0001865 & .0000073 & .0000002 & & .0000430 & .0000012 & .0000000 \\
\hline & BBDdEE -- & $-\cdots$ & .0002959 & .0000134 & .0000004 & & .0001865 & .0000073 & .0000002 & -..- & .0000430 & .0000012 & .0000000 \\
\hline & $\mathrm{BbDDEE}^{--}$ & --.- & .0002959 & .0000134 & .0000004 & -... & .0001865 & .0000073 & .0000002 & $\cdots$ & .0000430 & .0000012 & .0000000 \\
\hline & BBDDee -- & & .0000592 & .0000086 & .0000006 & & .0000424 & .0000053 & .0000003 & & .0000134 & .0000011 & .0000001 \\
\hline & BBddEE - - & $---\cdot$ & .0000592 & .0000086 & .0000006 & & .0000424 & .0000053 & .0000003 & & .0000134 & .0000011 & .0000001 \\
\hline & BbDDEE -- & -... & .0000592 & .0000086 & .0000006 & ----- & .0000424 & .0000053 & .0000003 & & .0000134 & .0000011 & .0000001 \\
\hline & BBDdEe _- & & .0004142 & .0000451 & .0000029 & & .0002713 & .0000266 & .0000016 & & .0000699 & .0000053 & .0000003 \\
\hline & BbDDEe _- & $\cdots$ &, 0004142 & .0000451 & .0000029 & & .0002713 & .0000266 & .0000016 & & .0000699 & .0000053 & .0000003 \\
\hline & BbDdEE .- & & .0004142 & .0000451 & .0000029 & & .0002713 & .0000266 & .0000016 & & .0000699 & .0000053 & .0000003 \\
\hline & BBDdEE _- & & .0001183 & .0000355 & .0000050 & & .0000848 & .0000228 & .0000030 & ---- & .0000269 & .0000057 & .0000006 \\
\hline & BBddEe-- & -.-- & .0001183 & .0000355 & .0000050 & & .0000848 & .0000228 & & -.... & .0000269 & .000 & \\
\hline & BbDDee-- & $-\ldots$ & .0001183 & .0000355 & .0000050 & ---- & .0000848 & .0000228 & .0000 & $\ldots$ & .0000269 & .0000057 & .0000006 \\
\hline & BbddEE-- & ---- & .0001183 & .0000355 & .0000050 &.--- & .0000848 & .0000228 & .00 & & .0000269 & .0000 & .00 \\
\hline & bbDDEe-- & ---- & .0001183 & .0000355 & .0000050 & & .0000848 & .0000228 & .0000030 & & .0000269 & .0000057 & .0000006 \\
\hline & bbDdEE -- & $-\ldots$ & .0001183 & .0000355 & .0000050 & ---- & .0000848 & .0000228 & .0000030 & & .0000269 & .0000057 & .0000006 \\
\hline a & BBDDEE - - & ---- & .0001183 & .0000057 & .0000002 & & .0000763 & .0000032 & .0000001 & & .0000188 & .0000005 & .0000000 \\
\hline A & BbDdEe--. & 800000 & .0105917 & .0011436 & .0001101 & 0694444 & .0079346 & .0007837 & .0000709 & .0413223 & .0032196 & .0002544 & .0000197 \\
\hline & BBDDEe -- & -... & .0001775 & .0000193 & .0000012 & & .0001187 & .0000115 & .0000007 & & .0000322 & .0000024 & .0000001 \\
\hline & BBDdEE - - & -... & .0001775 & .0000193 & .0000012 & -...- & .0001187 & .0000115 & .00 & ..... & .0000322 & .000 & \\
\hline & BbDDEE -- & $-\cdots$ & .0001775 & .0000193 & .0000012 & & .0001187 & .0000115 & .0000007 & & .0000322 & .0000024 & .0000 \\
\hline & BBddee -- & & .0000592 & .0000361 & .0000102 & & .0000424 & .0000246 & .00 & & .0000134 & .0000070 & .000 \\
\hline & bbDDee -- & & .0000592 & .0000361 & .0000 & $-\cdots$ & .0000424 & .0000246 & .00 & --.-. & .0000134 & .000 & \\
\hline & bbddEE -- & & .0000592 & .0000361 & .0000102 & & .0000424 & .0000246 & .0000066 & & .0000134 & .0000070 & .0000017 \\
\hline & Bb Ddee -- & --- & .0035503 & .0012517 & .0002725 & - & .0030518 & .0009578 & .000 & - & .0017737 & .0004201 & .0000729 \\
\hline & BbddEe -- & -- & .0035503 & .001 & .000 & & .0030518 & .0009 & .00 & -- & .0017737 & .000 & .00 \\
\hline & bbDdEe -- & $-\ldots$ & .0035503 & .0012517 & .0002725 & & .0030518 & .0009578 & .0001952 & & .0017737 & .0004201 & .0000729 \\
\hline & BBDDee -- & & 5592 & .0000161 & .0000022 & & .0000424 & .0000105 & .00 & & .0000134 & .0000027 & .0000003 \\
\hline & BBddEE -- & ----- & .0000592 & .0000161 & .000 & & .0000424 & .0000105 & .00 & & .0000134 & .000 & .00 \\
\hline & BDDDEE -- & $-\ldots-$ & .0000592 & .0000161 & .0000022 & & .0000424 & .0000105 & .0000013 & & .0000134 & .0000027 & .0000003 \\
\hline a & BBDdEe -- & -...- & .0002959 & .000 & .000 & & .00 & .0000 & & $\cdots$ & .0000591 & .0000118 & .0000013 \\
\hline & BbDDEe -- & $\ldots$ & .0002959 & .0000776 & .000 & & .0002035 & .0000492 & .000 & & .0000591 & .0000118 & .000 \\
\hline & BbDdEE -- & $-\ldots$ & .0002959 & .0000776 & .0000103 & $\ldots$ & .0002035 & .0000492 & .0000061 & ---- & .0000591 & .0000118 & .0000013 \\
\hline & Bbddee -- & ----- & .003 & & & & .00 & & & 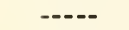 & & & \\
\hline & bbDdee -- & $\ldots-$ & .0034320 & .0022060 & .000 & - & .0029670 & .0018 & .00 & - & .0017468 & .0009 & .00 \\
\hline & bbddEe .- & -...- & .0034320 & .0022060 & .0009414 & $-\cdots$ & .0029670 & .0018160 & .0007391 & ---- & .0017468 & .0009584 & .0003495 \\
\hline a & BBDdee -- & $-\cdots$ & .000 & & & $-\cdots$ & & & & & & & \\
\hline & BBd & $-\cdots$ & .0001183 & .000 & & ---- & .000 & .000 & & --.- & 0269 & .000 & \\
\hline & BbDDee -- & n...- & .0001183 & .000 & .00 & -...- & .000 & .00 & & & 00269 & .000 & \\
\hline & BbddEE -- & $-\cdots$ & .0001183 & & & $-\ldots$ & & & & --- & & & \\
\hline & bЬDDEe -- & $-\cdots$ & .0001183 & .0000755 & .000 & $-\cdots$ & .000 & .000 & & & 0269 & & \\
\hline a & bЬDdEE -- & & .0001183 & .0000755 & .0000210 & & .0000848 & .0000512 & .0000135 & & .0000269 & .0000144 & .0000034 \\
\hline a & BbDdEe - - & 800000 & .0171006 & .0034913 & .0006344 & .0694444 & .0136312 & .0026006 & .0004491 & .0413223 & .0066488 & .0010709 & .0001630 \\
\hline a & BBddee -- & $-\cdots$ & .0000 & .000 & & $-\cdots$ & .000 & & & --.. & & & \\
\hline & $\mathrm{bbDDe}$ & -..- & .0000592 & .0000 & .000 & & .0000424 & .000 & & & 00134 & .000 & 0098 \\
\hline & bbddEE -- & -...- & .0000592 & .0000885 & .0000500 & ---- & .0000424 & .0000625 & .0000342 & $-\cdots$ & .0000134 & .0000193 & .0000098 \\
\hline & bbddee -- & $\cdots-$ & .0033728 & .0051970 & .0043725 & ---- & .0029246 & .0044527 & .0036610 & --- & .0017334 & .0025741 & .0020145 \\
\hline & BbDdee -. & ---- & & & & $-\cdots$ & & & & -..- & & & \\
\hline & BbddEe -- & -..-- & .0101775 & .0055 & .0021 & --- & .0088162 & & & $\cdots$ & .0052137 & .002 & .000 \\
\hline & bDDdEe -- & ---- & .0101775 & .0055125 & .0021133 & $\ldots-$ & .0088162 & .0044873 & .0016464 & $\ldots-$ & .0052137 & .0023081 & .0007653 \\
\hline & Bbddee -- & ----- & .010 & & & ---- & .00 & & & ---- & & & \\
\hline & bbDdee -- & & .0100592 & & & & .0087314 & & & & .0051868 & & .0043588 \\
\hline & bbddEe -- & --- & .0100592 & .0124229 & .0095862 & -... & .0087314 & .0105963 & .0079926 & $\ldots-$ & .0051868 & .0060680 & .0043588 \\
\hline & bbddee --. & 00000 & .3811238 & .4243955 & .4557521 & .3472222 & .4006701 & .4375470 & .4638448 & .4132231 & .4454857 & .4665629 & .4810991 \\
\hline
\end{tabular}


-able +0.--Relarive frequencies of female genotypes when native population ( 3 autosomal genes BBDDEE plus 1 sex-Iinked gene AA; female is neterogazetic) is overflooded bv $x$ release insects (abbddee, a-bbddee) for every native insect-continued

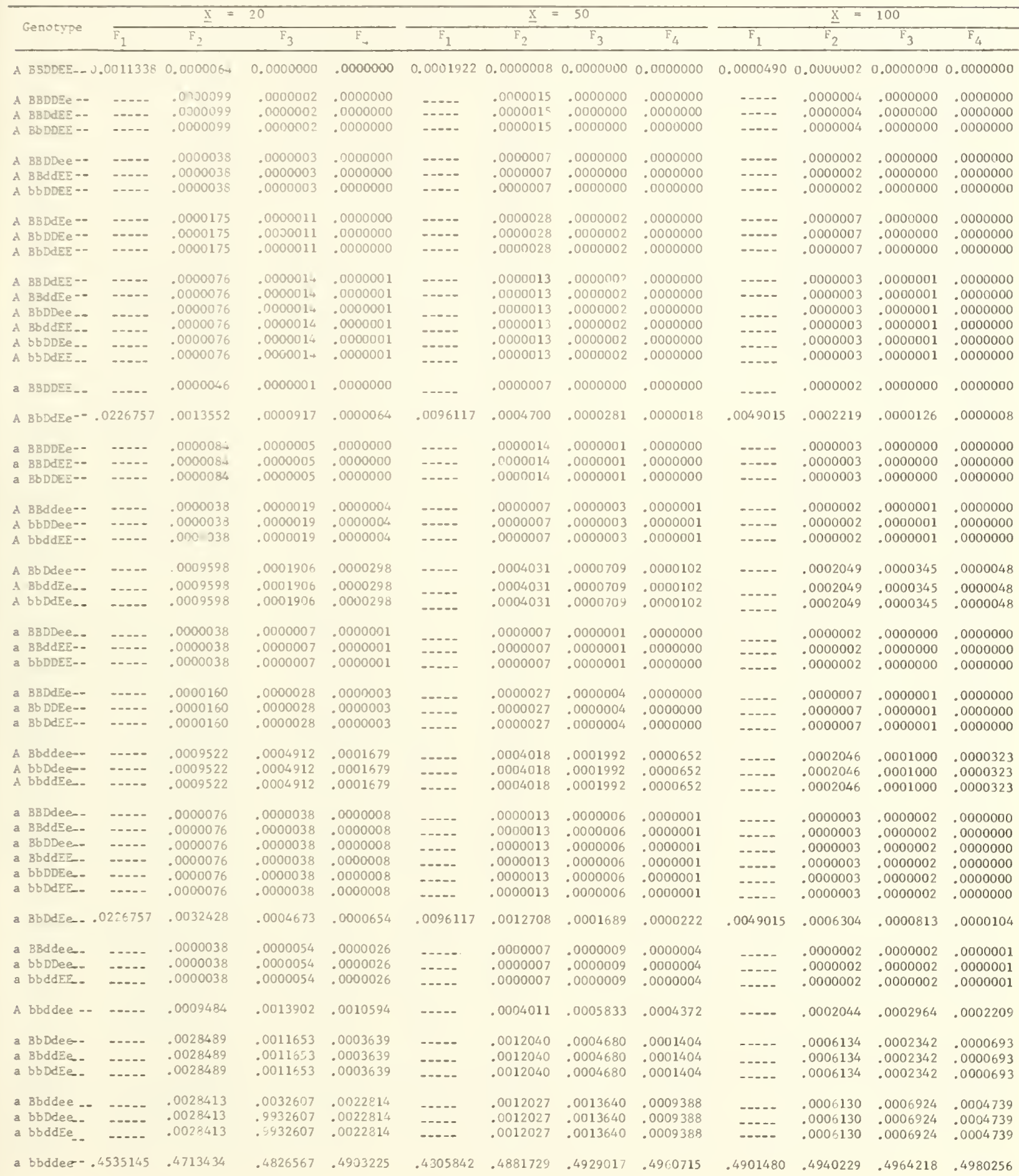


Table 40.--Relative frequencies of female genotypes when native population ( 3 autosomal genes BBDDEE plus 1 sex-1inked gene $\mathrm{AA}$; female is heterogametic) is overflooded by $\underline{X}$ release insects (aabbddee, a-bbddee) for every native insect--
Continued

\begin{tabular}{|c|c|c|c|c|c|c|c|c|c|c|c|}
\hline \multirow{2}{*}{ Genotype } & \multicolumn{4}{|c|}{$\underline{X}=200$} & \multicolumn{4}{|c|}{$\underline{x}=500$} & \multicolumn{3}{|c|}{$\underline{x}=1,000$} \\
\hline & $F_{1}$ & $\mathrm{~F}_{2}$ & $\mathrm{~F}_{3}$ & $F_{4}$ & $F_{1}$ & $\mathrm{~F}_{2}$ & $\overline{F_{3}}$ & $F_{4}$ & $\mathrm{~F}_{2}$ & $\mathrm{~F}_{3}$ & $\mathrm{~F}_{4}$ \\
\hline
\end{tabular}

A BBDDEE_. $0.00001240 .0000000 \quad 0.00000000 .0000000 \quad 0.0000020 \quad 0.00000000 .0000000 \quad 0.0000000$

\section{A
A
A
A
A
A}

BBDDEe--

(-.-$.0000000 \quad .0000000 \quad .0000000$

-... $\quad .0000000 \quad .0000000 .0000000$

A BBDDee-- -.-- .0000000 .0000000 .0000000

A BBddFE- $\quad 0000000.0000000 .0000000$

A bbDDEE-- $\quad .-.-.0000000 \quad .0000000 .0000000$

A BBDdEe-.

A $B$ DDDE

A BbDdEE--

A BBDdEE--

A BBddEe--

A BbDDee...

A BbddEE--

A bbDDEe.-

A bbDdEE--

a BBDDEE.-

A BbDdEe..-

a BBDDEe--

a BBDdEE--

a BbDDEE

A BBddee.-

A bbDDee

A bbddEE.-

A BbDdee

A Bbode

A bbDdEe..

a BBDDee--

a BBddEE--

a bBDDEE--

a BBDdEe $\rightarrow-$

a BbDDEe--

a BbDdEE -

A Bbddee--

A bbDdee--

A bbddEe --

a BBDdee -

a BBddEe --

a BbDDee - -

a BbddEE --

a bbDDEe --

a bbDdEE - -

a BBddee -

a bbDDee - - - -

a bbddEE - -

A bbddee... .....-

a BbDdee - -

a BbddEe - - .-.-

a bbDdEe - -

a Bbddee - . . ...-

a bbDdee ... ......

a bbddEe -.. ....-

$.0000002 \quad .0000000 .0000000$ .0000002 .0000000 .0000000 .0000002 .0000000 .0000000

.0000001 .0000000 .0000000 .0000001 .0000000 .0000000 .0000001 .0000000 .0000000 .0000001 .0000000 .0000000 .0000001 .0000000 .0000000 .0000001 .0000000 .0000000

$.0000000 \quad .0000000 .0000000$

.0001076

.0000001 .0000000 .0000000 .0000001 .0000000 .0000000 .0000001 .0000000 .0000000 .0000000 .0000000 .0000000 .0000000 .0000000 .0000000

.0001033 .0000170 .0000023 .0001033 .0000170 .0000023 $.0001033 \quad .0000170 .0000023$

.0000000 .0000000 .0000000 .0000000 .0000000 .0000000 .0000000 .0000000 .0000000

$.0000002 \quad .0000000 .0000000$ .0000002 .0000000 .0000000 .0000002 .0000000 .0000000

.0001032 .0000501 .0000160 .0001032 .0000501 .0000160 .0001032 .0000501 .0000160

.0000001 .0000000 .0000000 $.0000001 \quad .0000000 .0000000$ .0000001 .0000000 .0000000 .0000001 .0000000 .0000000 .0000001 .0000000 .0000000 .0000001 .0000000 .0000000

.0003139

$.0000000 \quad .0000000 .0000000$

.0000000 .0000000 .0000000

.0000000 .0000000 .0000000

$.0001032 \quad .0001494 .0001111$

$.0003096 \quad .0001171 .0000344$

$.0003096 \quad .0001171 .0000344$

$.0003096 \quad .0001171 .0000344$

$.0003095 \quad .0003489 .0002381$

$.0003095 \quad .0003489 \quad .0002381$
$.0000000 \quad .0000000 .0000000$

$\begin{array}{llll} & .0000000 \quad .0000000 \quad .0000000\end{array}$ $\left[\begin{array}{llll}.0 & .0000000 \quad .0000000 \quad .0000000\end{array}\right.$ $\begin{array}{llll}0.0 & .0000000 & .00000000 & .0000000\end{array}$

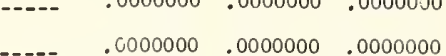
$\begin{array}{llll}-.0- & .0000000 & .0000000 & .0000000\end{array}$ $\begin{array}{llll}{[. .-} & .0000000 & .0000000 & .0000000\end{array}$

$\begin{array}{lllll}0.0000000 & .0000000 & .0000000\end{array}$

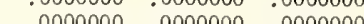
$\begin{array}{llll}{[.-2} & .0000000 & .0000000 & .0000000 \\ \ldots . . & .0000000 & .0000000 & .0000000\end{array}$

. . .0000000 .0000000 .0000000

[.- $\quad .0000000 \quad .0000000 \quad .0000000$

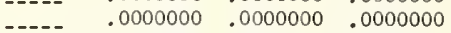

.0000000 .0000000 .0000000

[.. $\quad .0000000 \quad .0000000 \quad .0000000$

$\begin{array}{llll}0.0000000 & .0000000 & .0000000\end{array}$

$\begin{array}{cccc}0.0 & .0000000 \quad .0000000 \quad .0000000\end{array}$

$\begin{array}{llll}0009960 & .0000422 & .0000023 & .0000001\end{array}$

[-.. $\quad .0000000 \quad .0000000 \quad .0000000$

[... $\quad .0000000 \quad .0000000 \quad .0000000$

$\begin{array}{llll}0.0000000 & .0000000 & .0000000\end{array}$

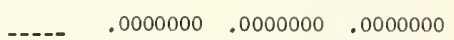

.0000000 .0000000 .0000000

.0000000 $.0000000 \quad .0000000$

$\begin{array}{llllll} & .0000415 & .0000067 & .0000009\end{array}$

$\begin{array}{llll}--1.0000415 & .0000067 & .0000009\end{array}$

\begin{tabular}{llll}
{$[-0$.} & .0000415 & .0000067 & .0000009 \\
\hdashline-0 & .0000415 & .0000067 & .0000009
\end{tabular}

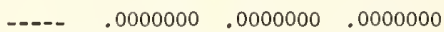

$\begin{array}{llll}.0000000 & .0000000 & .0000000\end{array}$

$\begin{array}{llll}--.- & .0000000 & .0000000 & .0000000\end{array}$

$0.0000000 \quad .0000000 \quad .0000000$

$\begin{array}{llll}0.00 & .0000000 & .0000000 & .0000000\end{array}$

... $\quad .0000000 \quad .0000000 \quad .0000000$

$\begin{array}{llllll}0.0000415 & .0000201 & .0000064\end{array}$

[... $\quad .0000415 \quad .0000201 \quad .0000064$

... $\quad .0000415 \quad .0000201 \quad .0000064$

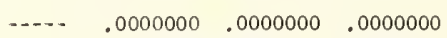

$\left[\begin{array}{llll}.00 & .0000000 & .0000000 \quad .0000000\end{array}\right.$

$\begin{array}{llll}.+- & .0000000 & .0000000 & .0000000\end{array}$

n. $\quad .0000000 \quad .0000000 \quad .0000000$

[.. $\quad .0000000 \quad .0000000 \quad .0000000$

-.- $\quad .0000000 \quad .0000000 \quad .0000000$

.0000399 .0000050 .0009960

$.0001252 \quad .0000158 \quad .0000020$

---

$.0000000 \quad .0000000 \quad .0000000$

$.0000000 \quad .0000000 \quad .0000000$

$.0000000 \quad .0000000 \quad .0000000$$$
\begin{array}{llll}
0.000415 & .0000601 & .0000446
\end{array}
$$

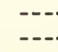

-.- . .0001245 .0000469 .0000137

$\begin{array}{llll}.0001245 & .0000469 \quad .0000137\end{array}$

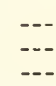

0.00000050 .00000000 .00000000 .0000000

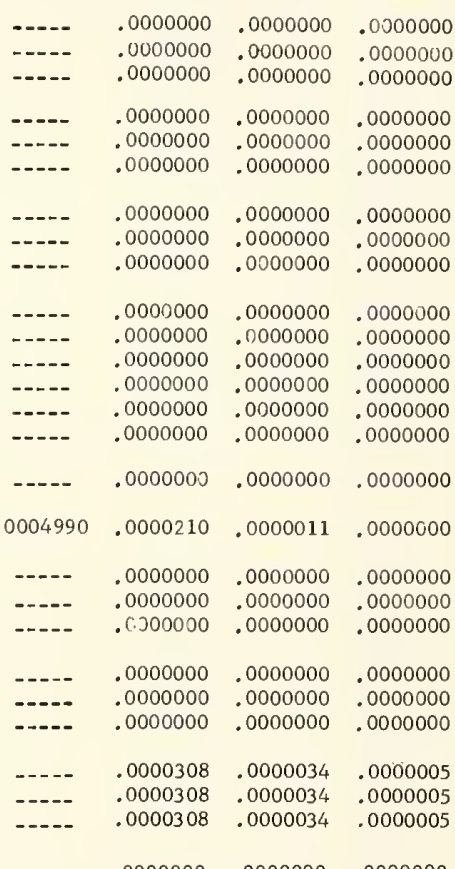

[.- $\quad .0000000 \quad .0000000 \quad .0000000$

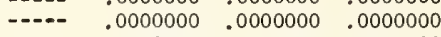

..- $\quad .0000000 \quad .0000000 .0000000$

-.- $.0000000 \quad .0000000 \quad .0000000$

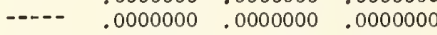

... .0000000 .0000000 .0000000

[.. $\quad .0000208 \quad .0000100 \quad .0000032$

$\left[\begin{array}{llll}0 .-0 & .0000208 & .0000100 \quad .0000032\end{array}\right.$

$\begin{array}{ccc}0 .- & .0000208 \quad .0000100 \quad .0000032\end{array}$

.... . .0000000 .0000000 .0000000

-.- $.0000000 \quad .0000000 .0000000$

$\begin{array}{llll}.0000000 & .0000000 \quad .0000000\end{array}$

--- $\quad .0000000 \quad .0000000 \quad .0000000$

... .0000000 .0000000 .0000000

... $.0000000 \quad .0000000 \quad .0000000$

$\begin{array}{llll}.0004990 & .0000626 \quad .0000078 & .0000010\end{array}$

-. $\quad .0000000 \quad .0000000 \quad .0000000$

[-. $\quad .0000000 \quad 0000000.0000000$

..- $.0000000 \quad .0000000 \quad .0000000$

[-.. $\quad .0000208 \quad .0000301 \quad .0000223$

$\begin{array}{llll}0.0000624 & .0000234 \quad .0000068\end{array}$

$\begin{array}{llll}-.-0 & .0000624 & .00000234 & .00000068\end{array}$

[.. $\quad .0000624 \quad .0000234 \quad .0000068$

$\begin{array}{llll}0.0000624 & .0000702 & .0000478\end{array}$

... $.0000624 \quad .0000702 \quad .0000478$

$\begin{array}{llll}0 .- & .0000624 & .0000702 & .0000478\end{array}$

a bbddee $=.4950372 \quad .4969961 \quad .4982044 .4990106 \quad 4980059 \quad .4987939 \quad .4992795 \quad .4996032$ 
iable ---Suppression of native population 10,000 insects, $1: 1$ sex ratto) by overflooding generations with release straln bearing conditionsl lechal traits when population is held static by conventional means

\begin{tabular}{|c|c|c|c|c|c|c|c|c|c|c|c|}
\hline \multirow[t]{2}{*}{ Case } & \multicolumn{2}{|c|}{ Genotrres of-- } & \multicolumn{3}{|c|}{$\begin{array}{c}\text { Inheritance of conditional lethal traits } \\
\text { in release strain }\end{array}$} & \multirow{2}{*}{$\begin{array}{l}\text { Release, } \\
\text { ratial' }\end{array}$} & \multirow[t]{2}{*}{ Sex } & \multicolumn{4}{|c|}{ Number of viable genotypes in-- } \\
\hline & $\begin{array}{l}\text { Soctue } \\
\text { serain }\end{array}$ & $\begin{array}{l}\text { Release } \\
\text { scrain }\end{array}$ & Trait 1 & Trait 2 & Trait 3 & & & $F_{1}$ & $E_{2}$ & $\mathrm{~F}_{3}$ & $\mathrm{~F}_{4}$ \\
\hline \multicolumn{12}{|c|}{ Oerflooded in parental generation } \\
\hline $1 \mathrm{a} \ldots$ & as & A & 1 doe, auto. &.-- & $\ldots$ & so & - & 4 & 4 & 4 & 4 \\
\hline $16 \ldots$ & as & A & 1 doe.auto. & $\cdots$ & $\ldots$ & 100 & - & 1 & 1 & 1 & 1 \\
\hline $2 a \ldots$ & AABB & sabb & 2 auco. $(3, \dot{i})$ & $\cdots$ & $\ldots$ & 5D & - & 4 & 5 & 3 & 2 \\
\hline $2 b=--$ & AN3 & Aabb & 2 euto. $(3, \infty)$ &.-- &.-- & $10 D$ & - & 1 & 1 & 1 & 1 \\
\hline $3 \cdots$ & ASB & aabb & 2 auto. $(2-\infty)$ & .... & $\infty$ & 1,000 & - & 20 & 10 & 5 & 3 \\
\hline$\ldots$ & AABBDD & Aabbdd & 3 auto. $(4-6)$ & $\ldots$ & $\cdots$ & 100 & - & 1 & 1 & 1 & 0 \\
\hline $52 \ldots$ & AHSBDOEE & aabbddee & H suto. $(6-8)$ & $-\ldots$ & $\ldots$ & 20 & - & 23 & 12 & 3 & 1 \\
\hline $5 b=\ldots$ & AABEDDEE & abbbddee & $\therefore$ auto. $0-8)$ & $\ldots$ & $\ldots$ & 50 & - & 4 & 2 & 0 & 0 \\
\hline $6=\ldots$ & AABBDDEE & abbddee & 3 euto.t 1 s.1. $(6-9)$ & $\cdots$ & $-\cdots$ & so & M & 194 & 31 & 7 & 2 \\
\hline $6 a-$. & AMBDDEE & aabbddee & 3 auto. +1 s.1. $(6-9)$ & $\cdots$ & --- & so & $\mathrm{F}$ & 98 & 16 & 3 & 1 \\
\hline$o b=.-$ & AMBB JDEE & aabbddee & 3 auto. - I s.1. (6-9) & $\ldots .$. & $-\ldots$ & 200 & M & 50 & 8 & 1 & 0 \\
\hline $6 b=.-$ & AABBDDEE & aubbddee & 3 auto.t I s.1. $(6-9)$ & $-\ldots$ & $\ldots$ & 200 & $\mathrm{~F}$ & 25 & 3 & 1 & 0 \\
\hline $7 \ldots$ & $a a b b$ & MBS & 1 dow auto. & I dos.auto. & $\cdots$ & 50 & - & 4 & 1 & 0 & 0 \\
\hline$\hat{s}+\ldots$ & LaBBDo & Aubbodd & I dos.auto. & 2 auco. $(3,4)$ & $\ldots$ & 50 & - & 4 & i & 0 & 0 \\
\hline शेa & AABBDDEE & Anbldee & 1 doo.auto. & 3 suto. $(--6)$ & --- & $2 D$ & - & 23 & 9 & 2 & 0 \\
\hline $9 \mathrm{~b}---$ & A.SBDDEE & Anbbddee & I doesauto. & 3 suto, $(6-6)$ & $\ldots$ & 50 & - & 4 & 1 & 0 & 0 \\
\hline 10 en. & MBBDDEE & aabbddec & 2 suto. $(3,6)$ & 2 auto. $(3,4)$ & -..- & $2 D$ & - & 23 & 10 & 2 & 0 \\
\hline $108=-$ & MABBDDEE & Aabbidee & 2 auto. $(3, \infty)$ & 2 auto. $(3,4)$ & $\ldots$ & so & - & 4 & 1 & D & 0 \\
\hline$\therefore: \ldots$ & AABBDOEE & Aabbddee & 1 duc.s.1. & 3 suto. $(k-6)$ & $\ldots$ & 10 & M & 41 & 29 & 8 & 2 \\
\hline $11=--$ & AABBDDEE & Anboddee & 1 doo.s.1. & 3 auto. $(4-6)$ & $\ldots$ & 10 & $F$ & 41 & 33 & 15 & 5 \\
\hline $113=$ & $\triangle A B B D D E E$ & Aubbddee & 1 doo.s.1. & 3 auto. $(4-6)$ & $-\cdots$ & so & M & 2 & 1 & 0 & 0 \\
\hline $11 \mathrm{~b}=-$ & A $\triangle B B D D E Z$ & Ansbddee & 1 dow.5.1. & 3 auco. $(4-6)$ & $-\ldots$ & 50 & $\mathrm{~F}$ & 2 & 1 & 0 & 0 \\
\hline $12 a$ & sabbdd & AABDD & I does.auco. & 1 dom.euto. & 1 doch.auto. & 10 & - & 83 & 8 & 1 & D \\
\hline $12 \mathrm{~b}=-$ & aabbdd & $A A B D D$ & 1 doo. suto. & I dow.suto. & 1 dow.suto. & so & - & 4 & 0 & 0 & 0 \\
\hline $13 \mathrm{a--}$ & aAb DDDE: & MABsddee & 1 dor, auto. & I don, auto. & 2 auto. $(3,4)$ & 10 & - & 83 & 11 & 1 & D \\
\hline $138=-$ & aSSDDEE & AABBddee & 1 don.auto. & I dotanto. & 2 auto. $(3,4)$ & 20 & - & 23 & 2 & D & 0 \\
\hline $1-a=$ & abbDDEE & AABBddee & 1 dor.tuto. & 1 dow.auto. & 2 auto. $(2-4)$ & 10 & - & 83 & 19 & 4 & 1 \\
\hline $1-3=$ & 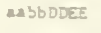 & AABBddee & I doc.auco. & I dow.auco. & 2 auto. $(2-4)$ & 50 & - & 4 & 1 & 0 & 0 \\
\hline $15 a--$ & AABDDDEE & AABBddee & 1 doo.s.1. & I doen, auto. & 2 auto. $(3,4)$ & 20 & M & 11 & 1 & 0 & 0 \\
\hline $15 a--$ & aAbDDDEE & MBsddee & 1 doo.s.1. & I doe auto. & 2 auto. $(3,4)$ & 20 & F & 11 & 2 & 0 & 0 \\
\hline $15 b=-$ & aAb bDOEE & AABBddee & 1 dom.s.1. & 1 doe.auto. & 2 auto. $(3,4)$ & 50 & M & 2 & 0 & 0 & 0 \\
\hline $158=-$ & AABb DOEE & MABBddee & 1 doo.s.1. & 1 dom.auto. & 2 suto. $(3,4)$ & 50 & $F$ & 2 & 0 & 0 & 0 \\
\hline
\end{tabular}

Jverflooded in parental, $F_{1}, F_{2}$, and $F_{3}$ generations

\begin{tabular}{|c|c|c|c|c|c|c|c|c|c|c|}
\hline $16 a--a$ & AA & I don,auto. & $\cdots$ & $\cdots$ & 10 & - & 83 & 10 & 2 & D \\
\hline $16 \mathrm{~b}-$. & An & 1 dom.ante. & 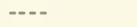 & $\cdots$ & 50 & - & 4 & 0 & 0 & 0 \\
\hline 17 A $\ldots$ AABB & aabb & 2 suto. $(3,4)$ & $\cdots$ & $-\cdots$ & 5 & - & 278 & 51 & 8 & 1 \\
\hline $175--$ MBB & aabb & 2 suto. $(3,4)$ & $\cdots$ & $\cdots$ & so & - & 6 & 1 & 0 & 0 \\
\hline :BA $\ldots$ MBB & aabb & 2 suto. $(2-4)$ & $\ldots$ & $-\cdots$ & 100 & - & 197 & 34 & 7 & 2 \\
\hline $18 \mathrm{~b}=\mathrm{AABB}$ & aabb & 2 auto. $(2-4)$ & $\cdots$ & $\cdots$ & 1,000 & - & 20 & 3 & 1 & 0 \\
\hline $19 \mathrm{a}--\mathrm{MBBDD}$ & mabbdd & 3 suto. $(4+6)$ & $\ldots$ & $\ldots$ & 10 & - & 83 & 14 & 2 & 0 \\
\hline $19 \mathrm{~b}--\mathrm{AABBDD}$ & abbdd & 3 auto. $(4-6)$ & $\cdots$ & $--\cdot-$ & so & - & 4 & 1 & 0 & 0 \\
\hline $20 \mathrm{~A}-$. AABBDDEE & aabbddee & 4 auto. $(6-8)$ & $\cdots$ & -... & 5 & - & 278 & 32 & 2 & 0 \\
\hline 206-- AMBBDIE & aubbddee & Cs auto. $(6-8)$ & $\cdots$ &..- & 20 & - & 23 & 2 & 0 & 0 \\
\hline 21A-- ABBBDDEE & abbddee & 3 auto. + 1 s.1. (6-9) & .... & $-\cdots$ & 4 & M & 1,850 & 248 & 26 & 2 \\
\hline $21 \mathrm{AM}-\mathrm{AABBDDEZ}$ & asbbdidee & 3 auto. +1 s.1. (6.9) & .... & -..- & 4 & E & 1,000 & 147 & 16 & 2 \\
\hline 216 .. MABBDDEE & a.jbbddee & 3 auto. +1 s.1. $(6-9)$ & $\cdots$ & --- & 200 & M & so & 2 & 0 & 0 \\
\hline $21 \mathrm{~b} \ldots-$ ABBDDEE & aabbddee & 3 auto. +1 s.1. $(6-9)$ & $-\ldots$ & $\cdots$ & 200 & g & 25 & 1 & 0 & 0 \\
\hline $22 a--a b b$ & $A \mathrm{ABB}$ & 1 dom,auto. & 1 dom.auto. & ---- & 3 & - & 625 & 39 & 3 & 0 \\
\hline $22 b \ldots a b b$ & MABB & I does.auto. & I doen.auto. & --- & 10 & - & 83 & 3 & 0 & 0 \\
\hline $23 \mathrm{a}--$ aaBBDD & Mbbdd & 1 doe, auto. & 2 auto. $(3,4)$ & -..- & 3 & - & 625 & 52 & 2 & 1 \\
\hline $23 b \ldots a$ a BBDD & Aabbdd & I doceauto. & 2 auco. $(3,4)$ & $\ldots$ & 10 & - & 83 & 4 & 2 & 0 \\
\hline 24a-- aABBDDEE & Aabbddee & 1 dom suto. & 3 auto. $(4-6)$ & $\ldots$ & 3 & - & 625 & 58 & 3 & 0 \\
\hline 24b-- AaBBDDEE & Mabbidee & I dortauto. & 3 auto. $(4-6)$ & $-\ldots$ & 20 & - & 23 & 1 & 0 & 0 \\
\hline 25a - - MABB DDEE & a abbddee & 2 auto. $(3,4)$ & 2 auto. $(3,4)$ & -... & 3 & - & 625 & 70 & 4 & 1 \\
\hline $25 \mathrm{~b}-$. AMBBDDEE & aabbddee & 2 auto. $(3,4)$ & 2 auto. $(3,4)$ & $\ldots$ & 20 & - & 23 & 1 & 0 & 0 \\
\hline $25 a--$ a & AAbbddee & 1 dom. 3.1 . & 3 auto. (4.6) & $\ldots$ & 2 & M & 556 & 67 & 5 & 1 \\
\hline $25 \mathrm{a}-$. aBBDDEE & Mabbidee & $1 \mathrm{dom} \cdot 3.1$. & 3 auto. $(4-6)$ & $-\ldots$ & 2 & 8 & 556 & 65 & 11 & 1 \\
\hline $26 \mathrm{~b} \ldots \mathrm{a} a \mathrm{BBDDEE}$ & Mabbddee & I doen.s.1. & 3 auto. $(4-6)$ & -..- & 20 & $M$ & 11 & 1 & 0 & 0 \\
\hline $26 b-$. aaBBDDEE & Aabbddee & l dom.3.1. & 3 auto. $(4-6)$ & -... & 20 & 8 & 11 & 1 & 0 & 0 \\
\hline $27 a=-$ aabbdd & AABBDD & I dom.auto. & 1 dom.auto. & 1 dom.auto. & 2 & - & 1,111 & 51 & 2 & 0 \\
\hline 27b-- aabbdd & AABBDD & 1 don.auto. & 1 dom.auto. & 1 dom.auto. & 10 & - & 83 & 1 & D & 0 \\
\hline $28 \mathrm{a}$.. aabbDDEE & AABBddee & 1 doe.auto. & 1 dom.auto. & 2 auto. $(3,4)$ & 2 & - & 1,111 & 64 & 2 & 1 \\
\hline $28 \mathrm{~b}$... aabbDDEE & AaBBAdee & 1 dow,auto. & I dom,auto. & 2 aut $0 .(3,4)$ & 10 & - & 83 & 1 & 0 & 0 \\
\hline $29 a--$ aabbDDEE & AABBCdee & 1 dom.auto. & I dom,auto. & 2 auto. $(2-4)$ & 3 & - & 625 & 35 & 2 & 1 \\
\hline $29 b--a a b b D D E E$ & ARBBddee & I dom,auto. & I dow.auto. & 2 auto. $(2-4)$ & 20 & - & 23 & 1 & 0 & 0 \\
\hline 30a-- aabbDDEE & AABBddee & $1 \mathrm{dam} \cdot 8.1$ & I dom.auto. & 2 auto. $(3,4)$ & 1 & M & 1,250 & 143 & 9 & 0 \\
\hline 30a... a abb DDEE & MABBddee & 1 dom.3.1. & 1 don.auto. & 2 auto. $(3,4)$ & 1 & 8 & 1,250 & 178 & 16 & 1 \\
\hline $30 \mathrm{Ob}$ - - aabb DDEE & AABBddee & 1 dom.s.1. & I dom.auto. & 2 auto. $(3,4)$ & 10 & M & 41 & 1 & 0 & 0 \\
\hline 308 -. aabb DDEE & AABBddee & $1 \mathrm{dos} .3 .1$. & $1 \mathrm{dcm}$,auto. & 2 auto. $(3,4)$ & 10 & $F$ & 41 & 1 & 0 & 0 \\
\hline
\end{tabular}

thomer of relesse Insects for every native insect. 
